Portland State University

PDXScholar

6-10-2019

\title{
Identifying with Remida: Early Childhood Educators' Experiences with Reuse Materials in Reggio Emilia Inspired Identity Studies
}

Michelle Angela Domingues

Portland State University

Follow this and additional works at: https://pdxscholar.library.pdx.edu/open_access_etds

Part of the Curriculum and Instruction Commons, and the Early Childhood Education Commons Let us know how access to this document benefits you.

Recommended Citation

Domingues, Michelle Angela, "Identifying with Remida: Early Childhood Educators' Experiences with Reuse Materials in Reggio Emilia Inspired Identity Studies" (2019). Dissertations and Theses. Paper 5492. https://doi.org/10.15760/etd.7366

This Dissertation is brought to you for free and open access. It has been accepted for inclusion in Dissertations and Theses by an authorized administrator of PDXScholar. Please contact us if we can make this document more accessible: pdxscholar@pdx.edu. 
Identifying with Remida: Early Childhood Educators' Experiences with Reuse Materials in Reggio Emilia Inspired Identity Studies

by

Michelle Angela Domingues

A dissertation submitted in partial fulfillment of the requirements for the degree of

Doctor of Education

in

Education Leadership: Curriculum and Instruction

Dissertation Committee:

Will Parnell, Chair

Christine Chaillé

Samuel Henry

Alma Trinidad

Portland State University

2019 
(C) 2019 Michelle Angela Domingues 


\begin{abstract}
Mainstream approaches to identity studies in early childhood education (ECE) are problematic from both social constructivist and early childhood education for sustainability perspectives. Mainstream approaches are influenced by behaviorist theories of developmental psychology literature, which privilege individual identity, whereas emerging discourses in identity theory trend toward discussing the multiplicity of social identities. Alternatively, and in contrast to traditional behaviorist and cognitive learning theories, cultural centers like Reggio Emilia, Italy’s Remida Creative Recycling Center promote reuse material projects that socially construct identities in Reggio's Municipal Infant/Toddler Centers and Preprimary Schools by giving value to waste materials through offering such materials in young children's work and thinking. For the purposes of this research study, I embarked on a quest to understand what early childhood educators' experiences of socially constructing identity studies with young children meant to them.
\end{abstract}

As a narrative inquirer, I conducted dyadic interviews and focus group discussion over the course of three two-hour sessions to illuminate six early childhood educator's experiences of socially constructing identity studies with young children as a Remidainspired educator. The first two dyadic narrative sessions document participants reuse collages, Remida-inspired documentation, and narrative data that highlight professional formation patterns, metaphors and unique points of view or 'Hidden Treasures' that emerged during the interviews. Throughout the three sessions, at the end of each inquiry, I use a re-storying method to summarize key points made by all the participants and to 
express multiple perspectives through a collective voice. As researcher, I employed Anzaldua's (1987) concept of "Borderlands" to develop an understanding of early childhood educator's experiences of socially constructing identity studies with young children as a Remida- inspired educator.

Although each participant's experiences were unique, patterns came into view which are interpreted through Katz (1972) framework for developmental stages of preschool teachers: survival, consolidation, renewal, and maturity. These growth patterns are further aligned with three corresponding archetypal stages of Remida-inspired identity studies that emerged from the collage and interview process, namely, (a) Identifying with Reggio Emilia philosophies: The Forager, (b) Identifying with Remida: The Global Homesteader and (c) Socially Constructing Identity Studies: The Navigator. Additionally, nine Hidden Treasures became visible from each stage including, Children's Identities are More Fluid, and Remida Materials Support Constructivist Identity Studies. These Hidden Treasures illuminate participants experiences and are synthesized with a Borderlands framework as affirmation of an image of the child as a global ecological citizen, capable of understanding and expressing more complex features of social identities. 


\section{Dedication}

I dedicate this work to Border crossers and to the image of the child as a global ecological citizen, capable of understanding and expressing more complex features of social identities. 


\section{Acknowledgements}

I hold in the highest esteem, my teachers from the academic worlds of education and Chicana/o studies, and would like to thank: My first Chicano teacher and mentor, Salvador Reza, who introduced me to the spiritual medicine of the temescal sweat lodge and first nurtured me as a bilingual educator, Dr. Roberto De Anda, whose mentorship guided me through a post-Bach and graduate Chicano/Latino studies and into predoctoral studies, Tony Martinez, who convinced me si se puede 'yes it can be done!' and who recommended me for graduate school, Dr. Pramod Parajuli, whose cosmovision awakened my consciousness and taught me how to weave together ecological insight and scholarship, Dr. Julia Curry Rodriguez, who introduced me to graduate level critical theory and the discipline of a Chicana/o studyist, Dr. Morra Torres, whose love for Mexican counter-history awoke the same in me, Dr. Perlita R. Dicochea, who taught me academic stamina, introduced me to Chicana/o environmental justice scholarship and encouraged me to pursue doctoral level work, Dr. Rosalyn McKeown, for acting as a guide between academic worlds, encouraging me to integrate Chicana/o theory into education for sustainable development, Dr. Andy Job, for facilitating a deeper awareness of myself as an adult learner while making me laugh, and Dr. Devon G. Pena, whose environmental justice scholarship and intellectual mentorship during my graduate training has assured me that ecology and Chicana/o studies are not only deeply connected, but are in fact "subversive kin." Finally, to Dr. Will Parnell, for passing the spark of Remida onto me and committing to come alongside me on this quest. 


\section{Table of Contents}

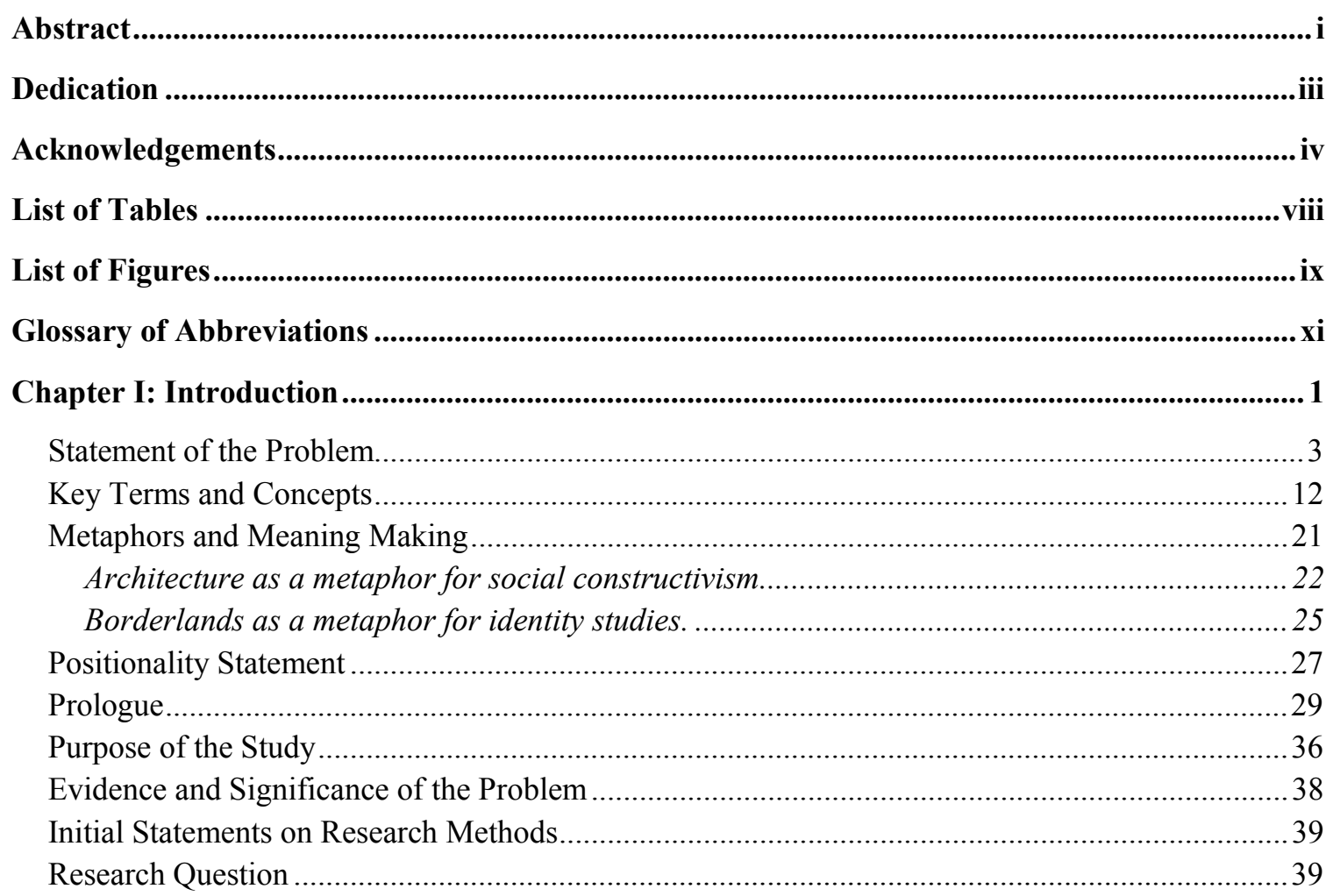

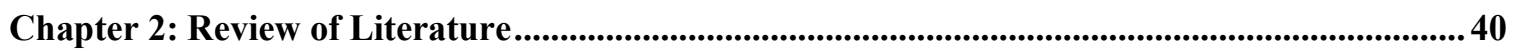

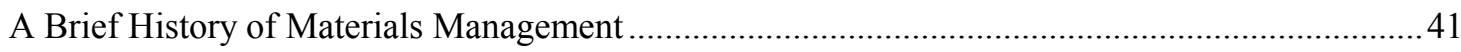

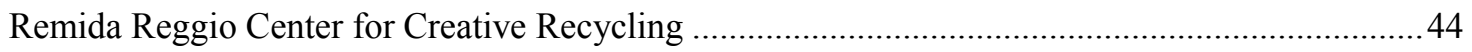

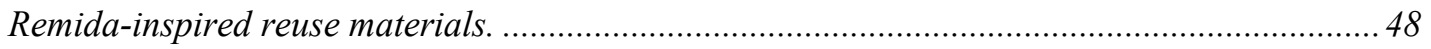

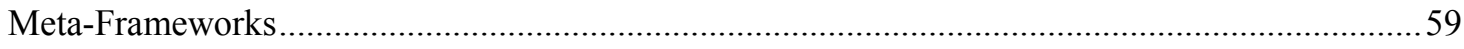

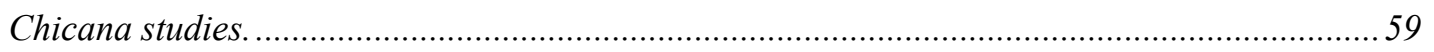

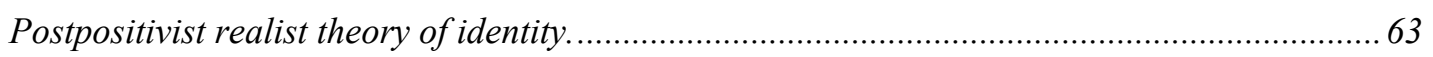

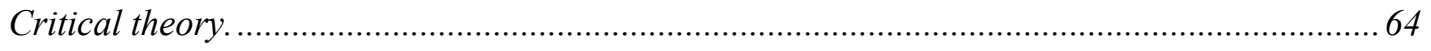

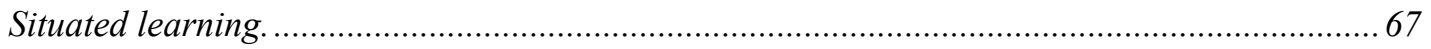

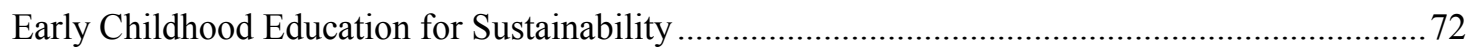

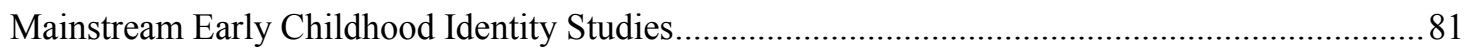

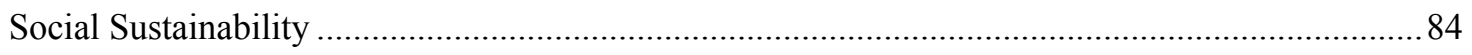

Social Constructivism as a Theoretical Basis of Early Childhood Education .................................91

Socially Constructing Identities in Early Childhood Education ....................................................95

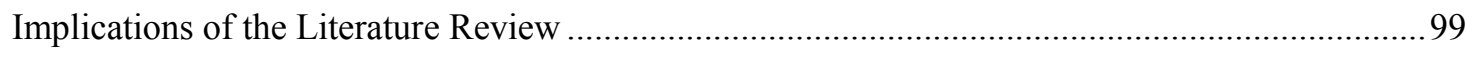

Chapter 3: Methods and Approaches..................................................................................................... 101

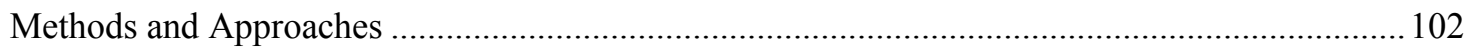

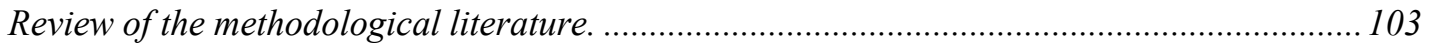

Narrative inquiry for investigating notions of identity formation ............................................ 108 


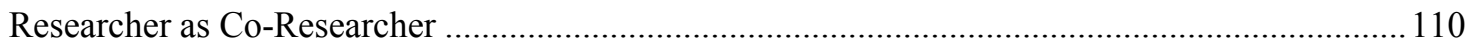

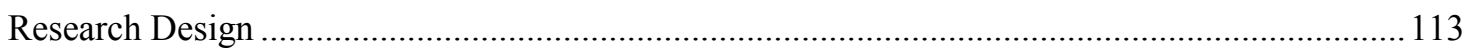

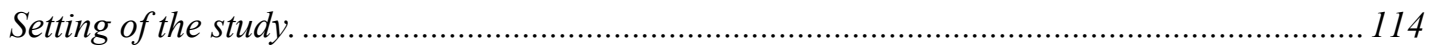

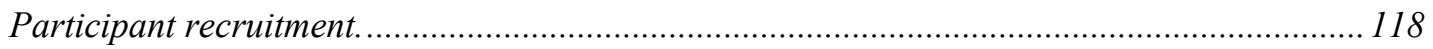

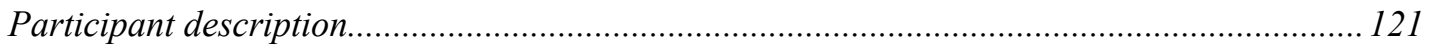

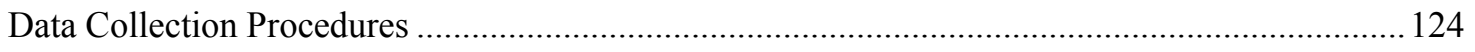

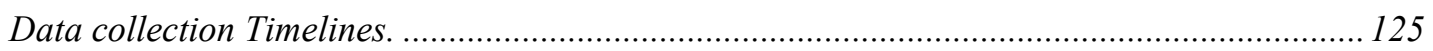

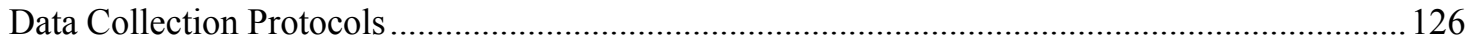

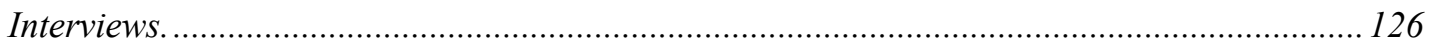

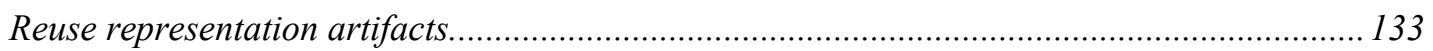

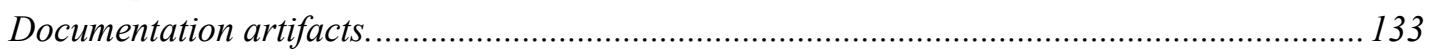

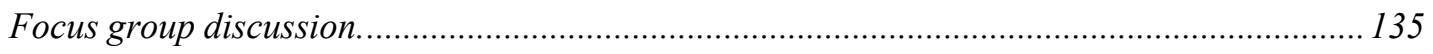

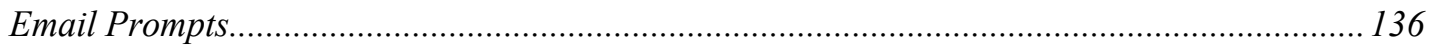

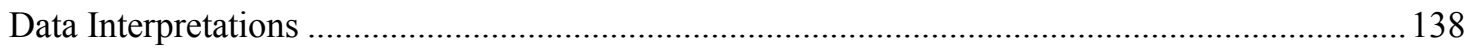

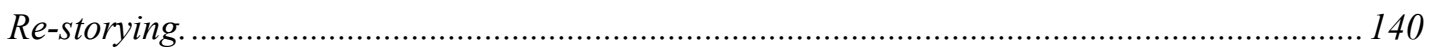

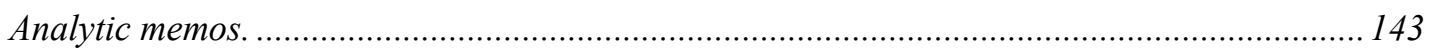

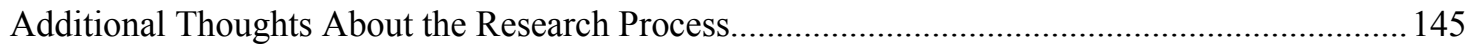

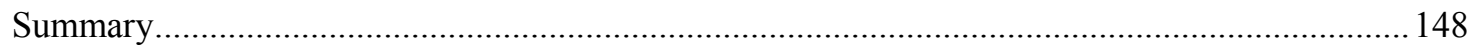

Chapter 4: Foragers, Homesteaders, Navigators .................................................................................... 150

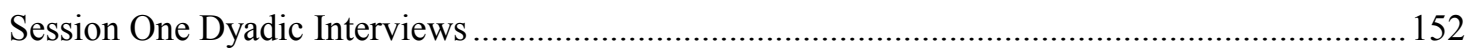

The first narrative inquiry prompt: The Forager................................................................. 153

Data interpretations one: Survival stage growth patterns - The Forager .............................. 165

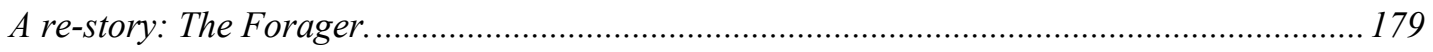

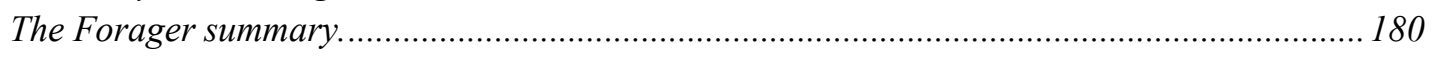

The second narrative inquiry prompt: The Global Homesteader. ............................................. 181

Data interpretations two: Consolidation and renewal stage growth patterns-The Global

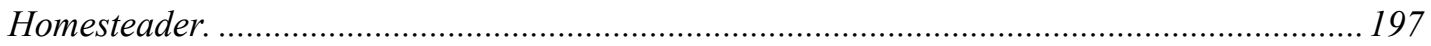

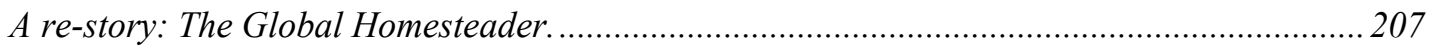

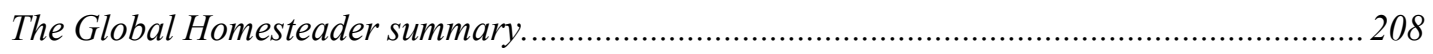

The third narrative inquiry prompt: The Navigator.............................................................. 211

Data interpretations three: Mature stage growth patterns - The Navigator. ………………..... 221

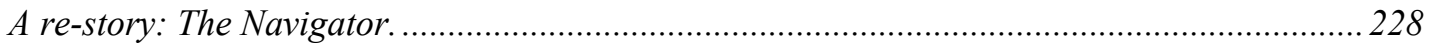

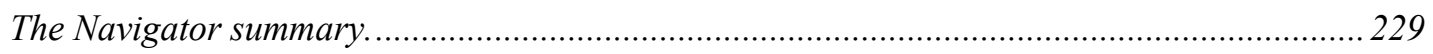

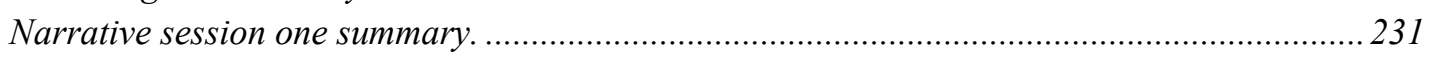

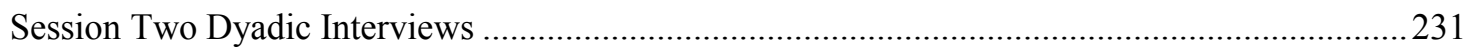

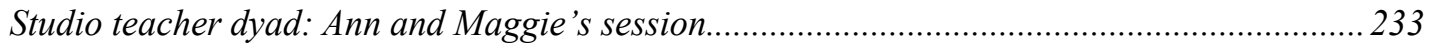

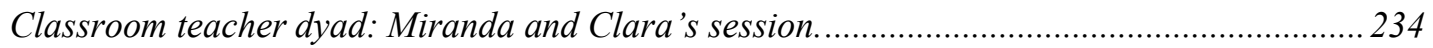

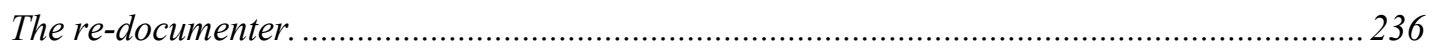

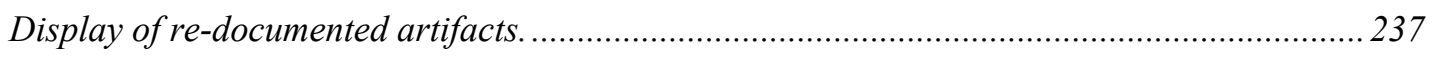

Data interpretations three: Growth pattern-The re-documenter.......................................... 263

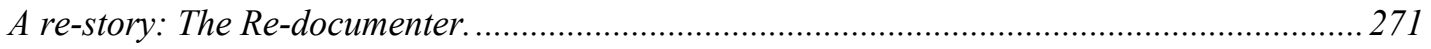

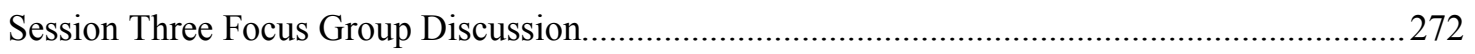


Focus group.

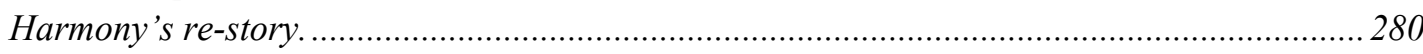

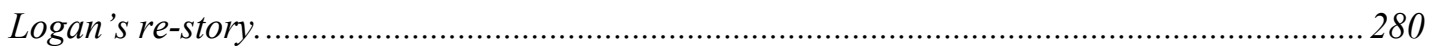

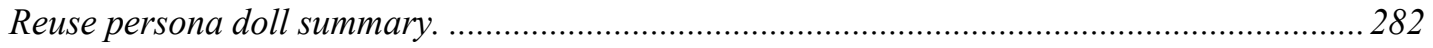

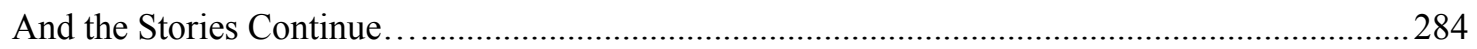

Chapter 5: Hidden Treasures and Implications ........................................................................................ 287

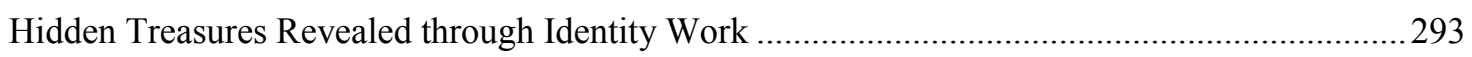

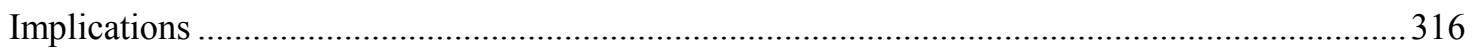

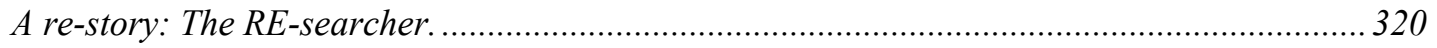

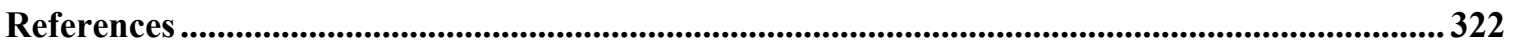

APPENDIX A RECRUITMENT MATERIALS.............................................................................. 342

APPENDIX B INITIAL PHONE SCREENING .................................................................................... 343

APPENDIX C RESEARCH LOG ...................................................................................................... 347

APPENDIX D DATA COLLECTION AND TIMELINE PROCEDURES.................................... 348

APPENDIX E INTERVIEW PROTOCOL ................................................................................................. 349

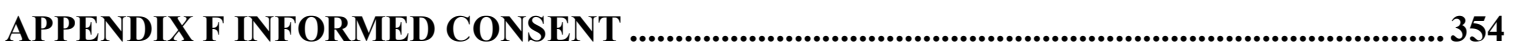

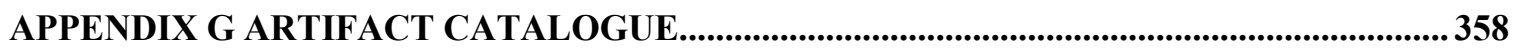




\section{List of Tables}

Table

Page

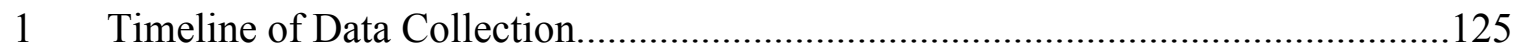

2 Archetypes and Katz Stages and Hidden Treasures........................... 315 


\section{List of Figures}

Figure

Page

1 Ann, Maggie, Miranda, Clara, Joni and Julienne's reuse self-representations.. 153

2 Maggie reuse self-representation (left) and Ann (right)....

3 Joni reuse self-representation (left) and Julienne's reuse self-representation

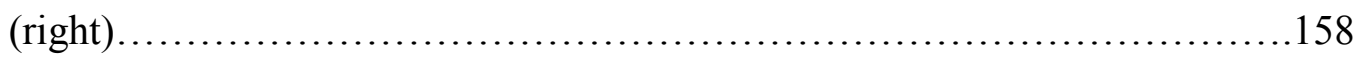

4 Clara's (left) and Miranda's (right) reuse self-representations ...............163

5 Session two dyadic interview..................................... 233

6 Ann's representation of Maggie (left) and Maggie's representation of Ann

(right)

234

7 Clara's reuse portrait of Miranda (left) and Miranda's reuse portrait of Clara (right) .236

8 Maggie's "Looking Through" documentation Part a.......................239

8b Maggie's "Discovering Possibilities in Our Studio" documentation Part b...240

8c Maggie's “Open-ended Materials” documentation Part c...................241

8d Maggie's "Light and Loose Parts" documentation Part d....................242

9 Maggie's "Nora Constructs her Image of Grassy" documentation Part

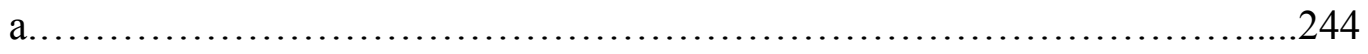

9b Maggie's "Grassy" documentation Part b.................................245

10 Anne's "Fantasy Lands" documentation Part a.........................247

10b Anne's "Reuse Car" documentation Part b.............................248 
10c Anne's "Making Toys from Salvage Materials” documentation Part c........249

10d Ann's "Discard House in Outer Space" documentation Part d................250

11 Ann's "Little Wooden Dolls" documentation Part a.......................251

11b Anne's "Reuse Identity Characters" documentation Part b ................251

12 Clara's "Cardboard People" documentation Part a..........................253

12b Clara's "Body Tracings" documentation Part b...........................253

12c Clara's "Languages of Fiber" documentation Part c.......................253

12d Clara's "Reuse Atelier" documentation Part d...........................254

12e Clara's "Multiple Representations of Selves" documentation Part e...........255

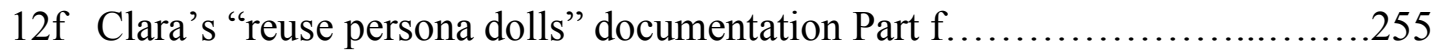

12g Clara's "Girl with the Purple Hair" documentation Part g................... 257

13 Miranda's "Hidden Treasures" documentation Part a.......................259

13b Miranda's “Social Dimensions of Identity" documentation Part b...........261

13c Miranda's "Identity Study" documentation Part c. ........................262

14 Remida inspired persona dolls Logan (left) and Harmony in Dissarray

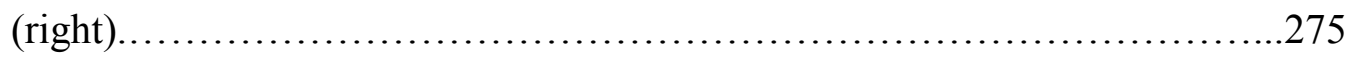




\section{Glossary of Abbreviations}

\begin{tabular}{|l|l|}
\hline UNESCO & United Nations Educational, Scientific and Cultural Organization. \\
\hline DESD & Decade of Education for Sustainable Development \\
\hline EPA & Environmental Protection Agency \\
\hline CDC & Child Development Center \\
\hline ECEFS & Early Childhood Education for Sustainability \\
\hline UNCED & United Nations Conference on Environment and Development \\
\hline IRPP & Inventing Remida Portland Project \\
\hline LatCRT & Latinx Critical Race Theory \\
\hline CRCC & Creative Reuse Cultural Center \\
\hline
\end{tabular}




\section{Chapter I: Introduction}

There is a lack of literature connecting children's social identity formation and education for ecological sustainability in early childhood education. This gap in literature can be attributed to a common misconception that young children are not able to understand and express more complex social identity nor identify with ecological and sustainable education principles (Bennett \& Sani, 2004; Duhn, 2012). However, this study illuminates that from the perspective of Reggio-inspired educators' social constructivist experiences, pre-primary age children are in fact competent and capable of socially constructing complex knowledge (Duhn, 2012; Lesnik-Oberstein, 1994) and of representing their theories on identity and ecology through reuse materials (Reggio Children, 2005).

This qualitative research study involved an examination of six Reggio-inspired educators' perspectives on how they view the construction of social identities with children through repurposed materials such as: 1) a way to see themselves in a material world that is salvageable and reusable, and 2) a way to see their work as an important contribution to society and waste reduction (Helm \& Parnell, 2010; Reggio Children, 2005). The research question was: What are early childhood educators' experiences with reuse materials in Reggio Emilia inspired identity studies?

Developmental psychology is the scientific study of the changes that occur in human beings over the course of their lives. Previous findings in the field show that until the age of 8 , young children's conceptions of social identities focus largely on behavioral and dispositional properties as opposed to belief-based aspects of identity (Bennet, 2004, 
2011; Bennet \& Sanni, 2004). There is a paucity of literature focused specifically on how children construct social identities. In fact, existing research has either focused primarily on the formation of children's individual identities (Bennet, 2004, 2011; Bennet \& Sani, 2004) or has not differentiated between children's personal and social identities (Swanson, Cunningham, Youngblood, \& Spencer, 2009). Traditionally, the construct of a child's ecological self has been largely neglected and this omission may be, in part, because of some researchers believe that social identity constructions are too complex for preoperational cognition (Bennet, 2004, 2011; Bennet \& Sanni, 2004).

However, emerging discourses in early childhood identity theory trend toward the multiplicity of social identities (Brooker \& Woodhead, 2008; Konstantoni, 2011, 2012). A crisscrossing of identities has been used to define the intersectionality of race, gender, sexuality and class identifications. Multiplicity of identities references the entirety of this definition of intersectionality (Hames-Garcia, 2011; Pritchard, 2017) A consequential aim of this research is to narrow the gap in research on ecological identity construction (Davis, 2009; Duhn, 2012; Duhn, Bachmann, \& Harris, 2010; Duhn \& Ritchie, 2014; Elliot \& Davis, 2009; Mackey, 2012).

This study is significant because the emergence of the self is one of the most elemental tasks faced by a young child (Durkin, 1995; Kohlberg, 1966; Lewis, 1990). The infant-toddler and preschool years are a crucial time for identity and language (Copple \& Bredekamp, 2009) as well as foundational years for education of sustainable consumption, reuse, and recycling (Chawla, 2009). In this research study, I introduce alternative thinking into the discourse that dominates early childhood scholarship and 
practice while attempting to avoid the pitfalls of passing judgment on competing theories. I argue that much can be learned by distinguishing the formation of children's social identity from individual identity formation. So much richness can be derived from socially constructivist perspectives related to identity studies which juxtapose mainstream early childhood education approaches. That said, Grieshaber and Cannella (2001) assert that using language to describe developmentalists and re-conceptualists creates a false dichotomy; that both groups share similar backgrounds in education as well as a mutual concern for early childhood education. My research attempts to hold this paradigmatic tension; I do this learning with early childhood educators' experiences of socially constructing identity studies with young children as a Remida-inspired educator.

\section{Statement of the Problem}

When it comes to the emergence of self-awareness, self-concept, and self-esteem in early childhood education, Reggio and Reggio-inspired scholars promote the idea that there exists an inherent value of studying identity with young children (Caldwell, 2003; Dahlberg, Moss, \& Pence, 1999; Reggio Children, 2011). Further, theoretical perspectives associated with Allport (1937), Cooley (1902), Mead (1934), and Lewin (1935) have been used as the foundation for much of the research focused on children's self-development (Bennet. 2004). One common Reggio-inspired principle is that a child's personality and self-image is largely shaped by the time that child is 3 years of age (Gandini \& Edwards, 2001; Gerber, 1998; Pruett, 1999).

Understanding self-development has historically represented the basis for understanding how individuals evolve a sense of personal uniqueness as distinct from 
other individuals (Erikson, 1950). According to Bennett and Sanni (2004), developmental psychologists have almost exclusively treated groups as external to the self. they have viewed group dynamics as merely an influence upon children's selfconcept. In contrast, a social-cognitive perspective advances the idea that "at its heart is an acknowledgment of the interdependence of self and social context" (Bennet, 2004, p. 1). One of the central insights of the social identity approach is that groups can become part of a child's self-concept.

Among developmentalists, the idea of self has been conceived primarily in cognitive terms and a great deal of attention has been given to self-representations (Harter, 1999). Bennet \& Sanni (2004) noted that the developmental study of the self has followed the model of cognitive-developmental theory — specifically Piagetian and neoPiagetian theory. Bennet \& Sanni (2004) reminded us that, "The guiding assumption has been that general conceptual development plays a central role in determining the emergence, form and increasing differentiation of self-conceptions. That is, researchers have sought to account for age-related structural growth in self-conceptions in terms of cognitive ontogenesis" (p.3). Persuasive mainstream developmental discourse conceptualizes self-awareness as when a child cognitively realizes that she is a "distinct individual whose body, mind, and actions are separate from those of other people" (Berger, 2008, p. 190).

Moreover, approaches to identity research with children in group care are often based on developmental literature that suggests, "At about age one an emerging sense of 'me' and 'mine' leads to a new consciousness of others"' (Berger, 2008, p. 190) and that 
the "egocentric" period from 15-18 months "is noteworthy for the emergence of the meself, the sense of self as the object of one's knowledge" (Harter, 1998, p. 562). Overall, developmental psychology has been primarily concerned with the initial emergence of the individual's self-concept rather than the social construction of identities in early childhood education (Brooks-Gunn \& Lewis, 1979; Rochat, 2001).

Early childhood developmental research directed at the aspect of the self-concept referred to as the personal self (the self-defined by idiosyncratic features, such as personality traits, abilities, and preferences) are problematic (Foucault, 1980) from a social identity approach perspective (Tajfel, 1974; Tajfel \& Turner, 1986). Rather, this social identity focus is on the formation of the social self - the self as constructed by one's membership in social groups. Discourses in identity theory warn of the risk of "endorsing the myth of the autonomous and independent person" (Prout, 2011, p. 66) and ignore that people — adults and children alike — live in a complex web of interdependencies (Gallacher \& Gallagher, 2008).

Likewise, social constructivist scholars (Devries \& Kohlberg, 1987; Gardner, 1983; Grieshaber \& Cannella, 2001; Vygotsky, 1978), early childhood education for sustainability researchers (Davis, 2009; Duhn, 2010; Elliot \& Davis, 2009; Parnell, Downs, \& Cullen, 2017), Reggio Emilia pedagogues (Malaguzzi, 1993; Rinaldi, 2006), and Reggio-inspired identity studies academics (Fu, Stremmel, \& Hill, 2002; Gandini \& Edwards, 2001) share the belief that the aim of education, including early childhood education, is to intentionally develop character in both the self and others. This way of educating contributes to the mutual benefit and welfare of the individual and the whole. 
Additionally, emerging pedagogies of place and place-making as a democratic practice are facilitating critical engagement with global issues in early childhood education including how early childhood educators work with the notion of ecological sustainability in their pedagogy and curriculum (Duhn, et al., 2010; Iorio, Hamm, Parnell, \& Quintero, 2017). Increasingly early childhood education for sustainability is looking to global citizenship as a social identity framework for early childhood curriculum and instruction.

Jans (2004) highlighted how contemporary notions of children as citizens, and the social construction of childhood raise interesting questions about children's identities when she stated, "Childhood presents itself more and more as an ambivalent social phenomenon. On the one hand, children are seen as autonomous individuals, on the other hand, as objects of protection" (p. 27). In fact, the social identity approach of acknowledging the interdependence of self and social context is present in global citizenship narratives. Global citizenship is about commitment to justice and wellbeing for all, in the neighborhood as well as on the global scale; it is about the interrelation between self and other in local and global contexts (Dahlberg \& Moss, 2005; Duhn et al., 2010).

Regarding the origins and development of self and identity, relatively little work in developmental psychology has addressed children's identities (Bennet, 2004, 2011). According to Bennet and Sani (2004), the lack of historical research on childrens' social identities can be attributed to infant and developmental psychologists' contentions that complex social identity constructions - the self as defined by group memberships, such as 
ethnicity, religion, gender, and subcultural groups - are too abstract for preprimary school cognition (Quintana, 1998). These same developmental perspectives hold that young children's conceptions of social identities are based on external characteristics and social relationships (Bennet, 2004, 2011; Quintana, 1998) and only by late childhood are social identities conceived with reference to stable trait-characteristics and beliefs (Livesley \& Bromley, 1973; Newman \& Rouble, 1988; Bennet, 2004, 2011).

Quintana's (1998) stage model suggests that after the age of 10, a more sophisticated understanding of social categories develops in children. According to this model, the primary focus is on individual identity in early childhood and related curriculum often encourages individualism.

Critiques of such narrow interpretations of identity in early childhood challenge mainstream regimes of truth (Foucault, 1980) that perpetuate a Saussurean binary opposition between individual and social identity. In fact, Brewer and Gardener (1996) and Thoits and Vissup (1997) have posited that through this Saussurean opposition perspective, individual identity assumes dominance over social identity and this ordering reproduces the notion that individualism is superior to collectivism. That said, more recent discourses in early childhood identity theory trend toward the multiplicity of social and collective identities (e.g. Brooker \& Woodhead (Eds.), 2008; Konstantoni, 2011, 2012; Raburu, 2015). These sociocultural discourses are relevant to a scattered, diverse and mobile society, to the sociology of early childhood, and to a growing awareness of the importance of the early years in childhood. 
Further, Kohn's (2005) criticism of individual competition and rewards in education, advocated for acknowledging a necessary balance and interdependency between individual and social identities. Kohn said, "Community is not enough; we need autonomy too. In fact, when both of these features are present there is another way to describe the arrangement that results. It is called democracy" (p. 119). Like the Reggio Emilia approach, Kohn believes an educator's responsibility toward children is also political.

According to Brooker and Woodhead (2008), identity simultaneously covers two core human motives: the need to belong and the need to be unique. Many social constructivist early childhood educators believe that from the time of birth, every child begins the process of socially constructing a uniquely personal and ecological identity that is marked by a growing awareness of the cultural importance of markers such as gender, ethnicity, age, and status within the child's immediate community (Brooker \& Woodhead, 2008). For example, Bennet and Sanni (2004) suggested that it is precisely in the process of socially constructing identities how social constructivist educators, in socializing children, may give belief-based identities priority, thus scaffolding to a greater degree ecological and value-based social identity.

Studies theorizing that younger children think of social identities primarily in behavioral terms (e.g. "Girls play with dolls") and that the very young are incapable of recognizing the importance of shared beliefs and values (e.g. gender stereotypes) may be reflective of how the sorts of identities that children understand well (e.g. gender, age, and so on) are perceived by adults (Grace, David, \& Ryan, 2008; Raag \& Rackliff, 1998). 
These are ascribed identities that parents and educators commonly give priority to when scaffolding the guidance provided to young children to a greater degree than those reflecting personal choice (Aina \& Cameron, 2011; Barron, 2007; Giraldo \& Coylar, 2012; Skattebol, 2006). For example, a Russian parent who is a professor of Russian studies might scaffold the importance of national identity (e.g. Russian people believe in neighborliness) for a 4 year-old even though developmental research states such young children can't comprehend shared beliefs and values.

One of the most important contexts in which social identities are produced, reproduced, and disrupted are in preprimary schools where learning communities develop self-awareness through formal and informal curriculum (Giraldo \& Colyar, 2012). However, identity studies in early childhood education are often reproduced and/or disrupted by the constraints of developmental psychologists' (Brooks-Gunn \& Lewis, 1979; Rochat, 2001; Quintana, 1998) assertions that value-laden social identity constructions are too complex for infant, toddler, and preschool cognition and curriculum.

Brooks-Gunn and Lewis (1979), Rochat (2001) and Quintana's (1998) research findings have shown that young children's conceptions focus largely on behavioral and dispositional properties as opposed to belief-based aspects of social identity until the age of 8. By extension, researchers such as Davis (2009) and Duhn (2010) have noted that the belief-based aspects of ecological identity formation are generally considered too complex and inappropriate for early childhood education practice. 
In contrast, an alternative educational viewpoint offered through the research in the Municipal Infant-Toddler Centers and Preprimary Schools of Reggio Emilia, Italy came through an ongoing cultural project in their Remida creative recycling center that had been explicitly ascribing high value to the use of waste materials as a part of young children's educational experiences since 1996 (Reggio Children, 2005). The optimistic underlying point of view on reversing environmental degradation through Remida pedagogy provides children with opportunities to co-construct complex social identities in Reggio’s Municipal Infant/Toddler Centers and Preprimary Schools. The approach involves actively engaging children and educators in hands-on experiences that approach ideas related to environmental sustainability work with reused materials.

During a recent Reggio study tour, Wexler (2004) noted,

Our collective wisdom was challenged by what we saw. We had believed that the young child is egocentric; that she cannot share, cannot understand the point of view of others, and that she has a short attention span. And finally, we realized that our curriculum is shaped by these assumptions" (p. 14).

The newly emerging wisdom influences fresh ways of conceiving curricula and re-conceptualizing pedagogy, the relationship between children and the planet, and relationality in terms of children's capacity for social identity formation and identification as global citizens.

The stories of Remida and Reggio-inspired early childhood educators socially constructing identities with children through the use of reuse materials are being made increasingly visible in early childhood education literature (Cadwell, 2007; Eckhoff, 
Hallenbeck, \& Spearman, 2011; Eckhoff \& Spearman, 2009; Guerra, 2013; Guerra \& Zuccoli, 2014; Helm \& Parnell, 2010; Hobba, 2006; Kelly \& Lukaart, 2005; Montoya, 2009; Parnell, Downs, \& Cullen, 2017; Odegard, 2012; Project Zero and Reggio Children, 2001; Reggio Children, 2005; Sharova, 2012; Wexler, 2004; Tarr, 2001, 2003, 2008). One example of how children's perspectives are influencing emerging pedagogy is recent research that positions the child as bricoleur — a French term describing a person who uses the tools that are available to complete a task. This image of the child as bricoleur conceives of the child as a co-researcher who is capable of inventing methodologies for collecting data on early childhood education for sustainability (Iorio, Hamm, Parnell, \& Quintero, 2017).

Problems arise from individualistic ways of thinking that influence early childhood pedagogy. Emerging pedagogy addresses the problems that arise from perceiving oneself as disconnected from the community of nature including human and more-than-human members (Latourre, 2004; Taylor \& Pacini-Ketchabaw, 2015). A foundation of knowing that we are part of the web of the community is important for both the period of early childhood and for future learning. In this way, pedagogy mobilizes complexity and relationality which will empower and prepare children to be resilient when the news about the condition of the planet is introduced. Early childhood education for sustainability scaffolds for the formation of creative thinking and problem solving, communication skills, and art as a medium for deep self-reflection as a member of something bigger than oneself. 
To build shared meaning early in this dialogue, I have developed a series of definitions around key terms and concepts used in this study. These terms are presented in alphabetical order in the following subsection followed by an extended conceptualization of multi-metaphorical meta-frameworks. I engage multiple frameworks such as architecture as a metaphor for deconstructionism and borderlands as a metaphor for identity studies as a means for thinking about using creativity to foster connections between individual and social identities with reuse materials.

\section{Key Terms and Concepts}

I begin this subsection with a definition of the term archetype which I use to interpret my narrative data. According to Clandinin (2007), narrative interviews about life stories are a methodology that yields data that are both unique-to-the-individual and universal-for-the-individual (as in the common motifs and archetypes experienced). In Jungian psychology, an archetype is an inherited pattern of thought or symbolic image that is gleaned from the past collective experience of human cultures and is present in the unconscious of the individual.

The second definition is for the conceptual term Borderlands theory. For the purpose of this study, I seek to contribute to a better understanding of Gloria Anzaldua's (1993) notion of the Borderlands in both its geographical and ideological dimensions situated within the context of identity studies. Anzaldua's Borderland theory is a project of resistance formulated as a set of processes aimed to guide the inner life of a colonized person in the struggle to achieve decolonization and liberation (Orozco-Mendoza, 2008). 
The third definition is for the term collectivist identity: One's identity is, in large part, a function of one's membership and role in a group, e.g., the family or work team. The survival and success of the group ensures the wellbeing of the individual so that by considering the needs and feelings of others, one protects oneself. Harmony and the interdependence of group members are stressed and valued. Group members are relatively close psychologically and emotionally, but distant toward non-group members.

The fourth definition is for the term early childhood education for sustainability. According to the United Nations Educational, Scientific and Cultural Organization (UNESCO) (2008), early childhood education for sustainability encompasses more than traditional environmental education. The global organization suggests sustainability education must be broader than simply taking children outdoors to discover the beauty of nature and speaking to them about the natural environment. It must involve opportunities for children to actively engage in intellectual dialogue regarding environmental and social sustainability and physically participate in concrete actions in favor of the environment. In addition, sustainability education should incorporate learning that is compassionate and respectful of differences, equality, and fairness as the world is increasingly interdependent and inter-connected. UNESCO (2008) calls for education to emphasize "7Rs" for sustainable development: reduce, reuse, recycle, respect, repair, reflect, and refuse.

Next is the working definition of the term early childhood development as used in this study followed by the definition of the term early childhood educator. The term early childhood development as defined by Katz (1996) refers to "a set of concepts, 
principles, and facts that explain, describe and account for the processes involved in change from immature to mature status and functioning” (p. 137). The term early childhood educator refers to a professional who works directly with young children in childhood care and education settings who generally self-identify their work as preschool teacher, childcare provider, early childhood teacher or educator, or after school provider. Early childhood research primarily uses the terms infant teacher, preschool teacher, practitioner, early childhood teacher, or educator (Katz, 1995; Parnell, 2010). For the purposes of this study, I define early childhood educator as individuals who provide direct services to children from birth through 5 years of age in the early childhood community.

For the purposes of this dissertation, I am replacing the institutional discourse terms workforce and field with the term learning community. The term learning community conveys a level of connection and interdependence between members of a group that can construct meaning together. This construction of meaning together is at the heart of open dialogue between participants of a community (Cadwell, 2003). All of the study participants involved in this research are therefore deemed early childhood educators. I will refer to them as participants hereafter to distinguish their specific roles in this dissertation from the generalized use of the word early childhood educator found in the literature typically used to represent the larger early childhood education community (Katz, 1995).

What follows are the definitions for three terms central to this study: ecology, ecological identity, and education for sustainability. Ecology refers to the scientific 
study of the relationship between organisms and their surrounding environment (Pena, 2005). Devon G. Pena, whose intellectual mentorship during my graduate education encouraged me to see that ecology and Chicanx studies are not only deeply connected but are in fact "subversive kin". Pena $(1998,2005)$, whose research interests include ethnoecology, agroecology, and the anthropology of place and place-making, writes extensively about how a lack of connection between culture, ecology, and learning exists in Chicanx studies as a field. Pena (1998) warns that "lacking an epistemology of local knowledge, students of Chicana/o studies will be left few options for critically approaching and perhaps reversing the politic-economic processes that destroy places" (p.11). Ecological identity refers to the social construction of a profound sense of oneself in relationship with natural and social ecosystems (Thomashow, 1995). Education for sustainability refers to the wider perspective of environmental education that combines ecological principles with social justice principles (Mackey, 2010). Increasing literacy in ecological, economic, and social sustainability is emerging in society and academia. Discourse around the concept of "Just Sustainability," is emerging in transnational community groups. This suggests the need for a parallel dialogue within the community of early childhood education.

Central to this study is the metaphor of a hidden treasure. Over time, as I reflected on the interview data I collected for this study, I began to realize that each participant brought a unique perspective to the project, whether individually or through sharing stories and I began to call these unique points of view, hidden treasures. The term was introduced through participant Miranda's documentation of Remida-inspired 
identity studies. The documentation was inspired by Elizabeth Gilbert's (2015) book "Big Magic" and the idea that we all have Hidden Treasures inside of us that we can reveal through identity work.

Next is the definition of another term central to this study: identity. Identity is an agentic core of personality, as opposed to cognition, by which humans learn to increasingly differentiate and master themselves and orient themselves in relation to the world. This concept gives meaning and purpose to life and perspective to human efforts. Through identity, individuals come to situate themselves, for instance, as belonging to a distinct race, place, ethnicity, nationality, gender, or culture. Identity simultaneously covers two core human motives: the need to belong and the need to be unique (Brooker \&Woodhead, 2008).

A related, more specific term is that of individual identity. Here, this term is defined as children's subjective feelings as opposed to constructed beliefs, about their distinctiveness from others and their sense of uniqueness, of individuality. Individual identity is achieved by differentiating from others and focuses on those aspects of the self-concept that differentiate the person from other persons. Each person is a unique constellation of traits and characteristics.

The next term is that of individualism. A person identifies primarily with self; one's primary interest is with their own needs being satisfied before those of the group based on the assumption that looking after and taking care of oneself, being selfsufficient, guarantees the well-being of the group. Independence and self-reliance are greatly stressed and valued. In general, those who prioritize an individuated perspective 
tend to distance themselves psychologically and emotionally from each other. One may choose to join groups, but group membership is not essential to one's identity or self-concept of success (Triandis, 1995).

Here I provide a definition for the term Metaphor. Sfard (1998) considered figurative language or metaphors for learning to be "the most primitive, most elusive, and yet amazingly informative subjects of analysis. Their special power stems from the fact that they often cross the borders between the spontaneous and the scientific, between the intuitive and the formal" (p. 4). Integral to Reggio's social constructivist practices are the concepts of semiotics and the use of metaphor (Vecchi, 2010) as they aid in the construction of symbolic thinking, social interaction, and cultural awareness.

Here I provide a definition of the term Reggio-inspired educators. To say Reggio- inspired regarding early childhood programs is an acknowledgment that one does not copy the Reggio approach per se. Instead, educators outside of Reggio Emilia, Italy experiment and re-interpret the principles that offer useful reference points in a way that is locally relevant and culturally appropriate (Wien, Gyuvesky, \& Berdoussis, 2011). For instance, in the system of Municipal Infant-Toddler Centers and Pre-Primary Schools in Reggio Emilia, Italy, the role of director does not exist in the centers or schools. The administrative equivalent is in government buildings and operates from those locations, whereas in North America most Reggio-inspired child development centers have a director and administrators on site.

Next is the definition of the Reggio Emilia experience used in this study. Since the late 1940s, the Municipal Preprimary Schools and Infant/Toddler Centers of Reggio 
Emilia's system has evolved their own unique set of philosophical and pedagogical assumptions, methods of school organization, and principles of environmental design that, taken as a unified whole, have been termed the Reggio Emilia experience (Edwards, Gandini, \& Forman, 2012). The Reggio Emilia experience intentionally works to foster children's intellectual growth through a systematic focus on symbolic representation. Young children are encouraged to explore their environment and express themselves through multiple paths and languages, including the expressive, communicative, symbolic, cognitive, ethical, metaphorical, logical, imaginative, relational, and digital languages (Remida Series, 2016). A fuller description to be found in Chapter 2.

Reggio Emilia philosophy refers to the relationship and community-based education was developed by a set of schools in Reggio Emilia, Italy. The philosophy has evolved since its inception in the late 1940s. Educators in Reggio Emilia, including cofounder Loris Malaguzzi, have been inspired by early childhood psychologists and philosophers such as Dewey, Piaget, Vygotsky, Gardner, and Bruner (Edwards, Gandini, \& Forman, 1998). For more on the making of the philosophy and pedagogy of these schools see Barrazzoni’s (2005) works. Also, Chapter 2 provides a more detailed description of Reggio Emilia and Remida approaches.

Now I will provide a definition of a Remida Centre, the context of this study. The name Remida (harkening the name King Midas) refers to a creative recycling center for reclaiming goods. The original Remida location is geographically located in the city of Reggio Emilia, located in northern Italy in the province of Emilia-Romagna. However, there are now 17 Remida centers worldwide, located in places such as Denmark, 
Germany, and Australia. The idea of Remida can be considered an alternative educational viewpoint and is a conceptual, educational, and environmental space to learn about repurposing material goods for educational purposes (Parnell, Downs, \& Cullen, 2015, 2017; Reggio Children, 2016).

Found with the Remida centers are Remida Reuse/Reclaimed/Repurposed Materials, which, refer to alternative and reclaimed materials obtained from the unsold stock and scrap materials of industrial and manufacturer production with the aim to reinvent their use and meaning. "There are now 170 companies that contribute their discard materials; paper, metal, cord, leather, fabrics, upholstery, food containers, bottles, and so on. Materials that are carefully inspected and selected by Friends of Reggio Children Association, then transported to the Remida headquarters by Iren/INIA — a multi-utility provider. In Remida the objects are received, displayed, and then offered in a sort of emporium or warehouse of wonders" (Reggio Children, 2005). The data for this dissertation was collected at a Remida-inspired cultural education center in Oregon.

What follows is the working definition of Social Constructivism. There is currently no generally accepted definition of the concept and process of social construction found in psychological or educational literature. Instead, definitions are diverse and depend on the theoretical context in which they are embedded. The concept of co-constructivism challenges traditional (i.e. Western) epistemological constructivism and is grounded in symbolic interactionist perspectives (Mead, 1934) and sociocultural theory (Vygotsky, 1962). Many social constructivists share the value that learning is inherently a social activity; learning and enculturation are not bounded by cognition but 
are intrinsically social endeavors ingrained in a society and reflecting its knowledge, perspectives, and beliefs. Children socially construct their knowledge, not only from direct personal experience, but also from being informed by others and by being shaped through social experience and interaction.

Bruner (1986) informed understanding of the term in the following way, Most learning in most settings is a communal activity, a sharing of the culture. It is not just that the child must make his knowledge his own, but that he must make it his own in a community of those who share his sense of belonging to a culture (p. 86).

Here, knowledge is no longer seen as solely residing in the mind of everyone, but as being distributed across individuals whose joint interactions and negotiations determine decisions and solutions to problems (Hutchins, 1995).

Critical to this study is the concept of social identity. This term refers to ways in which children construct who they are (or would like to be) as being the same as others, typically through identification with family and/or peer culture. Erikson (1902-1994) became one of the earliest psychologists to take an explicit research interest in identity. The framework he developed is based on distinguishing between the psychological sense of continuity, known as the ego; the personal quirks that separate one person from the next, known as the personal identity; and the collection of social roles that a person might play, known as either the social or cultural identity. 
The term sustainability refers to a complex and highly contested concept, that human activities are organized in a manner that does not damage the environment, reduce biodiversity, or undermine ecosystem integrity (Pena, 2005).

Finally, here I define the term theoretical frameworks. The theoretical frameworks I have chosen consist of concepts, their definitions, and references to relevant scholarly literature and existing theory that has been relevant for this study. I have applied multiple theoretical frameworks to interpret the meaning, nature, and challenges associated with the phenomenon of social constructivist experiences with identity studies, so that we may use that knowledge and understanding to enhance and extend identity studies. This concludes the set of key terms related to this study.

Now that I have explored the evidence and significance of the problem and key definitions, there are a few other theoretical contexts I need to examine to better understand my literature. These areas will include multi-metaphorical meta-frameworks including architecture as a metaphor for social constructivism and Borderlands as a metaphor for plural identities. The following subsection presents how multiple metaphors illuminate my interpretation of the data I collected so I can draw on my participants' epistemological curiosity as well as my own to pursue this research.

\section{Metaphors and Meaning Making}

Socially constructing knowledge in early childhood education lends itself to the methodological flexibility of multi-metaphorical meta-frameworks (Sfard, 1998) in communicating unconventional data and theories. Sfard (1998) engaged metaphor to reach the core levels of our thinking and to make transparent the tacit assumptions and 
beliefs that guide us. Her use of multiple metaphors for learning was inspired by the concept that "new knowledge germinates in old knowledge" (p. 4) an idea she noted, that has been embraced by theoreticians of knowledge construction, from Piaget to Vygotsky to present-day cognitive scientists. I introduce an architectural metaphor alongside the Borderlands metaphor as a tool to situate the problem of the social constructivist approach to identity formation through an interpretation of its historical, social, and cultural contexts.

First, I apply an architecture metaphor to illustrate how using creativity to facilitate the social construction of self and social identities using reuse materials relates to multiple metaphors employed in this study. Here, architecture as metaphor illuminates the significance of Remida-inspired identity studies in early childhood education for sustainability.

\section{Architecture as a metaphor for social constructivism.}

The architectural origins of the Municipal Preprimary Schools of Reggio Emilia are reconstructed in a book on the history of the first school, "XXV Aprile" People's Nursery School of Villa Cella. As the founder of Reggio Emilia's educational philosophy, Malaguzzi described his first impression of the post-World Was II school being built by partisans: "There were piles of sand and of bricks, a wheelbarrow full of hammers, shovels and hoes. Behind a curtain made of rags to shield them from the sun two women were hammering the mortar off the bricks" (Barrazonni, 2005, p.13). Barrazonni (2005) pointed out that the municipal preprimary schools of Reggio Emilia were built directly from the rubble of World War II materials, which, she argued 
contributed to the development of a cultural value of building in a sustainable way, an idea that is both new and old:

Sadly enough, building material was everywhere, thanks to the Allied bombings which had decimated entire neighborhoods, like the one around the Reggiane, a local factory, and the one near the airfield. Scavenging for bricks, beams, door and window frames, and even a complete stone staircase sustained the project until its completion towards the end of 1946. (Barrazonni, 2005, p.21)

Architecture as a metaphor for claiming space and making a new culture as expressed by Borderlands theorist Anzaldua (1987) is introduced here through Chicanx studies and the historical context of the founding of the Municipal Schools of Reggio Emilia. I extend architecture as a metaphor to what I consider to be the cornice stone of Reggio Emilia constructions of philosophical thought—Remida centers, which, 70 years later are socially constructing ecological consciousness in young children and educators, out of the contemporary physical rubble of hyper-consumerism—reuse and salvage materials. Whereas the founders of Reggio Emilia education were building physical space to transmit democratic principles during the decline of fascism of Italy, contemporary Reggio-inspired educators are designing physical and conceptual spaces to foster democratic and ecological principles. Parnell, Downs, and Cullen (2017) demonstrate how both principles come into play through his Remida-inspired research:

This project on shiny fish pushes the founders out of our comfort zone and into a space 
where the values of our places, spaces, time, and commitment to postanthropocene are critical to the nature of proceeding toward a new ecology of relations between place, multispecies, materials, children, teachers, and community. (Parnell, Downs, \& Cullen, 2017, p. 10)

As related, the field of Chicanx studies heavily engage deconstructionist semiotic interpretations. The philosophy of deconstructionism emphasizes the relational quality of meaning and inspired the movement of deconstructivist architecture and art. Building on what was said above, deconstructivism is a development of postmodern architecture that began in the late 1980 s and is influenced by the theory of Deconstruction. It is characterized by fragmentation and an interest in changing a structure's surface and design in a way that appears to distort and dislocate elements of conventional architecture. The finished visual appearance of buildings that exhibit deconstructivist styles is characterized by unpredictability and controlled chaos. A presupposition of this study is that architecture is a language capable of communicating meaning and of adapting to methods of linguistic philosophy (Curl, 2006). In this study, I draw on both architecture in the literal sense of involving educators in designing self-portraits as a means of self-exploration and social reflection, and as a metaphor for how Remida inspires a different way of thinking about how to facilitate learning by using repurposed materials. 


\section{Borderlands as a metaphor for identity studies.}

Chicana cultural theorist Anzaldua (1987) suggested that in the process of straddling two cultures, a third culture is created. She draws a powerful constructivist metaphor:

So, don't give me your tenets and your laws. Don't give me your lukewarm gods. What I want is an accounting with all three cultures - white, Mexican, Indian. I want the freedom to carve and chisel my own face, to staunch the bleeding with ashes, to fashion my own gods out of my entrails. And if going home is denied me then I will have to stand and claim my own space, making a new culture-una cultura mestiza - with my own lumber, my own bricks and mortar and my own feminist architecture. (p. 44)

Similarly, architectural metaphors for learning lend themselves equally well to both deconstructionist and constructivist pedagogies that value drawing on both the new and old to create a brand new, third space that makes possible reconciliation and integration by moving "toward a more whole perspective, one that includes rather than excludes" (Anzaldúa, 1987, p. 79). The use of metaphor as a conceptual tool for explaining the cognitive processes that turn old into new is particularly apropos for embracing the complex and shifting landscape of reuse, social identities, and learning at a glance (Reggio Children, 2005). Similarly, learning communities involved in Chicanx studies tend to heavily engage deconstructionist semiotic interpretations. Deconstructionism, as aforementioned, can be described as a theory of reading in adult education that which aims to undermine the opposition within texts. Anzaldua (1987) stated, 
A border is a dividing line, a narrow strip along a steep edge. A borderland is a vague and undetermined place created by the emotional residue of an unnatural boundary. It is in a constant state of transition. The prohibited and the forbidden are its inhabitants (p. 25).

Borderlands theory is a feminist and decolonial framework that deconstructs the formation of a colonial identity through re-signifying and recovering self-affirming complex images of female identity as a decolonial practice.

As part of analyzing the educational significance of addressing the lack of research on children's social identity and early childhood education for sustainability, I want to clearly delineate the educational significance of the problem, including issues of equity. Chicanx studyists such as Vila (2003) critique Borderlands epistemology and have suggested that it silences, displaces, and essentializes Mexican-origin migrants by using "border crossing” as a metaphor for a new paradigm of intercultural experience. As a self-conscious researcher, I acknowledge that the metaphorical use of Borderlands as a conceptual migration is problematized here. As Anzaldua (1990) noted:

We preserve ourselves through metaphor; through metaphor we protect ourselves. The resistance to change in a person is in direct proportion to the number of dead metaphors that person carries. But we can also change ourselves through metaphor. And, most importantly, we can share ourselves through metaphorattempt to put, in words, the flow of some of our internal pictures, sounds, sensations, and feelings and hope that as the reader reads the pages these "metaphors" will be "activated" and live in her. (p.121) 
Emerging from my own Borderland experience, this dissertation situates the problematizing of conventional early childhood education identity theory within the context of architecture as a metaphor for constructivism and Borderlands as a metaphor for bridging identities. As Anzaldua (1999) reminds us about this very topic,

An image is a bridge between evoked emotion and conscious knowledge; words are the cables that hold up the bridge. Images are more direct, more immediate than words, and closer to the unconscious. Picture language precedes thinking in words; the metaphorical mind precedes analytical consciousness (Keating (Ed.), p.91).

The use of metaphor is well suited to early childhood education, social constructivism, recycling, and Chicana studies and to this researching line of inquiry.

In sum, I have written to the value of metaphors for learning (Anzaldua, 1987;

Rinaldi, 2006; Sfard, 1998) and how socially constructing knowledge in early childhood education lends itself to the methodological flexibility of "multi-metaphorical metaframeworks" in communicating unconventional data and theories. Now that I have shared key terms, definitions, and concepts that elucidate this research study, it is time to look more deeply into how I positioned myself in this research, work, and thinking.

\section{Positionality Statement}

As a female of Mexican descent, born in the United States, with a politicized identity, I position myself within Chicana Borderlands cultural theory (Alarcon, 1996; Anzaldua, 1987; Donadey, 2007; Elenes, 2011; Herrera-Sobek, 2006; Keating, 2009; Rebolledo, 2006; Solis Ybarra, 2009). Chicanx studies is an interdisciplinary 
community-centered activist scholarship influenced by deconstruction theory, a form of philosophical and literary interpretations that denies the possibility of essential or intrinsic and stable meaning or unmediated access to reality; identity is viewed in nonessentialist terms as a construct (Derrida, 1967). Like social constructivism as a philosophy, the Reggio Emilia approach to early education, and the Remida approach, deconstructionism is not a method. Derrida is careful to avoid the term "method" because it carries connotations of a procedural form of judgment. An educator with a method has already decided how to proceed and is unable to be open minded and epistemologically curious about the matter of thought at hand. For Derrida, this is irresponsibility itself. Thus, to talk of a method in relation to deconstruction, especially regarding its ethical-political implications. would appear to go directly against the spirit of Derrida's philosophical stance (Beardsworth, 1996). Chicanx studies see history as a series of narratives; therefore, the task of the Chicanx studyist is to deconstruct the systems of thought that frame socially constructed stories.

Further, Borderlands theory is a feminist perspective that emerged from Chicana studies. It is a language that explains the social conditions of individuals with hybrid identities and moves beyond binary constructions. A Borderlands paradigm conceptualizes individual and social identities as interdependent and fluid. Keating (2009) describes the theory as such,

For Anzaldua (1987), Borderlands, with a capital B, represents a concept that draws from yet goes beyond the geopolitical Texas/Mexico borderlands to encompass psychic, sexual, and spiritual Borderlands as well. These 
B/borderlands — in both their geographic and metaphoric meanings_-represent intensely painful yet also potentially transformational spaces where opposites converge, conflict, and transform (p.318).

I embrace an integrative approach to identify studies that socially constructs individual selfhood "me" in early childhood within the context of social selfhood "we." Chicana studies learning, and teaching practices demonstrate how cultural productions are sites where epistemological forms of domination can be contested and recreated in ways that allow for the creation of alternative identities and subjectivities. Padilla and Martinez (2005) note when we "challenge the political and ideological aspects of canonical science, especially social science... we end up with a crisis in epistemology" (p.211). They argue this has been true for various minority groups and women and is particularly applicable in terms of developing world perspectives. Because of such an epistemological crisis, one has to take the path that Freire (2001) indicated with the term "epistemological curiosity" (p. 83). In creating this concept, Freire was signaling a way to develop and explore new and evolving epistemological spaces (Padilla \& Montiel, 1998, p. 211). Similarly, I am exploring how Remida Reggio locates its practices in early childhood education on sites where dominant constructions of early childhood identity can be contested in creative ways that invite the social construction of a multiplicity of identities.

\section{Prologue}

To more fully illuminate my alternative point of view of collectivism in early childhood education classrooms, I offer a prologue and reflective component of pre- 
research that brings me to this area of study. The following prologue is derived from a content analysis of my first-year teaching journal at a Reggio-inspired preprimary school in Portland, Oregon. My journal was a source of data I used to identify three characteristics of knowledge that have been generated by self-study research for an independent study. Beck, Freese, and Kosnik (2004) help me to sum up my reasons for offering a prologue,

As educational researchers and reformers, in our ongoing search for ways to improve teaching and learning, it is important to look backward to take stock of our beginnings, where we have been, what we have accomplished, and what we have learned (p. 20).

Their thoughts about looking back to improve our teaching and learning allow me to offer my background in an effort to center my research statement as a co-protagonist in my early childhood community, bringing my community's voice to the fore and helping to define me through this "we" prologue.

June 2012

During my first year "survival stage" of teaching at a Reggio Emilia inspired private preschool, my lead teacher, director and I had a conference with a samesex parent family regarding concerns about their son's identity. The mothers related to us how strongly their son identified with a classmate whom they considered to be a negative influence and their struggles at home with challenging behaviors that they considered uncharacteristic of their son. My lead teacher Joe shared examples of how we respond with positive discipline to challenging 
behaviors at school. I shared how I have frequently noticed friendships develop between children with different temperaments. Children like her son, who are more cautious and slower to warm are often drawn to feisty and highly active friends with whom they want to identify. I then suggested she engage in "identity work" to help him construct a stronger sense of his own identity; perhaps helping him to fill out a traditional All About Me book. We discussed having a classroom circle time to discuss the significance of his name and to use his birthday as an opportunity to celebrate his unique self. His mom said "Even at open play events outside of school, he always is attracted to extroverted, feisty boys. I like the idea of the identity work because the more he develops his own identity the more he will be able to stand on his own if he disagrees with a friend's behavior." My lead teacher (who identifies as a radical constructivist) and I began to collaborate on the novel idea of doing "identity work" in the classroom; we wondered: can preschool age children understand the concept of social and personal identity development? Are any of their interests truly personal and not developed through social relationships? Why do some children identify mostly socially or individually? Are personal interests in mermaids vs. spaceships based on gender membership identity? How do the children subjectively identify with school membership? If we were to be intentional about social and personal identity development would it affect children's behavior or cognition? How can we involve families in exploring social identity? We decided I would start an "All about my character" story workshop in small groups and invite children to 
develop their own characters. In anticipation of these small groups, I began researching" identity work "and "early childhood" which led both to "All About Me" curriculum and a developmental psychology article by Mark Bennet (2004) on the paucity of research on children's identities. Bennet (2004) states,

The structures and processes underlying the self and human identity are as fundamental to psychological inquiry as it is possible to imagine. These topics have occupied social psychology's centre stage for a century, but interestingly, the same cannot with respect to developmental psychology. Despite significant early statements from Baldwin (1895), James (1890), Cooley (1902), and Mead (1934) on the origins and development of self and identity, relatively little work has addressed children's identities. (p.512).

As I pursue this line of inquiry, I realize I have focused on identity formation and learning in graduate-level auto-ethnographic and self-study research. A reference to Tajfel and Turner in Mark Bennet's article on "Children and Social Identity" looks familiar because I cited them in graduate research papers on learning as a culturally shaped activity.

August 9, 2012

Today I did individual interviews during small group time, based on questions from an online "All About Me" curriculum packet:

- What is your first and last name?

- Traits that describe you?

- Things that you are afraid of?

- Things that you love?

- Things you are good at doing?

- People or places that you would like to see?

- Dreams that you have?

The first interview with eight-year-old visiting sibling alum was amazing but my tape recorder volume was off. I asked her if she would do it again, but she didn't 
want to. The four remaining children colored while I "interviewed" each child, which I described to them as "when a person asks another person question about themselves." I asked the normally boisterous group to be quiet so that the tape recorder would pick up our conversation. I noticed they were listening very quietly, knowing that their turn was next. A five-year-old went next and I asked, "What is your name?" I realized this was a difficult question for this child because he was transgender and had recently changed from a female to male name. How could I explore this sensitive topic with him? He was very imaginative and sincere in his responses to the questions that followed. I noticed the cross-pollination of children's responses based on having overheard previous interviews. I thought to myself, maybe this is what they mean by the teacher as researcher? I was aware of how significant my relationships with the children were to how they responded to the interviews. One child did not want to participate, and I wondered if this was because I do not have a closer bond with her? What would interviews have been like had they been conducted in private? October 27, 2012 Joe and I were both impressed with the identity studies made visible through mixed collage self-portraits during professional development at the Reggioinspired Opal School. Our small groups had difficulty answering the "All About Me" questions, so we invited the children to respond to the provocation "The important thing about me is..." which was inspired by our reading of Margaret Wise Brown’s “The Important Book.” The self-portrait collages seemed like a 
natural extension of this inquiry, so we set out a variety of collage materials including miniature Lincoln Logs, primary color wooden geometric shapes, beads, multi-colored cotton balls, pastel sea glass, and assorted plastic figures. The early morning small groups were the first to encounter the materials, and I was very hands-off on the first few self-portraits. The materials were exhibited on one of the longer tables, and individually they were offered simple instructions to engage in the activity. I set out an example of one I made of myself, and gently reminded children to look in the mirrors from time to time in order to construct a representation of their own features. Although the first few three-year-old students chose materials seemingly haphazardly_placing them in random order on the circle corkboards-I decided not to 'correct' them and appreciated with them, the beauty and intricacy of their symbolic self-representations and designs. The next group of children who approached the activity was older and has practiced drawing facial features and drawing images of themselves while looking in mirrors since last year. I noticed that these four-year-old children looked at their reflections for longer periods of time. Then they organized the collage materials on the circular corkboards in ways that demonstrate their developmental stage of representational thought, and ability to create representations from observation. In those moments, I became aware of a spectrum of developmental stages and abilities reflected in the self-portraits, which were revealed through the initial open-ended approach to the activity. 
The last few self-portraits were interesting to facilitate because they took place over the course of a week and some children approached the provocation having already seen their friends' work. At least one "example" of a self-portrait done by a student was available to look at on the table. The activity became increasingly collaborative; we were working side by side, reflecting on each other's portraits. As a co-learner, I began to experiment with comparative language by encouraging younger students to continue to look into the mirror as they worked, to describe what they saw, and then to choose corresponding materials to represent their eyes, nose, mouth, and eyebrows. The following questions encouraged comparative language; "Where does your nose belong on your face? Does it belong above or below your eyes?"

This project has been very meaningful as an extension of our developing exploration of individual and social identity in our classroom community; we intentionally situated individual identities "me" in the context of social identities "we." Reflecting on the experience together with my lead teacher has expanded my understanding as a student teacher of the ways we can assess developmental stages of representational thought and comparative language through identity studies, and the value of scaffolding during small group identity studies. It has been especially meaningful to observe parents and children work side by side and reflect on the self-portraits together. Parent's curiosity about their children's identity studies was high and we experienced higher levels of parent engagement with documentation of identities. A future goal for our teaching team is to record 
children's reflections and conversations during ongoing collaborations in identity studies.

As I come to an end of my prologue, I note how in our school we transitioned in our teaching from individualists to more collectivists in our approach to identity studies with young children. Chang (2008) stresses, "The concept of culture fundamentally affects how we conduct a cultural study; it shapes our research questions, our sources of data, our analysis/interpretation, and our writing" (p. 15). I agree with the several cultural notions raised by Chang (2008): culture is inherently collectivistic, not individualistic, and at the same time individual autonomy is the foundation of innergroup diversity. Now, it is important to turn to the bigger problems facing identity as a social construction in schools for young children.

\section{Purpose of the Study}

The purpose of this research study is to conduct a narrative inquiry into Reggioinspired early childhood educator's experiences of co-constructing social identities with children through reuse materials. This dissertation seeks to illuminate that in Reggioinspired educators' social constructivist experiences, pre-primary age children are capable of co-constructing complex knowledge (Duhn, 2011; Lesnik-Oberstein, 1994) and representing their theories on identity and ecology through reuse materials (Reggio Children, 2005).

Critical engagement with such complex issues as identity and ecology can generate space for new understandings of how early childhood education can contribute to the theory and practice of identity studies and education for sustainability. There are 
many inspired ways to foster young children's learning and capabilities in responding to cultural and ecological issues through education for the environment such as reduce, reuse, rot, and recycling (Davis, 2009; Mackey, 2012;). As a local and global ecological culture, we are grappling with the ecological effects of organic and non-organic materials management and adult education has been the primary tool for fostering a culture of recycling. When young children are included in making decisions that impact their lives, including decisions regarding ecological sustainability, they are capable of contributing to conversations as competent citizens that lead to purposeful action in caring for their environment (Mackey, 2012). In order to decrease the generation of waste materials, it is helpful for children, families, and educators to understand what waste materials are, where they come from, and where they go.

The dissertation seeks to document the new and evolving epistemological spaces created by Reggio-inspired educators who socially-construct identities with children using reclaimed materials. Innovative pedagogies discussed in this research proposal examine the right of materials to become something else through educational reuse, and the right of children to work with complex ideas and materials in pre-primary schools. Innovative theories of social and ecological learning discussed and explored in this paper challenge traditional developmental theories about children's identities. These challenges are based on early childhood identity studies in social constructivist settings that document preprimary age children's ability to work with complex moral and democratic issues across a range of contexts within their everyday social interactions (Johansson, 2009). 


\section{Evidence and Significance of the Problem}

This study is significant because the preprimary school years are a crucial time for identity and language (Copple \& Bredekamp, 2009) as well as for education for sustainable consumption, reuse, and recycling. This research study is focused on the gap in research on social and ecological identity construction as dimensions of children's selfawareness in early childhood educational settings. According to Horton, Hadfield-Hill, Christensen and Kraftl (2013), "Childhood and youth are typically crucial periods in the socialization and (re)production of individual habits, norms, dispositions, values, lifestyles, identities, and feelings of care and belonging which can have all manner of complex, enduring environmental, ecological and political consequences" (p. 250). The importance of engaging young children in environmental learning has been recognized in education as a key element in cultivating a potentially life-long disposition of care for the environment (Barratt, Barratt Hacking, \& Pat, 2014; Barrat Hacking, Barrett, \& Scott, 2007; Chawla, 2007, 2009; Davis, 2009; Hicks \& Holden, 2007; Parnell, Downs, \& Cullen, 2017; Reggio Children, 2005). According to a 2010 study, $66 \%$ of children 3 to 5 years of age in the United States participate in childcare and preprimary school services. (www.oecd.org/social/family/database). Early childhood education is an important part of Brofenbrenner's (1979) concept of mesosystem in human ecology where such a disposition can be fostered as early as possible in a child's life. However, when it comes to engaging young children critically with complex ecological issues, there is a paucity of research, methods, and curriculum and instruction resources to guide the socially constructivist early childhood educator. 


\section{Initial Statements on Research Methods}

I employed narrative inquiry methods (dyad interviews, a focus group interview, and artifacts to be explored in depth in Chapter 3) to describe and explore how Reggioinspired early childhood educators socially construct identities with children with reuse materials. This study creates a space for the voices of early childhood educators who use alternative and reclaimed materials. I interviewed 6 educators in dyads, who identify as Reggio-inspired social constructivists, about their identities as educators, and their experiences designing constructivist curriculum and instruction connecting children's ecological identity to reuse materials. These paired inquiries zoomed in on early childhood educators and how they define and describe the experience of collecting, organizing and exhibiting repurposed materials to bridge identity and reuse materials as well as their pedagogical documentation of making identities visible.

The narrative data collected through dyadic interviews is metaphorically interpreted using a reflective process. The participants are educators who identify as social constructivists and work in a Reggio-inspired preprimary school setting. The intention of the study is to delve deeply into the perspective of these early childhood educators about their experiences with identity studies using Remida-inspired materials.

\section{Research Question}

This paper examines, through a narrative methodology, the following research question: What are early childhood educator's experiences with reuse materials in Reggio Emilia inspired identity studies? 


\section{Chapter 2: Review of Literature}

How we construct our views of identity shapes our view of teaching and learning. In this chapter, I elucidate some key intersections between the literature of Chicana epistemology and liberation praxis, postpositivist realism, situated learning, and social constructivism and describe how these lenses were applied in this study to examine Reggio-inspired educators' experiences of socially constructing identity with 2- to 5year-old children using Remida-inspired reuse materials.

First, I introduce the context of this study and theories that influence pedagogical practices found within Remida creative recycling centers. This context is followed by a description of the Remida-inspired materials to enhance a clear understanding of the physical stimuli involved in this study that examined six Reggio-inspired educators' perspectives. This inquiry can work to broaden educators' knowledge of the diverse ways that social membership informs children's theories and work as well as raise awareness about the range of perspectives and cultural interpretations that children's identities hold. This review of literature also involves an examination of close ties between culture and issues of learning to better understand the intersections of individual and social identity factors in the context of Reggio inspired early childhood education for sustainability (ECEFS).

With one recent notable exception (Parnell et al., 2017), I found no empirical studies specifically involving interviews with Reggio-inspired educators who coconstruct social identity with young children using Remida-inspired materials. The small size of the body of literature regarding Remida-inspired experiences in early childhood 
education underscores the need for the study. However, to promote an understanding of the nature of these Reggio educators and their work, this literature review presents the broader research regarding identity studies in early childhood education and key theoretical perspectives that informed my research question. Specifically, this study's research question is: What are early childhood educators' experiences with reuse materials in Reggio Emilia inspired identity studies?

I will discuss historical ways of thinking about young children's developmental progressions followed by an examination of more recent research. Here, I will examine the Borderlands and Reggio-inspired circular perspectives and how they apply to early childhood educators' experiences with socially constructing children's identities. I also consider which aspects of situated learning and liberation praxis assist in theorizing the relationship between identity and reuse materials for early childhood educators.

First, I share a brief history of waste and materials management, which is needed to highlight both the historical context of discards and the value of Remida-inspired early childhood education for sustainability.

\section{A Brief History of Materials Management}

It is helpful to examine how Western thought has evolved about waste and its treatment over the millennia. According to Ezban (2012), unlike modern waste materials, the history of discards began with one single organic waste stream. Ancient societies of hunter-gatherers were nomadic people, constantly moving to where food was available. Their waste consisted almost entirely of organic material—derived from plants and 
animals - that they discarded as they moved through an area. It was only as populations became settled that solid waste management became a greater problem.

When resources were depleted and the heaps of discarded material became an issue, people simply moved (Ezban, 2012). Gradually, populations became more concentrated in towns and cities where a solid waste crisis began. For millennia, humans also used organic waste as compost for crops because it proved to be highly useful and valuable nutrients. As cities grew larger and became increasingly distant from their food sources organic waste became viewed as something that was no longer useful, it became garbage. The garbage heaps grew as populations increased, but communities could no longer pack up and move to escape their discards. The municipal dump was born around 500 B.C., when Athens issued the first-known law against throwing garbage into the streets. Instead, the law required residents to dump waste no less than one mile outside the city walls. As urban areas grew and the space to discard trash became scarce, dumps were centralized and systematized, evolving into what we today know as sanitary landfills (Ezban, 2012).

Here we see the historical shift from producing mostly organic waste to a large percentage of materials being produced that are not biodegradable or viewed as reuseable. Also, it is important to note that Western culture developed a "throw away" mentality that is not apparent in every culture around the world (Ezban, 2012). That part of the dis-ease of Western culture is this sense of disconnection between our self-concept and the materials we interact with and how we value or fail to value them. This disconnection is captured in the suggestion "We are what we throw away." Rathje and 
Murphy's (2001) research on the archeology of garbage resulted in their conclusion that what people have owned and thrown away can express more about the lives they lead than they themselves ever may.

Contemporarily, in the U.S., the Environmental Protection Agency (EPA), has conducted various government, industry, and university surveys to track annual municipal waste and materials generation. Each year, the EPA produces a report called Advancing Sustainable Materials Management: Fact Sheet, formerly called Municipal Solid Waste in the United States: Facts and Figures. This national research has shown that due to hyper-consumerism, North Americans produce more trash than any other country, throwing away about 7.1 pounds per person per day, 365 days a year (epa.gov, 2015). Further, Humes (2013) informed us that the EPA estimates that, between 1980 and 2000, the average American's daily trash load increased by a third. In 2014, Americans generated about 258 million tons of trash and recycled and composted about 89 million tons of this material, equivalent to a $34.6 \%$ recycling rate.

A significant contribution to this problem is the throw-away mentality that has grown significantly since many store-bought materials come packaged in instant garbage (the boxes, wrappers, bags, ties, bottles, caps, and bubble wrap that contain products). According to Humes (2012),

In many cases, what is inside that packaging is destined to break, become obsolete, get used up or become unfashionable in a few years, months or even days - in other words a significant percentage of what Americans buy rapidly become landfill material too (p. 5). 
Many cities in the United States have put policies in motion that attempt to make their cities zero waste. One of the aspects of these zero waste plans is to increase recycling and composting rates.

To summarize, humans once produced only organic waste that was first viewed as something to leave behind or put aside, then as something useful for growing new food, and then increasingly as something to be put aside. As a result of this mentality, that waste is something to be put aside and out of mind, Western culture increasingly produced materials intended to have a short shelf life. Particularly those living in urban and then suburban settings let go of the idea that waste could be used to create something new. Instead, to many people, our material discards became less a part of formation of social identity offering connectedness and wholeness within a cycle (recycling for a greater purpose), as the garbage piled up around them becoming ever-present and seemingly useless. Alternatively, new waste cultures appear to be springing up presently.

\section{Remida Reggio Center for Creative Recycling}

Centers like Remida — a cultural project that emerged in Reggio Emilia, Italy_co-construct social identities in infant/toddler centers and preprimary schools through giving value to waste materials. Remida centers (over 17 worldwide) represent a sensitive, relevant approach that respects a child's understanding of intelligent moderation and allows them to have a voice (Reggio Children, 2005).

The Remida creative recycling center opened in 1996 and was run by the Municipality of Reggio Emilia and AGAC, the company supplying gas and water to the city (Hobba, 2006). The original Remida Center, 
Represents a new, optimistic and proactive way of approaching environmentalism and building change through giving value to reject materials, imperfect products, and otherwise worthless objects, to foster new opportunities for communication and creativity in a perspective of respect for objects, the environment, and human beings (Reggio Children, 2019).

Barratt Hacking, Barratt, and Scott (2007) drew attention to the importance of policy that acknowledges children's involvement in democratic decision-making as a way to engage them in ecological issues. In keeping with this perspective, the Remida creative recycling center holds a vital place in the living system (Cadwell, Ryan, \& Shwall, 2005) of Reggio Emilia, Italy and increasingly so in international Reggio-inspired educational systems.

In Reggio Emilia, Remida is run by the association "Friends of Reggio Children" and is a vital part of the Reggio Emilia educational system, in which adults and children learn in a complex web of interdependencies. These interdependencies call to mind Brofenbrenner's (1979) bio-ecological systems theory of human development.

In Brofenbrenner's (1979) model, there are five environmental systems identified with which a child interacts; microsystem, mesosystem, exosystem, macrosystem, and chronosystem. For this study, the microsystem and mesosystems are the two levels that I focused on for this study and an explanation of these levels relative to Reggio are to follow. The microsystem refers to the institutions and groups that most immediately and directly impact the child's growth including family, school, religious institutions, neighborhood, peers, and the child's own biology. I view the microsystem construct as 
highly relevant to my research study because it provides a framework from which to study the relationships of children's contexts within communities and the wider society. Per this philosophy, each system contains roles, norms, and rules that may shape human psychological growth and change.

Brenfenbrenner's mesosystem is also highly relevant in this study. The mesosystem is the interconnection between the microsystems, such as interactions between the family and educators and relationships between the child's peers and his family. This human ecology framework facilitates organizing information about people and their environment in order to understand their interconnectedness.

In the Reggio Emilia educational system, the infant-toddler or preprimary school teacher always works with a co-teacher. Together, these two classroom teachers relate to the other teachers, auxiliary staff, and the studio teacher in their school as well as receive support from a pedagogical coordinator, mentor teachers, cultural mediators, staff assigned to the Documentation and Educational Research Center, Remida (the Recycling Center), and other laboratories and resource centers found within the school. Educators also interact with parents who are involved in the life of the school (Edwards et al., 2012). This web of connections is very much in alignment with Bronfenbrenner's mesosystem model.

The Remida Center has been created to support a very different image of children than some contemporary theories of child development (Burman 1994). Their image is of children who are active protagonists or equal participants (adult and child) in the education process. 
Founder of the Reggio Emilio approach Loris Malaguzzi (1993) spoke of the capable child born with a hundred languages as "strong, powerful, competent and most of all connected to adults and other children" (p. 10). Children are citizens with rights and are co-constructors of knowledge and identity. Within this context, recycled materials offer a wide range of possibilities including provocations for debate, languages of expression, tools for thinking, sources of beauty, stimulus for design (Hobba, 2006) and a means of connecting to reuse materials and gaining a deeper understanding of each other's identity and social identity through reuse materials.

Remida-inspired approaches to reuse offer pedagogy of hope; the strength of the experience is that it creates a possibility for children to understand an authentic value of planet caring. It does this through the creative process (Parnell et al., 2017). The term pedagogy means theories of teaching and learning and how we enact those theories. The Remida Center offers an opportunity to translate a sense of social and environmental responsibility into concrete actions. In addition to pedagogy (child-lead instructional focus), Remida offers an andragogy (Knowles, 1980) of hope. Remida is a good example of how to shift early education to support the facilitation of children's ecological identity. To do this, educators must learn to think and teach differently. I interviewed adult educators and examined the formation of their social identities as Reggio inspired educators rather than interviewing children or children and adult educators in this study. Remida experience highlights remainders, a world of elements and traces, which, upon leaving industrial production, may no longer have value, but can receive a new life at Remida in other forms. The materials take on new and diversified identities. The 
Remida project, a growing worldwide phenomenon, “...gives attention to the search for new sensory grammars also in relation to new generation materials and elicits curiosity toward the entire production and distribution process." This philosophy stimulates a “commitment toward designing materials and objects whose recycling and reuse are already contemplated" (Reggio Children, 2019, para.7).

Knowing and living in this Remida-inspired project, I claim that lacking an epistemology of ecological identity, early childhood educators will be left with few options for critically approaching and perhaps reversing the political and economic processes that perpetuate unsustainable consumerism in succeeding generations. Remida gives us the hope and forward-thinking movement needed to change course in today's era of hyper-consumption.

\section{Remida-inspired reuse materials.}

Giacopini (2009) evoked a strong message around Remida-inspired reuse materials worth reflecting on and sharing, "Objects can not only invoke in us our own experiences; they can also suggest to us a memory of what the particular object could be" (as cited in Montoya, 2009, p. 10). Reflecting on alternative, reclaimed, reuse, salvage, and other unfinished materials found in schools today has become increasingly central because of their connection with the themes of ecological sustainability, contemporary thinking, and pedagogical potential. Exploratory studies conducted by Guerra and Zuccoli $(2012 ; 2013)$ confirm that working with discard materials is useful for early childhood education in that it helps develop strategies for experimenting, broadening, and deepening the use of materials at school both theoretically and methodologically. The 
studies also show that the choice of materials to use is more and more intentional on the part of early childhood educators.

The following literature review of Remida-inspired reuse materials education from 2009-2014 spans exploratory surveys, reflections, and position papers on art education, case studies, and action research. This preliminary survey of the literature focuses on the questions and thoughts that arise from educators and researchers who coresearch Remida materials with young children and the conceptualizations they have developed from grasping the processes and the potential stemming from developing relationships with discard objects and materials in early childhood education.

Guerra and Zuccoli (2012) conducted an exploratory theoretical and empirical survey that investigated the meaning and effect of working with materials in preschools. They discovered that,

The indefinite qualities of unstructured materials, especially materials discarded from industrial processes, offered new opportunities both for the teachers leading the workshops, as well as creating very specific areas of investigation for the children who experimented with several languages and thus learned on different levels, stimulating them to find creative strategies to deal with the questions that came up. (p. 726)

Guerra and Zuccoli (2012) introduced their studies noting that the importance of materials and objects has historically been part of the educational philosophy of many educators. They asserted that Froebel (1826) explored the objects collected by children and the relationships with these objects, which, define the child's very essence, creating 
the boundaries and giving the child an idea of him or herself. Furthermore, Edwards, Forman, and Gandini (1998) placed Remida Centers within a global ecological context, The proposals, with differences and specificities, dedicated to bringing discarded and recycled materials into schools are present throughout the world. A bastion of working with discarded industrial materials in schools is Remida Reggio Center, part of the Reggio Emilia Approach, deeply interested in the connection between children and their environment. (1998, p. 170)

A survey conducted by Guerra and Zuccoli (2012) confirms that working with unfinished materials, as shared in the Remida project, aids children in using creative thought, "meaning their capacity to generate new, original connections between information, thoughts, and objects. The juxtapositions and the transformations of the objects allow new, meaningful and valuable attributes to be made, encouraging new and original viewpoints regarding everyday life" (p. 726). Their study, like all the others mentioned above, highlights the fact that this approach is not brand new and has been shown to have benefits to children.

Tarr's $(2001 ; 2003 ; 2008)$ reflections, deeply inspired by her study tours to Reggio Emilia, are framed in a manner intended to promote open conversations with art educators based on the image of the child as creator of culture, the "environment as the third teacher" (Gandini, 1998, p. 177) and aesthetic codes in early childhood classrooms. Regarding Remida materials, she cited the metaphor of materials as language and the power of listening. Tarr highlighted the interdependency of form and function and encourages, "Using materials as languages — not just for aesthetic purposes through 
creating beautiful images and objects, but also for making thinking processes visible to peers, teachers, and parents for understanding and communication” (2008, p. 20).

Similarly, Gandini and Kaminsky (2005) recalled that the Remida coordinator, Graziella Brighenti, extended the notion of listening to include reuse materials. "That materials have a right to be listened to and understood, that they show traces of their past identity, and that materials have the power to invoke in us our own experiences" (Gandini et, al). Brighenti contests narrow images of the child and cites "Impressive examples of young children (including infants and toddlers) exploring materials that go far beyond what most teachers considered... suggest[ing] many possibilities for using found, recycled, or natural materials as part of areas of study across curriculum” (p. 24). Further, Tarr (2003) described the many natural and recycled manufactured Remida materials in Reggio Emilia classrooms and how the use of materials is deeply embedded in and integral to the life and culture of the classrooms. She explained how they are displayed in clear containers to enhance their visibility and by color or in a way to highlight their aesthetic qualities, "In the preprimary schools for children from 3-6 years, I have seen children's work in weaving, stitchery, and constructions with chicken wire and recycled materials; detailed and elaborate projects involving multiple media including technology, explored from multiple points of view" (p. 8).

The intention with which each material is considered for its potential to become a language for expression is also evident in Montoya's (2009) account of the reuse materials at the Wemagination Center, part of the University of New Mexico's Family Development Program. "The materials have their own stories to tell and invite visitors to 
investigate them...Every material placement is intentionally thought through. It all has a purpose" (p. 10).

Foreman (1994) used the term affordances to describe how each medium provokes a special orientation to the problem to be solved and that children learn to make compromises with what the medium does not easily afford, "The environment and the materials invite visitors to go on a journey of creative possibilities" (p. 9). Montoya (2009) compared Wemagination Center to the Hive Project (Kelly \& Lukaart, 2005) and Remida Center, “All three have a deep respect for materials by promoting them as useful and beautiful beyond what is normally recognized" (p. 9).

Like many Reggio-inspired educators, Wexler (2004) reflects on the principles of emergent curriculum, the image of the child, project work, and listening; "Because listening means to deeply engage with the world, an important gift to give children is time to make listening possible" (p. 17). She observed "Their materials are not glamorous, slick, or 'made for children' - the kind we see in teacher catalogues. They use humble materials, such as screws, plastic, bottles, glass, cardboard, and wire, which have been brought from Remida, a local recycling center" (p. 16). Additionally, Montoya notes,

There is a circularity in their work with materials that reflects the way the children develop ideas. Learning about one aspect of the material usually leads to a new discovery, and so the materials are revisited as new ideas create new ways of working (p. 16). 
Reuse self-portrait work is an example of a project children make with discard materials and then discuss what they represent — what is there, what is missing, what is accurately depicted, and what is either vague, or somehow off-to surface children's self-concept through discussion and help them to see themselves personally and in relationship with others. This is the nature of the listening that Montoya is referring to and why it is a gift to children.

Rinaldi (2006) coined the term intelligent materials referring to the languages of discard materials and the educational value of infusing Reggio Emilia principles into creative reuse education in early childhood. For example, the term affordance is used to describe how each medium including complex reuse materials, provokes a special orientation to the problem to be solved and thus assuming a critical role in the concept of the Remida-inspired environment as the third teacher. This conceptualization is further elaborated in a publication entitled Reggio Children (1998):

The concepts of interaction and interface suggest a parallel between interpersonal relations (based on dialogue) and the relation between people and the objectworld (also based, if you will, on dialogue, comprehension and identity). Objects, which have always spoken to us through their form and social role, now dialogue by exchanging information in an increasingly sophisticated language, which includes an almost complete sensory involvement. (p. 14) Fyfe (2012) specifically mentions how self-portrait work is used to facilitate children's social identity formation in a story about the relationship between listening, documentation, and assessment. Educators at an urban Reggio-inspired preschool in 
Missouri were becoming increasingly aware of families growing interest in a self-portrait study that was made visible through documentation in the school hallways. For fall conferences teacher educators decided to use the self-representations as a focus for dialogue. Fyfe (2012) notes, "Describing the process of the self-portraits provided an insight for parents regarding the relationships being built between the children and teachers as they collaborated on this meaningful work. The developing observational, expressive, and reflective skills of the children especially came to light” (p. 288). In addition to self-portraiture work with small groups of young children (Gandini, 1998; Kaminski \& Gandini, 2002), this instructional approach has worked with mixed groups.

As noted in the key terms in Chapter I, the definition of social identity is a key concept with emerging literature on family identity in early childhood education highlighting how a family is the most basic element of self-awareness for young children; it shapes and informs their sense of self and who they are in the world. In this regard, gaining insight into the identities of children within the context of their families through parent participation in early childhood identity studies is invaluable. Additionally, collaboration amongst educators, children, and families is a reoccurring theme in the early childhood for sustainability literature as a means for children to experience the power of community to bring about sustainable changes.

The limited body of research on children's three-dimensional art (Golomb, 2002; Golomb \& McKormick, 1995; Pavlou, 2009) indicates that "young learners readily engage in such work and attend to the representational issues inherent in threedimensional art, such as form, uprightness, balance, stability, complexity, and 
movement" (Eckhoff, Hallenbeck, \& Spearman, 2011,p. 41). For example, as part of an action research study, Helm and Parnell (2010) facilitated collaboration among preschool children, teacher educators, and parents. This collaboration was designed to observe the effects on children's learning in a child development re-use center, "A space available to anyone at the school where various open-ended salvage materials (i.e.: milk caps, yarn, cardboard pieces, plastics and more) are displayed on shelves and made available to children" (p .2). Helm and Parnell (2010) observed,

Working with parents also provided insight into the identity of the children within the context of their families... Taking cues from the children about materials and themes for exploration was a metaphorical dialogue, which also enhanced the relevance of the project to their natural learning paths, allowing us to more closely examine reading, writing, reusing, and social development (p. 12).

Helm and Parnell (2010) expressed the metaphor of transformation that provided clues to thinking about social identity in their research. The authors noted the children, Spent most of their time in the Re-Use Center engaged in this type of activity... they often mixed potions in order to trap their small robots. We heard words like 'float' and 'dissolve' as the children transformed water into bubbles, one color into another, and trash into treasures as Topal and Gandini (1999) suggested (p.11).

Reggio Emilia's perspective on children's communicative literacy is articulated well by Abramson (2008) in her reference to Eco's (1976) semiotic theory as an alternative explanation. She cited Eco's (1976) conceptualization of signs and sign functions as a 
generator of meaning; the sign (a symbol for meaning) is the basis for language and is the constructive element of all communication systems. According to Eco (1976), the sign represents a primary unit of culture, "That system of shared and interconnected meanings that have been organized over time into codes (language, gesture, music, etc.). Communication is by definition an interactive process that involves interchange with people, the environment, culture and symbolic systems" (Danesi, 2000, p. 136). When educational experiences are grounded upon semiotic theory, learners have opportunities to engage "a hundred languages" including the languages of reuse and identity, to communicate their ideas.

From a dialectical materialist epistemological view of consciousness, human interaction, and material transformation, Freire (1982) wrote, "Language is not only a medium for conveying new ideas; it is also a means whereby new meanings are constructed" (p. 76). In his view, words are more than instruments making dialogue possible - they also contain the two dimensions of reflection and action.

To use a new word is to transform one's world. For Freire (1970), “To exist humanly is to name the world, to change it. Once named, the world in its turn reappears to the namers as a problem and requires of them a new naming" (p. 76). As Anzaldua (1987) declared,

I am cultured because I am participating in the creation of yet another culture, a new story to explain the world and our participation in it, a new value system with images and symbols that connect us to each other and to the planet (p. 1). 
Further, Shapiro (1994) observed, “Although scientists frequently create a new language to re-conceptualize and rename the words, rarely are students shown the creative nature of this process" (p. 33). Semiotic theory reminds us that there are diverse languages for communication. As well, according to Malaguzzi (1996), children have a hundred languages for expressing their cultural theories; the hundred languages as a metaphor for semiotics. Thus, communication is an interactive process that involves exchanges between people, the environment, culture, and symbolic systems (Abramson, 2004). As such, semiotic mediation is integral to social constructivism, Chicanx studies, and Reggio-inspired early childhood education.

Mooney (2013) observed that “today's Reggio-inspired educators also believe that what children learn from their peers and from the materials in the classroom is as important as what they learn from their teachers" (p. 101). Similarly, Wexler (2004) spoke to this idea of how representational media such as reuse materials are used in the Reggio Emilia schools to engage and deepen children's understanding of a theme or concept. Such materials also engage children as much as they do educators, too. Helm and Parnell (2010) provide a deeper explanation of the Environment as Third Teacher concept for their Remida-inspired research citing Ceppi and Zini’s (1998) description of environments, which suggested, "using the following keywords: overall softness, relation, osmosis, multisensoriality, epigenesis, community, constructiveness, narration and rich normality. Their seven environmental tools include relational forms, light, color, materials, smell, sound, and microclimate" (p. 3). Expressive workshops of joint compositions of colored forms documented in Children, Art, Artists (Reggio Children, 
2005) relates the multidimensional nature of art therapy for the purposes of evolving selfconcept and social identity.

During expressive workshops in Children, Art, Artists (Reggio Children, 2005), individually produced oil pastels were place on a large sheet to make up a single large joint composition. The authors cite a child who exclaimed: "when we joined our colored forms together we got the feeling of an explosion!"” (p. 124). Educators addressed both ideas (self-concept and social identity) and the interrelated nature of these constructs by introducing an element of variation through the suggestion that children should use their individual works to produce on large joint composition. To better understand the contemporary perspective of identities studies presented here, it is helpful to understand the historical development of the constructs explored in the next section.

Reggio founder Malaguzzi expressed his belief that new languages will emerge from Reggio- inspired “ateliers” or studios and laboratories. Malaguzzi’s (1920-1994) poem entitled No Way. The Hundred is There refers to symbolic languages, including drawing, sculpting, dramatic play, writing, and painting, and storytelling used to represent children's thinking processes and theories. In this poem, he envisioned new languages that would emerge from the studios and laboratories of the Municipal PrePrimary Schools of Reggio Emilia. A line in the poem reads "The child has a hundred languages (and a hundred, hundred, hundred more)" (Edwards et al., 2012, p. 3). Indeed, the new languages of the digital and reuse emerged from the studios (Remida Series, 2016), as Malaguzzi predicted. 


\section{Meta-Frameworks}

A theoretical perspective is the point of view or lens through which research is conducted and data is analyzed (Kilbourn, 2006). As an educator-researcher who values multiple perspectives, I appreciate the benefits of not restricting a study to any single theoretical approach. Therefore, my theoretical approach to studying ecology, culture, and learning in early childhood education is through a paradigm that involves multiple theoretical lenses. In this section, I will weave together a picture of how particular bodies of literature shaped how I conducted my research and interpreted my data. These bodies of literature are Chicana feminist frameworks, postpositivist realism, situated learning theory, and social constructivism, Borderlands epistemology, and liberation praxis.

\section{Chicana studies.}

As a social constructivist and Chicana studyist, and as a reflective researcher, I recognize that subjectivity shapes my theories. My interpretations flow from personal, cultural, and historical experiences. Chicana feminist studies emerged from the field of Chicanx studies. Several different frameworks emerged from the Chicano Civil Rights Movements (1965-1980) including deconstructionist interpretations of the experience of being an American of Mexican descent. Learning communities involved in Chicanx studies tend to heavily engage deconstructionist semiotic interpretations.

Deconstructionism, as aforementioned can be described as a theory of reading in adult education that which aims to undermine the opposition within texts.

Chicanx studies is a democratic approach to education that seeks to develop scholar-activists prepared to make well-informed policy decisions based on an awareness 
of the intersections of social, environmental and economic justice. The field fosters selfconscious researchers able to construct knowledge that is post-colonial in nature and grounded in critical pedagogy (McLaren \& Leonard, 1993).

In this study, I engaged Anzaldua's (1987) Borderlands epistemology of identity that suggests that in the straddling of two cultures, a hybrid or mestiza identity (a woman of mixed race, especially the offspring of a Spaniard and an American Indian) is created; an identity of resistance to hegemonic methodologies and pedagogies. The mestiza-a woman of mixed racial ancestry, especially of mixed European and Native American ancestry — is described as resilient by nature and therefore capable of embracing, practicing and becoming natural leaders in creating a new holistic world view. Anzaldua (1987) describes how by drawing on her indigenous heritage and personal experience of living in the Borderlands, the mestiza can accept her "interstitial material existence" (p. 9).

As a half Anglo, half Mexican, I identify emotionally, biologically, and intellectually with what Anzaldua described as a "new mestiza," a person of mixed ethnicities, aware of her incongruent and interlocking identities, and who consciously bridges them to challenge Eurocentric dualism. This hybrid identity formation is a phase she calls neplanta: An Uto-Aztecan term for the "transitional space a person occupies when experiencing transitions or the personal dichotomy that occurs between shifts of awareness" (p. 106).

My social identity formation of mixed European and Mexican Indian ancestry developed in my formative years growing up along the borders of Mexican, American 
and Southern European cultures. I have alternately identified as Mexican-American, Spanish, Chicana, White/Hispanic, and American, depending on my social or geographic context, or my consciousness at the time. My identities shape my theories and the multiethnic perspective taking that framed this study.

The frameworks that emerge from Chicana feminist epistemology in educational research can be quite different from the perspective of mainstream academics. New theories of knowledge allow Chicanx studyists to interrogate the social situation in a novel way (Delgado Bernal, 1998; Pizarro, 2004). For example, Anzaldua coined the term autobiographical theory or autohistoria-teoria, which signifies a transformation of traditional Western autobiographical forms informed by reflective self-awareness in the service of social justice work. Autohistoria focuses on the personal life story but, as the auto-historian tells her own story, she simultaneously tells the life stories of others (Keating, 2009). Key literature relates the use of self-portraits as a pedagogical tool in facilitating reflections on and formation of social identity (Kissinger, 2017; Edwards et al., 2012; Topal \& Gandini, 1999; Whitney, 1999).

Examples of how self-portraits are used to facilitate children's exploration of racial, ethnic, and cultural identity are highlighted in the work of Kissinger (2017) and Whitney (1999) and specifically framed around topics often addressed by the Borderlands perspective such as ideas to do with socio-economic class, national identity, and populations. Kissinger's (2017) anti-bias work in the early childhood classroom involves self-portraits as a strategy for keeping teaching practices in touch with growing social movements. Self-portraits are a tool for supporting children noticing different skin 
colors, which opens intentional conversations around differences in genders, religions, family structures, and other social identification.

Whitney's (1999) work focuses on the power of persona dolls as one of the most amazing anti-bias tools in the early childhood classroom. Persona dolls serve a different purpose from dramatic play dolls in a classroom. When children encounter a classroom persona doll they are interacting with another member in the classroom. To achieve this, a persona doll is created whose identities reflect characteristics of real children in the classroom and whose life experiences unfold just as they do for children in the classroom. These individual and social identities help the children connect with the dolls and make the stories of the dolls more powerful. This is unlike a dramatic play doll in the classroom whose age, name, family, identity, and gender can change any time a child in the classroom desires it.

Storytelling with persona dolls (dolls that are given names, family histories, and other traits by educators),

Is a powerful tool for teaching classroom and social skills, giving children words for and tools to manage their feelings, developing problem-solving and conflict resolution skills, expanding children's comfort with difference, undoing stereotypes and biased information, and helping children to stand up against bias ( Whitney, 1999, p. 233).

In my study, I found that inviting educators to share their own stories of working with self-portraits constructed with reused materials provides insights into how they simultaneously tell the life stories of others (Keating, 2009). 
The experience of multiple identities is a distinctly metaphysical one for Chicanas and the constant crossing between cultures becomes an ordinary occurrence (Rodriguez, 1994). A linguistic dimension of learning identity in the Borderlands often involves two ways of speaking, and sometimes distinct languages. Delgado-Bernal (1998) stated, "Current and future generations of Chicana scholar-activists continue to raise our voices . . as we (re)construct the ways we hear, interpret, and learn from and within a Chicana feminist epistemology in educational research" (p. 560). Chicanx studies learning, and teaching practices demonstrate how cultural productions are sites where epistemological forms of domination can be contested and recreated in ways that allow for the creation of alternative identities and subjectivities. I applied this lens in my examination of how alternative educators inspired by Remida perceive the formation of social identity and in terms of how they research with children.

\section{Postpositivist realist theory of identity.}

I embrace a postpositivist realist paradigm as articulated by Gilpin (2006) on the epistemic significance of identity. Gilpin finds that transformative understanding emerges from one's past and present experiences and interactions when interpreted in sociopolitical contexts. Understanding, then, is relative to one's experiences as raced, gendered, classed, nationalized, and so forth. Although multifaceted, she summarized the four tenets of postpositivist realist theory as: 1) Identities are both constructed and real; 2) Identities are mediated through cognitive and social processes; 3) Knowledge garnered in the context of oppression should be afforded epistemic privilege; and 4) The power of individual and collective agency should be part of discussions of identity (Gilpin, 2006). 
One could argue that postpositivist realist theory advocates for a Borderlands approach or a conceptual bridging of two seemingly incongruent paradigms. The bridging of positivist realism and postmodern reductionism transcends each of their ideological limitations. This inclusive theoretical perspective contests the dichotomization of positivist and constructivist paradigms.

Following the recognition that logical positivism was no longer tenable in modern science, post-positivism and its research methodology, critical multiplism, emerged and offered a new way of thinking about social science. Postpositivist critical realism recognizes that all observation is fallible, involves error and that all theory is revisable. Though not a cure-all, critical multiplism, in which methodological pluralism is advocated, offers a way around the debate of which conception of science is appropriate for education and, as such, poses advantages for early childhood knowledge construction (Letourneau \& Allen, 1998).

\section{Critical theory.}

My critical theoretical paradigm that involves multiple theoretical perspectives is deeply influenced by Freire (1970) and his concept of conscientizacion, or critical consciousness, that became popular among Chicano activists and educators in the 1970s. Critical Theory provides a basis for investigating "power relationships and the marginalization of some social groups and as it relates to education for sustainability, marginalized groups include children and future generations as well as non-human species, places, and even natural elements, such as water, soil and air" (Elliot \& Davis, 2009, p. 5). 
Critical Theory also helps in understanding how educational systems have had a part in marginalization. My transformative approach to education is inspired by Freire's liberation praxis which is grounded in post-Marxist critical theory. He believed that students must be directly engaged in their education as a revolutionary act in a quest for mutual humanization. Mutual humanization can be defined as "a profound commitment to fight social injustices in our struggle to recapture the loss of our dignity as human beings" (Freire \& Macedo, 1998, p. 56).

For Freire, critical consciousness materializes with constant and optimistic inquiry, experimentation and dialogue between student and educator. Most importantly, throughout his career, Freire encouraged students and educators to nurture a profound trust in each other as investigative partners and collaborators.

In Pedagogy of the Indignation, Freire was developing an eco-pedagogy. Here, Freire engaged critical class consciousness in his exploration of the relationship between what he referred to as the colonizer and the colonized. Freire theorized the individual under the capitalist systems acts with a false consciousness that does not allow them to recognize the very source of their oppression, therefore, individuals passively accept domination, not believing that agency and change are possible.

Since Freire's death, the Eco-pedagogy movement, influenced by his liberation praxis, has grown and further developed around the world. Liberation praxis proposes a novel relationship between educators, students, and society that is reflected in the notion of educators and children as co-researchers and co-learners (Rinaldi, 2006) as is found within the Reggio Emilia approach to early childhood education. 
This dissertation aligns with Grieshaber and Cannella (2001) as they object to the "imposition of colonization and colonizing constructs (i.e., individualism, minority, research) and to the constituting of identities through these constructs in education" (p. 18). The ideas of novel relationships and co-researchers are important to this study because, as objects of research practices, children have been marginalized and disqualified from having voices, as they are rarely consulted about their participation in research projects. Grieshaber and Cannella (2001) cite neocolonial perspectives of early childhood researchers who share the belief that the construct of the "universal child" is colonizing and that qualitative studies with children can be "hierarchical, undemocratic, creating power for the researcher, and embedded with voyeuristic, truth-oriented assumptions" (p. 19).

An antidote to "the continued effects of colonizing through discursive practices and philosophical domination" (p. 19) is embedded in such enduring questions as: "How does one socially construct a new kind of research with children that reflects their perspectives?” (p. 19). Reggio-inspired re-conceptualizations of children and educators as co-researchers shape my research and illustrate how Reggio-inspired educators make children's social constructions of identities visible through documentation. This study does not involve children's voices but only those of adults who research with children. This study addresses a gap in the literature by contributing foundation knowledge of educators' perspectives around how they construct knowledge with children through identity studies. This first layer of research will be useful for future research on ecological identity formation using Remida approaches. 


\section{Situated learning.}

Situated learning theory also informs this inquiry. Lave's (1996) theoretical work in situated knowledge and learning is aligned with Chicanx studies, social constructivist and Freirian frameworks of viewing learners as co-creators of knowledge. She writes,

It seems imperative to explore ways of understanding learning that do not naturalize and underwrite divisions of social inequality in our society. A reconsideration of learning as social, collective, rather than individual, psychological phenomenon offers the only way beyond the current state of affairs that I can envision at the present time (p. 149).

Chicanx studies value civic engagement and leadership skills and integrate service learning into student learning outcomes to help students understand how to bridge academic content and practice. Service learning is applied situated learning theory to foster an understanding of social justice principles and how to apply them to make a difference in students local, national and global communities.

My commitment to social justice combined with my belief in the idea that schools are institutions responsible for socializing the next generation (McLaren, 2014) draws me to situated learning theory. I was fortunate to experience a situated learning experience that inspired this study through a cognitive apprenticeship at Inventing Remida Project (IRPP), which is housed on a university campus in the Reggio-inspired laboratory child development center (CDC). A laboratory CDC is a school operated in association with a university, college, or other teacher education institution and used for the professional formation of future teachers, educational experimentation, and educational research. The 
early childhood master's program, the laboratory CDC, and the community are undertaking a cultural project with the aims of reclaiming and repurposing industrial discards and educating community members (especially early childhood teachers) on how to reuse these invaluable materials in the classroom, in school, and with community projects. The principles of Remida creative reuse are situated in the everyday activities that I took part in preparing and participating in the recycling center as a coordinator. At the center, we often say to newcomers, "You learn Remida by doing it!"

Brown, Collins, and Duguid (1989) described an approach that embeds "learning in activity and making deliberate use of the social and physical context [which] are more in line with the understanding of learning and cognition that is emerging from research" (p. 32). Brown, et al. cited Lave's groundbreaking work on learning, apprenticeship, and everyday cognition. Lave (1996) stated in her research on apprenticeships and informal learning, "Schools institutionalize, and are predicated on, widespread beliefs about learning that are called into question by views of learning as a situated activity. It suggests that new research questions are in order, about how learning in practice is characteristic of schooling" (p. 155). She encouraged new research paradigms aligned with informal learning pedagogies that "ask new questions about learning" (p. 155).

These ideas closely relate to this research study because in Reggio Emilia, both teacher education and children's learning are situated in practice, are collective and involve an immersed enculturation into the life of the classroom, school, and community. Situated learning examples are grounded in Remida-inspired action research (Helm \& Parnell, 2010) findings that reveal stories of language, literacy, and social constructions. 
Conclusions reveal that big questions for teachers, rich dialogue in reuse contexts for learning, and flexibility with participants are essential to collaborative learning in the reuse center.

Situated learning theory challenges abstract, decontextualized formal concepts and how "from the point of view of the dualist formal/informal model, indeed cognitive theory in general, culture becomes shared via cultural transmission. It is the transmitter's point of view that is implicitly privileged" (Lave, 1996, p. 154). To contrast old and new ways of teaching, in the Reggio examples I just provided, the educator and the children working as co-investigators are both in the transmitter role rather than the more typical one-way transmission model from adult to child. From this point of view, Lave (1996) noted how our understanding of both learning and teaching are outdated and static, "Inviting new analysis, which in turn requires novel analytic units and new questions" (p. 154). Inspired by this view, the situated theoretical perspective assisted my process of recognizing genuine conversations around identity and ecology that invited children into inquiry as valued members of the learning community and the preparation of learning environments where children bring their ideas to be appreciated, challenged, and transformed within a respectful climate (Reinisch \& Parnell, 2006).

Situated learning theory is also congruent with constructivist theories that view learning as social advancement that involves language, real-world situations, interactions, and collaboration among learners. In considering the value of engaging with objects and materials, Dewey (1938) advocated situated approaches to learning, arguing that understanding typically occurs within the context of social experiences. Dewey's idea 
was that children must have experience in order to strengthen the creation of knowledge and not as a mere exercise. He stated,

It is not enough to insist on the necessity of experience, nor on activity as part of an experience. Everything depends on the quality of the experience... Thus, the main problem of education based on experience is to choose the type of experience the children can re-experience fruitfully and creatively in the experiences that follow (p. 15-16).

This dissertation is bounded by the concepts of social constructivism, identity and sustainability studies in early childhood education and Reggio-inspired pedagogy. In discussing the range, complexity and symbolic force of constructivist ideas, Phillips (1995) noted, "Thus, in sum, human knowledge — whether it be the bodies of public knowledge known as the various disciplines, or the cognitive structures of individual knowers or learners - is constructed" (p. 5).

Phillips examined the range of social re-constructionists including the works of Dewey, Vygotsky, Piaget, and Kuhn and located their forms or sects of constructivism along several dimensions or axes. He asserted that constructivists such as Piaget and Vygotsky have been concerned with how the individual learner "goes about the construction of knowledge in his or her own cognitive apparatus; for other constructivists, however, the individual learner is of little interest, and what is the focus of concern is the construction of human knowledge in general" $(1995$, p. 7). Phillips added however, that along the continuum, there are many constructivists who have "an interest in both poles, and who believe that their theories throw light on both the question 
of how individuals build up bodies of knowledge and how human communities have constructed the public bodies of knowledge known as various disciplines" (p. 7). Like epistemologists von Glasersfeld (1984), Kant (1959), and Popper (1945), I have an interest in both poles. Regarding this study, I question how individual educators build up Remida-inspired concepts, terms, and activities and how learning communities structure knowledge to guide their Remida practices.

Radical constructivism stresses that knowledge construction is an individual matter and believes that individual's construct knowledge subjectively, filtered solely through the prism of individual biases, experiences, and sense perceptions (von Glasersfeld, 1984). Social constructivism pays more attention to the social processes in knowledge construction and emphasizes the role of culture and context in developing personal and shared interpretations of self-knowledge as cognitive constructs developed through relationships (Bruner, 1986; Piaget, 1970; Vygotsky, 1978). These opposing perspectives fit within the Chicanx studies perspective that heavily influences my paradigm. More specifically, the framework of Latino critical race theory (LatCRT) suggests that the social construction of race is central to how people of color are constrained in society. These tenets are what make LatCrt different because it looks at the differences between Chicanx students. These tenets are: the intercentricity of race and racism; the challenge of dominant Ideology; the commitment to social justice; the centrality of experience knowledge; and the interdisciplinary perspective.

Up to this point, I have highlighted the theorists and theoretical perspectives that shape how I conducted and analyzed my research: Chicana feminist frameworks; 
Borderland's epistemology; postpositivist realism; liberation praxis; situated learning theories; and social constructivism. Kuhn's (1962) work on the active role of scientific communities in knowledge-construction pointed to their major influence on constructivist schools of thought. He wrote, "The very existence of science depends upon vesting the power to choose between paradigms in the members of a special kind of community" ( $\mathrm{p}$. 166-167). In choosing a postpositivist realist paradigm, I maintain a non-dualistic Borderlands perspective of identity as both real and socially constructed. A Borderlands perspective creates a theoretical bridge that allows me to reconcile competing outlooks. This identity theory contests epistemological forms of domination in ways that allowed for my research subjectivity which bridged postpositivist and social constructivist theories of ecology, culture, and learning. The next section, which provides a review of literature related to early childhood education for sustainability, will further illustrate how this bridge functioned in this study.

\section{Early Childhood Education for Sustainability}

Many early childhood education for sustainability researchers highlight how for the past decade, education, including early childhood education, has been re-shaped by global discourses, institutions, and policies (i.e. Davis, 2009; Duhn, 2012; Duhn, Bachmann, \& Harris, 2010; Duhn \& Ritchie, 2014; Elliot \& Davis, 2009; Mackey, 2012). In 2002, the United Nations General Assembly (UNGA) adopted a resolution to put in place a United Nations Decade of Education for Sustainable Development (DESD), spanning from 2005 to 2014 . UNESCO was requested to lead the DESD and develop a draft of International Implementation (IIS). The IIS that resulted was cooperatively 
created by United Nations agencies, national governments, civil society organizations and Non-Governmental Organizations (NGOs), independent experts and specialists who propose that Education for Sustainable Development (ESD) is for everyone, at whatever stage of life they find themselves.

Therefore, ESD takes place within a perspective of lifelong learning, engaging all possible spaces of learning from early childhood to adult life. ESD calls for a reorientation of educational approaches - curriculum and content, pedagogy and examinations.

Agenda 21 is a comprehensive plan of action on sustainable development for the $21^{\text {st }}$ century that was drawn up after the United Nations Conference on Environment and Development (UNCED) held in Rio de Janeiro, Brazil, in 1992. It is intended to be applied globally, nationally and locally by organizations of the United Nations system, national governments, and groups in all areas whose human activities have an impact on the environment. It is a response to a call for documents and measures on how to create a more sustainable world made by the Brundtlandt Commission five years earlier (McKeown, 2000).

Agenda 21 was adopted by the 178 countries of the United Nations Rio Conference on Sustainable Development of 1992. Chapter 25 of Agenda 21 addresses children and youth in sustainable development as a major group. Children comprise nearly $30 \%$ of the world's population; the participation of today's youth in environment and development decision making and in the implementation of education for sustainable development (ESD) is critical to the long-term success of Agenda 21 (UNCED, 1992). 
Education has a critical role in aiding societies to make the transition to sustainable ways of living. All sectors - including early childhood education - must participate in re-imagining and transforming unsustainable patterns of living, but it is not likely that many early childhood educators have heard of these significant global initiatives (Elliot \& Davis, 2009). I am focused on social identity but am dedicating space to describe ideas related to sustainability here because social identity is foundational and inextricably linked to ecological identity.

Elliot and Davis, (2009) noted that the field of early childhood has been slow to engage with thinking and practice around sustainability issues, even though other sectors have evolved, and existing mobilization in early childhood education continues to be ad hoc. These scholars suggest the widespread belief among early childhood educators that traditional outdoor play in nature eliminates the need for environmental issues needs to be addressed and current early childhood education research is based in anthropocentric worldviews that blind researchers to environmental concerns. Further, the scholars note that the belief among educators that sustainability issues are conceptually beyond the grasp of young children, are too dire, and therefore inappropriate also blocks engagement with sustainability issues.

Early childhood education has been deeply shaped by wider social and cultural understandings of the child as vulnerable and innocent (Kincaid, 1992). There is a persistent idea of childhood as a time of innocence that should be kept free of complex knowledge (Lesnik-Oberstein, 1994). Culturally, childhood is often constructed as a time 
of innocence, which, can mean that complex issues such as ecological sustainability are considered too problematic for early childhood practice.

Ironically, for many years the same social constructs that link children to nature and innocence may be contributing to the paucity of research on early childhood education for sustainability and co-researching environmental issues with preprimary school-age children (Duhn, Bachmann, \& Harris, 2010; Louv, 2009). However, findings from a research project that focused on issues of ecological sustainability in early childhood centers in New Zealand from bicultural Western and Indigenous perspectives contribute a critical perspective of ecological sustainability as an educational issue in early childhood education (Rau \& Ritchie, 2014). The Whariki New Zealand curriculum for Early childhood education for sustainability (ECEFS) includes critical perspectives on social sustainability principles like well-being, belonging, and contributions to society. The curriculum also facilitates critical conversations around social and environmental sustainability with children.

Shielding children from complex ecological realities to guard their culturally constructed natural innocence (Ashenden, 2002) creates a serious challenge for educators when it comes to designing early childhood education philosophy, curriculum, and pedagogy. In fact, Duhn (2011) advised,

Making early childhood education more than a sheltered enclave that is dominated by romantic notions of childhood and nature requires imagination and courage, heart, body and mind. It demands discussion of the purpose, 
possibilities, and intent of early childhood education for society, and this discussion has to include children" (p. 27).

Critical engagement with such complex global issues as ecological sustainability generates space for new understandings of how early childhood education can contribute to the theory and practice of education for sustainability. Further, to the topic of Reggioinspired documentation in relation to social and ecological justice, Pelo (2006) suggested, "When we are genuinely engaged in pedagogical documentation, we will take up issues of social and environmental justice with children and families. The only way we can avoid these issues is by deliberately ignoring them when they arise" (p. 175). This documentation itself provides fertile ground for research into pedagogies related to social identity that lead to the formation of ecological identity. Tarr (2003) reminded us that, Curriculum is often covered at a superficial level rather than lived in and around questions that truly engage children. Curricula need to take up children's questions rather than ignoring or glossing over their issues. This need to shelter and protect children is closely related to images of children that have been constructed by the field of developmental psychology" (p. 7).

Through hosting, introducing, displaying, exhibiting and offering repurposed materials, Remida reuse education is distinctively early childhood education for sustainability (Reggio Children, 2005).

Interestingly, Grieshaber and Cannella (2001) advocated that another way in which educator identities can be understood as more complex than the dualism generated by developmentally appropriate versus inappropriate discourse is by examining the 
literature on advocacy in early childhood education. Grieshaber and Cannella (2001) point to literature that has supported the notion of educators being activists for children, families, and the field, while at the same time sending a mixed message regarding the image of the educator. Grieshaber and Cannella (2001) described the contradiction as such, "The advocacy literature constructs educators as confrontational, demanding, willing to engage in conflict, and open to both critique and negotiation. However, developmentally appropriate practice positions teachers as those who quietly respond as caring, nurturing, and facilitative of children's development” (p. 16). This incongruent concept in defining the image or identity of the early childhood educator invites dialogue about the ways that education theory has limited educators' identities and the epistemic spaces created for the social construction of counter-identities.

Moreover, young children under five years of age are exploring moral and democratic issues and clearly articulating how they would like their lives and communities to be (Davis, 2008; Johansson, 2009; Mackey, 2012). Grieshaber and Cannella (2001) point out that it is therefore imperative that early childhood educators who identify as social constructivists dialogue with one another about how to socially construct identities with preprimary age children in rapidly changing climate and ecosystems.

The newly emerging education for sustainability (EFS) focuses more intently on the pedagogies of humans as agents of change; whereas environmental education has tended to focus more on green issues such as nature conservation and connecting humans with nature (Elliot \& Davis, 2009). Fostering ecological and democratic agency is a 
common thread amongst educators who seek to change the consumption culture at a young age (Parnell, Downs, \& Cullen, 2017).

To emphasize the literature gap on ecological studies in early childhood education, Davis (2009) undertook a preliminary survey of literature between the years of 1996-2007, in which he looked at a number of international research journals in environmental education and early childhood education. Davis searched for studies at the intersection of environmental education and ECE. Very few were found; in fact, less than $5 \%$ of published articles over a 12 -year period looked at environmental education within the context of early childhood education settings. In general, early childhood education researchers have not engaged with ecological issues, and environmental education researchers have not focused their attention on very young children and their educational settings. Of those studies that were identified, Davies (2009) shares, Most explored young children's relationships with nature (education in the environment), a smaller number discussed young children's understandings of environmental topics and issues (education about the environment), while there was an almost total absence of studies that examined young children's learning and capabilities in responding to sustainability issues such as water or energy conservation (education for the environment) . (p.5)

In response to these findings, Davis (2009) identified necessary supports to establish a research base to guide and nurture early childhood education for sustainability as a legitimate new field of research. As early childhood educators begin to engage, it is imperative that their efforts are underpinned and informed by research and that they are 
supported both conceptually and practically. Davis defined conceptual support as "full recognition that the early childhood education sector has something to contribute, both to the development of human capacities that underpin learning for sustainability, and also to education for sustainability more broadly" (p. 18). Her definition of practical support means that "funding and support for research need to be explicitly directed into the field and towards the development of research capacity" (p. 18).

The initiation of ecological sustainability research projects targeted specifically at early childhood education has employed qualitative research designs that emphasize collaborative relationships between educators and researchers based in recognition that knowledge is co-constructed in the process of conducting research (Ritchie et al., 2013). In keeping with the call for collaboration, several recent studies - between the years of 2009 and 2015-have involved meta-analysis of literature participatory case studies, ethnographic and narrative inquiries, indigenous methodologies, pedagogical documentation, participant observation, and focus group interviews. Systems theory, ethics of care, place-based pedagogies and critical theory have contributed powerful frames for interpretations of ecological practices in early childhood education.

Recent research has focused on the bicultural nature, politics and pedagogies that underpin early childhood education in Australia. The Australian national professional association for early childhood educators has recently updated its Code of Ethics to include "the obligation for early childhood educators to "work with children to help them understand that they are global citizens with shared responsibilities to the environment and humanity' (Code 1.4)” (Davis, 2009, p. 230). 
New Zealand is taking up the charge as well. For example, in response to a similar call to action, a participatory case study on environmental curriculum in a city kindergarten in New Zealand resulted in the identification of themes related to the importance of respecting children's rights within the context of early childhood education and the ways that this action enables children to be supported in acting for the environment.

Mackey (2012) outlined the following emergent themes from the New Zealand study: the right to know about how to care for the earth in a "way that is appropriate for their culture, their place of play and learning, and their capabilities" (p. 478); the right to have your contribution valued and heard; the right to find a solution to environmental issues in their school and communities; and the right to act. In acknowledging the contributions of young children, researchers, educators, parents and policymakers, Mackey (2012) concluded that “children's voices are valued and their creative solutions and enthusiasm for action become a force for the further development of early childhood programmes that integrate sustainability and early childhood policies at a local and national level" (p. 483).

Certainly, Parnell, Downs, and Cullen, (2017) point out that many paths can be taken to "foster intelligent moderation in early childhood education" (p. 1) that are locally relevant and culturally appropriate, educating global ecological citizens who are well informed and uphold democratic values. Remida identifies as a cultural education center because its mission is to encourage children and adults to internalize intelligent moderation as a cultural practice rather than a behavioral approach. 
To better understand the pedagogical foundations that educators are either moving from or moving away from, it is helpful to understand ideas that inform mainstream early childhood studies. The next subsection describes developmental theories that have influenced teacher training and pedagogy followed by a description of new avenues of thought that are recently emerging that fit with the goals of fostering intelligent moderation in early childhood education.

\section{Mainstream Early Childhood Identity Studies}

Mainstream identity studies, such as those found in Heroman, Trister Dodge, Berke, Bickart, Clker, Jones, Copley, and Dighe's (2010) book, Creative Curriculum, are typically informed by dominant theoretical milestones of childhood development. International sources such as Palaiologou (2011) describe patterns of development that pre-primary children are supposed to follow according to developmental psychologists. The typical social-emotional characteristics of two-to-five year old's derived from developmental research include: being impatient; finding it difficult to take turns, prone to temper tantrums and difficult to reason with, often defiant, capable of watching and imitating play but seldom interacting directly with other children, bossy with caregivers and parents, unpredictably moody, often appearing selfish, insistent on trying to do things independently, often incapable to view the world from another's point of view, unable to handle things not going their way, and incapable of understanding ethical behavior or moral standards (Hobart, Frankel, \& Walker, 2009).

The nature of identity studies across the human lifespan has changed over time with micro-sociological perspectives and a concentration on individual identity_or a 
"me" focused perspective_-dominating through the 1970s. Up to that point, researchers primarily explored ways in which interpersonal interactions mold an individual's sense of self. Currently, mainstream approaches to identity studies in early childhood education continue to be strongly influenced by this micro sociological perspective, which, presents a significant challenge from a social constructivist point of view because the primary focus remains on individual identity rather than on ways in which interpersonal reactions mold both individual and social identities.

Grieshaber and Cannella (2001) hold that the identity of the public body of knowledge or discipline of early childhood education is hierarchically tied to developmental psychology and its accompanying constructions of children and early education. Furthermore, Tarr (2003) observed that

In North America we have also remained focused on the individual child in relationship to developmental stages or to learning. As well, contemporary early childhood educational practices related to self-esteem perpetuate a dominant cultural notion of "rugged individualism" that is socially and environmentally unsustainable (Cajete, 1994; Wilson, 2012).

For instance, developing and strengthening children's self-esteem has become a major goal for schools. All About Me curriculum packets (activities, lesson plans, and printable templates) are conventional resources for preprimary school educators who want to engage in identity studies in the classroom. Children work from copied pages prepared by the teacher, each producing an All About Me booklet filled with basic 
information about the child's home and family life. Katz (1993) noted that in the customary All About Me activity,

Each page directed the child's attention toward her or his own basest inner gratifications. Each topic put the child in the role of consumer- of food, entertainment, gifts, or recreation. Not once was the child asked to play the role of producer, investigator, initiator, explorer, experimenter, or problem solver ( $\mathrm{p}$. 2).

It seems to be that only with the influence of the social-historical psychology of Vygtosky (1978) have educators looked to the impact of social context on children's learning" (p. 8). Knowledge of child development theory and the latest research in the field of early childhood education guide high-quality social interactions to benefit the individual academic success of each student. Numerous books, kits, packets, and newsletters produced for teachers urge them to help children gain positive self-concepts. However, many of these materials are didactic curricular templates for identity studies with 3, 4 and 5-year-old children and are not congruent with an appropriate practice grounded in a social constructivist theory of learning (Katz, 1994).

For example, a significant trend in early childhood education is the apparent confusion between narcissism and identity studies. For instance, The Creative Curriculum is a mainstream standardized curriculum authored by Heroman, et al. (2010) used in early childhood education classrooms). The authors stated, "The Creative Curriculum for infants and toddlers focuses on building relationships by fostering a sense of security and self-identity during the first three years of a child's life" (p. 3). This 
message is problematic because it speaks to fostering social relationships in the context of promoting individual academic success in a competitive spirit. As important as it is for children to have high self-esteem, many of the practices advocated in the service of self-esteem, self-regard, self-concept, self-worth, and self-confidence mistakenly develop narcissism in the form of excessive preoccupation with oneself instead of a deep and meaningful sense of individual and social identity (Katz, 1993).

\section{Social Sustainability}

A dualistic understanding of developmentally appropriate and inappropriate practice dominates early childhood education and is particularly prominent in varying opinions regarding early childhood education for sustainability. According to Grieshaber and Cannella, 2001, “This same notion of human structures has been/is responsible for the construction of human 'identity' as a concept, as well as for the creation of individualistic and static interpretations of identity" (p. 8). By emphasizing individual identity to the exclusion of ecological identity, these approaches reinforce the unsustainable historical principle of rugged individualism in social affairs; a belief grounded not only in personal liberty and self-reliance but also in more capitalist values of free competition and excessive consumerism.

Mainstream approaches to identity studies as typified in All About Me curricula are grounded in modernist and structuralist theories of early childhood such as in Freudian psychoanalysis (1930), Sausserian linguistics (Joseph, 2012), early Piagetian psychology (1958). Modernist knowledge is viewed as universal and is associated with industrialization and individualism. Pruett (1999), a clinical professor at Yale 
University's Child Study Center, epitomized this image of the child when he declared that a budding sense of self becomes the major developmental issue at 18 months. Like many developmental psychologists in the 1990s, Pruett held that "The self — the ME—of the young child emerges in this 18-month era with such force that it feels more like a geologic event than a stage in development.” (p. 52). In his chapter on ages and stages, Pruett wrote, "The hallmark of this period is the birth of the self — awareness of 'me' versus 'not me' in body, heart and mind" (p. 202).

In response this this developmental perspective, curricula materials such as the All About Me themed activities persist with an excessive preoccupation with self and selfgratification and include: Me art; me games; me language; me science; me snacks; me music; and me books which include Just Me by Ets (1978); All by Myself by Mayer (2001); and I Like Me! By Carlson (1990). All About Me units such as "I am Special" kits and many similar teaching aids in early childhood education all over the U.S are widely used and inspired by individual and ego-centric theories of developmental psychology.

Another example of mainstream discourses of early childhood identity is Zero to Three (2004). The National Center for Infants Toddlers and Families website suggests families, caregivers and educators of children twelve to twenty-four-months facilitate a toddler's self-awareness as the understanding that she is separate from you and that she is her own person (find more information at https://www.zerotothree.org/). Standard Pre-K curriculum resources for identity studies with children epitomized by popular All About Me templates re/produce values of individualism and are didactic in terms of devaluing 
social identity in favor of individualism. Examples include poems like “I'm Glad I'm Me" included in All About Me resources:

I'm Glad I'm Me

No one looks the way I do.

I have noticed that is true!

No one walks the way I walk.

No one talks the way I talk.

No one plays the way I play.

No one says the things

I say.

I am special!

I am ME!

There's no one else I'd rather be!

And songs like "Everybody says":

Everybody says

I look just like my mother.

Everybody says

I'm the image

of Aunt Bee.

Everybody says

My nose is like my father's.

But I want to look like ME

(preschool.uen.org/curriculum/Septembers/AllAboutMeUnit.pdf)

According to Katz (1993), early childhood educators who use All About Me

booklets express a common belief. They believe the intentions behind the commonplace All About Me exercises are to enhance children's sense of self and to motivate them by beginning where they are developmentally speaking. For example, asking children to describe behavioral and dispositional qualities about themselves such as the color of their eyes, their age, their address, which hand they write with, their favorite color, and their favorite things. 
Alternatively, Katz (1993) poses research questions on contemporary early childhood educational practices related to self-esteem and narcissism such as, "Why should children's attention be turned so insistently inward toward themselves? Can such superficial flattery boost self-esteem? Can young children's minds be intellectually engaged by such exercises?” (p. 13)

Katz (1993) has suggested that while everyone may be special, as educators we surely want to foster in children the view that, while we are unique in some respects, we also have a great deal in common. She reasoned that the same intentions for starting where children are can be satisfied in other ways such as, "By providing topics that would encourage curiosity about others and themselves, reduce emphasis on consumer activities, and at the same time strengthen the intellectual ethos of the classroom. Indeed, starting where children are can just as easily be satisfied by pooling the class data in a project entitled 'All About Us"' (p. 13).

Katz's work was instrumental in opening mainstream North American educators to considering a socially constructed view and alternative approach to identity constructions. Emerging identity theories in sociological, postmodern, and social constructionist literature refocus attention from the individual to the collective (Cerulo, 1997; Project Zero \& Reggio Children, 2001).

For example, Borderlands cultural theory (Anzaldua, 1987; Anzaldua and Keating (Eds.2002)) suggests a range of identities that go beyond dualisms that can be applied to different ways of thinking about early childhood education in a scattered, diverse and mobile society. That said, creating space for diverse epistemologies of early childhood 
education does not necessarily mean rejecting developmental psychology but rather involves an expansion of perspectives.

For instance, Davis and Elliot (2009) noted that contemporary early childhood researchers attribute social constructionist, post-modernist and poststructuralist perspectives as informing the liberation of early childhood from constricting conceptualizations of what is "natural, normal, and necessary" (Woodhead, 2006, p. 21). Egan (1988) argued that even very young children are concerned with the abstract themes such as power and control and issues surrounding what it means to be human, ideas that are usually thought to be topics to be excluded from early childhood education.

Tarr (2003) encouraged early childhood educators to embrace one of the focal points of the Reggio Emilia philosophy, a socially constructivist image of the child. Tarr (2003) encouraged a critical awareness, "The hegemony of developmental psychology on our understanding of children has restricted our vision of them in relation to developmental stages. These are seen as universal and natural stages irrespective of context and culture" (p. 7, 2003). Rinaldi (2006) stated, "What we believe about children thus becomes a determining factor in defining their social and ethical identity, their rights and the educational context offered to them" (p. 65). She uses the words image and identity interchangeably regarding the child and underscores how our definition of the image or identity of the child is above all a cultural, social and political convention. Rinaldi told us that in contrast to traditional ways of thinking about the early childhood developmental stages, social constructivist educators recognize that the young child is 
more than just a sponge but rather is "a producer of culture, values and rights, competent in living and learning” (p. 65).

Encouraging preschool children to distinguish and differentiate themselves from their own families and others as a means of constructing individual identity is perplexing from a social identity and social constructivist perspective. As Vygotsky (1978) stated, “Through others, we become ourselves.” (p. 38). Family systems theory (Bowen, 1978) suggests that individuals cannot be understood in isolation from one another, but rather as a part of their family, as the family is an emotional unit. Indeed, families are the first culture group in which children perceive membership (Carlo, Crockett, \& Carranza 2010).

In fact, contemporary social constructivist early childhood education tends to highlight how family is the most basic element of self-awareness for young children. Family shapes and informs the sense of self a child forms and who that child feels like she is in the world. The feeling of belonging, in the widest sense, contributes to inner well-being, security, and identity (Weiss \& Lopez, 2015). Therefore, it is important for children to see their families reflected in the classroom, while at the same time seeing the diversity of families that also exists in our preprimary schools (NAEYC, 2009; GonzalezMena, 2008).

Again, these notions harken back to Tarr's (2003) observation that developmental psychology has hindered our relationship to human growth within specific cultural contexts; she advocates that these ideas must intertwine as we find so often in the work of social constructivism. For example, the Reggio Emilia image is of a child "who is able to 
assemble and disassemble possible realities, to construct metaphors and creative paradoxes, to construct his own symbols and codes, while learning to decode the established symbols and codes" (Rinaldi, 2006, p. 65). The notion of the child as a cocreator and producer of culture is central to Reggio-inspired early childhood education and to this research project.

Tarr (2003) challenged, "If children are to be creators of culture, and their interests, theories, questions, concerns, are to become part of school life, how can we set the stage for that to happen?" (p. 9). In response to this challenge, Remida-inspired education creates space for young children's agency as global ecological citizens.

Creative Curriculum research also shows that early pro-social behaviors, such as cooperating, consoling, helping, and sharing, predict later academic achievement" (Heroman, et al., 2010, p. 5). This traditional developmental perspective is echoed by The Goddard School for early childhood development. To set the stage for each child's individual achievements, trained teachers observe and assess a child's developmental accomplishments. These guidelines are based on research on how children learn best, state and national standards and results from national learning assessments. The Goddard School teachers plan fun, engaging activities that build upon what children can do, encouraging exploration and fostering self-esteem. The curriculum intentionally guides and extends a child's play to ensure children achieve key developmental milestones across these seven learning domains including personal and social development. 


\section{Social Constructivism as a Theoretical Basis of Early Childhood Education}

Piaget (1952) and Vygotsky (1962) are two eminent figures in the development of constructivist theories. Piaget's (1970) later work on social interaction theory noted that cooperation is a factor in the creation of personality as a stable ego. Piaget (1970) posited that personality is the result of continuous interaction with others through comparison, opposition, and mutual adjustment. For the development of reason and morality, Piaget theorized that cooperative relationships are necessary. For Piaget, therefore, cooperation is an essential characteristic of developmentally oriented education not simply because it is a culturally valued virtue, but because of its psychodynamic developmental significance.

In Piaget's work of the 1940s and 1950s, even when he was focused on the construction of cognitive operations, he went further to postulate that progress in social development and the development of logic "go completely hand in hand and constitute two indissociable aspects of a single reality that is at once social and individual" (Piaget, 1945, p. 145). Thus, we see in his general statements a deep regard for social factors as equal to cognitive factors in a child's development.

More recently, Mooney (2013) noted that rather than asking what children know and or when they know it, Piaget asked how children arrive at what they know. Mooney stated, "The world children inhabit is shaped by their families, communities, socioeconomic status, education, and culture. Their understanding of this world comes, in part, from the values and beliefs of the adults and other children in their lives" (p.100). Mooney (2013) reminded us that Piaget's developmental theory of learning and 
constructivism is based on discovery. According to Piaget's constructivist theory, to provide an ideal learning environment, children should be allowed to construct knowledge that is meaningful for them.

Vygotskian theory represents a shift from the cognitive to the social-cultural. The social lens that drove Vygotsky's theory of the nature of human cognition was deeply influenced by his strong admiration for Marxist philosophy. The sociocultural theory as developed by Vygotsky places emphasis on the social nature of human mental activity. One of the main contributions of Marxist influence on Vygotsky's view on language and mind was that human thought was fundamentally a dialogical rather than mechanistic phenomenon. For Piaget, language is secondary to action; thought precedes language. Alternatively, Vygotsky (1978) posited that the development of language and thought go together and that the origin of reasoning is more to do with our ability to communicate with others than with our interaction with the material world. Vygotsky's (1979) statement, "The social dimension of consciousness is primary in time and fact. The individual dimension of consciousness is derivative and secondary" (p. 30) captures his sociocultural theory of human cognition.

Making even stronger connections between social constructivism, identity, and the Reggio Emilia approach, Malaguzzi (1993) reminded us that "relationship is the primary connecting dimension of our system [in Reggio Emilia]. Among the goals of our approach is to reinforce each child's sense of identity through a recognition that comes from peers and adults, so much so that each one would feel enough sense of belonging and self-confidence to participate in the activities of the school" (p. 45). The construction 
of the first school-built brick by salvaged brick in 1947 was the symbolic building of a new society where no one is disconnected.

Hawkins (1997) compared the history of Reggio Emilia, Italy and its trajectory of communitarian existence through permanent, deeply rooted settlements since ancient times in Europe to the largely uprooted cultural experience in North America, "We in the United States do not know such solidarity, such sustaining communality, reshaping itself in ways Malaguzzi describes, demanding better education for children” (Edwards, Gandini \& Foreman, 2012, p. 77). The roots of Reggio Emilia municipal schools are grounded in an inclusive image of children as citizens who have the democratic responsibility and right to be part of the cultures, school, and town in which they live.

A social constructivist approach focuses on the ecological contexts in which children negotiate their identities. In social constructivism, learning is considered a social process and meaning is an agreement shaped by social patterns and the assumptions encapsulated in language. Thus, in the spirit of Reggio-inspired education outside of Reggio Emilia, Italy, experimentation and re-interpretation of the principles offer useful reference points in a way that allow for adaptations that are locally relevant and culturally appropriate (Wien, Gyuvesky, \& Berdoussis, 2011). The Reggio approach explicitly recognizes that it is important to experiment and re-interpret social identity through Reggio-inspired education in a way that is locally relevant and culturally appropriate to a scattered, diverse and highly mobile North American society that has deep roots in individualism also characterized by budding understandings of socially constructed identity in educational settings. 
The concepts of semiotics and the use of metaphor are the integral to the Reggio approach (Vecchi, 2010) as they aid in the construction of symbolic thinking, social interaction and cultural awareness. Vecchi (2010) suggested, "Constructing metaphors is a mental operation giving unusual, unexpected and very often totally original results. I believe metaphor corresponds to an investigative attitude towards reality, to participation that allows our thoughts to open out and break down the rigid boundaries that are usually constructed" (p. 34). In this regard, metaphorical interpretations lend themselves to a Borderlands paradigm.

By facilitating opportunities for children to develop and draw upon metaphors Chaillé (2007) conceptualized the early childhood educator as a "facilitator of learning" (p. 47) as opposed to a transmitter of knowledge. She described the socially constructivist educator as such, "The teacher is the core, the orchestrator, the creator of an environment in which learning thrives" (p. 47). She clarified this distinction stating: In some ways, this lifts a tremendous burden, since the teacher is no longer the source of information, whose presentation make or breaks the outcome. But the teacher's responsibilities are in no way reduced in a constructivist classroom; rather, they are shifted. No longer the source of information, the teacher must instead play many different roles. The responsibilities are more numerous and more complex. (p. 47)

Participants in this study related their constantly shifting role in the classroom in a similar manner, describing their work as socially constructing identities. Next, I will present the literature that describes the concept of socially constructing identities. 


\section{Socially Constructing Identities in Early Childhood Education}

The study of social identity is a major sociological, psychological, anthropological and educational construct (Bennet, 2004, 2011; Brooker \& Woodhead (Eds.), 2008). Early childhood educators who identify as social constructivists engage an understanding of the effects of cultural, social, and economic diversity and variations of individual growth to inform identity studies with young children. Malaguzzi (1996) reflected on this notion of "The importance of seeing yourself again" (p. 46). He stated, ...a child's most sought-after goal is to recognize himself in others, and to find in others (objects and the natural world as well) parts of himself...contradictory images, resources and values filter down to the child, and discourages this sense of one's own self; a quality that the child himself must set in motion as soon as he can, with adult help and cooperation. These notions appear also in the face of violent existential and cultural pressures that society places on people, especially on the very young, with the risk of making them anonymous, alienating them. (p. 47).

Malaguzzi's notion here is like Anzaldua's (2009) in that "Identities are constantly under construction" (Keating, 2009, p. 226). Malaguzzi acknowledged the reality of living in a socially constructed world and encourages children and adults to socially construct and reconstruct a sense of one's own authentic selves.

Further, Cerulo (1997) noted how sociological studies of identity in key works published from 1980-1997 reveal that "Sociologists focused primarily on the formation of the 'me' exploring the ways in which interpersonal interactions mold an individual's 
sense of self" (p. 387). In contrast to that prevailing focus, more recent literature refocuses attention from individual to social identity or the collective. Collective identity is the belief that through participating in social activities, individuals can gain a sense of belonging and an identity that transcends the individual.

Interestingly, although the emphasis on collective identity is new in early childhood education, this concept has roots in traditional sociological constructs. On one hand, sociology has historically attributed the nature of collective identity to biological characteristics. Anti-essentialist perspectives on the other hand, maintain that the social constructions of identity are a more viable basis of the self.

Seminal research in early childhood education reflects the shift toward constructing individual identity in the context of social and ecological identity. For example, Project Zero (2001) was carried out collaboratively by the Harvard Graduate School of Education and Reggio Children. This project involved the voices of educators, young children, parents and pedagogical coordinators from the Municipal Infant-Toddler Centers and Preprimary schools of Reggio Emilia. The hypothesis set forth by Project Zero and Reggio Children is that making learning visible through documentation can foster both individual and group learning, creating a relationship between them.

One of the many findings that emerged from Project Zero was an observation that sociocultural and ideological shifts in identity theory were reflected in these early childhood educators' approaches to identity studies with young children that highlight the complexity and social embeddedness of identity and early childhood educational practices. As Haslam (2004) a co-researcher on this project noted, "The capacity to think 
in terms of 'we' and 'us', and not just 'I' and 'me' is central to the capacity for meaningful group behavior" (p. 17). The hypothesis set forth by Project Zero and Reggio Children is that making learning visible through documentation can foster both individual and group learning, creating a relationship between them.

In epistemological discussions of identity formation and construction, one controversial issue has been whether individual identity informs social identity or vice versa. Some hold a dichotomous view of individual and social identity and argue that one develops or constructs the other. Like Gardner (1983) the Project Zero group maintained a perspective congruent with a Borderlands paradigm whereby, "The construction of self, of the individual, becomes increasingly defined as a point of intersection and of multiple identities. More and more, the individual will express an intercultural, intersubjective identity" (Project Zero and Reggio Children, 2001, P.45). Malaguzzi (1920-1994) similarly articulates a Borderlands-type approach to the relationship between individual and social identities as he stated in the book Reggio Children (Eds,1996), A child is born a first time, and then, through the long and difficult process of constructing his identity, it is as if he is born again. In this process, he give himself a face, a body, gestures, movement, speech, thought, feelings, imagination, fantasy; in short, the awareness of being and the means of expressing his "me-ness" which are absolutely essential for becoming autonomous and distinguishing ourselves from other people and things - people and things we live and interact with and from which, little by little, we draw most of the raw material with which we create our own identity. (p. 47) 
Reggio-inspired educators believe that identity is a social construction, shaping and being shaped by the school (Chaillé, 2007; Dalhberg, Moss, \& Pence, 1999; Fraser, 2000; Fu, Stremmel, Hill, 2002; Helm \& Parnell, 2010). Similarly, Vygotsky (1978) commented, "Every function in the child's cultural development appears twice: first on the social level, and later, on the individual level; first, between people (inter-psychological) and then inside the child (intra-psychological)" (p. 57).

Studies that concur with the claim that early childhood programs have lasting effects on children later in life and that the most beneficial early childhood programs are based on a constructivist theory of learning and constructivist practices (Dunn \& Kontos, 1997; Marcon, 1994; Peck et al., 1988; Schweinhart, Weikart \& Larner, 1986; Stipek, Feiler, Daniels, \& Milburn, 1995). This explanation from Devries and Kohlberg (1987) encapsulates this theory of learning, "These ideas must therefore come from the childfrom his or her own effort to make sense out of experience" (p. 18). This is what Piaget (1945) meant when he said that the child constructs knowledge. Devries and Kohlberg (1987) added, "When we take the trouble to find out how children actually think, we find that they learn— that is, construct—many things we do not teach" (p. 18).

This study aligns with the view that appropriate practice for identity studies in early childhood education is grounded in a social constructivist theory of learning (Grieshaber \& Cannella, 2001; Edwards, Gandini \& Forman, 2012). In keeping with this theoretical perspective, as a Reggio educator and researcher, I embrace this interdependent approach to identity studies that socially constructs individual selfhood me in early childhood within the context of ecological selfhood we. 


\section{Implications of the Literature Review}

This research study documents the history of developmental perspectives related to early childhood and new and evolving epistemological spaces created by Reggioinspired educators who socially construct identities with children using reclaimed materials. The chapter explored innovative theories of social and ecological learning examined in the early childhood education for sustainability, Remida Center and Remidainspired materials literature in order to challenge traditional developmental theories about the formation of children's identities.

In this examination of the literature that informed this study, I described mainstream perspectives. I also challenge a constricting image of the child through exploring documentation of preprimary age children's ability to work with complex moral, ecological and democratic issues across a range of contexts within their everyday social interactions. Overall, this literature review illuminates Reggio-inspired educators' perspectives of pre-primary age children's competence in socially constructing complex knowledges through representing their theories on identity and ecology with reuse materials.

Reggio-inspired researchers speak to how critical engagement with complex issues like self-concept as social beings generate space for new understandings of how early childhood education can contribute to theory and practice of identity studies. Reggio-inspired research focuses in a new way on social identity, through the creative use of Remida-inspired materials. The idea is both to facilitate representational thinking, agency (how we move and are in the world) and demonstrate our core values and beliefs 
in two and three-dimensional representations. My aim is also to raise awareness about the origins of materials, essentially recycling otherwise unused materials and putting them to use in creative ways to document expressions of children's self-concept individually and collectively.

This literature review introduced the heterogeneity of social constructivist perspectives and approaches to social identity. Importantly, this chapter also described the idea that Reggio inspired schools are heterogeneous as well. The Reggio inspired Remida Centers involved in this study adapt instructional strategies to fit the sociocultural and environmental context they are working in rather than attempting to adhere to the Reggio Italy's approach with a high degree of fidelity.

Now that I have spent time describing the literature that informed my study, I will turn toward Chapter 3. This chapter will describe the methodological approach, the methods used in this piece of research, data collection, and the interpretation strategies employed in this dissertation. 


\section{Chapter 3: Methods and Approaches}

The purpose of this dissertation is to conduct narrative inquiry research into Reggio-inspired early childhood educator's experiences of socially constructing identities with children using reuse materials. Throughout this study, I use the terms social and ecological identities interchangeably, with a focus on ecological identity as the social construction of a profound sense of oneself in relationship with natural and social ecosystems. Remida cultural education socially constructs identities in the Municipal Infant and Toddler Centers and Preprimary Schools of Reggio Emilia, Italy by giving value to waste materials through its teaching methods, which include hands-on proposals for expressing young children's work and thinking. There is a lack of literature connecting children's social identity constructions and early childhood education for sustainability.

Overall, developmental psychology has been primarily concerned with the initial emergence of the self-concept rather than the co-construction of identities in ECE. Early childhood developmental research directed at the personal self is problematic from a social identity approach whose orientation is on the development of the social self. In this study, I explored the new and evolving epistemological spaces created by Remidainspired educators, outside of the context of Reggio Emilia, Italy.

Chapter 3 provides a description and justification of my research design and methodology and offers a review of related methodological literature. Here, I describe my role as a co-researcher who worked in collaboration with the participants in the study 
to inform the research question: What are early childhood educators' experiences with reuse materials in Reggio Emilia inspired identity studies?

In the research design subsection, I identify the types of participants involved and the participant recruitment processes. I also outline the connection between the chosen critical perspectives, the research question, and narrative inquiry approach used to involve dyadic interviews and a focus group. Next, the research methods are discussed including the intentional setting of the study, procedures used for data collection, and interpretations. The chapter concludes with a statement of positionality, followed by a chapter summary.

\section{Methods and Approaches}

This narrative inquiry study was exploratory in nature. In fact, I only found two pieces of published research involving focus group interviews with Remida-inspired educations related to reusing materials with young children (Eckhoff et al., 2011; Parnell et al., 2017). These studies each encompassed assemblage art and why educators deem its techniques appropriate to be incorporated in early childhood curricula following the Remida approach. The authors drew from focus group interviews to describe the origin and theories of the instructional approach and how creative reuse education can be taught using key ideas. A key point they made demonstrated that Reggio Emilia-inspired approaches to early childhood education are relationship-based. Therefore, interactive experiences designed to elicit Reggio-inspired educators' observations and reflections in a group or dyads (pairs) is complementary to the socially constructivist pedagogy they practice as co-teachers. 
In my qualitative study, I conducted a narrative inquiry based on dyadic interview data and a focus group discussion with six educators who explicitly identify as socially constructivist and who are involved in identity studies and creative recycling with children 2 to 5 years of age in two Reggio-inspired settings. Here, I present methodological literature upon which this study was based.

\section{Review of the methodological literature.}

A review of the methodological literature revealed that narrative inquiry can effectively illuminate the lived experience of early childhood educators. Certainly, similar methodology has been used in studies having similar purposes to this type of research (Cavendish, 2011; Trahar, 2013). I begin this section by introducing narrative inquiry as a method of research and justify the selection of this method for my study. Then I review Sfard and Prusak's (2005) narrative theory of identity as it relates to theoretical conceptualizations of identities and as an analytical tool useful for "fathoming the mechanism" (p. 19) of identity formation and learning in my study.

Narrative Inquiry (Clandinin \& Huber, 2016; Connelly \& Clandinin, 1990;

Cresswell, 2015; Hunter, 2010; Maple \& Edwards, 2010; Maynes, Pierce \& Laslett, 2008; Wells, 2011) is described as:

...the study of the ways humans experiences the world. This general concept is refined into the view that education and educational research is the construction and reconstruction of personal and social stories; learners, teachers, and researchers are storytellers and characters in their own and other's stories (Connelly \& Clandinin, 1990, p.2). 
Originated in literature, narrative inquiry focuses on human experience. This approach has a long intellectual history in other disciplines including sociology, psychology, literature, sociolinguistics, anthropology, and education. Since the 1950s, there has been a large increase of scholarly work based on "personal narrative evidence - that is on retrospective first-person accounts of individual lives" (Maynes, et al, 2008, p. 1). Narrative can be both a method and the phenomena of study (Connelly \& Clandinin, 1990; Pinnegar, 2006). A well- established practice is to call the phenomenon story and the inquiry narrative.

In keeping with a prominent Chicana approach to social science research, I chose narrative inquiry because it provides access to marginalized voices and allows for counter-narratives. Anzaldua (2009) coined the term autobiographical theory or autohistoria-teoria, which signifies a transformation of traditional Western autobiographical forms informed by reflective self-awareness in the service of social justice work. Autohistoria focuses on the personal life story but, as the auto-historian tells her own story, she simultaneously tells the life stories of others (Keating, 2009; Sandoval, 2007). The concept demonstrates that epistemology of self-knowledge practices, like all knowledge practices, is social and relational (Pitts, 2016).

This narrative inquiry choice, and my research question (What are early childhood educators' experiences with reuse materials in Reggio Emilia inspired identity studies?) were inspired by the work of Maynes, Pierce, and Laslett (2008) who said that interpretations of personal narratives can reorient theories about the relationship between the individual and society by calling attention to the dynamics through which individuals 
construct themselves as social actors. In this way, "They have the potential constructively to intervene in the theoretical impasse resulting from the collision between skepticism of hegemonic individualism, on the one hand, and the persistent, even increasingly urgent interest in understanding selfhood and human agency, on the other" (p. 2). The notion of personal narratives as documents of social action and selfconstruction is highly congruent with my research focuses on identity and social constructivism.

Identity in research methodology. The work of Sfard and Prusak (2005) informed this research who investigated the sociocultural forces that make people who they are as evidenced through their actions. In fact, Sfard and Prusak provided the following operational definition used here for the notion of identity, "A set of reifying, significant, endorsable stories about a person" (p. 1).

Sfard and Prusak (2005) created a postmodern framework of identity construction within the field of social constructivism, revealing a narrative methodology amenable to empirical research. This postmodern work questions essentialist notions of identity as singular and unitary. In contrast, a postmodern understanding of identities is that they are multiple, complex, and fluid (Grieshaber \& Cannella, 2001). Young children's identities are multiple and complex (Raburu, 2015). Brooker and Woodhead (2008) state that changing cultural and social contexts, and new relationships, allow individuals to develop new or modified identities. "There is therefore a tendency to understand identity as a multiple construct or to describe individuals as acquiring multiple 'identities"' (p. 10). Multiple identities exist for any person and the nature of identity is dynamic and 
predominantly a lived experience (Sfard and Prusak, 2005). Rather than suggest identities find their expression in stories, these narrative inquirers claim identities are stories. An individual's various identities are distinguished by markers such as an identifying story told by the person herself (first-person identity); an identifying story shared with an identified person (second-person identity); or a story told about an individual by a third party to a third party (third-person identity).

There has been a renaissance in multiple disciplines around the concept of identity, including cultural studies and educational research. Conceptualizing identities as a source of data is grounded in interpretations of stories about persons. Sfard and Prusak (2005) argued that providing space for people's inner forces to emerge in their narrative theory of identity is a powerful analytical tool for understanding the cultural shaping of learning. As is the case with this study, these scholars explore identity as a human construct, constantly created and recreated in interactions with people. Their thesis is that the concept of identity itself is the missing link in a complex dialectic between learning and its sociocultural context.

At the same time, the authors acknowledged that language tends to resist innovations. This presents shortcomings in qualitative research such as what language can be used. This has been cited as a major challenge in postpositivist research (Castillo, 1994). Since language can be a limitation in eliciting nuanced and complex ideas, the Reggio Emilia experience fosters intellectual formation through a systematic focus on symbolic representation. Likewise, in this study, the participants were encouraged to 
explore their environment and express themselves through the languages of reuse as a tool for eliciting perceptions and expressing theories.

Conceptualizations of self-knowledge as a cultural and epistemological practice (that are valued in Chicana/o studies and in Reggio-inspired learning communities) are further developed in this narrative inquiry, which focuses on how early childhood educators perceive that Remida-inspired creative experiences influence the formation of children's social identities. In his final years, Malaguzzi (1990) stated, “Certainly even today if we put all the keys in our pockets together they cannot produce the right image of children" (Malaguzzi, 2016, p. 368). He spoke to the image or idea of the child as fluid in nature and of young children's identity formation as a dynamic and predominantly lived experience.

Moss (2016) elaborates, "Leaving room for the unpredictable, the surprising, happenchance, without which our species would be terribly depleted, perhaqps no longer exist, is vital for children and for human beings, while pedagogy and schools think they have to put the brakes on their play" (p. 368). This notion of childhood as having an identity also demonstrates that self-knowledge practices are social and relational. Moreover, such self-knowledge practices require contestation and affirmation as well including resistance and creative friction.

From a social constructivist paradigm perspective, Sfard and Prusak (2005) criticize scholarship that renders ambiguous notions of identity as timeless and agentless or even static. They make the invaluable scholarly contribution of defining identity to "make the notion operational, immune to undesirable connotations and in tune with the 
claim about identities as man-made and collectively shaped rather than given" (p. 3). Here, conceptualizing identities as a source of data is grounded in narrative interpretations of stories about persons based on the belief that collecting and analyzing stories can reorient theories about the relationship between the individual and society by calling attention to the social and cultural dynamics through which individuals construct themselves as social actors.

\section{Narrative inquiry for investigating notions of identity formation.}

Another key reason I selected a narrative inquiry methodology for this research was that the narrative perspective is congruent with Sfard and Prusak's (2005) operational definition for the notion of identity as an analytic tool for investigating learning. Sfard and Prusak conceptualized identity as "a set of reifying, significant, endorsable stories about a person" (p. 1). Narrative inquiry served to illuminate my research participants' experiences and prevented generating themes that are generic but not representative of the individual voices of the educators in the participant group.

Narrative methodology is a natural choice for a broad methodological stance because of the distinction that narrative inquiry can offer in that it helps to avoid mining data for themes, thus avoiding depersonalizing and decontextualizing the stories from the participant (Clandinin \& Huber, 2016). The critical pedagogy borne of the epistemological curiosity of Chicana/o studyists can be quite different from the perspective of mainstream academics. New theories of knowledge allow Chicana/o studyists to interrogate the social situation in a novel way (Delgado Bernal, 1998; Pizarro, 1998). Esmeralda de los Santos (2005) observed that "limited research has been 
conducted on Chicanas in general and has focused on quantitative research methodology that fails to capture the nuanced descriptions needed to describe such concepts and processes as border crossings and multiple identities" (p. 107). Embedded in Borderlands and Xicana (Chicana feminist) epistemes are diverse ways of investigating the origin, nature, methods, and limits of human knowledge. At the same time, "This approach to writing not only represents a way to analyze the data, but also demonstrates how narratives can inform the work in a way that honors the whole" complex nature of identity (Maple \& Edwards, 2010, p. 33).

For example, narrative inquiry frequently involves the "use of metaphors to unlock data" (Maple \& Edwards, 2010, p. 40). Reggio-inspired educators often work in the realm of metaphors to describe everyday experiences in the classroom. Socially constructing knowledge in early childhood education lends itself to methodological flexibility useful for analyzing and interpreting multi-metaphorical meta-frameworks and communicating unconventional data and theories. For example, Malaguzzi (1980) made a series of public statements, invitations, and suggestions that illustrate methodological flexibility to address what Freire (2004) termed an "epistemological crisis" leading to new epistemological spaces created from ecological perspectives. Malaguzzi (1989) stated in a speech given at Marzo-Aprile Pedagogico, "It seems to me the first issue is the crisis, or the end-yes, I think the end- of sciences as a teleological reference point, the inflexible source, all-knowing, all-foreseeing." Instead, he suggested, "Today the unpredictable is a category of science." And therefore, 
What is necessary on our part — the time has come — is to analyze the significance and above all to understand the significance that the development of science, the development of technology, of information, of economy, of consumption, all have for reformulating our image of the child and humanity, and of cognitive, ethical and social strategies.

This study engages methodological flexibility useful for analyzing and communicating unconventional data and theories through multi-metaphorical interpretations of narrative data contextualized by meta-frameworks including Borderlands theory and interdisciplinary paradigms. The interconnected, elastic, and flexible nature of narrative inquiry through hands-on reuse provocations, dyadic interviews, and focus group discussion supported the unconventional data and theories that emerged as fluid and hybrid.

\section{Researcher as Co-Researcher}

I embraced Reggio-inspired principles around educator as co-researcher to guide and inform my data collection and interpretation cycles. Rubizzi (2001) re-signifies her role as researcher using the idea of "documenting the documenter" (Project Zero \& Reggio Children, 2001, p. 94). Like her reflections on the identity and role of documentation in an educator's learning process, I wondered about what thought processes orient educators as they document their experience of Remida-inspired identity studies and if an awareness of these processes has value for professional growth. I approached data collection and interpretation of the research as a partnership for learning together with the participants. I went over the research agenda with the participants 
during the recruitment phase, before each interview and focus group discussion, and in individual member checks that followed. In doing so, I was seeking the same co-research experience Gandini \& Edwards (2001) describe as a "cooperative, nonhierarchical, and interactive model of research partnerships, a co-learning agreement” (p. 183). I found Gandini and Edward's democratic approach to data collection appropriate for learning with Reggio-inspired participants.

Comparable to the phenomena documented in research in the preprimary schools of Reggio Emilia, and teacher focus groups that help elicit deeply reflective perceptions, the voices of the participants in my narrative inquiry created what Gambetti described as a "seesaw of dialogues and different points of view" (Project Zero \& Reggio Children, 2001, p. 116) which she describes "is part of a long-term knowledge-building process that leaves open the possibility of revising one's thinking within a situation that is in a constant state of flux, in a search for new identities" (p. 116). As part of this description of my positionality as a co-researcher, I want to clearly delineate the difference between the role of a co-investigator (third-party agents) and my identity as a co-researcher who shares an emic view from within Reggio- and Remida-inspired communities.

I perceive of this study as complex teacher research that records the thought processes orientating the participants as they documented Remida-inspired identity studies through hands-on reuse provocations, classroom documentation, and the social construction of reuse persona dolls in focus groups. As a co-researcher, I simultaneously experienced professional growth through documenting the documenters, so to speak. When teacher research is the core of professional formation, it is both local and creative. 
In addition, it emphasizes the ability to analyze observations and other collected data and to be self-conscious about emotional reactions and pedagogical intentions. Teacher research starts with the stance of reflective practice, which is a stance of inquiry-a willingness to look at one's own practice and realize that he does not know everything and is instead curious and open-minded (Chaillé \& Davis, 2015).

According to Henderson et al. (2012), educators who are teacher researchers become knowledge creators, a radical departure from the way society thinks about educators, particularly in early childhood education where the dominant construction of the educator as expert often prevails. The situated learning perspective of educators as collaborative learners who facilitate learning and reflective practices inspired me to offer participants a chance to reflect on their experiences of Remida-inspired identity studies through the construction of a creative representation together with their dyadic partner in focus groups.

In summary, I embraced Reggio-inspired principles that define the educator as coresearcher to guide and inform my positionality throughout the data collection and interpretation cycles. It is not possible to completely suspend subjective interference; in this study, I interpreted data from an emic perspective as a Remida-inspired center coordinator examining Reggio-inspired colleagues' experiences and perceptions. This study draws heavily on the power of metaphor in a manner that was already comfortable and familiar to me as a Reggio-inspired educator. The research design description that follows demonstrates how I worked with the data in a way that shows my sensitivity to participants' meanings, rather than veiling these sensitivities and denying their existence. 
In effect, I uncloaked the invisible elephant in the room, rather than pretending it did not exist as Rinaldi (2006) might suggest.

So, rather than utterly denying my own subjectivity, the following description acknowledges my sensitivity along the way as a co-researcher engaged in an examination of early childhood educators' experiences with reuse materials in Reggio Emilia-inspired identity studies. That said, using a variety of data collection procedures and triangulating my data improved credibility; the approach used offers a rich and descriptive view of participant narratives while simultaneously reducing the degree of subjective interference within my research (Maxwell, 2005). Rather than seeing triangulation as a method for validation or verification, as a narrative inquirer, I used dyadic interviews, a focus group discussion, and hands-on reuse provocations to ensure that my account was rich, robust, comprehensive, and well-developed.

\section{Research Design}

I begin with a description of the context of this study, followed by a presentation of the procedures used to conduct the study for participant recruitment (see Appendices A and B), informed consent waivers (see Appendix F), and how the data was organized and maintained (see Appendices $\mathrm{C}$ and $\mathrm{G}$ ). My data collection procedures and timeline (see Appendix D) are then depicted (see Appendix E). Afterward, I describe how I practiced creative reuse cultural education using reuse materials to facilitate both personal introspection and the participants sharing of experiences working in a Remida-inspired way around identity studies with the participants. These reuse provocations involved dyadic pairs selected from Remida-inspired materials to represent themselves and each 
other. Offered through a focus group using Remida-inspired materials to make persona dolls, participants personified their ideal of sustainability and reuse educational practices as an embodiment in the doll.

As well, to inform my methodology, data collection instruments are described in more detail—dyadic interviews, sharing documentation, a focus group, and reuse collage artifacts created during discussions. I also share my rationale for the selection of these instruments. What follows is a description of the data interpretation procedures, involving details of the narrative methods used and my metaphorical interpretations.

To begin, this first subsection offers a brief description of the setting of the study followed by a presentation of the context and explanation of the recruitment of participants.

\section{Setting of the study.}

The setting of the study was an early childhood development center (CDC) situated within a public university found in the Pacific Northwest region of the United States (U.S.). I have given it the pseudonym "laboratory CDC" to protect the confidentiality of the school and participants. This laboratory child development center is strongly influenced by the philosophies of the Municipal Preprimary Schools and Infant/Toddler Centers of Reggio Emilia, Italy. Located on an urban campus, the center coordinates with a master's degree program in early childhood education; master's students and work-study students are classroom assistants involved in a clinical immersion experience. 
The center is housed in a historic brick building that has a long history as a preschool that dates to the early 1900s. The building is a combination of historic and modern architecture and sits high up on an embankment surrounded by native landscaping and trails tucked into the urban grid. Traces of young children's ceramic artwork and creative play embellish the exterior. One can often hear the merriment of children playing inside the black wrought iron gates that surround the bountiful play yard when approaching the entrance.

Walking into the foyer of the center which is filled with documentation hanging in the halls provides the impression of entering a vibrant space charged with the theories and agency of young children. Long-term student projects are made visible along dark oak walls through rich imagery and words displayed on panels lining the hallways and staircases. The building has the warmth and grandeur of a historic home coupled with the zest and vitality of emergent research emanating from educators and children interactively learning.

I am impressed by how the values of extended family participation, democracy, and diversity are made highly visible throughout all the wings of the school. As an educator, family member, and/or community member, people commonly share that they experience a strong sense of beauty, belonging, and purpose radiating from all corners of this Reggio-inspired learning community (W. Parnell, personal communication, July 2016). This feeling even extends to the school kitchen, where visual documentation is offered by the cooks on the walls leading into the kitchen that make visible the kitchen staff's identities and relationships to the larger community. 
The laboratory CDC serves 200 children between the ages of 4 months and 5 years old and the center provides students and faculty/staff parents with a high-quality on-campus early childhood education and childcare program. The specific location where the dyadic and focus group discussions were held was in the IRPP identity atelier located on the third floor of the center. This emerging creative reuse cultural project is developing into a center filled with creative, intelligent reuse (Vecchi, 2010) materials that are available to the larger early childhood community.

This IRPP project involves graduate students enrolled in the early childhood master's degree program, the laboratory CDC faculty and staff, and the community in undertaking a cultural project with the aims of reclaiming and repurposing industrial discards and educating community members (especially early childhood teachers) on how to reuse these valuable materials in the classroom, school, and community projects.

The atelier (school reuse studio) setting for the focus group and dyad interviews were an intentional choice based on the concept of the "environment as the third teacher" (Ceppi \& Zini, 1998) in mind. As Orr (1992) suggested, "Knowledge of a place - where you are and where you come from - is intertwined with knowledge of who you are. Landscape, in other words, shapes mindscape" (p. 131). The socially constructed landscape of a Remida-inspired atelier housed in a Reggio-inspired CDC felt familiar and inspired the participants to share how they define and describe the experience of collecting, organizing, and exhibiting repurposed materials to bridge identity studies and reuse materials. There, I collected and interpreted dyad and focus group discussions from 
educators who identify as social constructivist and are involved in identity studies and creative reuse research with children 2 to 5 years of age.

During the dyadic interviews, two participants sat at a table in comfortable cushioned chairs in a room called the Identity Atelier (located in the IRPP center) that was filled with an epistemological display of Remida-inspired reuse materials. There were some snacks and water available at a nearby table. The first two dyadic sessions began with hands-on reuse provocations; a conversation and interactive playing with materials began. To guide the ice-breaker, written prompts such as, "Represent yourselves using materials from the center" and "Share what it was like to represent yourself with reuse materials" were placed on the table during the first session with felt oval materials in different skin tone shades inspired by anti-bias education.

Carts exhibiting a menagerie of colorful and provocative discard as well as natural materials were made available for participants to select from to represent their thinking about identity. For this study, a hands-on reuse collage was defined as an art medium that uses various materials and objects composed over a surface. Collage is often used to help people tell their stories. The purpose of a collage in this research project was to support participants in telling their stories of their experiences in their professional journeys with ecological identity. There were no expectations about the look of the collage - it was a tool for telling their stories (see Appendix B).

After this, each participant was provided with a list of a series of questions intended to guide their conversations. I acted as a discussion guide and began the conversation by asking both participants to consider children, identity, and reuse 
materials. I was present and involved as a facilitator during the first two sessions with the studio teachers and during the first session with the director dyad. Being a participant observer and guide became an awkward challenge because the studio teacher and director pairs continually looked to include me in their conversations. As a result, during the first two classroom teacher dyadic sessions I introduced the question prompts and restated the purpose of the discussion and came and went from the room, but with the participant's permission, I left a recorder and was also listening in on the conversation. I followed this same protocol during all of the hands-on reuse provocations parts of the interviews and during the focus group discussions.

I worked with three pairs of educators and a group of five of the educators served as my focus group. The focus group involving five of the six participants was held in a conference room located across the hall from the Identity Atelier in the IRPP and was set up similarly for the purposes of simultaneously reducing anxiety and promoting creative tension and provocation with materials and with other participants. The playfulness of the decor was purposely designed to create an overall sense of joy with intensity of purpose. Next, I will describe participant recruitment for this study.

\section{Participant recruitment.}

My aim was to select a range of participants with varying experiences in order to develop thick, rich descriptions of the perceptions of highly experienced educators with diverse viewpoints. Using a purposeful selection strategy, I employed the following selection criteria: (a) at least five years of experience working with children between the ages of 2 and 5 years old; (b) currently employed in a licensed, center-based CDC in a 
metro area that has a creative reuse cultural center (CRCC); (c) worked previously in a variety of educational settings (in-home programs, center-based, lab schools; (d) with children of varying ages within the range of 1-to-5 years, (e) identify as a social constructivist; (f) identify as ecologically minded; and (g) have varying years of mature experience teaching in the classroom.

To recruit educators that identify as social constructivist and ecologically minded, I asked candidates over the phone or via email: Are you familiar with Remida Cultural Centers? Do you work with reuse material in the classroom?

This study was informed by Katz' (1995) description of the stages of early childhood educator professional growth patterns and professional development needs. It is important to note that from a post-modern perspective, the concept of development is of doubtful validity and problematic (Burman, 1994; Grieshaber \& Cannella, 2001). Critical theory holds that the whole concept and practice of development reflects Western-Northern hegemony over the rest of the world. According to many Latinx and Chicana/o theorists, the way we understand development is rooted in the earlier colonial discourse that depicts the North as "advanced" and "progressive," and the South as "backward," "degenerate," and "primitive" (Escobar, 2011; Esteva \& Prakash, 1992). However, many early childhood educators believe that the construct of human development is meaningful. They find it useful to think of teachers as having developmental sequences in their professional growth patterns (Katz \& Weir, 1969). 
According to Katz (1995), the average educator reaches a stage of professional maturity after the third (oftentimes after the fourth or fifth) year teaching. According to Katz (1995), at this stage of maturity the educator:

Is likely to have come to terms with herself as a teacher and to have reached a comfortable level of confidence in her own competence. She now has enough perspective to begin to ask deeper and more abstract questions, such as 'What are my historical and philosophical roots? What is the nature of growth and learning? How are educational decisions made? Can schools change societies? Is early childhood teaching really a profession?' Perhaps she has asked these questions before. But with experience, the questions represent a more meaningful search for insight, perspective, and realism. (para. 12, p.4).

To ensure the degree of common ground necessary to foster highly interactive and self-sustaining conversations, I informed potential participants how the flow of the interviews would go and asked whether they were interested in participating in the narrative inquiry before deciding to incorporate them into the study. I informed them that the interview was designed in two parts: (a) through hands-on reuse collage, where they would reflect on their professional experiences, and then (b) share their stories through pedagogical documentation of creative reuse. Participants were encouraged to bring documentation that would make their Remida-inspired identity studies visible. I informed participants I would collect data through a dyadic interview that would involve discussions of their identity as early childhood educators and social constructivists. And use the collage during the beginning of the interviews and documentation during the 
second session of interviews as support. All six of the participants screened agreed they were interested in participating.

The research design called for two pairs of educators to participate in the study. I pre-screened nine potential Remida-inspired participants using phone interviews and decided to keep six from two different schools who self-identified as people who intentionally co-construct social identities with children 2 to 5 years of age. All six of the potential participants screened met these criteria; two of these six were meant to serve as alternates in case one or two members of a pair exited the research project prematurely. One director participant was unable to participate in the focus group, however, her dyadic partner did participate in the focus group and the directors' second dyadic interview was cancelled and was not able to be rescheduled. I chose to keep all six participants' voices in the study (rather than remove all of the data collected from the participants whose participation was limited). There was plenty of data derived from the methods used so that the shifts in participation did not create an effectual loss.

Further, before agreeing to participate, participants were asked to read and sign a Letter of Informed Consent (see Appendix F). This letter described the purpose of the study, the research methods to be used, and possible risks and benefits of participating. The letter confirmed that participation in the study was voluntary and that participants were free to withdraw at any time without repercussion.

\section{Participant description.}

The study participants were all female educators who work in one of two (and sometimes both) local Reggio-inspired preprimary school settings: the laboratory CDC or 
the corporate CDC. Originally, I proposed forming relationship-based dyadic pairs consisting of educators from the laboratory CDC and the IRPP advisory committee preschool. The first pair of acquaintance-based participants recruited and interviewed work as studio teachers at the laboratory CDC and a corporate CDC. The studio teacher's ages ranged from 40 to 50 . They both had experience working in a variety of centerbased and child development laboratory schools and identify as having reached a stage of professional maturity with 20 years of experience in early childhood education and at least 10 years of Reggio and Remida-inspired work.

While transcribing their interview I became inspired to select the rest of the participants from the same two schools and to form a dyadic pair of classroom teachers and a pair of directors from each Remida-inspired learning community. Of the three sets of paired participants, one dyad was relationship-based (long-term association), one dyad were acquaintances (familiar with one another), and one-dyad was stranger-based (did not know each other prior to the study). Regarding strangers versus acquaintances, Morgan (1997) notes, "The rule of thumb favors strangers because, although acquaintances can converse more readily, this is often due to their ability to rely on the kind of taken for granted assumptions that are exactly what the researcher is trying to investigate" (p. 37). These intentional pairings were thought to stimulate a reflective experience that would elicit meaningful data relevant to my research questions.

Once my first acquaintance-based dyadic pair of studio teachers was identified from two schools with a creative reuse cultural center, I formed the second stranger-based dyad comprised of a classroom teacher from the corporate CDC (who was recommended 
by her school's studio teacher) and then a classroom teacher from the laboratory CDC was selected (who sits on the IRPP advisory committee). The classroom teachers ages ranged from 30 to 40, they both had experience working in a variety of Reggio inspired laboratory and CDC's and identify as having reached a stage of professional maturity with 10 years of experience in early childhood education and at least three years being Remida-inspired.

To reiterate, I have given pseudonyms of the "laboratory $\mathrm{CDC}$ " and the "corporate CDC" to protect the confidentiality of the schools and participants. Both are in the Pacific Northwest region of the U.S. and are strongly influenced by the philosophies of the Municipal Preprimary Schools and Infant/Toddler Centers of Reggio Emilia, Italy.

The third dyadic pair was created when the director of the laboratory CDC expressed that she was willing to participate. She then helped to recruit the director from the corporate $\mathrm{CDC}$ with whom she shared a 30-year professional relationship, thus these two formed the relationship-based dyad. The director's ages ranged from 50 to 60 and they both had experience working in a variety of CDCs and identify as having reached a stage of professional maturity with 30 years of experience in early childhood education and at least 10 years being Remida-inspired. I realized at this stage of recruitment that I was including a director pair as educators and that this might pose some challenges to the study as they were not working directly in the classroom. I decided the benefit of having their seasoned and administrative perspectives included in the study would outweigh any disadvantages of their non-involvement in classrooms daily. Both reported strong 
ongoing interactions with children in each center at the time of recruitment as well as both had been longtime classroom educators previously.

I intentionally chose diverse individuals who were involved in classrooms with children between the ages of 2 and 5 years old, worked previously in a variety of settings (public school, center-based, lab schools), and who were of varying ages and have varying years of mature experience teaching in the classroom. Next, I will present the data collection procedures employed in this study.

\section{Data Collection Procedures}

To elicit reflective perceptions among this group of Reggio-inspired early childhood educators, I selected several distinct data collection methods. The following data collection procedures were used: Dyadic interviews and focus group discussion, analytic memos and artifacts collected during the dyadic interviews, and a focus group discussion. As a narrative inquirer, rather than seeing triangulation as a method for validation or verification, I used these distinct data collection methods to ensure that my account was rich, robust, comprehensive, and well-developed.

It was my intention to complete the dyadic interviews and focus group discussion in a strategic order so that data could build on each other. In the first dyadic interview, I introduced a reuse activity where each participant made an impermanent, creative representation of herself using re-purposed materials. During the second dyadic interview, the pairs made an impermanent reuse representation of each other. In both the first and second dyadic interviews, the creative artifacts generated served as a stimulus for discussions related to social identity and pedagogical documentation. Participant's 
responses to these portraits were guided by the questions provided by the researcher.

Each of these conversations was audio recorded.

Data collection Timelines.

The following table presents a timeline of the research:

Table 1

Timeline of Data Collection

\begin{tabular}{|c|c|}
\hline Week of Data Collection: & $\begin{array}{l}\text { Data Collection Procedure(s) to } \\
\text { Complete: }\end{array}$ \\
\hline $\begin{array}{l}\text { Dyad 1: Studio teachers } \\
\text { - } \quad \text { December 14, } 2015 \\
\text { Dyad 2: Directors } \\
\text { - February } 24,2016 \\
\text { Dyad 3: Classroom teachers } \\
\text { - April 30, } 2016\end{array}$ & $\begin{array}{l}\text { Dyad Session \#1 } \\
\text { Artifact collection of impermanent self- } \\
\text { representation with reuse materials. }\end{array}$ \\
\hline $\begin{array}{l}\text { Dyad 1: Studio teachers } \\
\text { - } \quad \text { January 15, } 2016 \\
\text { Dyad 3: Classroom teachers } \\
\text { - May 22, } 2016\end{array}$ & $\begin{array}{l}\text { Dyadic Session } \# 2 \\
\text { Artifact collection of impermanent } \\
\text { representation of other with reuse } \\
\text { materials. } \\
\text { Artifact collection of reuse identity } \\
\text { studies documentation }\end{array}$ \\
\hline $\begin{array}{l}\text { Dyad } 1 \text { and } 3 \text { and one director from Dyad } \\
2\end{array}$ & $\begin{array}{l}\text { Focus Group discussion } \\
\text { Artifact collection of persona dolls made } \\
\text { with reuse materials. }\end{array}$ \\
\hline
\end{tabular}




\begin{tabular}{|c|l|}
\hline & \\
\hline $\begin{array}{c}\text { Dyad 2: Directors } 14,2016 \\
\text { - Canceled }\end{array}$ & Dyadic Session \#2 \\
\hline
\end{tabular}

The actual timeline of data collection did not exactly match the proposed timeline. I finished recruiting my six participants on March 4, 2016, which was five months past my proposed date. Although it took longer than I expected, throughout the process I decided to focus on pairs of studio teachers and classroom educators from two schools and added two director participants.

\section{Data Collection Protocols}

This qualitative study involved six dyadic interviews, two interviews per pair, and one focus group interview involving five of the six participants in the study. Due to a scheduling conflict, one of the participants was unable to attend the focus group discussion, although all six participants were invited to attend. I also collected and investigated eight representational artifacts and four artifacts of documentation generated during the dyadic interviews and single focus group discussion. The treatment of these artifacts and analytic memos is described later in the chapter. The following presents these data collection protocols.

\section{Interviews.}

Qualitative interviewing becomes "a way of finding out what others feel and think about their worlds" (Rubin \& Rubin, 1995, p. 1). In this research, the dyadic interviews provided time and space for participants to articulate their beliefs and thoughts about how 
they came to identify as social constructivists and Reggio-inspired instructors, how they were introduced to Remida reuse education, and their socially constructivist approaches to identity studies using Remida-inspired materials.

Dyadic interviews have been in existence for some time under different labels including joint, peer, paired, or two-person interviews (Morgan, 2016). The appeal of this method over triadic interviews is that "the classic idea of a conversation between two people is unique to dyads" (Morgan, 2016, p. 10). In this study, dyadic interviews created a space for the voices of early childhood educators using alternative and reclaimed materials to emerge. The transformational power of response as paired participants with similar roles as Remida-inspired educators met within the threedimensional narrative inquiry space was illuminated as each narrator was supported in retelling her story in more attentive ways (Clandinin, Huber \& Steeves 2011). Together these two social actors contextualized their temporal, social, and spatial experiences and multiplicity of identities to make sense of what it means to socially construct identities.

I chose to involve both dyadic interviews and a focus group for two reasons. First, conversations between a pair of participants and an interviewer are not the same as the group discussions that take place in focus groups. For example, dyadic interviews provide more time for each person to formulate and share ideas. This time tends to allow for more depth of reflection and detailed descriptions of perceptions as the participants respond to each other (Morgan, 2016). Secondly, while the process of collecting extensive qualitative data such as in a narrative inquiry study is extremely time consuming, I was inspired by Maple and Edwards' (2010) observation that "it is this level 
of engagement with and immersion in the data that led me to understand in a unique way the narratives that I collected" (p. 46). I personally transcribed 167 pages of audiorecorded narrative data: 51 pages of session one dyadic interviews (all three pairs), 53 pages of session two dyadic interviews (studio and classroom teacher pairs) and 61 pages of focus group discussion. This degree of engagement and immersion in the data led me to understand the narratives that I collected in the unique way that reflection on that level affords.

After comparing focus groups and dyadic interviews, Morgan (2016) observed that both produce similar content with the same topics appearing in each and receiving similar amounts of emphasis. Where the differences occur is in the dynamics of the interaction. For example, in focus group discussions, each turn at talk tends to be longer and more self-contained, while the exchanges in the dyadic interviews are more rapid and intertwined. As a relatively new research method, there are many other creative uses of dyadic interviews and focus groups that have yet to be discovered.

In this study, I have engaged a multi-method use of dyadic interviews and a focus group discussion with a pool of six participants in an authentic partnership - matching the goals of my research with the data that the dyadic and focus group could produce through an emphasis on research design. I intentionally used paired conversations between Remida-inspired educators who share the same roles to produce narrative data and insights that would be less accessible without the interaction found in a dyad and then compared the data with the narrative inquiry that would be less accessible without the 
interactions found in a group discussion of experiences with reuse materials in Reggio Emilia inspired identity studies.

Dyadic interviews. Several tools were employed to support the participants' reflective experiences in both the dyadic interviews and the focus group discussion. I begin with a description of the dyadic interviews and the tools used to elicit participants' perceptions. To understand early childhood educators' experiences with reuse materials in Reggio Emilia inspired identity studies, I completed two dyad interviews with each participant during the study. Again, I recruited a standby dyad pair to alternate should one or both of an existing pair exit the study prematurely, but I ultimately decided to retain all six participants and hold three sets of two interviews per dyad. The intention of the dyadic interviews was to create a space for participants to actively work with reuse materials and simultaneously reflect on their experiences with toddler-age and preschool children's creative reuse and to articulate their underlying belief structures about identity, cognition, and reuse.

The dyad interviews use an Interview Guide (see Appendix E) that asked the following general questions about participants' experiences and perspectives working with reuse materials in early childhood identity studies. Nine open-ended questions were asked during two semi-structured dyadic interviews and three open-ended questions were asked during the semi-structured focus group.

The initial dyad interviews primarily focused on these early childhood educators' perceptions related to social constructivism and Reggio-inspired practices in their reuse practices. The following ice breaker and questions were posed (to each of the three 
dyadic pairs) to guide the first dyadic semi-structured interview: What was it like to represent yourself with reuse materials?

- Please share with one another the story of how you came to identify as a social constructivist and Reggio-inspired early childhood educator?

1. How did you come to be Remida-inspired?

2. What does identity studies in early childhood education mean to you and how have you approached identity studies with young children as a social constructivist educator?

3. How did visiting Reggio Emilia Remida impress you?

The second dyad interview asked participants to articulate their experiences and perspectives about documenting identity studies and working with Remida-inspired materials with children. Additionally, I asked participants to describe how their identity studies with young children have evolved over time and how Remida-inspired materials have enriched or challenged their approach to engaging children in identity studies.

Further, because educators work within the context of a school environment with families, students, administrators, and often co-teachers, I posed questions that sought to uncover how educators engage in creative reuse cultural education with the families and co-teachers that make up their learning communities. For example, during the second session of dyadic interviews with studio and classroom teachers I asked questions such as: "What is it like to work with a co-teacher and families when socially constructing identities with children using reuse material?" By asking these questions, I posit that I developed a deeper understanding of everyone's experiences within the broader 
ecological system of their learning communities. The final question was originally designed to be a follow-up email prompt. Due to a lack of participant response to this email prompt, I added the second email prompt to the second dyadic interview questions. First, the participants reflected on the ice breaker collage provocation: What was it like to represent your pair?

- What does documentation mean to you? Please share your documentation of reuse and identity studies.

1. Can you share stories with one other about your experiences with reuse and children and what is it like to work with a co-teacher, and families when you co-construct social identities with your students using reuse materials?

2. What is difficult, challenging or rewarding about working with reuse materials with children and how does that affect you?

3. Do you identify as an atelierista? What is your image of the teacher?

Dyadic interviews were conducted in the IRPP center (located at the laboratory CDC) at a date and time chosen by participants. Each interview lasted 45-90 minutes. Participants were invited for a two-hour time slot to allow for an additional half hour for arrival, instructions, and informed consent forms to be explained and filled out. With consent, all interviews were audiotaped so that I could engage as moderator, take notes, encourage free-flowing discussions, and support a high level of mutual engagement between the participants.

As it turned out, my presence during the first two studio teachers' interviews and the first director dyadic interviews was awkward because participants attempted to 
include me in their conversations. As a result, approximately 15 minutes into each discussion, I left the room for the remainder of the classroom teachers' first and second dyadic interviews. This generated mixed results: on the one hand the interviews flowed more easily without my presence and on the other hand I was not there to redirect the conversation when one of the participants failed to share her experience in response to a question. The audio recorder was on for all the conversations between participants during creative reuse representation provocations except for the first director dyad. I intentionally did not record the directors' conversation during their hands-on reuse provocation because the pair of long-time colleagues appeared to desire time to catch up in private while engaging in the provocation. When I suggested I would initially give them privacy and not record, they both seemed appreciative.

To ensure the confidentiality of the participants and security of the audiotapes, the digital recordings will be stored on my password-protected secure computer and external hard drive until the appropriate three years wait time (per IRB) has passed and the recordings will be destroyed. After transcribing each interview, I saved the corresponding audio file as an individual electronic file on my personal computer and backed up the file on an external hard drive that I also keep in a locked filing cabinet, to ensure no files are accidentally lost until they will be destroyed in 2020 .

After each audio-recorded interview, I planned to create an Interview Cover Sheet, listing pertinent information such as the date, time, and location of the interview as well as the interviewee's pseudonym. Instead, I kept track of this information in a 
research journal. I also typed my initial thoughts and questions about the interview onto the margins of transcripts while listening to the recordings.

\section{Reuse representation artifacts.}

Before each dyadic interview and the final focus group discussion, I invited participants to look at the materials. For the base of their representational collages, I had several pieces of felt ovals in different shades of skin tone that they could use. For the collages and persona dolls, they could use any of the material provided. There were natural and found objects, manufacturer discards, and scissors. So, in collage, they could look at materials and think about what materials would help them in making a symbolic and or literal representation of themselves in the first session, of their dyadic partner in the second session, and of the persona dolls in the third session. One advantage of collage is that the participants could move objects around. So, all the materials could come together, and they could organize them in a way they found represented themselves, their pair, or the persona doll. On the table, I left a piece of paper with the prompts about identity and reuse. Once they were ready to start, I left the room. The digital recorders captured their conversation in my absence. The pairs took as much time as they wished. I was outside the room if they had any questions. When they were ready, they were instructed to come get me to begin the interview. Participants then worked on the hands-on creative reuse provocations.

\section{Documentation artifacts.}

Participants were asked to bring relevant artifacts to the second dyadic interviews such as classroom documentation; classroom curricula; and email exchanges with 
administrators or families; photographs; or videos. This invitation offered interviewees another way to reflectively share their experiences and perspectives about socially constructing identities with young children using reuse materials. About reflecting on documentation as a form of professional formation, Rubizzi (2001) wrote, “Our hypothesis is that when teachers reflect on and discuss their choices and their actions, their awareness of the proposals they make to the children is significantly heightened" (Project Zero \& Reggio Children, 2001, p. 94). Using documentation artifacts as a stimuli was intended to focus the mind and elicit situated cognition experiences by stimulating very relevant and genuine conversations about educators' experiences of working in a Remida-inspired way around identity studies.

Again, I collected and investigated eight representational artifacts, four artifacts of documentation, and two reuse persona doll artifacts generated during the dyadic interviews and focus group discussion. These artifacts were also used as sources of data for my research. I promptly and systematically recorded the artifacts by completing an Artifact Cover Sheet which was stored with a photograph of the reuse artifacts or a copy of the documentation artifact before filing them into a locked filing cabinet. The artifact cover sheet was used to document the date and time the artifact was received, the participant who shared the artifact, and a description of the artifact such as if it was a classroom document, classroom curricula, photograph, video, or email exchange with administrators or families,. 


\section{Focus group discussion.}

Research related to focus group design (e.g. Morgan, 1997) has suggested that it is important to maintain a relatively tight focus and not attempt to explore too many topics during one discussion. Therefore, for my focus interview design, I chose to pose the prompt: "Create a Remida-inspired persona doll using reuse materials and make a story about the doll by choosing to answer the following questions about the doll." The focus group separated into two groups of members from each school working together and then rejoined into one group for the final reflection. The two groups were given identity planning charts from which to select characteristics of the dolls to represent with reuse materials and data collected from dyadic interviews to choose from to collectively answer the following questions based on participant's descriptions of themselves and each other during reuse representational portrait provocations.

1. How did the doll come to identify as a social constructivist and Reggio inspired early childhood educator?

2. What does identity studies in early childhood education mean to the doll? How has the doll approached identity studies with young children as a social constructivist?

Open-ended questions, brainstorming sessions, and persona dolls completed during the focus interview produced entirely new question formats for future research. For instance, in such meetings while involving hands-on exploration of materials in a focus group, this kind of immersion in the experience contributed to engaging educators' imagination and creativity even more in a learning experience; an experience that can 
foster and develop ideas and new ways to organize the ecological and anti-bias learning contexts and subject matters to be offered to the children in their pedagogic experience (Remida Series, 2016). In such meetings, the focus group took a multi-metaphorical approach to pedagogical notions such as how we ask children, teachers, and families to identify with reuse materials through three-dimensional social constructions of persona dolls that would personify their ideal of sustainability and reuse educational practices as an embodiment in the doll.

One doll named "Logan" chose to use gender-neutral pronouns for themselves and has a persona of gender fluidity. This doll has traveled to several schools introducing the doll to classrooms of children and opening the community to their ways of living and experiencing the world around many topics involving ecology, culture, and learning (W. Parnell, Personal Communication, June 25, 2017). More information on this research finding will be explored in Chapters 4 and 5.

\section{Email Prompts.}

To gain a deeper understanding of how participants think about and engage with ecological identity studies using reuse materials, I intended to use email prompts as a data collection strategy. Using both interviews and email prompts as data collection procedures was envisioned to accommodate the potentially diverse communication styles of the participants. I find that people often feel that they communicate better when either speaking or writing. By conducting both oral interviews and requesting written responses to email prompts, I was hoping participants would feel that they were being provided with at least one communication vehicle that they were comfortable with to express their 
thoughts and feelings effectively. For these reasons, participants were asked to respond to one of two email prompts throughout the study. The participants were reminded that the questions were intended to gain a deeper understanding of participants' beliefs concerning socially constructing identities and that responding to the email prompts was a mandatory part of participating in the study, should they choose to proceed. They were asked to spend 10- to 20-minutes responding to each email. The email prompts had two questions (see Appendix E) that I asked of all participants. The first and second email prompt asked:

1. How did visiting Remida in Italy impress you?

2. Do you identify as an atelierista? What is your image of the teacher?

As it turned out, the participants chose not to respond via email and so the questions were woven into the dyadic interviews. This had the effect of creating longer interviews. However, although the participants appeared to be tired by the end of the dyadic interviews, their immersion experience generated the rich responses shared in Chapter 4.

I initially intended to send a second email prompt at the very end of the study that was going to ask the participants to reflect on their experiences thinking and talking about young children's identities throughout the study. This second email prompt was meant to offer participants an opportunity to reflect on specific components of data I had collected from them earlier. These questions originally designed for this second email were (a) We have now had many different conversations (through dyad interview, artifact description, and email) about young children's social identities, is there anything else you would like 
me to know about your experiences and perspectives related to this topic? And (b) after thinking so deeply about young children's social identities, are you left with any big questions or ideas about early childhood education for sustainability? However, because the participants did not respond via email or in hand-written responses, the second email prompt was abandoned altogether.

I originally intended to collect data from follow up interview questions via email but since neither the first nor second pairs of participants responded to that prompt after their first dyadic interviews. I wove the additional questions into the second dyadic interviews. Additionally, I integrated the first email prompt into the first dyadic interview session with the classroom teachers who were scheduled after the first two pairs.

\section{Data Interpretations}

Again, this study aimed to illuminate Reggio-inspired early childhood educator's experiences and perspectives about socially constructing identities with young children using Remida-inspired materials. Towards this end, I collected and interpreted data iteratively so that my research procedures (the questions I asked during dyadic interviews and focus group discussions and analysis of the artifacts) could be informed by the data as they were gathered (Lichtman, 2013). As a qualitative researcher, I used an inductive strategy by asking open-ended questions using artifacts as a stimulus. My aim in being inductive was to make meaning of experiences in an inviting setting and to elicit the perceptions of those being interviewed. 
The qualitative narrative inquiry data collected through dyadic interviews and focus group discussions were interpreted using the reflective process explained below. Although many narrative researchers have chosen to write about themes and concepts derived from the data through thematic analysis, I used stories to convey meaning through narrative representation. Rather than coding and determining themes by making discretionary determinations, I focused on the stories and narratives of my research participants holistically. I analyzed the data sets (through interview transcripts, analytic memos, and artifacts) and then unified them by representing them in the form or shape of a narrative story. The created story may be seen as a narrative representation of thinking with identity through reuse materials. Here, the creation of the story itself is considered an act of narrative analysis (Clandinin, 2007, p. xv).

In the later stages of interpretation, I engaged in metaphorical interpretation of the narratives the participants and artifacts communicated (Patton, 2002). I explored various metaphors and how well they fit with what emerged in the narrative data collection. I asked participants for metaphors and listened for spontaneous metaphors during dyadic interviews and then I checked for the validity of those metaphors with research participants during the final focus group.

A systematic approach to interpretation brought order and understanding to my narrative inquiry. I exercised creativity and discipline as I embarked on my data interpretation. In other words, the challenge was to remain flexible and open to discussion and interpretation while simultaneously finding order in the data sets. One way that I accomplished this was to collect data at intervals across the life of my research 
project. This tiered approach of data collection and analysis provided the opportunity to first interpret how the participates related the creative reuse prompts to their own identity as educators, and then to interpret how the participants engaged with materials to conceptualize social identity construction with children. I concluded with a final focus group and dyadic interview that provided a member check (Lichtman, 2013) to ensure that I had accurately interpreted and represented the participants' meaning throughout the narrative inquiry process.

\section{Re-storying.}

Maple and Edwards (2010) claimed that "metaphors and other visualization techniques can enable you to examine the data from a range of perspectives" (p. 47). They noted that there is no distinct formula for how to conduct narrative inquiry interpretations because unlike other step-by-step qualitative methods of interpretations, narrative inquiry looks to understand the meanings found within the story. This lack of formal analytic steps has the advantage of allowing for freedom and flexibility of data interpretation that is unique to this method (Maple \& Edwards, 2010).

Maple and Edwards (2010) recommended using metaphors to explore the plot of a participant's story and unlock data. They suggested that "Metaphors provide a mechanism to relate, compare, and make meaning of new knowledge with lived experiences" (p. 40). In keeping with these recommendations as a social constructivist project, a vital element of constructing my interpretations was to explore and make sense of the data from a range of perspectives. This was facilitated by the use and creation of metaphors as well as through dialogue with my participants to co-construct meaning and 
build new knowledge as Reggio and Remida-inspired investigative partners and collaborators. Throughout, in keeping with a Chicana studies narrative inquiry approach (Galindo, 2007), or auto-historia teoria (Keating, 2016), I intentionally avoided mining the data for themes because of the risk of depersonalizing and decontextualizing the stories of each participant. Rather, I looked for similarities among potentially emergent narrative patterns through metaphoric meaning.

Patton (2002) noted, "Metaphors and analogies can enliven and enrich descriptions, helping readers connect through shared understandings and giving them a better feel for the environment being described" (p. 281). I engaged participant's metaphors as well as searched for my own to interpret the data to re-tell a story manifested through the processes of data collection, analysis, and interpretation. As Lichtman (2013) suggested,

The intention is to examine how such stories can be used as structured or formal ways to transmit information (Connelly and Clandinin, 1990). The specific details of how I moved from the raw data into a narrative or story mode is based more overall on the data rather than isolating each part into specific codes. In a sense, my process is about the whole rather than a focus on the details as I concentrated on the gathered narratives and look for epiphanies. The re-storying process involves collecting and rewriting the participant's stories to place them within a plot that incorporates a main character who experiences a conflict or struggle that comes to some sort of resolution. (p. 256) 
Lichtman's approach offers an alternative to conventional data analysis. These ideas inspired me to consider how re-storying is useful to interpreting narrative data based on a social constructivist perspective that employs successive writing and rewriting and discussing stories. This work promotes conceptual application and critical thinking with the power of creative reuse activities in the context of identity studies. As a result, I adopted the re-storying approach because this interpretive and creative method invites the reader to learn new concepts and new perspectives related to familiar ideas through storytelling.

Here is where the teacher-as-researcher aspect of this study bears responsibility. The sustained interpretation of past personal experiences may result in deeper internalization of Remida principles for the researcher, participants, and reader. A restorying approach invites one to consider how personal and educator-generated stories about reuse and identity studies may be practiced, shared, reflected upon, revised, and expanded upon in future Remida-inspired early childhood education for sustainability research.

I see data interpretation as a process of sorting and sifting similar perceptions and experiences to the process of organizing materials in our creative reuse cultural center. Organizing the data collected took on the same kind of challenge and exchange of ideas as hosting, introducing, displaying, exhibiting, and offering repurposed materials. There were no clear bounded rules dictating my process. As a beginning co-researcher, I learned by doing. By following an iterative process, I could begin to make sense of my data by deconstructing and then socially constructing and re-unifying through re- 
presenting emergent narrative themes or patterns and giving them new life in the form or shape of a metaphorical narrative story.

\section{Analytic memos.}

Throughout my interpretations I completed analytic memos that were like research journal entries wherein I wrote down my thoughts about my data. The purpose of completing analytic memos during my data interpretations was to reflect deeply on the research process itself on an on-going basis throughout the data collection and analysis cycle (Chang, 2008). For example, through writing analytic memos, I reflected critically on my role as a researcher and how my assumptions, thoughts, and beliefs influenced my research techniques and focus (Chang, 2008). This procedure supported me in my process of recognizing that all interpretation is subjective and that I bring my personal experience and theoretical lens to the narrative inquiry analyzing processes as well. That said, analytic memos aided my process of recognizing my own subjectivities and deeply considering what the participants meant. Hence, my subjectivity is not "reduced" but rather enhances the work I am engaging.

Throughout the data interpretation process I used the margins of transcripts to record self-observations: my own thoughts, reflections, and notes about the data. This allowed me to be actively thinking with and engaging with the data throughout the study (Chang, 2008). I also drew from van Manen's (1990) approach to qualitative research methodology in education that is distinct from traditional approaches derived from the behavioral or natural sciences. Rather than relying on abstract generalizations and theories, van Manen offers an alternative that taps the unique nature of each human 
situation. This is an approach rooted in the "everyday lived experience" (p. 35) of human beings in educational situations.

An example of van Manen's hermeneutic-phenomenological inquiry is his metaphor of knots in the narrative, "Knots in the webs of our experiences, around which certain lived experiences are spun and thus lived through as meaningful wholes" (van Manen, 1990, p. 90). As I analyzed my data, although each participant's experiences were unique, patterns came into view which I conceptualized through three archetypal stages of becoming Remida-inspired: The Forager, The Global Homesteader and The Navigator. I was inspired by van Manen's (1990) use of the metaphor of a conceptual knot for how to orient oneself to human experience in education and emphasized the role of language in educational research, and the importance of pursuing social science research critically as a semiotic writing practice.

Using an analytic memo approach, I engaged a narrative mode of writing and analyzing data to locate and understand the voices of the research participants, as well as my own. I was inspired by how Maple and Edwards (2010) illustrated, The absence of a linear path in narrative inquiry, and highlight an interactive, engaging, one-step-forward, two-steps-back kind of journey. This approach to writing not only represents a way to analyze the data, but also demonstrates how narratives can inform the work in a way that honors the whole (p. 33).

By engaging participants in semi-structured discussions loosely guided by open-ended prompts and using metaphor and narrative themes to interpret data, my aim was to honor the meaningful whole of early childhood educators' experiences with reuse materials in 
Reggio Emilia inspired identity studies. Clearly, I did not attempt to conduct exhaustive research to learn everything my participants experienced related to the research question as the phrase "honors the whole" implies. Rather, my aim was to demonstrate how narratives can inform the work in a holistic way. For example, the stimulus I provided inspired my participants to share various aspects of their perceptions from different perspectives. Next, I will deepen my statement of positionality.

\section{Additional Thoughts About the Research Process}

Complete objectivity is not possible, nor is it desired within a study rooted in a social constructivist framework. Who I was as a Remida-inspired program coordinator and experienced educator affected the study itself. My multiple identities derived from personal and educational experiences influenced how I viewed and conducted the research study, the questions I asked, and the analytical categories I created. Thus, it is my intention to be transparent about who I am and the impression I left on the study.

I am a first-generation Anglo-Mexican, polytheistic, working class, cis female in my late forties. I possess a Bachelor of Social Work degree, a Post-baccalaureate in Chicano/Latino Studies, and a master's degree in Education with a specialization in Postsecondary, Adult, Continuing Education and Education for Sustainability. I have spent many years analyzing and identifying the unearned privileges I receive as a White person with higher-education degrees.

As a teacher researcher, I am drawn to conceptual and theoretical issues that inform critical perspectives of childhood. My doctoral curriculum and instruction specialization focused on issues of teaching and learning related to sustainability in 
Reggio Emilia inspired Pre-K settings. There are currently 17 Remida centers in the world, and I was fortunate to be a coordinator with the IRPP center (2014-2018), which is housed on a university campus in the Reggio-inspired center where the interviews and focus group took place.

I acknowledge that studio teachers, classroom teachers and center directors have different roles and therefore have diverse experiences with reuse materials in Reggio Emilia inspired identity studies. I have not walked in the shoes of any of my participants. I come to my research aware that my lived experience may be, and probably is, very different than the participants of the study. As with all my work, I hope to learn from, and alongside, the individuals agreeing to be a part of this research study.

This is a dissertation aimed at developing new knowledge; a new understanding of how educators perceive that involving children in creative experiences involving reuse materials affects the construction of their social identities. My graduate specialization is in constructivism in early childhood education and is grounded in the works of Jean Piaget and Lev Vygotsky as well as influenced by the infant and toddler centers and preprimary schools of Reggio Emilia, Italy. As a constructivist early childhood educator and researcher, I continually observe how the interactions that young children have with the world around them are critical in shaping their identity and cognition. As a social constructivist, I recognize that my subjectivity shapes my critical theory and that my interpretations flow from personal, cultural, and historical experiences. Grounded in my subjective experiences as an Anglo Chicana and as an adult third culture kid (ACK) I 
readily embrace post-modernist conceptions of individual and social identities as fluid, multidimensional, intersecting and mutually interdependent.

My theoretical approach to studying self-concept and cognitive processes in young children is constructivist and is rooted in my post-Bachelor and graduate research in bridging ecology, culture and learning through the conceptual frameworks of Chicana/o studies. My educational background, along with my work experience, has informed my belief that the social construction of identities begins in early childhood.

A Chicana studies perspective calls on a researcher to acknowledge the power relationships between researcher and participant within the research process (Poniatowska, 1969). My visible identities influence the way participants perceive me and influence the ways in which we interact and the conversations we have. Rather than denying that who I am influences my research, I was explicitly as transparent as possible about my position and role as a researcher (Castillo, 1994) when recruiting and working with my participants. For example, I repeatedly affirmed that I cared deeply about the participants' experiences and voices, that their perceptions were uniquely valuable, and that I wanted to learn from them.

I also strived to reduce the influence that my presence had on the dyadic interviews by leaving room for them to ask a lot of questions and by being straightforward about my intentions to create a nonjudgmental and open atmosphere between us. I clarified my role by saying that "As a moderator, I am not a group leader and I am here because I want to learn about your perspectives." In fact, even my role as moderator was downplayed stepping out of the dyadic interviews at certain points to 
encourage free-flowing exchanges of ideas between the two participants and by actively encouraging a high level of engagement between the focus group interviewees.

\section{Summary}

The purpose of this dissertation was to conduct narrative inquiry research on Reggio-inspired early childhood educator's experiences of socially constructing identities with children using reuse materials. There is a lack of literature connecting children's social identity constructions and early childhood education for sustainability (ECEFS). This gap in literature can be attributed to a common misconception that young children are not able to understand and express more complex social identity nor identify as global citizens.

Overall, developmental psychology has been primarily concerned with the initial emergence of the self-concept rather than the co-construction of identities in ECE. Early childhood developmental research directed at the personal self is problematic from a social identity approach whose orientation is on the development of the social self. In this study, I explored the new and evolving epistemological spaces created by Remidainspired educators, outside of the context of Reggio Emilia, Italy. My main research question is: What are early childhood educators' experiences with reuse materials in Reggio Emilia inspired identity studies?

I interviewed six Remida-inspired early childhood educators from two CDC's located in the Pacific Northwest region of the U.S. about their social constructivist approaches with identity studies, and creative reuse cultural education. For the first session of dyadic interviews, the participants were matched up in pairs of studio teachers, 
directors, and classroom teachers. For the second session of dyadic interviews, the participants were matched up in two pairs of studio and classroom teachers. All of the participants minus one of the directors participated in a final focus group discussion and hands-on reuse provocation. I used narrative analysis, re-storying and metaphorical interpretation analytic approach to interpret my data. These sessions include making reuse collages of themselves and each other, stories they shared about their professional identity formation and Remida-inspired documentation, concluding with narrative data discussions that highlight patterns, metaphors and hidden treasures that were made visible during the interviews. In narrative inquiry session three, I focus on the participants collective experience of making two reuse persona dolls named "Harmony in Disarray" and "Logan" in a focus group, followed by a discussion section examining the possibilities of enriching early childhood anti-bias education through reuse materials as explored through the social construction of persona dolls.

Now that we have looked at narrative research design and methodology, we turn to Chapter 4 interpretation of findings. This chapter creates a space for the voices of six early childhood educators who use alternative and reclaimed materials and is aimed at developing new knowledge - a new understanding of how educators perceive that involving children in creative experiences involving reuse materials affects the construction of their social identities. 


\section{Chapter 4: Foragers, Homesteaders, Navigators}

The problem that this study addresses is that developmental psychology has been primarily concerned with the initial emergence of the self-concept rather than the social construction of identity in early childhood education (ECE). Much of the existing research has either focused primarily on the formation of children's individual identities or has not differentiated between children's personal and social identities (Raburu, 2015).

Using narrative research methodology, the following data informs the research question: What are early childhood educators' experiences with reuse materials in Reggio Emilia inspired identity studies? In considering the value of engaging with objects and materials, Dewey (1938) advocated situated approaches to learning, arguing that developing understanding occurs within social experiences. As this is a social constructivist project, a vital element of this study was to examine a range of professional educator's narratives within the context of social experiences. I elicited experience from educators who serve in a variety of roles within two different Remida- inspired early childhood centers to examine their perceptions of facilitating children's work through manipulation of, and reflections with, discard materials to foster social identity studies within a Reggio inspired context.

In chapter 4, I present data that participants shared over the course of 5 dyadic interview sessions. There were three groups of two at each dyadic session followed by a focus group discussion involving all participants - for a total of six interviews. I also collected (a) collages made with recyclable materials and from questions framing the collage-making experiences, and (b) related documentation artifacts brought forth from 
the educators, and 3) persona dolls fashioned with Remida materials. These collections aid in the narrative experience participants had together. At each turn, I highlight metaphors participants used to describe their experiences, revealing theories of identity studies and reuse materials work brought forward by the participants. At the end of each session, I use a re-storying (Lichtman, 2013) method to fashion a collective voice made of all the participants. These re-stories are meant to express the interpretations I see of the multiple perspectives.

Narrative interviews are a methodology that yields data that are both unique-tothe-individual and universal-for-the-individual as in the common motifs and archetypes experienced (Clandinin, 2007). In keeping with Clandinin's ideas, the crux of participant's responses to the main research question and prompts revolve around multiple metaphors they engaged to describe their experiences. Maple and Edwards (2010) recommend using metaphors to explore the plot of a participant's story and to unlock data. Consequently, in Chapter 4, the data is organized around metaphors that participants used in their narratives.

Although each participant's experiences were unique, patterns came into view that I conceptualized through Katz (1972) framework for developmental stages of preschool teachers; survival, consolidation, renewal, and maturity. Also drawing on a Jungian (1969) perspective, I further characterized participants growth patterns with what I interpret as three corresponding archetypal stages of becoming a Remida-inspired educator: The Forager, The Global Homesteader, and The Navigator. In Jungian psychology, an archetype is an inherited pattern of thought or symbolic image that is 
gleaned from the past collective experience of human cultures and is present in the unconscious of the individual.

Data were collected and interpreted simultaneously so that my research procedures (the questions I ask during dyad interviews and focus group discussion) could be informed by the data as they were gathered (Lichtman, 2013). As a result of this process, at times I interpret in the data presentation sections, as a way to precursor what is to come in the interpretation sections.

I begin with a presentation on the first dyad session. Here, I introduce data collected in session one. I share the data in three sections using metaphors to group the data. The three sessions are (a) Identifying with Reggio: The Foragers, (b) Identifying with Remida: The Global Homesteaders, and (c) Socially Constructing Identity Studies: The Navigator.

\section{Session One Dyadic Interviews}

I facilitated the first narrative sessions on both weekday and weekend afternoons throughout the winter and spring for three dyadic pairs from two separate Reggio Emilia inspired CDC's located in Oregon. Again, for this study, the term dyad refers to two people. I called participants together in a Remida-inspired room at the Inventing Remida Portland Project (IRPP) center. During each dyadic session, the participating pair sat at a table in comfortable cushioned chairs in a room called the Identity Atelier that is filled with reuse materials that lend themselves to identity studies. There were organic snacks and tap water available at a nearby table and pairs were each provided with a page listing a series of questions for each session (see Appendix E). During the interviews, I came 
and went from the room where I left an audio recorder and was also listening in on the conversation from the next room.

In the spirit of situated approaches to learning, participants were provided with carts exhibiting a menagerie of colorful and provocative discards. I invited each pair to use these human-made and natural materials to creatively represent themselves. Inspired by anti-bias education, these materials included felt ovals in different skin tone shades to aid in the discussions of identity construction. Written prompts were placed on the table during the first session to guide the icebreaker for the discussion. Examples of these prompts include:

- Represent yourselves using materials from the center.

- Share what it was like to represent yourself with reuse materials.

The resulting self-representations are pictured in Figure 1.

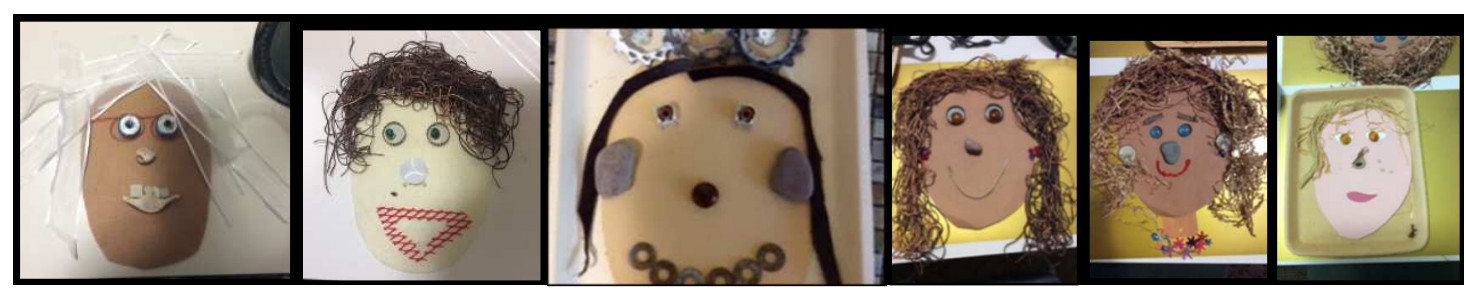

Figure 1. Ann, Maggie, Miranda, Clara, Joni, and Julienne's reuse self-representations.

\section{The first narrative inquiry prompt: The Forager.}

The first prompt was intended to elicit responses to better understand participant's experiences through exploring their pedagogical roots in the Reggio Emilia approach. Beck, Freese, and Kosnik (2004) noted, "As educational researchers and reformers, in our ongoing search for ways to improve teaching and learning, it is important to look backward to take stock of our beginnings, where we have been, what we have 
accomplished, and what we have learned" (p. 20). The prompt: "Please share with one another the story of how you came to identify as a social constructivist, and Reggioinspired early childhood educator," encouraged participants to look backward and take stock of their beginnings. Reflecting on the narrative data that was generated led me to see patterns of professional formation in becoming Reggio inspired. The intention in the first session of dyadic interviews was to create an opportunity for participants to deeply consider their thoughts and feelings about their experiences with creative reuse cultural education, and to begin to articulate their underlying belief structures about working with reuse materials in Reggio-inspired identity studies.

Participants used multiple metaphors to describe their experiences of becoming Reggio inspired educators. The metaphors included: strongest journey, dig into, blossoms, map all laid out, and feeding off. These metaphors inspired me to interpret their first stages of professional formation through a Forager archetype. An overarching pattern that revealed itself across participants' narratives in session one was relating back to their early professional lives as determinant of how they experience reuse materials in Reggio Emilia inspired identity studies. After the interviews were shared, I interpreted the data through forager lenses such as gathering pedagogies; relationality as an adaptive strategy; in search of theoretical frameworks; and sustaining situated learning. Next, I show some of the ways the forager metaphor shows up in the data.

Studio Teacher Dyad. The first interview was with an acquaintance-based pair of studio teachers: Ann from the laboratory Child Development Center (CDC) and Maggie from the corporate CDC. The three of us met at the IRPP center on a weekday in December 
and the interview began with the studio teachers making self-representations with reuse materials as an icebreaker (see Figure 2). As the conversation begins and a few ideas are exchanged along with glances in each other's direction while working, the two educators stopped to reflect/converse. Maggie noted, "Children usually fixate on their nostrils and they often choose two circular materials for their nose; I had to make sure I incorporated nostrils." To this Ann replied, "Children seem to fixate on their nostrils when they look in the mirror. I find it interesting that we both integrated that into our self-portraits, even though we didn't talk about it."

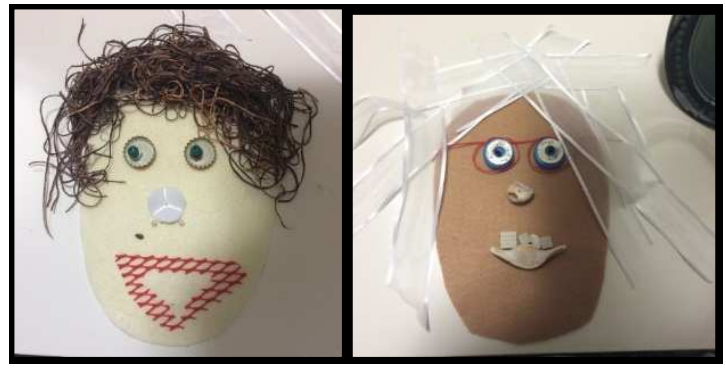

Figure 2. Maggie reuse self-representation (left) and Ann (right).

As the conversation continued, the pair shared with each other how they came to identify as Reggio inspired. They look over each other's work and exchange a few more ideas and Maggie said,

I identified myself as Reggio inspired because I went after it through trainings and the types of places and people that I chose to work with. And then over the years as I was doing more writing and presenting, I realized there is more of a language to how I would describe my teaching practice. 
Ann: I knew that I didn't want to base my curriculum on the weekly scholastic reader and the ditto sheets that people were using. Even though I taught thematic wise, like most people did then in this area, a balance of starting an idea as a teacher and then having children bring in their ideas seemed to naturally come, because it made sense to me, even though I didn't know why.

As the dyad interviews continued, a forager lens emerged. During the interview Ann shared that, "The last 10 years of my teaching have been my strongest journey into what Reggio inspired work looks like, because I had not done that previously. [Prior to that time] I had only experienced the bubbling of what constructivist work could be and look like." Ann used the metaphor of the "strongest journey" to describe her last 10 years of teaching as a studio teacher compared to her earlier pedagogical experiences of foraging for social constructivism in her practice.

Maggie used a metaphor "dig in" to describe her director's encouragement to learn more about the Reggio Emilia system of education. This metaphor also evokes forager. Maggie attributed this enriched experience of foraging to an increasingly wider area of connections through Reggio inspired conferences, literature, and mentorship. She described her networking with others as sustenance for her practice.

Both studio teachers agreed that the last 10 of their 20 years of professional formation have been the strongest periods of their careers as educators. Maggie used the metaphor of blossoms to further describe her vital experience of rich sources of relationship-based Reggio inspiration in her environment as she states: 
Part of that is the growing community; there are so many more people to talk to now, share ideas, and collaborate with that it blossoms. And you don't feel like that weird teacher in the classroom who people are like, what are you doing in your room?

Maggie goes on to use the metaphor of "having the map all laid out" to describe her cognitive experience of transitioning to a Reggio inspired culture early on in her practice. I thought a social constructivist curriculum was going to be hard work, because I had the map all laid out; the curriculum was the same year-after-year at my first school. But doing the same thing is exhausting. It wears you down because it is repetitive and lacks the energy and inspiration of when it is new. You start to become that teacher who asks, what color is that? Even though you know it is blue. Instead, in a Reggio inspired setting you are excited, you wonder, and it is much more fun.

Maggie and Anne's stories related finding professional nourishment and resilience in identifying as Reggio-inspired educators. With the first dyadic interview completed, I note my energy and excitement for wanting to know more. I turn to the narrative session that followed with the Director dyad.

Director Dyad. As a part of the first narrative session, this second dyadic interview was with a relationship-based pair of CDC directors; Joni from the laboratory CDC and Julienne from the corporate CDC. I met with the two Remida-inspired directors at the IRPP center on a weekday in February. The directors shared their experience with one another as they embarked on a search (foraged) for discard materials: 
Joni: I was going to offer you those materials for your eyes, but then I thought, no, I will let Julienne find it. I usually start with my features that stand out. I have a lot of hair, so I got my hair kind of curly and wild looking. I almost always make myself smiling. I am generally a happy person and smile. "Put on that smile!" [Laughter]

Julienne: I was thinking about texture, I was thinking we can make our faces all, you know how you get those wrinkles? [Laughing]

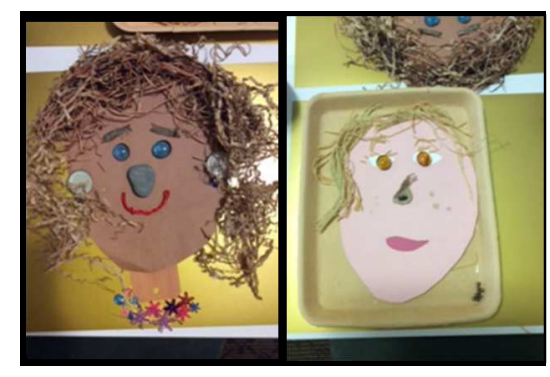

Figure 3. Joni reuse self-representation (left) and Julienne's reuse self-representation (right).

After making self-representations with reuse materials as a warm-up (see Figure 3), the pair - 30-year friends and colleagues — shared with each other how they came to identify as Reggio inspired. I sat in a corner of the identity atelier and took notes during this entire session. The pair considered the first narrative inquiry prompt intended to elicit responses to better understand their pedagogical roots in the Reggio Emilia approach (prompt: Please share with one another the story of how you came to identify as a social constructivist, and Reggio- inspired early childhood educator). Their time together offered the following: 
Joni: [Laughing] Wow! That's a big question!

Michelle: It involves a bit of a story maybe?

Joni: Yeah.

Julienne: I can take a stab at it. There's lots of stories. Social constructivism was always in me, whereas how you work it as a teacher or as a professional wasn't always clear. The idea that you are collecting ideas, that you need to be in relationship with other people to figure out who you are, who they are, and how your ideas will fit together always made sense to me.

Next, Julienne used the analogy of "feeding off" other colleagues for professional resilience and subsistence, which initially inspired my use of foraging as a metaphor for connectedness and for participants integrating with their professional surroundings. She shared,

The relationship piece is critical, and you must seek it out. Working in this way is hard, but that is how I came to the work. It is important to have other people like you, who are also wanting to be Reggio inspired, are going to Reggio Emilia Italy and to seek out these educators who are inspired in their practice to feed off.

Joni picked up from there,

I was thinking along more of a straight line, like history. By college I was learning about Piaget as a constructivist and Vygotsky as a socio-cultural perspective. My experience has always been in schools that had a social constructivist approach and that valued community so even if we weren't saying "we are social constructivist" we were. 
Joni expressed a pivotal experience early on in her career when she realized that the Reggio Emilia educational system was aligned with the anti-bias values she identified with,

My thirty years at the [laboratory $\mathrm{CDC}]$ center and a strong commitment to social justice shapes how I think about the world and has given me an openness to perspectives. Early on my immersion in the anti-bias community kept me from going to the Wonder of Learning - it wasn't called the Wonder of Learning then-

At this point Julienne interjected the question, "You mean The Hundred Languages?" Joni confirmed that she was in fact referring to the museum exhibit: Yes, the Hundred Languages of Children exhibit was here in town. I don't know why I didn’t go, it wasn't an active choice. Reggio inspiration wasn't on my radar, or in the circles that I was in because my professional connections were all in diversity and equity work, so I was not an early adopter! [Laughing]. When I went to the first conference workshop about Reggio Emilia schools and Reggio inspired work, it clarified for me that Reggio inspired values were aligned with anti-bias work.

Joni communicated an optimal foraging strategy as the ability to select and combine pedagogies and identities that supported her growth. For Joni to digest the pedagogy, it was essential that the Reggio Emilia approach integrate rather than conflict with the anti-bias values that had transformed her consciousness. Joni shared, 
Conversely, when we actively started exploring the anti-bias work there was a lot that I had to let go of from my teaching past. It was incredibly exciting, a whole revolution that I was happy to be a part of, but it felt like I was going one way and now I've got to go another way. When I learned about Reggio Emilia philosophy I thought, wow! That is the way you articulate what we are trying to do-new ways that we can do this better-do this work powerfully. I was relieved it didn't feel like I must stop something that I am doing, change what my other work is, and shift it into something else.

Over time, as I reflected on the interview data, I realized that Joni brought a unique layer to the narrative project. I began to interpret her central interview idea of "Reggio Emilia is an equity-based pedagogy" as substantiating an image of the child as a competent global ecological citizen, capable of understanding and expressing more complex features of social identities.

Now, let's turn to the Remida-inspired classroom teacher dyad that took place about two months later.

Classroom Teacher Dyad. The third dyadic interview in narrative session one was with a stranger-based pair of classroom teachers who met on a Saturday in April-Clara from the laboratory $\mathrm{CDC}$ and Miranda from the corporate CDC. In response to the ice-breaker prompt - share what it was like to represent yourself with reuse materials — both classroom teachers described experiences of broadening the scope from physical to relational: 
Miranda: It's always a bit awkward for me. I feel more comfortable thinking about someone else, especially children. But it is good to slow down and think about what is important to me; how I view myself. I was trying to represent myself honestly which in a physical way is plain and natural, so I used leather and rocks and rusty circles. After I was finished with the face part, I felt there was a missing piece. I wanted to represent that I have a busy brain, so I used gears because I am usually thinking a lot, trying to solve problems; it takes some work for me to slow down my brain.

Clara: I have been exposed to this work many times. For the first time I was focusing on how do I see myself? How can I truly represent myself with these materials? I was excited when I saw this chain was perfect for my hair! [Laughing]. These little rocks were perfect for my eyes; I am very connected to nature, so I didn't want bottle caps. I felt more connected with the work this time because it was a true representation of myself. I focused on what I wanted to use instead of what will happen if I use that, you know?

Miranda reflected to Clara, "I like the idea of what material spoke to you." Miranda and Clara's reflections during the hands-on collage portion of their dyadic interview relates to social identity that came directly out of their experience of working with the reuse materials. 


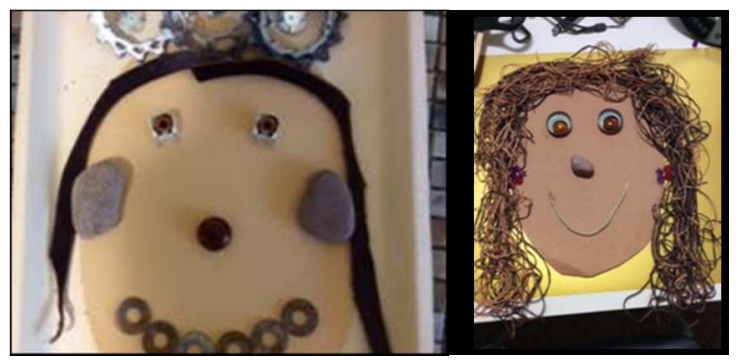

Figure 4. Clara's (left) and Miranda's (right) reuse self-representations.

After making self-representations out of reuse materials (see Figure 4), the pair of classroom teachers narrated how they came to identify as social constructivist and Reggio- inspired. Miranda traced back how the first college she started out at had a strong early childhood program. The course work was social constructivist, and her first placement for student teaching was in a college-based Reggio inspired lab school. The woman leading the classroom said Miranda, was in her sixties and had just returned from a study tour of Reggio Emilia. She then specified that it was in early 2000 when North American teachers were just starting to travel to Reggio Emilia, Italy.

As the youngest members of the participant group, the classroom teacher dyad shared generation-related experiences that included being enculturated into Reggio inspired early childhood education early on in their professional formation. Both Miranda and Clara began their practice in Reggio inspired learning communities during the last 10 years. Like studio teacher Maggie, Miranda also used the analogy "dig into" to describe her experience of foraging for pedagogical roots to give endurance to her practice, 
I had a student teaching experience in a more conventional public-school setting, and I also student taught in a kindergarten, so I had diverse experiences that I could dig into that helped me to figure out what made sense to me.

Clara outlined how she first started working in the [early] childhood field 10 years ago in San Diego in a Reggio-inspired school. That is when she fell in love with the Reggio philosophy. She went back home to Brazil and then came back and got her master's in Oregon,

That is how the whole idea of social constructivist practice came together; what does it mean to be a social constructivist? Along with the idea of the Reggio philosophy, it means giving children the opportunity to learn by interacting with one another and playing together, exploring the materials and exploring their ideas. And to share their ideas with one another alongside negotiated curriculum with the teachers.

In Clara's concluding narration of how she came to identify as a social constructivist, she used the metaphor of democratic education as healing, to describe her experience of Reggio inspired education,

That is the beauty of the whole philosophy; it heals and changes people's lives. If you grow up in an environment like that, you are becoming self-confident; trusting yourself and exploring your ideas, speaking your truth because you know you have the freedom and the right to speak your mind.

Over time, as I reflected on the interview data, I realized that Clara brought a unique layer to the narrative project. I began to interpret her central interview idea of "The 
Reggio Emilia approach heals through democracy" as substantiating an image of the child as a competent global ecological citizen, capable of understanding and expressing more complex features of social identities.

Now that I have presented key narratives of nourishment and resilience the participants have experienced as Reggio inspired educators, I will turn to the professional formation patterns that came into view. I conceptualize the data using Katz's (1972) survival stage framework. Further, I align characteristics of becoming a Reggio inspired educator as part of a Remida creative reuse materials adoption cycle, with the corresponding forager archetype that emerged.

\section{Data interpretations one: Survival stage growth patterns-The Forager.}

Through a close examination of the data, four patterns came into focus accompanying the archetype of the Reggio-inspired educator as Forager: (a) Gathering pedagogies; (b) Relationality as an adaptive strategy; (c) In search of theoretical frameworks; and (d) Sustaining situated learning. In the following discussion, I will describe these patterns in greater detail. To highlight collective patterns, I focus on participant responses to prompts from various data sets.

I interpreted the first data pattern after the interviews were shared through the forager lens: Gathering pedagogies. Here, I connect the collective experiences of participants as they struggled to make sense of the pedagogical hunger they encountered in their early professional lives to characteristics of the first stage of survival as teachers question their professional competence and their desire to teach. In the survival mode, teachers are focused on themselves and their own needs and have less understanding of 
their student's needs (Stroot et al., 1998). According to Katz (1972) this preoccupation with survival may be expressed as, "Can I really do this kind of work day after day?"

To explore how social identity and Remida materials are connected, participants worked in pairs to make impermanent and permanent collage representations of themselves from a wide display of reuse materials. Our inquiry was guided by the enduring question: "How can we connect ourselves with reuse materials and see ourselves through reuse materials?" Inspired by the provocation, participants engaged in a reflective dialogue process.

Studio teacher Maggie's expression, “That is how we as educators, and how I personally, identify things about my practice — by starting from what I know I don't want" summed up the participants' experiences as they struggled to make sense of the pedagogical dissonance they encountered in their early professional formation.

Although teachers in the survival stage of their career may have a lesson that they have planned, it is common to see them go through the lesson as if it was a script that cannot be changed. There is little recognition of the children's reaction to the lesson and they typically make few modifications to the materials or plan to address the divergent needs of the individual children or small groups in the classroom (Stroot et al.,1998). Maggie recalled feeling frustrated by following a teacher-directed map that was all laid out and at the same time she remembered the initial anxiety associated with sampling an emergent approach.

Classroom teacher Miranda used the analogy of disequilibrium to describe her discomfort with the imbalance of teacher-directed curriculum and how she discovers 
balance through a Reggio-inspired approach, "It all lined up, my previous experiences of feeling disequilibrium and then interviewing at both Reggio inspired centers. This will be my ninth year at my school. I feel so lucky; I love it!"

Miranda also shared her experience of gathering diverse pedagogical experiences that she could dig into during her first year. Like the other participants, she had a common experience of cognitive dissonance early on in her professional formation that led to developing a strong social identity as a Reggio-inspired educator. Miranda recounted,

When there is 'preschool' put on the front of the word teacher, the connotation in our society and the value is so low. We are co-researching alongside children. We have studied child development and learning. I identify as a preschool teacher, but at the same time we are teaching each other, we are in this experience together and every day is a new moment to co-research together (Miranda, 2016). Each participant articulated the instinctual experience of deducing what sustained them professionally by moving away from pedagogies that deprived them of strength and vigor. This growth pattern resonates with Pinkola-Estes' (1992) motif of the huntress used to represent the psychological elements of humans that seek to know, that strive to nourish the self through merging with their instinctual nature.

The participants could easily recollect at least one time when the experience of pedagogical dissonance inspired them to draw upon their instincts to adapt the curricula to the social identity of the children they were working with at the time. Maggie shared 
an example of how as an educator she identified intentional aspects of her practice by starting from what she intuitively knew she did not want to do:

I left the first center I worked at after I had my first child and the epiphany of oh, I don't want him to go here, even though it was a great school and the people had good intentions. I realized something was not resonating with me since I didn't want my only child to go to school there. I started ticking off what I didn't want, for example: I don't want children to sit at tables and desks, or to have assigned seats on the carpet, or to do worksheets.

Each participant shared that they engaged in a wide search to identify with a pedagogy that resonated with them intuitively. Likewise, Maggie noted to her dyadic pair,

We went to Hilltop [Institute located in Seattle] and to the first ever [Opal School] Reggio inspired symposium where we met Judy Graves who was a huge inspiration for me, like you were saying Christine and Lucy Chaillé were for you. Everything started coming together and I think I would identify that last ten years as the biggest growth too.

Similarly, Joni pointed out, "When I went to the first conference workshop about Reggio Emilia schools and Reggio-inspired work, it clarified for me that Reggio inspired values were aligned with anti-bias work."

Drawing on foraging and gathering metaphors, each participant instinctively knew what fed their practice before they could name it and continued to hunt for pedagogical strategies until they found ideas that fit with their own developing 
pedagogical perspective. In order to relate to these perspectives, the participants' experiences seem to move to a form of relationality as an adaptive strategy for professional survival in their stories.

A second data pattern emerged through the forager lens of relationality as an adaptive strategy once I could see the first pattern that showed each participant's sharing of pedagogical perspectives. Here, I connect the collective experience of discovering resilience through a learning community to characteristics of the first stage of survival for teachers; teachers whose main preoccupation with survival may be expressed with questions such as, "Will I be accepted by my colleagues?” During this survival period, the educator is most likely to need support and guidance through relationships with likeminded colleagues (Katz, 1972) as she grows in her pedagogical perspectives.

Julienne's observation that "it is important to have other people like you, who are also wanting to be Reggio inspired," summed up the attitudes of participants as they struggled to make sense of their hunger for collaboration they encountered in their early professional lives. Ann used the metaphor of feeling like her "head was going to blow up" to describe her cognitive experience of joining a culture of teacher-researchers.

One of the things that shocked me when I transitioned to [the lab preschool] was the group dynamic between the teachers and the director. When talking about our curriculum where I worked before, I was like, hey, I have an idea! And I remember someone saying, when don't you have an idea? [Laughter]. And I said, it is boring to do community helpers every January when you have been doing this for a long time. Let's think about doing some other ideas that the children are 
interested in. I wasn't at the point where I was asking the children to tell me what they were interested in because I wasn't reflective yet.

Ann continued to share about the intellectual dimensions of learning alongside Reggio inspired educators at the laboratory CDC,

When I got to [the laboratory CDC] center, everyone had a good idea! [Laughing] Teachers were including what the children said about curriculum. I remember going home and thinking I am in an entire school full of people who don't want to do the same thing all the time! That was my first beginnings, I can remember feeling like my head was going to blow up, [Laughing] there are so many good ideas!

In the modern-day forager context of the participants' professional formation, their primary resource for resilience was relationality or building community through collaboration. Referring to children, Clara says: "And to share their ideas with one another alongside negotiated curriculum with the teachers." Clara also says: [Democratic education] "heals and changes people's lives. If you grow up in an environment like that, you are becoming self-confident." Joni says: "My experience has always been in schools that had a social constructivist approach and that valued community." All of this relates to being in a community. A community is necessary for growth for young teachers. It also helps them reconceptualize their pedagogy. In fact, the importance of relationality surfaced in the data as being at the heart of Remida-inspired participant's experiences of identity studies. 
The stories the participants told about how they became Reggio-inspired shared one common theme: professional nourishment though their relationships with micro and mesosystems including Reggio inspired early childhood university programs, colleagues, supervisors, literature, professors, conferences, and their respective schools.

Relationality is key during the survival stage as the educator needs direct help with specific skills and insight into the complexities of children's individual and social identity expression. On-site mentors may be master level educators, advisors, consultants, and directors (Katz, 1972).

Further, the narratives that emerged from partnered reuse self-portraits were all relational stories, and in the process of representing themselves with reuse materials, each participant continually referred to important people in their lives-spouses, children, coworkers, students, extended family and each other. This pattern highlighted the social construction of their individual identities through both contrasting and comparing themselves to other people. The pattern illustrated how conceptualizing identities as a source of data can reorient theories about the relationship between the individual and the social by calling attention to the cultural dynamics through which individuals construct themselves as social actors (Sfard \& Prusak, 2005).

This reuse self-portrait provocation was designed to make visible social identity formation in a Remida-inspired way. Creative reuse paired with reflective discussion assisted the participants in articulating their experiences of becoming Reggio inspired as the next level of formation as a professional. During the provocation, the participants often considered their relationships with the children they work within their self- 
representations. For example, studio teacher Maggie noted, "I was thinking about how children are always obsessed with nostrils when they do these self-portraits, and so I was trying to find the right nostrils." To this Ann replied,

Me too! [Laughing] Children seem to fixate on their nostrils especially when they look in the mirror. I chose this seashell for the same reason, because it looked like a button nose and had two holes. I don't think as an adult I would have necessarily focused on that, but I see that the children do that a lot, and I wanted to represent that.

Another example can be found when participants reflected on authenticity and identity through their creative reuse. For instance, Clara remarked, "I put a smile on my portrait because I am happy doing this, and to be in this field. A smile is a big part of my personality, it is real to me and authentic." Miranda responded to this comment by saying:

The last time I did this experience with a group of educators, we represented the person next to us and for me, it was my co-worker. I liked doing that because I could share some of her gifts and strengths with her through my representation of her. In myself it was a little bit harder to bring those forth.

This dyadic narrative data documents how hands-on work with reuse materials tends to elicit reflections and representations of social identities and relationships. This notion of participants narratives of searching for theoretical frameworks is shared below.

The third forager data pattern to emerge was: In search of theoretical frameworks. Interactions with local Reggio-inspired advisors, consultants, and directors contributed to 
the development of a theoretical foundation that supported the participants' practice and formation of their self-concept as co-researchers with children, materials, and environments. From these narratives on interactions, I began to connect the collective experiences of participants' search for theoretical frameworks to characteristics of the survival stage (Katz, 1972), wherein the novice educator is most likely to need insight into the complexities of children's individual and social identity expression.

The participants' collective experience of struggling to express the social constructivist approaches was summed up in studio teacher Maggie's statement, "This is what we are doing, what we don't have words for, that we can't explain." She articulated how the combination of the environment and Reggio inspired literature left deep impressions on her,

I started working at the [corporate $\mathrm{CDC}$ ] and was intrigued by the environment; it is so cozy with couches and the children were playing. In 2001, my co-teacher and I read a Reggio inspired book by Louise Caldwell and thought to ourselves, this is what we are doing, what we don't have words for, that we can't explain. During the dyad discussion, studio teacher Ann related that she does not identify as an 'atelierista' and shared a story to explain why, which helped her develop her 'offness' theory. Ann reexamines this moment closely,

When Vea Vecchi came here and walked through both my and Marsha's studios, she asked Will [Parnell EdD]_-'Are they teachers or artists?' To which Will replied that I had a great background knowing the different kinds of materials and how to use them with children. And Vea says, 'So, she is not an artist?' and I 
thought, well, I do a lot of art at home and have sold it at Saturday market (laughing)!

Maggie also related, I have never identified myself as an atelierista for the same reason that I would never say that I work for a Reggio school. I am not from Italy, that is not my context or reality and never for even a second has been. I am inspired, and they inform my practice, but I am also informed by RIE, by Montessori, and by my own experiences with art as a parent and a teacher.

Instead, both teacher participants shared that the title studio teacher makes sense to them because that is a hundred percent their context. For example, Ann shared that her job involves showing children artistic techniques as she shared, "This is liquid water paint, this is the tube, you make a little dot, and this is how you use it." She went on to say,

I believe strongly in Vygotsky's work scaffolding for children and that's what I bring to the program, and to that part of their school day. I know children, and Reggio schools' children can do amazing things but sometimes what I see in Reggio Emilia photos or in the old movies like The Amusement Parkfor Birds looks like a grown up with a very strong art background has said, "No, this is how it should go."

Maggie responded strongly to this description saying "Yeah!" to indicate that this description of her role made sense to her. Ann continued, 
I could be wrong; I haven't seen the Italian children represent things by themselves. For example, when children at [the laboratory school] are making a face with clay and it is not what I think they are capable of, I might ask them, 'I notice you have one big eyeball and one little eyeball, is that what your eyeballs look like to you? Is that how you like it?' and if they say 'Yeah, fine.' I am like 'Cool, I don't care' because that is constructivist, that is our style. I wonder if they do that in Reggio Emilia or if they say no, stop! Really go look at yourself! I wonder because I don't see their work having as much 'offness' as our work does, and I don't believe that our children are less capable.

Maggie responded to Ann's observation by relaying, It is interesting that you came full circle, from feeling judged by Vea Vecchi for suggesting that you are not an artist and can't do this job versus what you just said about how we don't know what is authentic because we don't understand each other's contexts. Hopefully we can exchange some inspiration with the Italians and everybody can be a little better for it in the end.

When asked how they identify professionally, all of the participants rejected imposed titles, and each articulated their own social construction. Studio teacher Ann revealed how dominant constructions of ECE identities that the participants considered as originating from society at large or from the municipal schools of Reggio Emilia were contested in creative ways, inviting the co-construction of a multiplicity of Borderland identities. 
Throughout the first session, participants chronicled their careers and memories into a series of narratives, marked by theoretical frameworks that shaped their becoming Reggio-inspired. Interactions with Reggio inspired advisors, consultants, and directors contributed to the development of Ann's critical theory that supported her practice and formation of her self-concept as an educator. To further illustrate the forager metaphor in classroom teacher Clara's story, she garnered the theory behind social constructivism and the Reggio Emilia approach. She fell in love with and optimized her survival as a neophyte and the professional fitness that comes with that survival over her past years.

This search for theoretical frameworks illuminated the path to a fourth data pattern through the forager lens as sustaining situated learning. It is interesting to note that the outstanding practices to be observed in the small Italian city of Reggio Emilia that are admired worldwide are implemented by teachers with only a high school education, but with extensive and intensive on-site professional formation supports (Edwards et al., 1998). In this study, narratives about how graduate degrees in ECE combined with hands-on experience helped participants to theorize what made common sense to them, aligning them with characteristics of the survival period. For instance, studio teacher Ann described her increasingly hands-on experience in the classroom and studio as "the combination of academia and practical" to explain how she came to be Reggio inspired. Anne said, "I started the masters [degree] program and began to understand the theoretical aspects of what social constructivism meant day-to-day with children; the combination of academia and practical." Ann's statement made me 
consider how Lave (1996) argued that learning is situated and embedded within the activity, context, and culture.

Feedback from participants in this study indicates that educators in the emerging period of their career benefit greatly from in-situ facilitation to help them to interpret how to apply educational theory to their practice. As Ann noted, "Just trying to figure out all the theory and how to practice it is a huge challenge. Most educators who have worked in any other school can understand the concepts, but the daily practice is very different." This illustrates the importance of situated learning theory, which, is also congruent with constructivist theories of learning as a social advancement that involves language, realworld situations, and interaction and collaboration among learners.

The combination of academia and practice also summed up a common observation among participants regarding how their graduate degrees in ECE combined with hands-on experience helped them theorize what made common sense to them. Ann used the analogy of "balance" to describe her experience of moving out of the disequilibrium of teacher-directed and thematic based curriculum towards a Reggio inspired co-learner approach that she found more professionally fulfilling. Her experiences of being sustained by mentorship were part of a larger pattern of collective experiences echoed by many of the participants during situated learning. Ann illustrates, I have had wonderful mentors, read Reggio inspired books, I have been doing the work and seeing the shift from teacher directed, thematic work to a balance of child and teacher together as co-learners. I was always at the edge of that 
approach, kind of on the top, and now I see it more as that balance between the two.

The pattern among participant's narratives — struggling to find language to express the constructivist approaches that came intuitively to them-re-surfaced in classroom teacher Miranda's narrative. Like other participants, Miranda noted theorists who impressed her during her graduate studies in ECE and shared that the theory made sense to her. She explained, "From the beginning, my education was social constructivist, so I was learning about Dewey and Bronfenbrenner theories, and it just made sense seeing it in practice." Miranda's experience echoes Lave's (1996) theory that learning is situated and embedded within activity, context and culture.

During Katz's (1972) Stage I, the neophyte acquires a baseline of information about what young children of a given age are like and what to expect of them. Miranda also used the metaphor of an explorer to describe the image and identity of the child that began forming for her early on in her practice when she said, "My lead teacher had just gotten back from Reggio Emilia Italy, so I heard a little bit from her and watched and explored this idea of children as researcher and as explorer." This statement reminds me of Lave's (1996) theoretical work in situated knowledge and learning, which is aligned with a Chicana/o studies, social constructivist and Freirean image of the learner as a cocreator of knowledge.

Also, classroom teacher Clara echoed the participants' collective experience of struggling to find a theoretical language to express the social constructivist practice- $-\mathrm{a}$ practice that came instinctively to them. She said, "Reggio inspired work felt so right 
because it is all about the children and the respect we have for them as individuals, for their ideas and their desire to learn." Clara speaks to constructivism as a theory of learning rather than as a method of instruction. The pattern of situated learning emerging from the data highlights the importance of the relationship between learning and the context in which it takes place. The dyadic interviews provided time and space for participants to narrate how they came to identify as Reggio-inspired and contribute something unique to our understanding of how these educators transform Remida theory into practice and vice-versa.

Now that I have presented the data and interpretations, I wish to represent the main ideas that the participants shared using a methodology known as re-storying. The following re-story combines six of the participants' voices into one protagonist who shares the experience of struggling to find a sense of professional belonging. The main character's struggle comes to resolution through socially identifying as Reggio Emilia inspired and social constructivist in her approach. Participants' metaphors are italicized in the re-story, which is also presented in single spaced to privilege its narrative quality. This was done to highlight their significance in my multi-metaphorical (Sfard, 1998) interpretation of the data.

\section{A re-story: The Forager.}

My student teaching experience was in a more conventional setting. In that school, the teachers had the map all laid out; the curriculum was the same yearafter-year. Then I started working in a Reggio-inspired center with a lead teacher who had just gotten back from Reggio Emilia Italy. This co-teacher envisioned designing learning experiences for children that placed them in the role of researchers or explorers. I initially thought that developing social constructivist curricula was going to be hard work, but I gained diverse experiences that I could 
dig into and figure out what made sense to me. In the beginning, I can remember feeling like my head was going to blow up because there were so many good ideas in my Reggio-inspired learning community!

My professional formation involved my previous experiences of disequilibrium and then discovering Reggio inspired early childhood university programs, colleagues, supervisors, literature, professors, and conferences where I found a sense of belonging. When I went to the first conference workshop about Reggio Emilia schools and Reggio inspired work, it clarified for me that Reggio inspired values were aligned with anti-bias work. That is when I fell in love with the philosophy, with the Reggio philosophy because the Reggio Emilia Approach is equity-based pedagogy, and a democratic approach is healing. Together, my coteacher and I went to our director, holding up a Reggio inspired book saying we wanted to learn more about the Reggio approach and how to practice it. Our director suggested we dig in. It is so important to be connected to other educators who want to be Reggio inspired, are going to Reggio Emilia Italy, and to seek out other educators who are inspired in their practice to feed off because working in this way is hard, but that is how I came to this work and what sustains my professional identity.

The last 10 years of teaching have been my strongest journey into what Reggio inspired work looks like because previously I had only experienced the bubbling of what constructivist work could be. I have been doing the work and seeing the shift from teacher directed, thematic work to a balance of child and teacher together as co-learners. I have struggled with always being at the edge of that approach but now I see it as a balance between the two. Part of that is the growing community, there are so many more people to talk to now and share ideas and collaborate with that it just blossoms.

\section{The Forager summary.}

Participants used the metaphors map all laid out; explorers; dig into; dig in;

feeding off; strongest journey; bubbling; being at the edge; and blossoms to describe their experiences of becoming Reggio-inspired educators, which led me to interpret their early professional formation through a forager archetype. The modern-day forager as a metaphor corresponds with Katz "survival stage" or first year of teaching where the overriding concern is daily challenges and the primary resources needed are local pedagogical support. 
For millennia, humans were hunter-gatherers, and some suggest we have a prehistory of cooperation and foraging recorded in our genes. The liveliness of using our senses to find nourishment as opposed to the passivity of consumption is central to both our biology and our interpersonal growth. Reflecting on the narrative data led me to see patterns of professional formation in becoming Reggio inspired that led to the adoption of Remida pedagogy.

Now that I looked at the first stage of formation of Remida-inspired practice through the metaphorical archetype of the forager, I turn to the narrative data collected, interpreting them through the metaphorical archetype of the global homesteader, which I align with Katz's (1972) consolidation and renewal stages.

\section{The second narrative inquiry prompt: The Global Homesteader.}

The second prompt was intended to elicit responses to better understand participants' experiences through exploring their professional formation in the Remida approach. This prompt, "Please share the story of how you came to be Remida-inspired," was designed to better understand participants' experiences of adopting creative reuse into their social constructivist practice. The intention in the first session of dyadic interviews was to create an opportunity for participants to deeply consider their thoughts and feelings about their experiences with creative reuse cultural education, and to begin to articulate their underlying belief structures about working with reuse materials in Reggio-inspired identity studies.

Participants used the following metaphors to describe their experiences of becoming Remida-inspired educators: spark; coming home; dug into; harness; 
groundwork; footprints; captured; beef up; transformation; flows through the veins; hitting a juncture; little bump; next layer; fine tuning; and dream. Although each participant's experiences were unique, patterns of becoming Remida-inspired came into view which I interpreted through the archetype of the global homesteader; this symbolic image is not to be mistaken with settler occupation or colonialism.

Use of the term "homesteader" in North America dates to the Homestead Act (1862) and before, however the archetype is not to be mistaken with the concept of manifest destiny. This clarification is a profound distinction. Take for example the historical and spatial dynamics of the geographical border between the United States and Mexico that has been largely shaped by the U.S expansionist agenda. The doctrine of manifest destiny in this case resulted in Mexico losing half of its territory and the subsequent marginalization of Chicana/o's who were foreign others because they did not share the same values, culture, religion, traditions, and skin color as the new majority population (Orozco-Mendoza, 2008). Hence, a colonial interpretation of a Homesteader metaphor would be antithetical to the Borderlands conceptual framework, a key theoretical concept that underlies this study.

In this instance, the metaphor is symbolic of working towards a life where homesteaders around the world grow early childhood education for sustainability, creatively reuse materials and research Remida-inspired identity studies in a way that "fosters intelligent moderation in the next generation" (Parnell et al., 2017) with a local/global ecological consciousness. 
I use the archetype of a homesteader to further organize and interpret the findings. As mentioned above, the Homesteader archetype corresponds with the consolidation and renewal stages. It is based on the premise that the professional growth of early childhood educators generally occurs in stages associated with survival, consolidation, renewal, and maturity (Katz, 1972). In this instance, the term includes the word "global" as a symbol of working towards a life where homesteaders around the world grow early childhood education for sustainability, creatively reuse materials, and research Remida-inspired identity studies in a way that "fosters intelligent moderation in the next generation" (Parnell et al., 2017) with a local/global mindset.

In these second and third stages of professional growth, the educator's overriding concerns are problem situations and professional renewal. More specifically, the early childhood educator who is in the consolidation stage has usually come to see themselves as capable of survival, is ready to consolidate the progress they have made, and to differentiate skills and tasks to be mastered next. During the renewal stage III (typically in the third or fourth year of teaching), educators begin to ask questions about new developments in the field such as who is doing what and where; and what new materials, techniques, approaches and ideas, are emerging.

As with the survival stage, the primary resources needed at these stages are access to specialists and collegial advice as well as to local, national and global conferences, professional associations, journals, and visits to demonstration projects. However, the way in which educators draw on these types of resources at this stage is different than in the initial survival stage. 
For example, the participants in this study described using the Remida approach as being a source of renewal and refreshment in the second and third stages of their professional formation. Recall that the Remida approach represents a new way of approaching environmentalism and building change through giving value to reuse materials in ways meant to intentionally provoke new opportunities for communication and creativity based on the perspective of having respect for materials, nature, and human beings.

As I focus in on studio teacher Ann and Maggie's session together, I find new metaphors in their stories that yields data that are both unique-to-the-individual and universal-for-the-individual symbolized here as the archetypal global homesteader.

Studio teacher dyad. Each dyadic pair responded to the second narrative inquiry prompt: "Please share the story of how you came to be Remida-inspired?" First is Ann and Maggie's time together.

Ann: When I first came to [the laboratory CDC] the library was a reuse area. That was the first time I had that many discard materials available and creatively reused materials to that degree. And, I also suppose I came to be Remida-inspired through having the Inventing Remida Project develop upstairs.

Maggie: I experienced a real frustration with the way resources were used; it was building to a high level for me prior to my first trip to Italy. When I was there, I was reading about the Remida center in the schedule of events and I thought to myself "Yes, this is what I want to know!" 
Maggie used the same metaphor to "dig in" that she used to describe her experience of becoming Reggio inspired to describe her colleagues' inspiration to travel to the Remida Recycling Center in Italy to learn more about the roots of the approach. Maggie continued, "The group of educators that started creative reuse work went to Italy to work with the Remida center and dug in. That helped me to identify as being Remidainspired before going to Italy." Interestingly, the phenomena of becoming Remida inspired before visiting Reggio Emilia Italy was an experience shared by Maggie (and classroom teacher Miranda) from the corporate CDC.

Further, Maggie and Ann's experiences of working in a Remida-inspired way around identity studies included issues of impermanence. Maggie had become increasingly intent as a Remida inspired studio teacher on not fastening materials together permanently and clarified, "It is important to me now that we not glue and staple everything together. It is important not to alter material in such a way that it can't be used again." Ann paused a moment and located herself more in the middle of a spectrum of impermanence, "I go in-between those two, sometimes we glue things and sometimes we use loose parts.” Maggie agreed, “As a teacher, learning things like if you really want to attach materials, how to do it so that they can still come apart with a minimum of waste is important." Ann confessed, "I still glue!" and the pair laughed heartily, she shrugged and continued, "But I rip them apart. The few things that I can't rip apart I just think, Oh well! That is the place I have come to." 
Maggie then shared her first personal encounter with Remida in Reggio Emilia, Italy by using the metaphor of coming home to describe her experience of the environment as the third teacher,

I had an intense feeling of familiarity, of coming home, of this makes sense to me, when I walked into their second room which at the time was all light - it had very high ceilings and all the overhead lights were off. It was filled from floor to ceiling with projectors, there were discards [art pieces made with reuse materials] hanging ten feet high all the way down to the floor, and light tables with tiny little pellets spread out across them. Reuse materials were projecting, reflecting and refracting all over the walls.

During Katz's (1972) professional growth stage II consolidation, an educator is likely to differentiate specific tasks and skills to be mastered. According to Katz, specialists can strengthen the teacher's skills and knowledge at this time. Exchanges of information and ideas with more experienced colleagues may help an educator master the tasks of this stage.

Maggie then referred to SCRAP, a non-profit creative reuse cultural center located in the Pacific Northwest that was instrumental in installing the creative reuse cultural center's in both CDC's involved in this study. Maggie used the metaphor "flows through the veins of our school" to describe how the social identity of the corporate CDC became Remida-inspired,

We ended up working with SCRAP in [a]... capstone class one year, who helped us put a reuse center in our school, and then it grew and grew for me. At first it 
was awkward because Remida-inspired education is hard to understand and to put into daily practice. People try to make creative reuse this big separate project compared to now years later, it's in the veins of our school - it flows through the veins.

Maggie's organismic metaphor supports scholarship that states the Remida creative recycling center holds a vital place in the "living system" (Cadwell et al., 2005, p. 115) of Reggio Emilia, Italy and increasingly so in global Reggio-inspired educational systems. Maggie, like Tarr (2003), described the many natural and recycled manufactured Remida materials in Reggio Emilia classrooms and how the use of materials is deeply embedded in and integral to the life and culture of the classrooms. The term "affordance" was used to describe how each medium including complex reuse materials, provokes a special orientation to the problem to be solved, thus assuming a critical role in the concept of the Remida-inspired conceptualization of the environment as the third teacher.

Maggie went on to use the metaphor of "fine tuning" to describe her learning community's experiences of consolidation and renewal through working in a Remidainspired way,

It was a huge learning curve, but we got to the point where it is just natural for us and part of who we are. Now we are fine-tuning the education piece and what that means for teaching children about the environment through creativity and play versus through fear- "We are destroying the planet"-working naturally 
with the way they live without trying to terrify them. Not that you ever arrive, but we are on the higher level of our processes.

Now that I have presented studio teacher layers of experiences of becoming Remida-inspired within a global context, I turn to the narrative session that followed two months later with the classroom director dyad. I begin with Julienne reflexively considering her own experiences and impressions.

Director dyad. Director Julienne used the metaphors of "harness" and "groundwork" and "footprints" to describe her experience of building community through local and global ecological collaborations to plant and tend to a Remida-inspired center at the corporate CDC:

Julienne: We wanted to harness collective resources and not to do it by ourselves. We got started by talking with SCRAP who shared the same mindset, then our footprints or our ideas were shaped by [University] undergraduate students who had to do a reuse project [Laughing]. That is how we got started, the capstone class did some groundwork for us. That is how we collectively began. Joni: It used to be that our library was our reuse space and I think there is something great about having that publicly stated value, and an invitation for families.

Julienne: Yes, and you can also see where your values are crumbling! [Laughter] It is a good thing to notice because it is in such a public place. You notice right away if it gets disorganized, misused or not tended to because it is in the entrance to our school. 
Joni: You can't not see that's happening!

Julienne: It can become an eyesore. I think, Oh, this doesn't make sense anymore — or, we are not giving it enough time or attention — therefore do we really value this, and are we bringing this forward? Or is this just nice stuff? It's a conversation we have a lot [Laughing] as a way of understanding whether we are working towards connection or are we disconnected? [Laughing] If that makes any sense? It's a lot of material, it is visible, and it tells you a lot. Joni: It can tell you—oh my gosh, we are stretched thin right now! Julienne: Or, we are out of control with all this material! [Laughing] Both creative reuse cultural center's share similar challenges in stewarding the space by collecting, organizing and displaying reclaimed materials. Julienne used the metaphor of "beef up" to describe her experience of being inspired by Remida to harvest a reuse materials philosophy,

Remida discards support ideas or projects in the classrooms; we might beef up one collection or type of material that has attributes or affordances to support the work. People look at the reuse materials in different ways for different things and sometimes the whole school is exploring a big idea. This year the intention is creativity which brings out a different thought process and shifts the way we want [the creative reuse cultural center] to look. Then there is foundational work that you always do, such as developmental work where like colors are grouped together so that if children have an idea they know there are lots of purple things in [the creative reuse cultural center]. 
Then, Julienne used the metaphor "captured," to describe her experience of display and pedagogy,

We had already captured the piece about aesthetics and wonder, and what the materials might tell you. A big part of working with the materials after that was to get them out of the closets, accessible to the community and to see what this kind of commitment or value [they] would bring to our program. It is still a lot to organize, manage and source. Our reuse center is currently located in a central part of our school and has become a place of connection like the fish tank or the front office.

Next, the dyadic pair responded to the second narrative inquiry prompt: How did visiting Reggio Emilia Remida impress you?

Joni noted that their study tour was in 2003 and wondered, “Actually, I didn't go to Remida, I haven’t been to Remida. Did we actually go to Remida?” Julienne remembered that the pair had engaged in a reuse materials provocation during their study tour. They both recalled how they were given gifts such as fans made with recycled materials. Joni further pondered, "Maybe it [Remida] was much less developed at that time?" Julienne responded, "I guess you are right, the Remida as it is now, we haven't actually been to but-remember all the encounters we went on, like that little tour?" Julienne then continued to reflect more at length on her first impressions of Remida pedagogy;

When I saw how Remida was using the materials, the impression I had was of course! Why don't we look at materials that way? Our program was still buying 
things that could have been reclaimed and reused. Remida told stories about how they built relationships with people who were contributors of discards. I wondered if I had the capacity to forge those relationships so that the materials would be rich, or even know how to ask for something that looks like the materials. Early childhood teachers often wonder how to reuse materials for conservation of financial resources but Remida was a different mindset - the mindset of sourcing it so that there would be a community partnership.

Joni in turn reflected more deeply, Early childhood people have been great, historically at repurposing and reuse. What was so different about Remida or the way Remida materials are used in Reggio Emilia schools is how much open-ended repurposed material was available to children. The ratio of reuse materials to those that had more of a close- ended use manufactured for children. Some of the Reggio Emilia schools had a huge proportion of Remida materials instead of legos and baby dolls, especially the infant toddler centers-I was really struck by that.

When prompted by this researcher to elaborate on how as directors, they translated their impressions of Remida into practice upon returning to the Pacific Northwest, Julienne shared, We source materials from parents in the beginning of the school year and sometimes in the middle. During a big project, we will ask families for certain things like extra shiny discards: Can you save them? Can you look? Do you 
know anyone who can source them for us? Reuse materials are collected from homes and circles of relationships; families will say, I have a brother who is an architect, so he has these bendable pieces, do you want them? We are trying to forge those connections. It is hard work to make that important to busy people; all of us are very busy people. But the children help us because they like the treasures [Laughing]! They see it as treasures rather than junk, or through other lenses.

Julienne described how the corporate CDC has a creative reuse cultural center in a central part of the school and has become a place of connection. Joni reflected,

I like how you have that at [corporate CDC]. There has been a corresponding loss with this incredible gain of having Inventing Remida upstairs on the third floor [in our $\mathrm{CDC}]$, in that we no longer have a reuse space downstairs. Ann's studio is a public area and has a visible emphasis on reuse, recyclable materials, loose parts and a lot of three-dimensional kinds of work. Our library used to be our reuse space and there is something great about having that publicly stated value and invitation for families.

Now that I have presented narrative data from the directors on creative reuse space for professional growth as Remida-inspired educators, I turn to the narrative session that followed two months later with the classroom teacher dyad.

Classroom teacher dyad. The dyadic pair took a moment to decide who would go first in responding to the second prompt: "Please share the story of how you came to 
be Remida-inspired?" Miranda asked politely, "Do you want to share first?" Clara accepted and began reflexively considering her own experiences and impressions, I wanted to go Remida and I wanted this awareness to keep going because it is healing, it is a problem that has been transformed into a possibility. That is the biggest inspiration for me, something negative that has been transformed into something positive. My deepest impression is the possibility being created through this project, through the Remida project.

Clara used the metaphor of "eye-opening" to continue to describe her experience of how the Inventing Remida Project inspired her classroom to create a vision to decrease the generation of waste materials. Clara said, "It is very thoughtful and a huge cause, it is an eye-opening experience not just for educators but for the children and parents who have been contributing to it." She elaborated on how Remida helps children, families and educators to understand the life cycle of a material—what materials are made from, where they come from, how they can be reinvented, and where they go when they are thrown away.

Then, Clara expressed her experience of connecting with the materials and seeing herself through the materials. She used the metaphor "transformation" to articulate her experience of consolidating and renewing her social identity and broadening the scope of identity studies from physical to relational through Remida materials:

Clara: And how have I come to identify myself as a Remida-inspired educator? By exploring the space and asking children questions - I think it is so thoughtful when children say how the materials have arrived to have a life, to be transformed 
into something else. We are sending a message through these materials because they are screaming possibilities that invent transformation. That is how I find myself-[Laughing] to transform and to create, that is how I find myself as a Remida-inspired educator.

Miranda: I love that perspective.

As a teacher, Clara identifies with Remida materials, as having the same affordance of transformation and creation.

Whereas both the studio teachers, directors and Clara had been on a study tour of Remida in Reggio Emilia, Italy, classroom teacher Miranda had not yet been. Miranda used the metaphor of a spark to describe her experience of becoming Remida-inspired through studio teacher Maggie,

Miranda: I haven't been to Reggio yet! I am at the beginning of identifying as Remida-inspired. Ever since I have been at [corporate CDC], Maggie has been very invested in reuse materials, loose parts and that idea of giving a material a life after its original use; she brought that spark back with her to our school from her trips to Reggio.

Despite Miranda's excitement she attributed to the spark that Maggie brought back from her experience visiting Remida in Italy, she also acknowledged that there is more work to be done to help children understand the nature of the reuse materials. Miranda: I feel there is a disconnect - we have a small space with materials available for reuse, yearly we ask families to donate in bulk and it is always available for donations. But outside of that one experience where the children are 
opening the bag of garbage or loose parts, I don't think we are doing a good enough job of bridging the gap of "Where do these come from, what's the purpose, why do we have them?" They see them as exciting materials to use in their play, but I could do more justice to the environmental piece.

As I reflected on the interview data over time, I realized that Miranda brought a unique layer to the narrative project. I began to interpret her central interview idea of "Bridging the Gap in Early Childhood Education for Sustainability (ECEFS)" as substantiating an image of the child as a global ecological citizen, capable of understanding and expressing more complex features of social identities.

Miranda narrated her experience of working with alternative and reclaimed materials as part of her professional practice and used the metaphors of hitting a "juncture" and a "little bump" to describe situating herself along a pedagogical spectrum of impermanence,

This year we have explored loose parts in a deep way in our classroom and in our school. We hit a juncture - a little bump — when children want to take their work home, tape it down [Laughing] or create something that is more long term.

Where is the balance between this idea of Remida, reuse, and recycle, and children's creativity, project work and creation?

Miranda's question raises significant issues regarding how to approach children's desire to keep the materials and constructions they create with Remida-inspired approaches which look at the constructions like Buddhist sand art for example, that is meant to teach something through its impermanent nature. Like studio teachers Ann and 
Maggie, classroom teacher Miranda seems in the process of locating herself as a Remidainspired educator along a spectrum of impermanence in her practice.

Similarly, to her dyadic partner, Miranda described her experience of Remida inspiration helping her classroom create a vision to decrease the generation of waste materials. This vision is facilitated by researching the life cycle of a material with children, families and educators; to understand what they are, where they come from, how they can be given a new life and identity, and where they go after they are thrown away.

Miranda also shared her hope for a stronger connection and more dynamic relationship between the laboratory and corporate CDC Remida-inspired centers. "I have a dream that this place (the IRPP center), and this vision could blend with our school in a more fluid way." Miranda's dream of a fluid connection with the IRPP is congruent with tasks in stage III renewal. During renewal, educators are particularly receptive to experiences in local workshops, and they profit from membership in professional associations and participation in their meetings (Katz, 1972). IRPP is a project that includes Remida cohort evening classes for educators and an advisory committee. Her dream echoes many of the participants' wishes for a reuse ethic that would permeate their culture in a more systemic manner.

Having presented key stories of constructing creative reuse cultural education center's through local and global ecological collaborations as Remida inspired educators, it is time to turn to the conceptualized data patterns of consolidation and renewal stage framework (Katz, 1972). 


\section{Data interpretations two: Consolidation and renewal stage growth}

\section{patterns-The Global Homesteader.}

Through a close examination of the second narrative inquiry prompt data, three patterns came into focus accompanying the archetype of the Remida-inspired educator as a global homesteader: (a) Plowing Remida as a geographic terrain and a terrain of consciousness, (b) Sowing Early childhood education for sustainability, and (c) Cultivating theoretical materials. In the following discussion, I describe these patterns in greater detail. To highlight collective patterns, I focus on participant responses to prompts from various data sets.

I interpreted the first data pattern to emerge through the homesteader lens as "Plowing Remida as a geographic terrain and a terrain of consciousness." By asking interview partners to share with one another the story of how they came to identify as Remida-inspired, I reached a deeper understanding about their perceptions of the interrelations between concepts of self and other in local and global ecological contexts. Like Borderlands cultural theory that emerged from Chicana studies, the Remida approach was described by multiple participants as being both geographic and metaphoric.

Keating (2009) describes Borderlands theory as follows:

For Anzaldua (1987), Borderlands, with a capital B, represents a concept that draws from yet goes beyond the geopolitical Texas/Mexico borderlands to encompass psychic, sexual, and spiritual Borderlands as well. These B/borderlands - in both their geographic and metaphoric meanings - represent 
intensely painful yet also potentially transformational spaces where opposites converge, conflict, and transform. (p. 318)

Similarly, Remida represents a theory that draws from (yet goes beyond) the geographic terrain from which it originates. This theory encompasses an ecological and socially sustainable mindset as well. As a related example, in response to the second narrative inquiry prompt, classroom educator Clara described her impression of Remida as both a physical terrain and a terrain of consciousness and her experience of broadening the scope of identity studies from physical to relational, It is positive if I start every morning working with Remida — not just the setting itself and how the materials are displayed but with the whole meaning behind it: we don't need to buy more stuff, we don't need to follow cultural consumerismletting children know materials would have been garbage if they hadn't of been reused on these projects, and that it is a lot of trash that would have been wasted. A social identity approach of acknowledging the interdependence of self and social context is present in global ecological citizenship narratives. In dyadic session one, Remida is viewed by Clara as a vehicle for an internalizing heightened awareness of human being's interdependence with each other and with materials. I interpreted this awareness as what Freire termed conscientizacion or critical consciousness. Anzaldua (1987) articulated conscientizao this way, It is only when she is on the other side and the shell cracks open and the lid from her eyes lifts that she sees things in a different perspective. It is only then that she makes the connections, formulates the insights. It is on then that her 
consciousness expands a tiny notch, another rattle appears on the rattlesnake tail and the added growth slightly alters the sounds she makes. (p. 71)

Clara also described this heightened awareness as "eye opening" in terms of cultivating a sense of global ecological citizenship that connects the self as being part of a greater whole, "It is very thoughtful and a huge cause, it is an eye-opening experience not just for educators but for the children and parents who have been contributing to it."

This description aligns with the idea that global ecological citizenship is about commitment to justice and wellbeing for all, in the neighborhood as well as on the global scale; global citizenship is about interrelation between self and other in local and global contexts (Dahlberg \& Moss, 2005; Duhn et al., 2010). Critical engagement with complex global issues such as ecological and social sustainability generates new understandings of how ECE can contribute to theory and practice of Early childhood education for sustainability (ECEFS).

As stated in the data, director Julienne describes her experience of broadening the scope of identity studies from physical to relational. Julienne's experience of sourcing materials and Remida cultural education as a concept that draws from, yet goes beyond, the external geographic place to encompass a relational mindset as well,

Early childhood teachers often wonder how to reuse materials for conservation of financial resources but Remida was a different mindset - the mindset of sourcing it so that there would be a community partnership.

As well, during the second narrative inquiry prompt, classroom teacher Miranda described adopting a Remida consciousness through her relationship with her Remida- 
inspired studio teacher and her hopes for someday traveling to Italy for a study tour. She shared that "Maggie has been very invested in reuse materials, loose parts and that idea of giving a material a life after its original use; she brought that spark back with her to our school from her trips to Reggio.” Relationship based education is integral to Reggio Emilia educational system, in which adults and children learn in a complex web of interdependencies (Project Zero and Reggio Children, 2001). This idea of relationallybased education and work show up time and again in the narratives put forward by participants like Maggie, suggesting that Remida is also about relationships more than about materials. Consumption is a complex social phenomenon that requires interactions and collaboration. Even research shows that the more humans are disconnected from their culture through the loss of cultural diversity via cultural homogeneity, the more global consumer culture increases (Firat, 2013). The participants share that Remida is creative reuse cultural education that is relationship-based.

Director Julienne described her experience of creating community partnerships through the process of sourcing materials. This experience of establishing a creative reuse cultural center, like the other participants, harken traditional farmer community cohesion reinforced through "raising a barn.” Julienne recounted,

We source materials from parents in the beginning of the school year and sometimes in the middle. During a big project, we will ask families for certain things like extra shiny discards: Can you save them? Can you look? Do you know anyone who can source them for us? Reuse materials are collected from homes and circles of relationships. 
In response to Julienne, Director Joni noted that the transparency of the reuse effort is important to the success of building community support for the Remida philosophy and project. She said,

Ann's studio is a public area and has a visible emphasis on reuse, recyclable materials, loose parts and a lot of three-dimensional kinds of work. Our library used to be our reuse space and there is something great about having that publicly stated value and invitation for families.

Further, the experience of networking with families and the greater community to source the materials and build on the Remida philosophy as the program grew over time was viewed by the participants as an important part of their own professional growth journeys. For example, during the first narrative inquiry prompt, Ann used a journey metaphor to describe how her Reggio inspired professional formation became stronger, consolidated, and renewed though relationships with Remida-inspired early childhood university programs, colleagues, supervisors, literature, professors, conferences, and her CDC.

After exploring the first pattern on plowing Remida as a geographic terrain and a terrain of consciousness, a second data pattern emerged through the homesteader lens as "sowing the seeds of early childhood education for sustainability (ECEFS)." During Katz's (1972) stage II of professional growth, an educator is likely to be ready to consolidate the overall gains made during the first stage and to differentiate specific tasks and skills to be mastered next. According to Katz (1972) the need for information about specific children or problems that young children present suggests that learning to use a 
wider range of resources would be timely. Exchanges of information and ideas with more experienced colleagues may help an educator master the tasks of this stage.

Opportunities to share feelings with colleagues in the same stage may help to reduce some of the sense of personal inadequacy and frustration.

Maggie articulated other participants' experiences when she shared the adversity of miseducation around conservation,

Becoming Remida-inspired developed from my frustration as a parent and teacher about sheer volumes of waste. Children develop a mentality of having ideas, wanting to buy materials; "I need more, I need more!" I felt frustrated by social things, like a child putting one mark of paint on a paper and then throwing it away. I would suggest someone else might want to add to the drawing, but the child would respond, "No, it's mine!" I experienced frustration with the way resources were used and it was building to a high level prior to my first trip to Italy. Prior to the trip, you choose your itinerary. I was reading about the Remida center in the schedule of events and I thought to myself "Yes, this is what I want to know!" I signed up to go and I was blown away!

This is what we want to be doing, what we don't have words for, that we can't explain.

All the participants shared that they were instinctively drawn to Remida Early childhood education for sustainability (ECEFS) and like social constructivism, it just made sense to them as a solution that they had been seeking but had trouble finding in their professional formation. Many of the participants viewed a creative reuse approach 
as common sense and experienced the phenomena of feeling like they recognized Remida once they saw it. This recognition of Reggio-inspired Early childhood education for sustainability (ECEFS) was like experiences of recognizing the common sense of the Reggio approach altogether earlier in their career. The initial experiences of Reggio Emilia approach to education were more along the lines of recognizing the unnamed such as when Maggie said, “This is what we are doing, what we don't have words for, that we can't explain." Whereas, initial experiences of Remida were more along the lines of a statement made by Maggie, "Yes, this is what I want to know!"

This distinction is important to note because during Katz's (1972) stage II of professional growth, an educator is likely to be ready to consolidate the overall gains made during the first stage ("This is what we are doing, what we don't have words for, that we can't explain.”) and to differentiate specific tasks and skills to be mastered next, such as becoming Remida inspired ("Yes, this is what I want to know!"). Through the homesteader lens participants described sowing the seeds of ECESF with Remida inspired pedagogy that will bear the theoretical fruits their child development centers value the most. Over the years, they try experimenting with planting different seeds to compare how well those ideas and provocations take root and help the community to thrive as an interconnected, interdependent whole.

Now that we have explored the first two patterns of stage II and III professional identity formation in becoming Remida-inspired as a geographic terrain and a terrain of consciousness, and as sowing the seeds of early childhood education for sustainability (ECEFS), I will turn to the third pattern that emerged from the data- the idea of 
educators developing strategies for finding, filtering, and selecting extra open-ended reuse materials.

I interpreted the third data pattern through the homesteader lens of "cultivating theoretical materials." During Katz's (1972) renewal stage of professional formation, educators widen the scope of resources they draw upon to expand and refine their classroom practice. They actively draw on reading numerous journals, viewing films and video, and using the Internet as sources of fresh ideas. Many participants in this study narrated their experiences of struggling to find the resources for the ecological approaches that they valued as early childhood educators.

For example, in the first dyad session, studio teacher Ann related her former experience of struggling to find the language for the ecological approaches that came intuitively to her as an individual educator. She found value in Remida presentations "because the materials are interesting to look at and because of how they phrase things." She further emphasized this point by stating that during Remida presentations she must remember to take notes “because I think like them, but I don't know how to express it like they do; most the things they say are quotable!" In other words, opportunities to learn from experienced Remida practitioners provides language and resources that are useful for evaluating and selecting useful materials that align with the goals of the Remida-inspired pedagogy she is actively working to cultivate in her school and studio.

One of the pedagogical aims of Remida work is shown to provide children with reflective experiences that help them to understand nuances of human ecology as part of the broader environment (Reinisch \& Parnell, 2006). As another example during the first 
dyadic session, studio teacher Maggie articulated that her use of the lens of social constructivism over the past decade heightened her appreciation of the complexity of interrelatedness and her growing awareness of the role materials and the environment have in human ecology. She said that the biggest thing she has learned is "How important children's relationship with me, with their families, and with materials is." She continues, "That idea really grew for me, that children should learn not only in relationship with each other, but in relationship with materials, and with the environment, and with me, etc." Importantly, she did not express this as a static understanding of relationships but rather one that grows and changes. Maggie cultivates Remida inspired theoretical materials as her sensitivities expand through experience. Similarly, she recognized that as a Remida inspired educator, she is not alone in this constantly shifting perspective.

For studio teacher Maggie and classroom teacher Miranda (who both described identifying as being Remida-inspired prior to going to international conferences and workshops in Reggio Emilia, Italy), collaborations with Remida-inspired colleagues were the first step toward articulating their theories about the relationship between identity and reuse materials. The experience of working with others to figure out how to articulate this framework was facilitated through experiences that intentionally kept situated cognition at the forefront of their minds and relationships formed within their ecological contexts. In response to the second narrative inquiry prompt: "Please share the story of how you came to be Remida-inspired," director Julienne shared, "A big part of working with the materials after that was to get them out of the closets, accessible to the 
community and to see what this kind of commitment or value [they] would bring to our program." To elaborate, Remida-inspired educators cultivated theoretical frameworks with materials through a) working as co-researchers with children, adults, the environment as the third teacher, and b) with community resources that provided novel ways of analyzing their perceptions and shifting their approaches in the studio and classroom.

This global homesteader growth pattern is important to note because similar to the previous growth pattern of sowing the seeds of Early childhood education for sustainability (ECEFS), during Katz's (1972) stage II of professional growth, an educator is likely to be ready to consolidate the overall gains made during the forager stage ("This is what we are doing, what we don't have words for, that we can't explain.”) and to differentiate specific tasks and skills to be mastered next, such as becoming Remida inspired ("Yes, this is what I want to know!"). Cultivating theoretical materials is a specific task and skill that the participants differentiated in their practice.

To summarize the main points made in this subsection, what follows is a re-story of the narrative data that combines six participants voices into one protagonist's story, told as a re-story. Once more, specific metaphors used by the participants are italicized in the re-story and the story is written single spaced to honor the narrative style. The purpose of this technique is to share the group of participants' experiences of feeling conflicted about the lack of Early childhood education for sustainability (ECEFS) and the resolutions to this problem that they are developing through socially identifying as Remida-inspired in their practice. 


\section{A re-story: The Global Homesteader.}

A group of educators that started Remida-inspired work at our school traveled to Reggio Emilio, Italy to work with the Remida Center where they dug into the cultural education and creative proposals inherent to the approach. Another teacher in my center was very invested in reuse materials that she referred to as loose parts. She was excited about the idea of giving a material life after its original use and she brought that spark back with her to our school from her trips to Reggio Emilia and Remida.

When I first visited Remida in Italy, I had an intense feeling of familiarity, of coming home, of this makes sense to me. We decided to build a creative reuse cultural education center at our school and wanted to harness collective resources and not to do it by ourselves. We got started by talking with a non-profit reuse organization called SCRAP who shared the same mindset. Then our footprints, our ideas, were shaped by undergraduate capstone students who did some groundwork for us as part of a reuse project class assignment.

At first, people tried to make creative reuse this big separate project. Now years later, it is in the veins of our school-it flows through the veins. We have integrated the value of learning how to transform our program. That is how I find myself as a Remida-inspired educator.

We had already captured the piece about aesthetics and wonder, and what the materials might tell you. By now we have learned how to integrate experiences of learning along a spectrum of impermanence into our everyday teaching practices and find this to be important. Remida discards support ideas or projects in the classrooms; we might beef up one collection or type of material that has attributes or affordances to support the work. Remida materials are extra-open ended for children to use them. We are sending a message through these materials because they are screaming possibilities to invent transformation. It is very thoughtful and a huge cause, it is an eye-opening experience not just for educators but for the children and parents who have been contributing to the process of practicing the Remida approach in adaptive ways that fit with the context of our school community and identity.

We are continually developing an epistemology of display because you also see where your values are crumbling if it gets disorganized or misused or not tended to because it is right there in the front of our school. In fact, if we are not diligent in our stewardship of the Remida approach it can become an eyesore rather than something viewed as a shared community asset. 
This year we have explored loose parts in a deep way in our classroom and in our school and we have hit a juncture - a little bump — when children want to take their work home, tape it down or create something that is more long term. We are asking ourselves where is the balance between this Remida idea of reuse and recycle not just once but again and again and children's pride in displaying and sharing the creativity expressed in their project work on a longer-term basis?

In response to these and similar questions, we find that we are fine tuning our educational philosophies related to what it means to teach children about the finite nature of resources in our environment through creativity and play versus through fear. As a community of practice, we realize that we still have work to do to figure out how to integrate the Remida philosophy in a more systemic manner throughout our research practices. For example, often when the children are opening bags of loose parts, I don't think we are doing a good enough job of bridging the gap for children by prompting them to examine questions such as, where do these [reuse materials] come from, what's the purpose [of these materials], and why do we have them?" In fact, I have a dream that the Inventing Remida Project vision could blend with [the corporate creative reuse cultural center] in a more fluid way.

\section{The Global Homesteader summary.}

The re-story above allows us to look at Remida-inspired educator formation

through the archetype of The Global Homesteader. Formerly, we also explored a re-story with The Forager archetype. This participant group described Remida-inspiration as actively engaging in an iterative, reflective process as a broad community involving educators both within and external to the school, children, parents, and other community partners.

Participants used the metaphors spark; coming home; dug into; harness; groundwork; footprints; captured; beef up; transformation; flows through the veins; hitting a juncture; little bump; next layer; fine tuning; and dream, to describe their experiences of becoming Remida-inspired educators, which led me to interpret their early professional formation through a global homesteader archetype. The global homesteader 
as a metaphor corresponds with Katz "consolidation and renewal stages" of teaching. In these second and third stages of professional growth, the educator's overriding concerns are challenging situations and professional renewal. More specifically, the early childhood educator who is in the consolidation stage has usually come to see themselves as capable of survival, is ready to consolidate the progress they have made, and to differentiate skills and tasks to be mastered next. Reflecting on the narrative data led me to see patterns of professional formation that led to the adoption of Remida pedagogy.

Global homesteaders see that, in the long run, the best way to sustain their Remida- inspired reuse practice is to be self-sufficient as a community. They get to a place in their professional development as individuals and as a learning community where they want to settle down and work towards the goal of creating a metaphorical homestead for creative reuse cultural education.

Further, I elaborate on the global homestead as a symbolic image for participants creation of a Remida-inspired center in their schools. To plow a garden (or a creative reuse cultural education center) the participants described needing to have a vision. When participants were planning their reuse cultural center's, they started by creating a community wish list of everything they wanted to grow there, metaphorically speaking. However, they eventually trim down their list to the seeds that are the most important to sow. They have a limited space, and can't possibly cultivate everything at once, otherwise, they end up with a huge mess that would be unmanageable to steward. They sowed the seeds that will bear the theoretical fruit that they value the most. Then, over the years, they tried planting different seeds. 
As homesteaders, they spend a lot of time, energy, and expense to improve the soil of their plot and to create a strong, rich, durable production. They describe having done this by adding the compost and nutrients of relationality, theoretical frameworks, situated learning, early childhood education for sustainability, and theoretical material as organic fertilizers for a Remida-inspired environment that is conducive to social and ecological sustainability.

The participants as homesteaders created small scale Remida-inspired education centers in their respective laboratory and corporate CDCs that renew their learning communities. The participants describe working towards creating a sense of community, because it ensures their chances of pedagogical survival. They continue to learn Remidainspired skills through colleague advice and consultants as well as conferences, professional associations, journals, and visits to Remida Italy. Instead of finding chickens, pigs, a garden or a green house on their homesteads, one finds unique paper, colored glass, broken tiles, textured fabric, smooth metals, various buttons, colored plastics, interesting containers, misprinted stickers, wooden pieces, jewelry, beads, scraps of leather, colored lids and caps. The homesteaders aim to grow as much of their own creative reuse as possible, with their own hands, their own tools, and their own time. There is a degree of inter-connectedness, and a desire to build a strong homesteading community, that is intentional.

Now I turn to the narrative data collected on the final maturity stage of professional growth, the following subsection employs the metaphorical archetype of the navigator to describe the stage IV of professional maturity. 


\section{The third narrative inquiry prompt: The Navigator.}

The third prompt, "Please share what identity studies means to you, and how you have approached identity studies with young children as a social constructivist educator" was designed to better understand participants' experiences of working with reuse materials and identity studies through beginning to articulate their belief structures about identity, cognition, and reuse.

In the third dyad sessions, participants used the metaphors fluid; infused; full range; flipping; anchoring; work backwards; imbedded; uncover; broadened scope and evolving to describe their experiences with Reggio inspired identity studies. Although each participant's experiences were unique, patterns of approaching identity studies as social constructivists came into view which I interpreted through the symbolic image of the navigator.

In the dyadic narratives that follow, metaphors emerge among the participant pairs that describe their shared recognition that exploration can be relational as well as physical. Participants expressed that a common lesson to learn was to take the initiative while coordinating with others to break barriers and accomplish great things. A mature educator possesses a broader perspective to begin to ask deeper, more abstract questions, such as "What are my historical and philosophical roots, what is the nature of growth and learning, how are educational decisions made, can schools change societies, and is early childhood teaching really a profession?" Perhaps they have asked these questions before, but with experience, the questions represent a more meaningful search for insight, perspective, and accuracy (Katz, 1972). This Navigator archetype corresponds with the 
maturity stage, where the overriding concern is addressing the larger picture and the primary resources needed are seminars, institutes, degree programs, books, journals, and conferences.

Studio teacher dyad. Focusing in on Ann and Maggie's session together, I find new metaphors in their stories that yields data that are both unique-to-the-individual and universal-for-the-individual symbolized here as the archetypal navigator. The pair turned to the third narrative inquiry prompt: What does identity studies in early childhood education mean to you, and how have you approached identity studies with young children as a social constructivist educator?

At this stage in the first session of dyadic interviews, the third prompt seemed to elicit deeply reflexive considerations of impressions that each pair shared at length. Maggie narrated her experiences of exploring children's identities through the inclusive lens of the term exceptionalities and used the metaphor "fluid" to describe her perception of children's identities as compared to adults. In her dyad session, she states,

We did a lot with our infants and toddlers last year through inclusion-based attention on trying to identify children's exceptionalities and I would identify those as fluid; they might show a real strength in one moment and then a completely different one a moment later. Children's identity is more fluid than ours and is not so set at that age.

Over time, as I reflected on the interview data, I realized that Maggie brought a unique layer to the narrative project. I began to interpret her central interview idea-children's identities are more fluid — as substantiating an image of the child as a competent global 
ecological citizen, capable of understanding and expressing more complex features of social identities.

Moreover, Ann identified influences on educators' approaches to identity studies with children, and located how she constructs meaning along historical and social constructivist continuums as she shared,

When I was first teaching, we talked about how we were all the same; we might look different on the outside, but we are all the same on the inside. Now the message is that we are all different, and that is great. That is a major shift that has happened over the years - not just in my practice, but in what I have heard from other educators and in workshops.

Ann narrated a short story involving a basket in the reuse center to illustrate her experience, "That is how I would say that I approach identity studies and children, sometimes intentional with projects and sometimes just by having that heightened awareness." I interpreted this awareness as what Freire termed conscientizacion or critical consciousness. Ann says,

I try to be aware of opportunities to discuss gender with children, their own identity and their ideas about identity. When we were making little wood dolls, for the lands that we are making out of reuse materials, it was toward the end of the project and one of the girls said to me, 'So is this the basket for the girls, and this is the one for the boys?' I could have dismissed it [Laughter] but one basket had different colors of fabric in it and the other one had lace, ribbons and sequins. 
One basket had all the major colors, and she perceived that as the boy basket. The other basket with the ribbon and lace, she perceived as the girl basket. Rather than saying 'Oh, no, those are for everybody' [I recognized that] it was an opportunity to stop and ask her 'Tell me what you are thinking about here? How come you think that is the boy or the girl one?' and to have a conversation with her. Ann then used the metaphor of infused to describe her experience of traversing a multiplicity of identity studies,

Our school is good about making sure that identity studies are infused into the curriculum throughout the year, every year. Some years it has been an intentional study, like what you have up on the wall [pointing to atelier documentation]. We help children more intentionally with making faces, and buddy representations where children represent each other on felt faces, clay, reuse material, and painting images of themselves and each other with skin colors. Other years it is more infused; we weren't intentionally studying identity, but identity comes up. Being aware of it as a teacher and being able to address it is important to me. As Maggie and Ann's narratives illuminated that social constructivist ECE is identity studies, I came to understand that Reggio Emilia-inspired education is identity studies. Further, during the studio teacher interview, I asked the dyad an additional question: How has Remida enriched your approach to identity studies? Ann and Maggie responded to this question by saying it is interesting what adults focus on when making self-portraits compared to how a child focuses on having a nose and a mouth. Anne noted that when she makes identity dolls for the 'fantasy lands' that the children construct 
out of reuse materials, they might grab one googly eye and one button eye, "I ask, 'What is that one?' and they reply, 'It's their eyes,' And I say, 'Oh, okay!' and I continue, 'Don't you want to use two buttons?' [Laughter] “Or two googly eyes?” Maggie agreed it is important not to correct children when for example, they are studying color and are saying "My skin is green." She felt it is important to understand what is more important to them in the moment and not get into a tussle over "No, your skin is not green, look this is green!" Maggie added that often parents don't want their boys to wear dresses or they can't understand why their son's wanting to wear nail polish and stated, "I believe in letting children self-identify and not arguing with them in order to understand how fluid identity is and how that changes over time. When they are 15 or 16 or 25 , they are not going to still think that their skin is green.”

Additionally, Maggie elaborated on theoretical material as follows: Open-ended materials, loose parts and Remida materials of course, play into those kinds of conversations with children because children's toys are often gender specific_-specific colors, specific tasks that don't allow for freedom of thought or the evolution that children need to go through. Remida materials allow children flexibility of thought and that supports a million different things, but it really supports identity studies.

The theory that emerges from within Maggie's identity work affirms that preschool age children are capable of understanding and expressing more complex features of social identities and that extra-open ended Remida materials support navigating the fluidity of identities and constructivist identity studies. 
The conversation between the studio teachers flowed into a reflection on the affordance of Remida materials being forgiving because of their imperfection. For example, Ann described that during the past two years, her school has held an impermanent reuse self-portrait event for the whole school on the laboratory CDC's "Grandparents and Special Friend's Days." During this event entire families made representations of each other and themselves. She shared that "parents made little mustaches and beards, put earrings and accessories on. It was wonderful to see people representing their whole family and a joyful playfulness amongst them." Ann observed that there is something about the materials that seems to take the pressure off people to create perfect self-representations. "There is something special about Remida materials because you know they are going to be imperfect" concluded Ann.

Maggie enthusiastically responded to this observation stating, "There is no wrong answer! By our cultural standards, you are either a painter or not but with an open-ended reuse material, there is no wrong way to do it." Ann replied that while there are some people who are artistically talented, it is also amazing to observe what a 2-year-old can create alongside their parent. Maggie added,

When I paint myself, I purposely go 'Picasso'—abstract, because it is going to be bad if I try to do it well, so I go cartoonish, the other direction, painting purple skin, because I feel the pressure. That is where the joy comes from, the pressure is gone; you are not going to do it badly with these reuse materials.

Both studio teachers observed the sense of joy participants experience when working with reuse materials to express themselves. They interpreted the sense of joy 
and absence of pressure to perform as positively influencing people to use their imaginations.

Later, when the studio teacher dyadic pair turned to fourth interview question in session two a paradoxical affordance of the reuse materials emerged. The interview question asked of each dyadic participant was: What is difficult, challenging or rewarding about working with reuse materials with children and how does that affect you?

Ann noted, "There is a confidence level with how to introduce Remida materials. A lot of teachers don't know what to do with the reuse or loose parts material." Maggie responded, "That is not necessarily any different from any other material. I find reuse to be not a hundred percent approachable but more approachable." Ann observed that the reuse self-representation activity is one of the few when teachers say "Can I borrow those? Do you still have those?" and they say, "Children are still talking about them, can we borrow them?" I wondered aloud about how it is that children requested them more than the teachers. I followed up, "Was it because they remember doing them in the studio?" Maggie replied, "Yes, this is concrete provocation. Sometimes the teachers would ask 'How do you know those materials are going to go together?' And the answer is, because you are thinking as you are designing it, for example — what sounds do these make?"

Maggie emphasized that touching the materials helps teachers notice some material fits inside of others, but not inside of some. She went on to explain that some children wish to explore which pieces to take as they start to build; they are touching and gathering materials throughout. Maggie concluded, "You learn by doing." Ann agreed, 
"You have that process - that sub-process - the teacher's need that level of scaffolding for themselves as well." Here, the idea of teachers working to find their roles in relationship with other adults, the children and the materials makes visible the ongoing need to navigate the paradoxical affordance of the materials.

The notion that arises out of Maggie and Ann's consideration of the third narrative inquiry prompt is that Remida inspired materials are paradoxically both forgiving and demanding.

Now that I have presented key studio teacher ideas of individual's experiences with socially constructing identity studies, I turn again to the narrative session that followed two months later with the director dyad.

Director dyad. As we focus in on Joni and Julienne's session together, we find new metaphors in their stories that yields data that are both unique-to-the-individual and universal-for-the-individual symbolized here as the archetypal Navigator. Joni used the analogy of "a full range" to describe a multiplicity of identity studies. She emphasized how historically, identity studies are a value in critical approaches to ECE and how she started paying attention to identity studies through anti-bias work. This involved looking at the Derman-Sparks and Edwards (2010) anti-bias goals including that each child will: Express positive social identities; voice comfort and joy with human diversity; recognize and speak up about unfairness; and demonstrate the courage and the ability to act, with others, or alone, against prejudice and/or discrimination.

Using navigational metaphors, Joni stated, "There is a full range of identity studies whether that's self-portraits or portraits of each other." And then the metaphor of 
"anchoring" to describe her experience of directing the route or course of identity studies in her learning community as more of a social than an individual provocation, "Identity studies is a way of valuing and anchoring a connection to the bigger whole." The directors' reflections were aligned with stages of maturity when an educator possesses a broader perspective to begin to ask deeper, more abstract questions about the historical and philosophical roots of identity studies, theories of growth and learning, and how cultural education can change societies. This alignment is evident when identity studies is intended as a means of valuing and anchoring a connection between the individual and the social; between the local and global.

Julienne used the metaphor of "imbedded" to describe her experience of guiding identity studies as an enduring question and value statement in her Remida inspired corporate CDC. She shared how her learning community made a "big shift" from goal statements to value statements influenced by Reggio's written document on the Rights of Children: That children have the right to be recognized as citizens with individual, social and legal rights. Julienne stated, "We started thinking about our school being a place where you come to understand yourself better so that your ideas can go out in the world." Julienne continues by describing that when her teachers construct enduring understandings and questions as value statements, they talk a lot about identity which was then imbedded in almost every value statement.

Director Julienne used the analogy of "work backwards" to describe her steering approach, and then the metaphor "uncover" to describe her piloting of social constructivist approaches to identity studies, 
We work backwards from "Who are we together?" A lot of our children can't speak for themselves — what are their wishes, hopes and dreams? How do we uncover that? All that you hope a school can be is that it inspires individuals to carry within and outward how they think about themselves.

Now that I have presented key director ideas of navigating identity studies as Remida-inspired educators, I turn to the narrative session that followed two months later with the classroom teacher dyad.

Classroom teacher dyad. Miranda and Clara's time together centered on the third narrative inquiry prompts: What does identity studies in early childhood education mean to you, and how have you approached identity studies with young children as a social constructivist educator? Clara also narrated her experience of identity studies as an individual and social proposal to children. She described explorations of Who am I? What is my role? How can I contribute to my community? And how do we relate to one another? And she exclaimed, "There is so much to discuss and to think about, to wonder and to explore. This idea of self and sense of self- - how it relates to others, how do we connect with others? It is beautiful, it is powerful!" There was a quiet pause of agreement and nodding heads between this dyadic pair of classroom teachers.

Miranda then used the analogy of "evolving" to describe her ongoing experience of a multiplicity of identity studies. She clarified how her corporate CDC holds identity studies as important for each school year and as a provocative curriculum to 'jump start' the year and think about-Who am I? Who are we? And what is it going to look like for us to be together this year? She explained that identity study projects are yearlong. She 
expressed that as they think about negotiated curriculum and identity studies, they find that identity studies are "just ever evolving and new things are popping up weekly that we are discovering about the children and that the children are discovering about each other. We are constantly evolving, changing and growing together." And how having awareness and a value on social identity has brought the opportunity for deeper connection, relationships and appreciation for each child's uniqueness.

Miranda used the analogy of "broadened their scope" to describe her experience of going from the physical to the relational with identity studies:

You were talking about going from the physical to the relational, and I find that adults and children do that also; they think about the physical first. I found that the questions we asked them as we were exploring identity either got children thinking about the physical or broadened their scope.

As a navigator, the mature early childhood educator is likely to have come to identify themselves as an educator and to have reached a comfortable level of confidence in their own competence. This idea is shown in the key data described and is explored more in the interpretations next.

\section{Data interpretations three: Mature stage growth patterns-The Navigator.}

Through a close examination of the data, three patterns came into focus accompanying the mature stage Remida-inspired educator who approaches identity studies as a Navigator: (1) Broadening the scope from physical to relational; (2) Voyaging backwards from We to Me; and (3) Recovering a multiplicity of identity studies. In the following discussion, I will describe these patterns in greater detail. To 
highlight collective patterns, I focus on participant responses to prompts from various data sets as shared above.

I interpreted the first data pattern through the Navigator lens of "broadening the scope from physical to relational." The intention of the dyadic interviews was to create a space for participants to take a deeper look at their reuse materials experiences with toddler and preschool-aged children and to begin to articulate their underlying belief structure about identity and reuse. Multiple participants, including Miranda, found that the questions they ask children around exploring identity "either got children thinking about the physical or broadened their scope." Miranda shares how conceptualizing identities as a source of data in children's learning can reorient theories about the relationship between the individual and the social by calling attention to the social and cultural dynamics; dynamics through which individuals construct themselves as social actors. This concept is reminiscent of Sfard and Prusak's work (2005). As they remind us, proving space for peoples' inner forces to emerge in their narrative theory of identity is a powerful analytical tool in understanding the cultural shaping of learning.

Moreover, classroom teacher Clara described the physical dimension of identity studies as she states, "The children will be in front of the mirror with this whole idea of exploration about the question, Who am I? More in a physical aspect, How do I look to other people? And. How do they perceive me?" Seemingly, these early childhood educators who identify as social constructivists engage an appreciation of the influences of cultural, social, and economic diversity and variations of individual growth to inform identity studies with young children as reminiscent in the literature as Katz $(1992,1994)$, 
Reggio Children (1996), and Kissinger (2017). Even further, Maggie shared her navigation through the physical dimension of identity studies, I work with a diverse age group. Preschooler's often do what we call "partner drawings." They may begin with self-portraits and then draw each other. They are paired and so they are getting somebody else's feedback, like "You forgot your nose" or "That is not how my hair looks." Compared to older children who tend to think more abstractly, the older toddlers talk about the parts as they collect them. I am thinking of children who were using reuse materials and saying, "This is my eye." While pointing way down in the bottom left corner of their portrait, and then saying, "This is my other eye." And pointing to a completely different material placed off the canvas. Two-year old children are just starting to learn these terms - my eyes, my nose and ears, those kinds of things. Maggie then narrated her experience of broadening the scope of identity studies from physical to relational, We try to identify qualities in the moment that children are bringing to the classroom - perseverance, empathy, for example — through an act like helping someone else get their shoes on. Center wide, we tend to focus a lot less in our identity studies on physical appearance and more on trying to identify children's gifts or their exceptionalities together.

Katz reminds us that a mature educator continues to do the things they have done during the first two stages in interactive, cyclical patterns but they are more insightful at this stage as to how to recognize opportunities. They tend to recognize how to build on 
their own constructs and scaffold for children new ways of socially constructing identities.

Navigating identity studies in ECE to broaden the scope from physical to relational aligns with recent literature that refocuses attention from individual to social or collective identity. More recent discourses in early childhood identity theory trend toward the multiplicity of social and collective identities (e.g. Brooker \& Woodhead (Eds.), 2008; Konstantoni, 2011, 2012). Collective identity is the belief that through participating in social activities, individuals can gain a sense of belonging and an identity that transcends the individual; community building activities support positive identity formation. We can see these activities in Maggie's experiences as in helping someone and identifying gifts in children together.

I interpreted the second data pattern after the interviews were shared through the Navigator lens as "Voyaging backwards from We to Me." Discourses in identity theory warn of the risk of "endorsing the myth of the autonomous and Independent person" (Prout, 2011, p. 66) and ignoring that people — adults and children alike-live in a complex web of interdependencies (Gallacher \& Gallagher, 2008). Katz (1993) suggests that while everyone may be special, as educators we surely want to reinforce in children the view that, while we are unique in some respects, we also have a great deal in common.

Director Joni used the analogy of "flipping" to further describe how her laboratory CDC reversed identity studies over time and instead of beginning with individual identity, the studies begin with social identities, 
For years, we started our school year with "Who am I? Who are you? Who are we together?" and then we started talking about flipping it: "Who are we together? and Who am I and Who are you?" inspired by the Carla Rinaldi quote "My identity is a shared identity with others. I am who I am because of you." I have come to recognize that we are each created by our relationships and place in the community.

Emerging identity theories in sociological, postmodern, and social constructionist literature refocuses attention from the individual to the collective (Cerulo, 1997; Project Zero \& Reggio Children, 2001). All of the participants described trying to always keep identity provocations as a social question rather than, "How do you see yourself?" According to Bennett and Sanni (2004), developmental psychologists have almost exclusively treated groups as external to the self; they have viewed group dynamics as merely an influence upon children's self-concept. In contrast, a social-cognitive perspective and social identity approach forwards the idea that "at its heart is an acknowledgment of the interdependence of self and social context" (p. 1). One of the central insights of the social identity approach is that groups can become part of the selfconcept.

Studio teacher Maggie used the metaphor of a "nutshell" to synthesize her experiences of identity studies as a social provocation:

We make take home books where everyone adds a page about what they did over their weekend, so the child's identity is more about them but also their identity within their family, within their community, looking at it in a broader sense. It is 
this huge, huge thing so in a nutshell, we try to always keep our identity provocations as a social question rather than, "How do you see yourself?" As is the case with the narrative data I collected from Maggie, Ann, Joni, Julianne, Clara, and Miranda about their social constructivist approaches to identity studies as Reggio and Remida inspired educators, scholars (Brooker \& Woodhead (Eds.), 2008; Konstantoni, 2011, 2012) explore identity as a human construct, constantly created and recreated in interactions with people. The literature review in Chapter 2 of this study revealed a re-conceptualization in multiple disciplines around the concept of identity, including cultural studies and educational research such as in Sfard and Prusak (2005). Sfard and Prusak argued that providing space for peoples' inner forces to emerge in their narrative theory of identity is a powerful analytical tool in understanding the cultural shaping of learning. Their thesis is that the concept of identity itself is the missing link in a complex dialectic between learning and its sociocultural context.

Further, the third dyadic prompt takes us to interpreting the data through a Navigator lens. Interestingly, the word "nutshell" that studio teacher Maggie used to synopsize her approach to identity studies, has circulated since roughly 1200 and is used figuratively with reference to a copy of the epic journey Iliad that the navigator Homer used so small it could fit into the shell of a nut.

This Navigator archetype corresponds with the maturity stage where the overriding concern is addressing the larger picture and the primary resources needed are seminars, institutes, degree programs, books, journals and conferences. Participants stories aligned with the view that the best practice for identity studies in early childhood 
education is grounded in a social constructivist theory of learning. In keeping with this theoretical perspective, as Remida-inspired educators and researchers, the participants elucidate this sustainable approach to identity studies with reuse materials that socially constructs individual selfhood $m e$ in early childhood within the context of ecological selfhood we.

I interpreted the third data pattern through the Navigator lens of "re-covering a multiplicity of identities studies." Most Reggio-inspired educators believe that identity is a social construction, shaping and being shaped by the school. This study explores how Remida locates its practices in ECE spaces where dominant constructions of early childhood identity can be contested in creative ways that invite the co-construction of a multiplicity of identities. According to Katz (1972) mature educators welcome the chance to read widely and to interact with educators working on many problem areas on many different levels. This sentiment seems portrayed in the data. For example, Ann noted, "That is how I approach identity studies and children, sometimes intentionally with projects and sometimes just by having a heightened awareness." Ann's desire to interact and grow demonstrates how she navigates the terrain of reuse materials, multiplicity of identities and the we/me embedded in her approach.

As well, working with reuse materials to explore social identity issues is an example of empowering children's agency. Children carry and construct their own culture and are therefore active participants in the organization of their identity, their autonomy, and their capabilities. Creative reuse informs their thinking with others and how they perceive others to be in relationship with materials. Maggie reflected on 
fostering children's rights as global ecological citizens to work with complex materials, and the rights of the materials themselves to be given new identities. Maggie states,

Everything a child does expresses their identity and supports them in learning about who they are in the world. This year our intention is to research loose parts. This is one of our questions: what role do open-ended materials, specifically loose parts, play in inviting children to think creatively and problem solve in new and evolving ways?

Maggie's idea of the identity and learning supported through creative thinking and problem-solving illuminates a we-identity and seeks to foster social identity construction. This is similar to Ann's desire to interact and their overall interest in identities as an endeavor of we rather than something done alone or in isolation.

To summarize the main points made in this subsection, a re-story of the narrative data follows. The re-story combines six participants voices into one protagonist's voice. Once more, specific metaphors used by the participants are italicized in the re-story and it is written in single-spaced to evoke the spirit of the narrative voice. The purpose of this re-storying technique is to share the group of participants' individual experiences of navigating a multiplicity of identity studies, which comes to resolution through socially identifying as social constructivist in their approach.

\section{A re-story: The Navigator.}

Identity studies is a way of valuing and anchoring a connection to the bigger whole. When we socially constructed our enduring understandings and questions, which are our value statements, we talked a lot about identity, and it was embedded in almost every statement that we made. It is this huge, huge thing so in a 'nutshell,' we try to always keep our identity provocations as a social 
question rather than just how do you see yourself as an individual. I found that the questions we asked children as we were exploring identity and the way we framed those questions either got them into thinking with the nature of physical reality or broadened their scope to conceptual ideas. Our school is good about making sure that identity studies are infused into the curriculum throughout the year, every year. There is a full range of identity studies whether that's selfportraits or portraits of each other. Other years it is more 'infused'; we were not intentionally studying identity, but identity comes up.

For years, we started our school year with the questions who am I, who are you and who are we together? Then we started talking about flipping it-Who are we together and who am I and who are you? What we find out about one another each time is going to be different and keep evolving. A lot of our children cannot speak for themselves - what are their wishes, hopes and dreams? How do we uncover that? One child entered our classroom and we could tell early on that he marched to the beat of a different drum. For example, if he saw these two pens together, he would use them to pick up food; he has a very mechanical way of thinking and it enlightened us to broaden our scope to consider what is possible and who he is.

We did a lot with our infants and toddlers last year through inclusion-based attention on trying to identify children's exceptionalities and I would identify those as fluid; they might show a real strength in one moment and then a completely different one a moment later. Children's identity is more fluid than ours and is not so set at a young age. I believe in letting children self-identify and not arguing with them based on understanding how fluid identity is and how that changes. Remida materials allow children flexibility of thought and that supports a million different things, but it really supports identity studies. There is something about Remida materials that takes pressure off people. It is a release of pressure that requires you to pretend. That is where the joy comes from, the pressure is gone; you are not going to do it badly with these materials. Remida materials are discards, rejects, destined to be thrown away. When we reclaim them for identity work, we give them a new life, a new identity and a sense of belonging.

\section{The Navigator summary.}

Participants used the metaphors anchoring; imbedded; in a nutshell; broaden our

scope; full range; evolving; and uncover to describe their experiences with approaching identity studies as social constructivists, which led me to interpret their mature stage professional formation through a Navigator archetype. The Navigator can be considered 
a symbol of leadership, congruent with Katz fourth "maturity" stage where the overriding concern is addressing the larger picture and the primary resources needed are seminars, institutes, degree programs, books, journals and conferences. Reflecting on the narrative data led me to see patterns in becoming Remida- inspired that led to the adoption of reuse materials in identity studies.

The participants describe children's identities as being more fluid than adults and how extra open-ended Remida-inspired materials support constructivist identity studies. The participants explore social identity studies by trusting their intuition instead of having to control the course-so to speak. A navigator image is not to be mistaken with the Age of Discover - the period during which European nations sponsored expansion of their colonial reach to Africa, Asia, and the Americas. Instead, an indigenous and ancient method of navigation is summoned here as an archetype-reminiscent of seafaring cultures who 6,000 years ago used traditional navigation arts to guide their initiates across the Pacific without compasses or charts (Thomas, 1987).

I draw from an image of ancient Micronesian mariners who used natural signs rooted in Place - the stars, waves and bird - to guide their canoes across thousands of miles (Thomas, 1987). These sailors are analogous to participants navigation of the fluid and pluralistic sea of emergent identity studies. The participants continually explore the idea that we all have Hidden Treasures inside of us that can be revealed through identity work. Participants individual theories or Hidden Treasures became visible and unearthed multiple subjectivities as they explore how reuse materials can enrich equity-based identity studies. 


\section{Narrative session one summary.}

I interpreted the survival stage of Reggio formation through the archetype of the forager, then I interpreted the second and third consolidation and renewal stages of Remida formation through the archetype of the global homesteader. Finally, I interpret the fourth maturity stage of professional formation through the archetype of the navigator. Like a navigator who directs the route or course of a ship by using instruments or maps, here a strong identification with Reggio Emilia and Remida approach lead to the formation of a more sensitively attuned internal compass that steers Ann, Maggie, Joni, Julienne, Clara, and Miranda in their experiences with reuse materials and identity studies.

Now that we have looked at three metaphorical archetypes that emerged from narrative session one data interpretation and discussion, I turn to narrative session two interviews that followed about one month later.

\section{Session Two Dyadic Interviews}

In this section, I present data generated during the second dyadic interviews. Prior to the second session, I invited each of the participants to participate in an online scheduling tool: Doodle Poll to schedule their paired interviews and asked them to bring relevant artifacts to the discussion, such as Remida-inspired classroom documentation, curricula, photographs and/or videos as another way to share their experience and perspectives about socially constructing identities with children using reuse materials. The emailed message explicitly stated that the documentation was also intended to initiate relevant and genuine conversations about specific issues from their past or current 
work. Due to scheduling conflicts, the two CDC directors were unable to participate in the second narrative sessions.

However, the studio and classroom teacher dyadic pairs met on weekday and weekend afternoons throughout the winter and spring in a Remida-inspired room at the IRPP center to share their Remida-inspired identity studies documentation artifacts with each other. In most Reggio-inspired early childhood programs, educators document their daily experiences with the children through systematic observation and reflection to make sense of what they see and experience. Our process seemed familiar to this Reggioinspired documentation practice.

An ice-breaker hands on activity came before questions were posed to guide the third dyadic semi-structured interview. Here, the second session participants began with impermanent partner representations wherein they were asked to select objects to represent each other from a wide selection of a menagerie of colorful and provocative discards and natural materials. Two written prompts (a) represent each other using materials from the center and (b) share what it was like to represent your partner with reuse materials, were placed on the table during the second session with felt oval materials in different skin tone shades (Figure 5). 


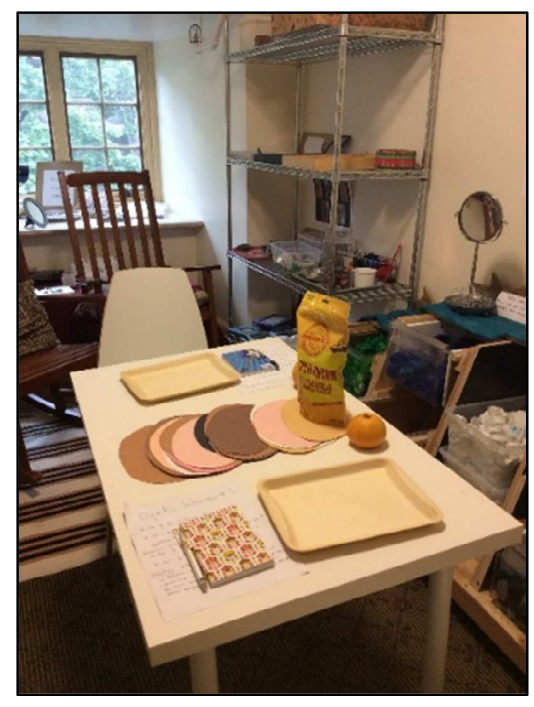

Figure 5. Session two dyadic interview.

\section{Studio teacher dyad: Ann and Maggie's session.}

Studio teacher Ann began sharing what it was like to represent Maggie with reuse materials and said, "When I look at you, I see that you always smile, and I see your teeth. Yours aren't craggily like these rocks but it is something that I noticed about you." Then she reflected on how the process of representing Maggie didn't cause her anxiety because they have known each other long enough. The pair laughed, and Ann added, "It is a fun process. We ask children to do this stuff all the time. It is good for us to try materials and to feel how that feels." The studio teachers agreed in unison.

Again, the studio teachers articulated their experience of broadening the scope of identity studies from physical to relational. Ann described how she enjoyed the excitement of wondering what materials she is going to find and the hunt of finding the pieces. At the same time, she described feeling, "that partial anxiety—will the person say, "Oh this looks fine," but be offended by it and think "Is that what I really look like?" 
Even though we clearly aren't representing our looks!' Studio teacher Maggie agreed and reflected on their discussion during the last dyadic session about the beauty of loose parts (Daly \& Beloglovsky, 2014). She reemphasized how the beauty of reuse material is that it takes away that very literal, "is this what I look like?" Maggie paused and then continued, "No, clearly you do not have glasses that look like bike gears and a bread tab for a nose, you know what I mean? It takes away some of that pressure." Ann concluded that the impermanent reuse portraits are fun (Figure 6), and the children at preschool like to do them often.

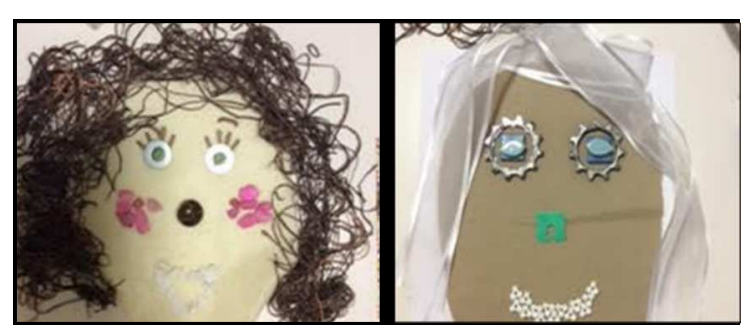

Figure 6. Ann's representation of Maggie (left) and Maggie's representation of Ann (right).

\section{Classroom teacher dyad: Miranda and Clara's session.}

The classroom teachers dialogue during the partner representation activity (Figure 7) continued to illuminate layers of broadening the scope of identity studies from physical to relational.

Miranda: It is interesting to represent someone you don't know very well. I remember I was looking for something to represent your hair and I was trying to find something different from what you used to represent yourself last time. Clara: It's perfect! [Laughing]. 
Miranda: It is so beautiful and lush. [Laughter] Did you cut it?

Clara: No, I was riding my bike out in the rain and I hadn't washed my hair. Miranda: I also picked something to represent your earrings because I remember you saying those were important to you.

Clara: Oh yeah!

Miranda: But they are not shiny! I just like the pokey balls! [Laughter] And then I put a little heart down here because the times that I have met you, you have always been so friendly.

Clara: Oh, that means a lot!

Miranda: I wanted that to be represented.

Clara: I like how my representation of you looks so cute! [Laughing] Miranda: Thanks, I like how simple it is.

Miranda began by saying, "It is interesting to represent someone you don't know very well. I was looking for something to represent your hair and I was trying to find something different from what you used to represent yourself last time." She told Clara that she picked materials to represent earrings because she remembered Clara saying earrings were important to her when she represented herself.

Then Miranda noted, "I put a little heart down here because the times that I have met you, you have always been so friendly. I wanted that to be represented." Clara gasped and cried out, "That is so kind!" She thanked Miranda and said that meant a lot to her. The representation Miranda made of Clara became less concrete or physical and 
more relational and symbolic of a characteristic of big heartedness or friendliness that Miranda had identified in Clara during their participation in this research study.

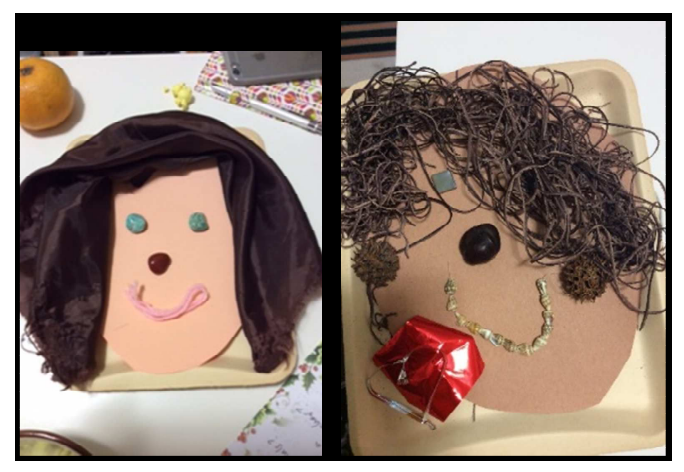

Figure 7. Clara's reuse portrait of Miranda (left) and Miranda's reuse portrait of Clara (right).

After the hands-on prompt to make impermanent representations of each other with reuse materials, questions were posed to the dyadic pairs to guide the third dyadic semi-structured interviews.

\section{The re-documenter.}

In the second session of dyadic interview, the was an important prompt, "What does documentation mean to you? Please share your documentation of reuse and identity studies," that permeated the data in a different way. The prompt was designed to make participants experiences with reuse materials in Reggio inspired identity studies visible. Throughout this experience, the participants shared various aspects of their identities through the documentation as they employed multiple metaphors and frameworks to describe what documentation meant to them. Classroom teacher Miranda identifying with documentation and shared, "I can't imagine working with children without documenting it. Documentation has become a part of who I am as a teacher and a part of 
the culture I am in." This distinctly reminded me of Clara's session one identity study, when she said,

We are sending a message through these materials because they are screaming possibilities that invent transformation. That is how I find myself-_Laughing] to transform and to create, that is how I find myself as a Remida-inspired educator. Similarly, to Miranda identifying with documentation, as a teacher Clara identifies with Remida materials, as having the same affordance inside herself of transformation and creation.

Interestingly, the participants varied widely in their approaches to sharing documentation. Studio teacher Ann used a digital camera to show her identity studies to Maggie. Studio teacher Maggie accidentally left her documentation in her car, so she shared her identity studies with Ann narratively. Classroom teacher Clara invited Miranda to her classroom at the Laboratory CDC (where the IRPP is located) to share her reuse identity study documentation panel. Classroom teacher Miranda brought physical documentation from her classroom to share with Clara, in the Inventing Remida room where the interviews took place. After the interviews, I collected digital copies of the Remida-inspired documentation artifacts that the studio and classroom teacher participants shared. These artifacts are re-documented below and interpreted in the sections that follow.

\section{Display of re-documented artifacts.}

The following five documentation narratives illuminate ways in which the Remida-inspired participants socially construct documentation to make social and 
ecological identities visible in their studio and classrooms with children ages 1 to 5 . Each of the five stories has a name: Looking Through; Grassy; Fantasy Lands; Cardboard People; Little Wooden Dolls; and Hidden Treasures.

First, Maggie narrated her documentation of Remida-inspired identity studies with her dyadic pair, Ann. To begin, she asserted that social constructivist ECE is identity studies,

I struggled with the lens of saying my documentation was an identity project; especially with infants and toddlers! I don't say 'Let's sit down and draw a portrait or create a portrait of yourself." Maybe I would at the end of the year once they have all turned two years old. It's not how we think about it with such young people. The first documentation that I identified was a journal from when I was in the studio that resonated with me as having identity contexts with reuse materials.

Maggie later shared with me digital versions of the documentation artifacts via email (see Figures 8a-d and 9a-b). These artifacts illustrate her cultivation of theoretical materials both in her studio and in the creative reuse cultural center at the corporate CDC where she works. 


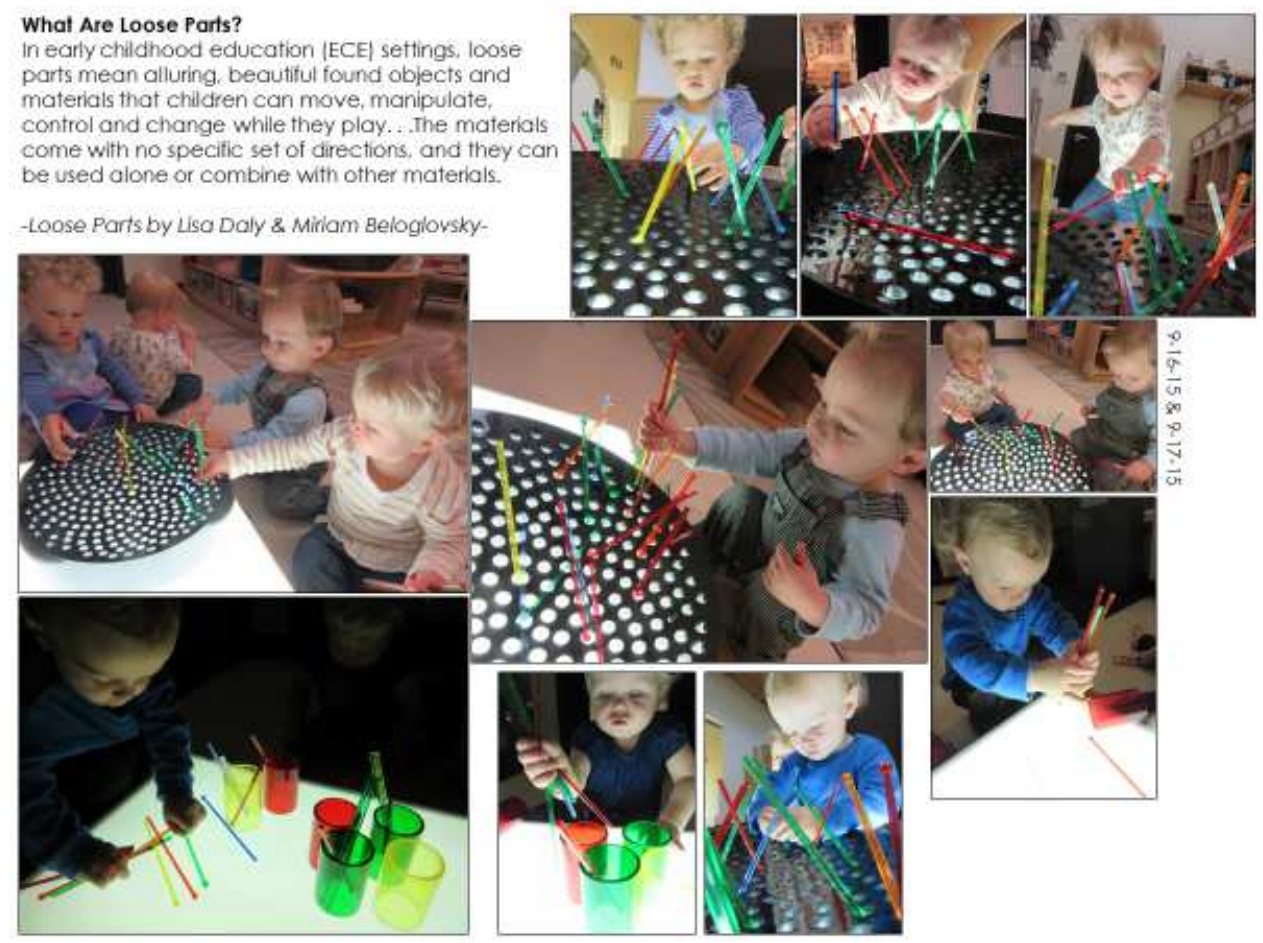

Figure 8a. Maggie's “Looking Through” documentation Part a.

In response to the fourth narrative inquiry prompt, Maggie expressed to Ann how she wanted to share her research of identity studies with infants and toddlers; a story called Looking Through. She read from her documentation journal the ways that very young children immediately interact with reuse material(s). Her journal entry read, "What that says about identity: When you are literally changing the way that you see the world and experimenting with that.” The documentation artifacts below (Figure 8b-d) illustrate collaborative inquiry with reuse materials as a way to encourage children to see through multiple perspectives. 
Discovering Possibilities in Our Studio:

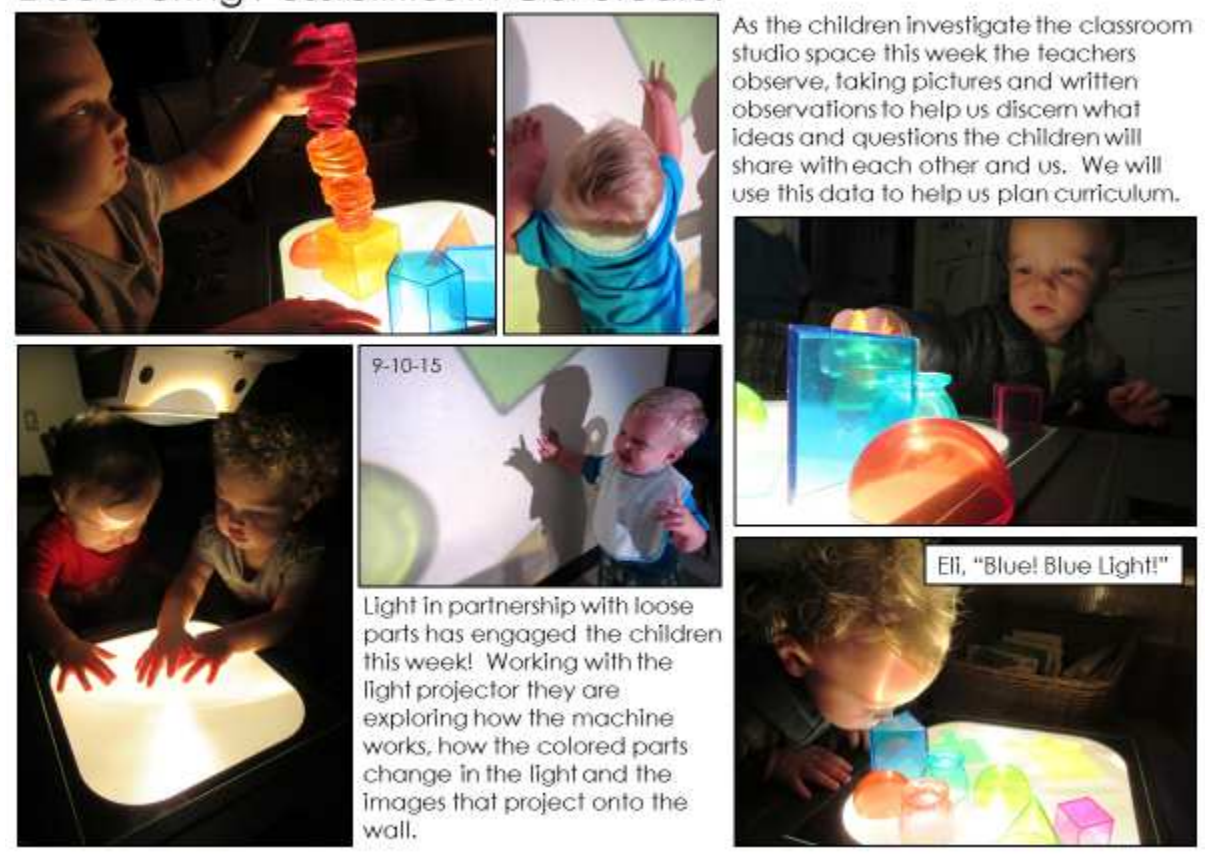

Figure 8b. Maggie's “Discovering Possibilities in Our Studio” documentation Part b.

Maggie recounted how she and her co-teacher were reflecting on the

documentation and wondering, "What if you give the children mirrors and you partner

that? They are looking outward and seeing things changed, but what would happen if we incorporated mirrors with that and they were looking at themselves changed?" 


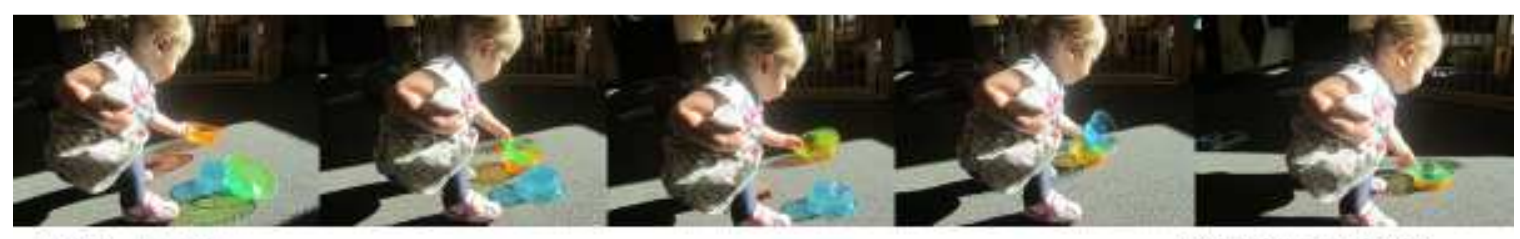
Apel $29^{\text {th }}-$ Mar 3rd
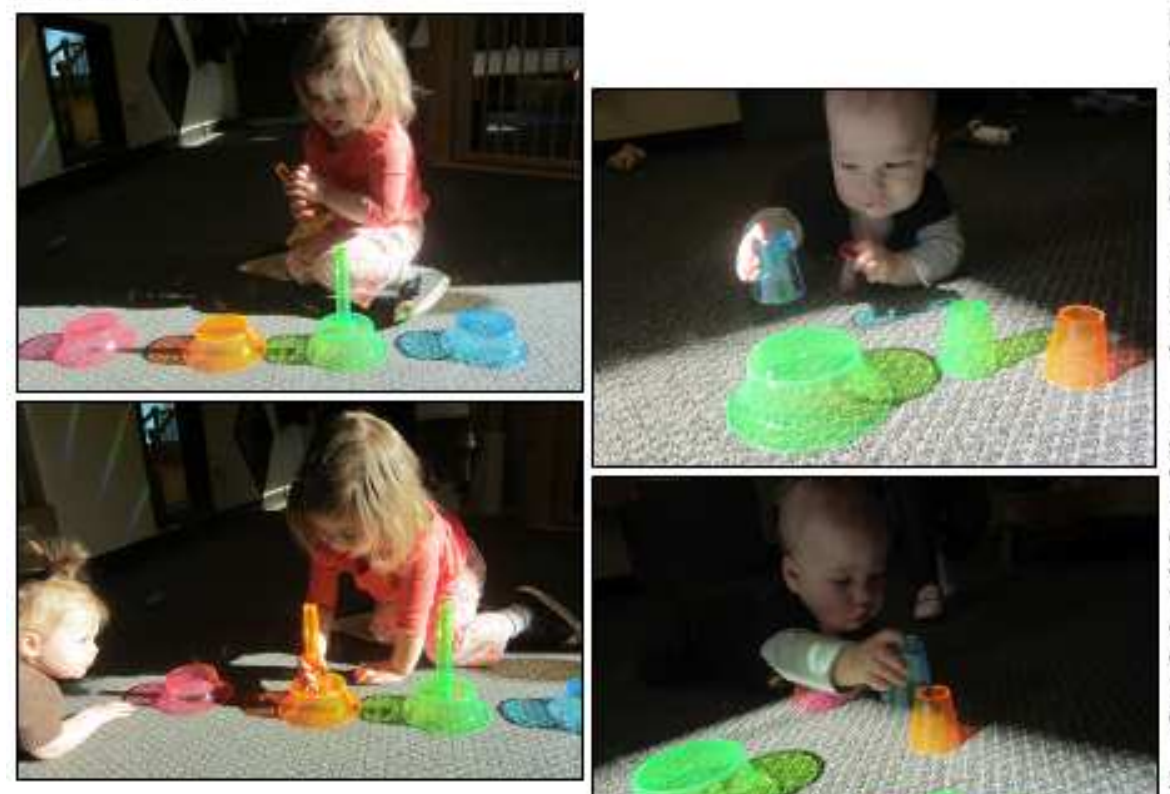

Using open-ended materials in the classroom we see how children at different developmental levels approoch the same material in unique ways. making the learning experience their own.

Lyla approaches a set of bowls. She begins to nest the materials together. one by one.

Elle approaches the some set of bowls. She walks away and retrieves the colcred test tubes offered on the light table. She returns and matches the fubes to bowls of coresponding colors. Elle explains,

"Green.... Orange. I need a blue."

Silas uses the same materials to experiment with creating sound.

Figure 8c. Maggie's “Open-ended Materials” documentation Part c.

Maggie highlights the found objects and how infant children are attracted to looking through translucent materials. She shares, "We use a lot of discard laminate paper for the baby rooms because they crinkle, and they will hold it tight against their face and look around." Maggie concluded, "Children begin identity studies in the toddler year, in my experience. First, they are identifying body parts on themselves and then on family members." 


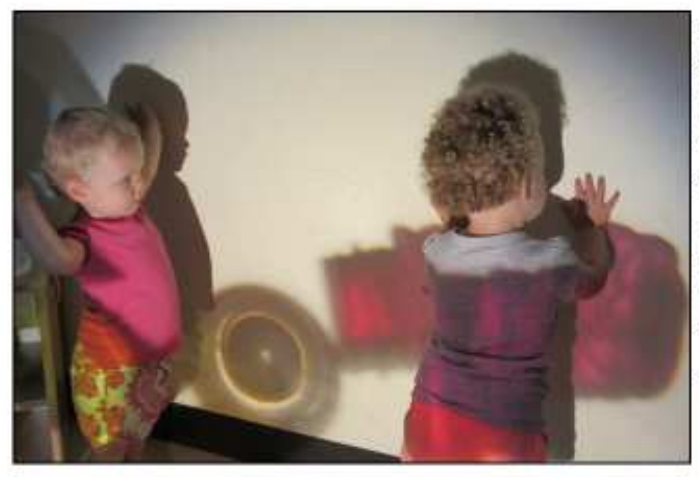

Eva becomes still, watching Morgan as she plays in the light. As Morgan steps back Eva moves closer and places both hands on Morgan's shadow. "Gan!" Eva shouts, Identifying the shadow as belonging to Morgan.

Moment later Morgan walks away and Eva stands alone, closely watching her own shadow in motion as she moves side to side.
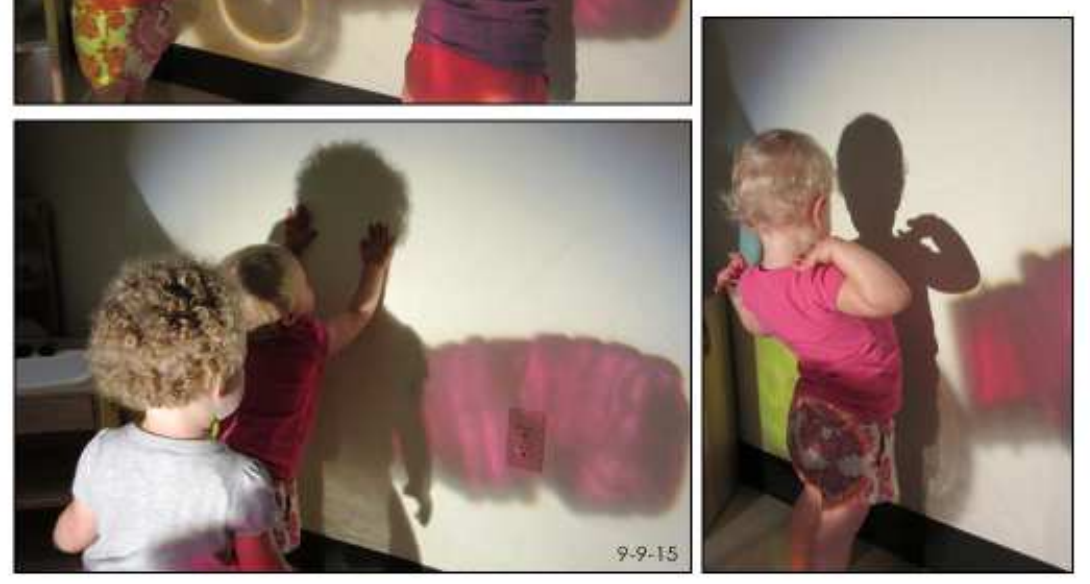

Figure 8d. Maggie's "Light and Loose Parts" documentation Part d.

Constructions of inquiry into light studies are scaffolded in the infant/toddler

years as children investigate natural light, light tables and overhead projector technology. Maggie's documentation is one example of collaborative inquiry; a blending of different perspectives, questions and hypotheses. It was formed by the voices of the educators and by the voices - both literal and metaphorical of the children and the alternative materials used.

Studio teacher Maggie also shared the next story, which was created with children two and three years of age. The story is called Grassy. In her words, "One of the interesting things that made me identify this as an identity project was that the characteristics ascribed to Grassy almost always mirrored characteristics of the children themselves." She explained her perceptions by narrating, 
The children had created an invisible creature named Grassy in their classroom, which happened spontaneously so there was no context of it's a tiger or a human or anything like that. The children who were making "Grassy" were 2 [years old] and part of the work they were doing in the studio at this point was creating individual art representations. Grassy was the name of the invisible friend they all shared, and all saw so differently in their minds. We were using reuse materials and black paper.

What made me identify this as an identity project was that the characteristics ascribed to Grassy almost always mirrored characteristics of the children themselves. Whether it was an attribute like, "Grassy has two legs," or "Grassy has green eyes" said by someone who has these beautiful green eyes staring back at you or "He goes super-fast" by a child who is a runner, these identities that were important to the children, that they had heard about themselves or identified in themselves.

The digital documentation artifacts Maggie provided me with me later via email (Figures $8 \mathrm{a}-\mathrm{d}$ and $9 \mathrm{a}-\mathrm{b}$ ) were part of her story about a toddler age child who chose discard materials from the corporate creative reuse cultural center to make an impermanent representation of "Grassy" and make her thinking visible. 


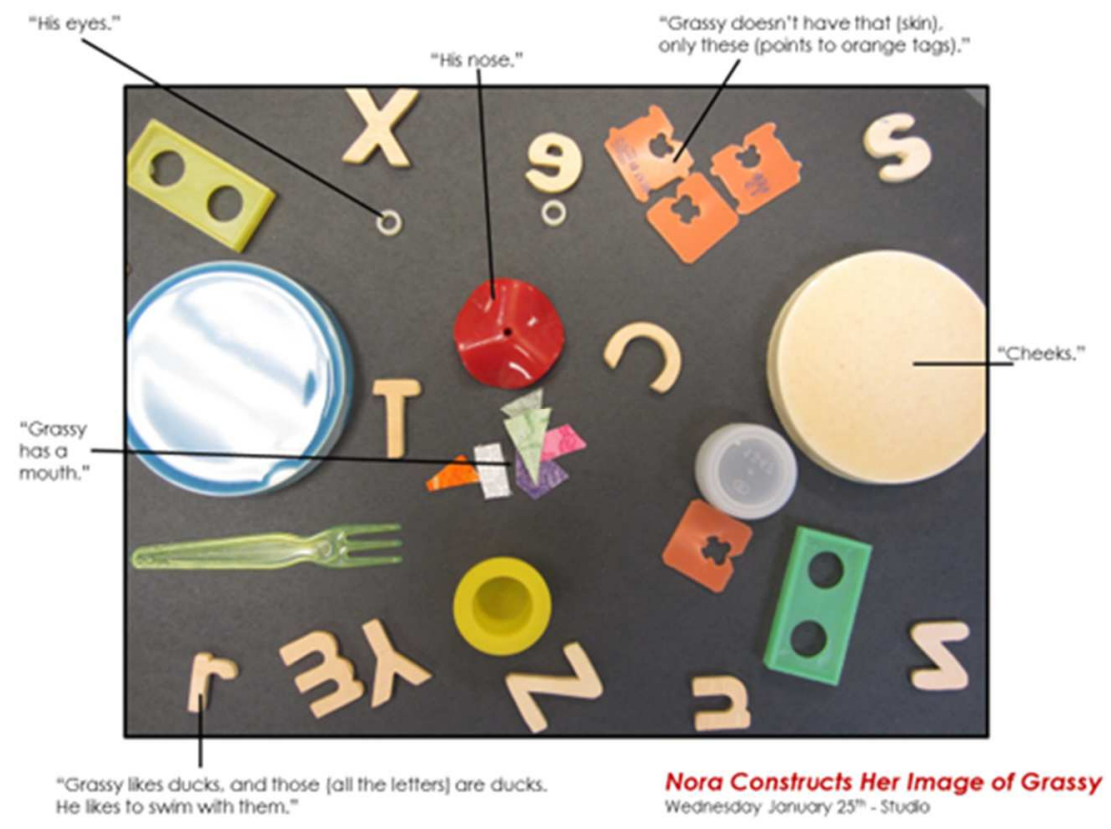

Figure 9a. Maggie’s “Nora Constructs her Image of Grassy” documentation.

Maggie explained how she could begin to see the formation of identity unfolding in the documentation. She stated, "Then some of the older children who were growing out of that a little bit would say things like, well, Grassy can have four legs, and start to dream up an identity of Grassy's own” (Figure 9a). 
In the studio today we began by reflecting on Nora's work from Wednesday when she created Grassy from re use materials. We also talked about some of the body parts Nora did not add to her representation:

Does Grassy have legs?

Tanio: Yes! He has 10 .

Aarush: He has 18, 5 in front and blegs in back. Nora: He hos just 5.

\section{Does Grassy have feel?} Noro: Nol

Tonia: Grassy has 5 tails.

Aarush: That's silly.

Tania: Grassy has little babies.

Nora: And the momma has little babies. Two bables. Tonio: Circle eyes.

Tanio: My Grassy jumps in puddles.

Nora: Grassy is a tiger. He likes to stomp in the mud.

Tania: I made Grassy. We're working on a tiger. My

tiger in so long.

Aarush: My Grassy eats ducks.

Does everyone see a different Grassy? Nora: Yes.

Does your Grassy look different than Tania's Grassy? Nora: Yes.
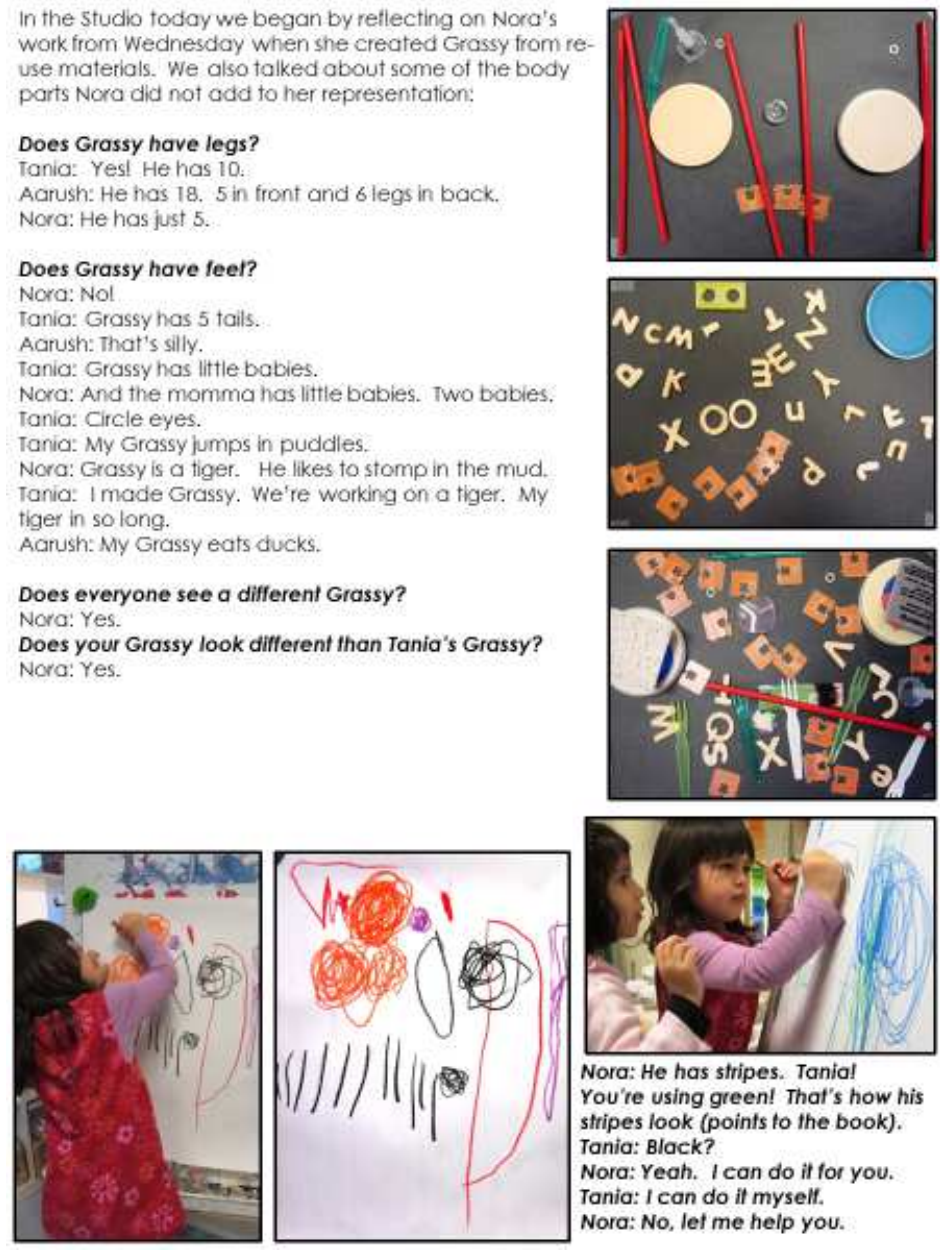

Figure 9b. Maggie's “Grassy” documentation Part b.

Maggie's documentation of identity studies with infant/toddler age children makes visible how in her perspective, everything a child does expresses their identities and supports them in learning about who they are in the world. Once Maggie was done sharing her documentation stories of Looking Through and Grassy, Ann began to share her documentation of identity studies with preschool age children in her wing. 
Next, Ann shares layers of her documentation as a studio teacher of a preschool wing. During the dyadic interview she narrated two stories for Maggie through her photo documentation.

I brought my camera. What we are working on right now is not specifically, with the intention of [fostering] identity, but I find that most things that you do wind up going back to that even if it is not as overt as "we are going to make a face" or "what skin color are you?" or "what color of hair do you want to have?" and yet their identity comes through in everything that they do in the studio and in the context of their family.

The digital photographs that follow were sent to me by Ann via email after the dyadic interview data was collected.

Ann narrated her story, Fantasy Lands, about how a small group of children came into her studio and make a house, and she asked, "What are other places people live in?" and the children said "Apartments!" so she asked if they wanted to make an apartment. Ann pointed out, "They have made a couple of houses in small groups (Figure 10a), you can see that they are very child made, and so one kind of leans over a little bit!" 


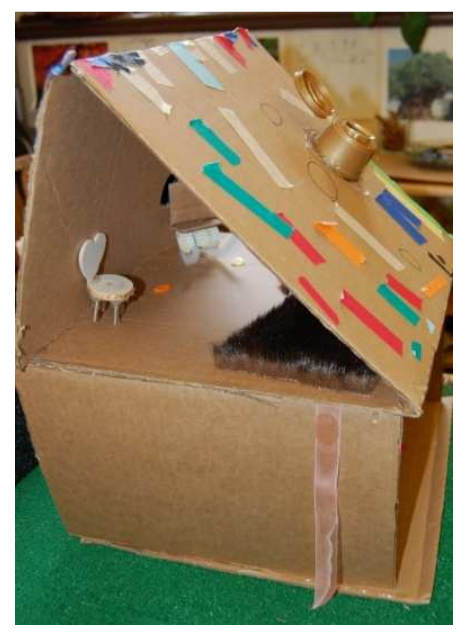

Figure 10a. Anne's "Fantasy Lands" documentation Part a.

Ann continued, "Then they started making furniture, everyone had a persona doll, they began to build things, we had made some cars, so we asked—what else do we want to build?" She reflected on how children building representations of their homes and vehicles is a part of identity studies.

During this dyadic session Ann and Maggie continued to look at her camera and documentation of a reuse car as an example of what her group made with recycled materials (Figure 10b). The photo also served as an example of the embeddedness of identity in children's Remida inspired studio work, "We were talking about how the car needs to have two seats because a child told us, "My dad sits here, and my mom sits here," and that is part of identity too." 


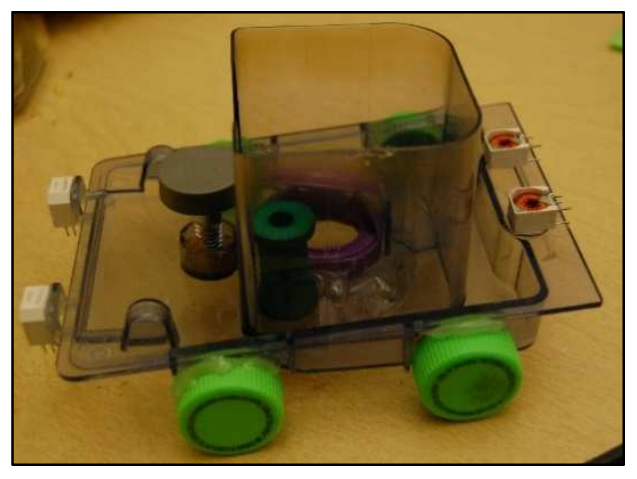

Figure 10b. Anne's "Reuse Car" documentation Part b.

Next, Ann began narrating her global homesteader experience of sowing the seeds of Early childhood education for sustainability (ECEFS) by asking the children in her reuse studio "Where do these materials come from, what's their purpose, why do we have them?" She noted how the children saw the discard materials as exciting materials to use in their constructions of "fantastical lands." Ann intentionally made connections, "Then we are adding in the loose parts. We have educated ourselves about materials and naming what these materials are: metal, wood, paper, glass, plastic" (Figure 10c).

Then during this same dyadic session, Ann shared pictures with Maggie documenting another story about a Remida inspired project with a combination of three classrooms. She explained that there were a little over sixty children coming through her studio, and how some were more involved than others, but everyone had opportunities to learn about recycling and to create using salvaged materials. She reiterated that across the preschool classrooms in her wing there was a collective interest in building houses and apartments and a little bit of a park — and how that started a "Big Idea" (Chaille, 2008) of making toys from salvage materials found in IRPP and the small-scale studio creative reuse cultural center. 


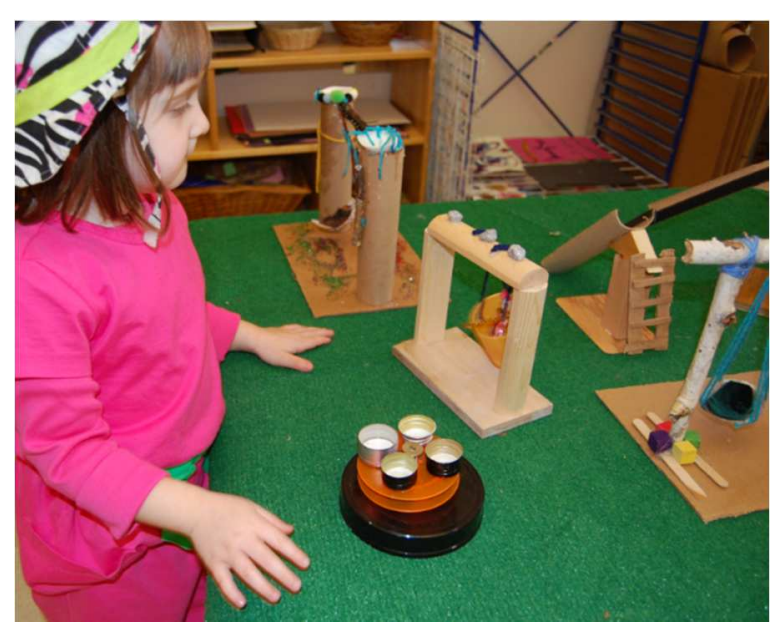

Figure 10c. Anne's “Making Toys from Salvage Materials” documentation Part c.

Ann continued sharing pictures from her camera with Maggie, "Some children were researching outer space, and they were talking about the idea of what kind of houses you can have in outer space, so we built those." The following picture (Figure 10d) is a documentation artifact of a house in outer space that the children created with Remida inspired materials. Ann emailed me this photograph after the interview data was collected.

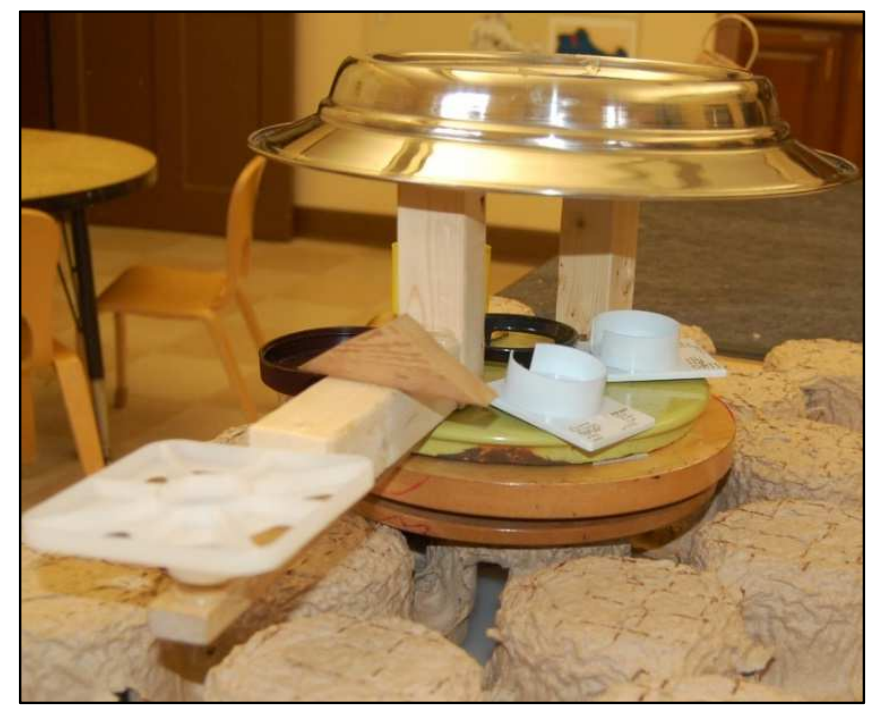


Figure 10d. Anne's "Discard House in Outer Space” documentation Part d.

Moreover, Ann described to Maggie how she and the children recently decided to pause that part of the project to focus on making plain little wooden dolls into identity characters. This documentation story she calls Little Wooden Dolls. She shared the photograph below (Figure 11a-b) and said:

Children have chosen what color they want to paint them the plain little wooden dolls; some have light or dark skin flesh tones, some are purple, some are yellow, or green-whatever they wanted, I left it up to them. Then they came back to the studio and started putting clothes on them. Many children made representations of themselves; they could make what they wanted. Some children made multiple dolls, so they have a little family, it really varies, and it was open like that. You started hearing "I want to make my mom, so I need a big one. I want a little one because I want to make myself," they were socially identifying their family.

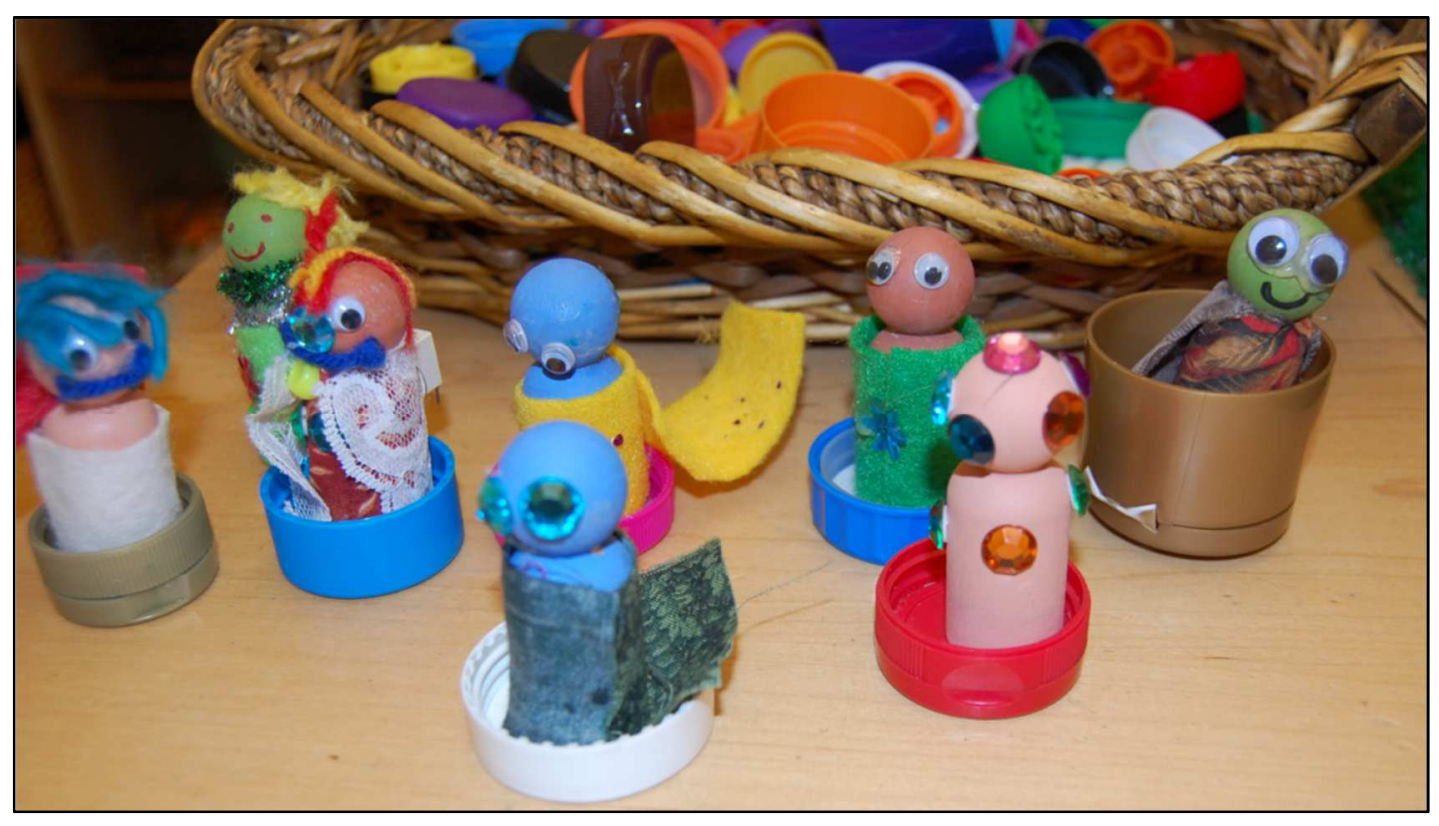


Figure 11a. Anne's "Little Wooden Dolls" documentation Part a.

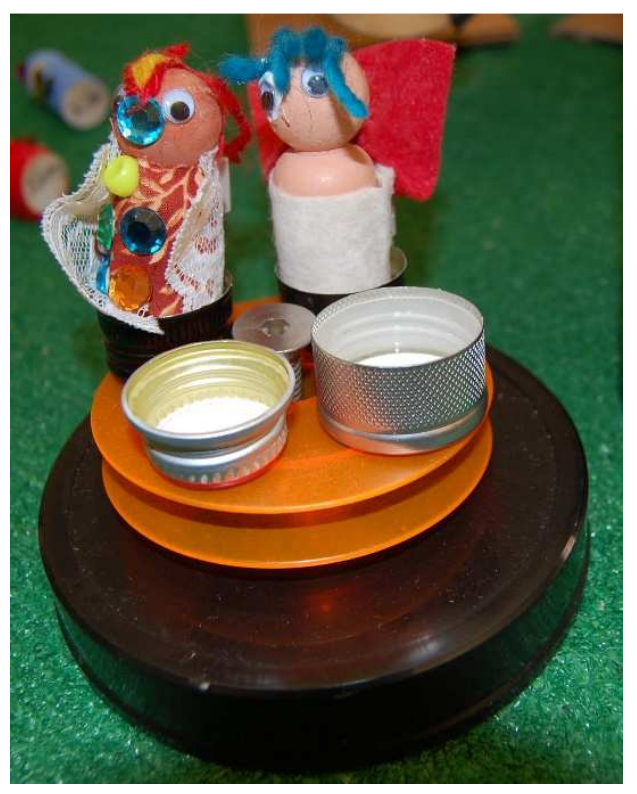

Figure 11b. Anne's "Reuse Identity Characters" documentation Part b.

Anne's documentation story illustrates how children's identities saturate so much of what they do in the studio and in the context of their family. Now I turn to Clara and Miranda's dyadic session. I begin with Clara's documentation of identity studies with preschool age children.

During Clara and Miranda's session two, Clara chose to share the layers of a Remida-inspired project called the Cardboard People (Figure 12a-g). Clara asked Miranda to hold the audio recorder while they walked downstairs to the second floor of the laboratory CDC to look at her 3- to 5-year-old classroom documentation panel. 


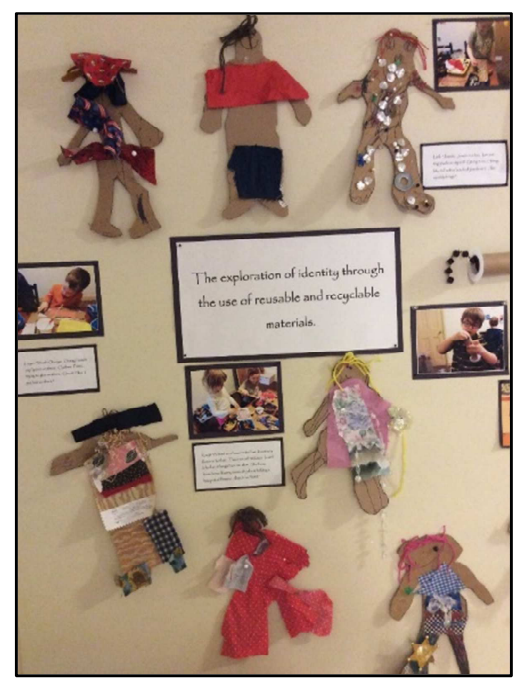

Figure 12a. Clara's "Cardboard People" documentation Part a.

Clara narrated, "At first we presented children with the possibility of exploring features of their faces, and then investigate how they are similar and at the same time different from each other." She continued, "During meeting time we read identity related books that presented the children with ideas of the many different ways we look, exploring not only our features but also our feelings and the ways we relate to each other." Clara explained how as teachers the goal was to zoom in on the children's physical features and discuss how we all look different and the ways in which we are all unique.

Clara continued, "The next step was to start slowly zooming out by tracing each other's bodies and identifying our likes and dislikes and aspects of ourselves that make us who we are" (Figure 12b). Clara described how the children talked about things they like to do, play, their favorite colors, favorite foods, special people in their lives and different languages that the children might speak. "All aspects of ourselves that make up our identities." Clara mapped the progress of the project for Miranda, and said, "It was very 
open ended. First, they traced each other bodies onto big pieces of paper, which we shrunk down and transferred onto discard cardboard and then cut them out." Inside the body tracings, each child drew a map of their individual and social identities.

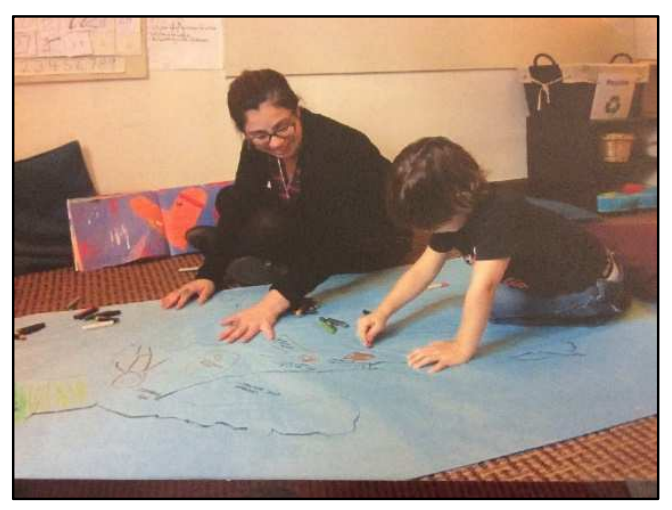

Figure 12b. Clara's "Body Tracings" documentation Part b.

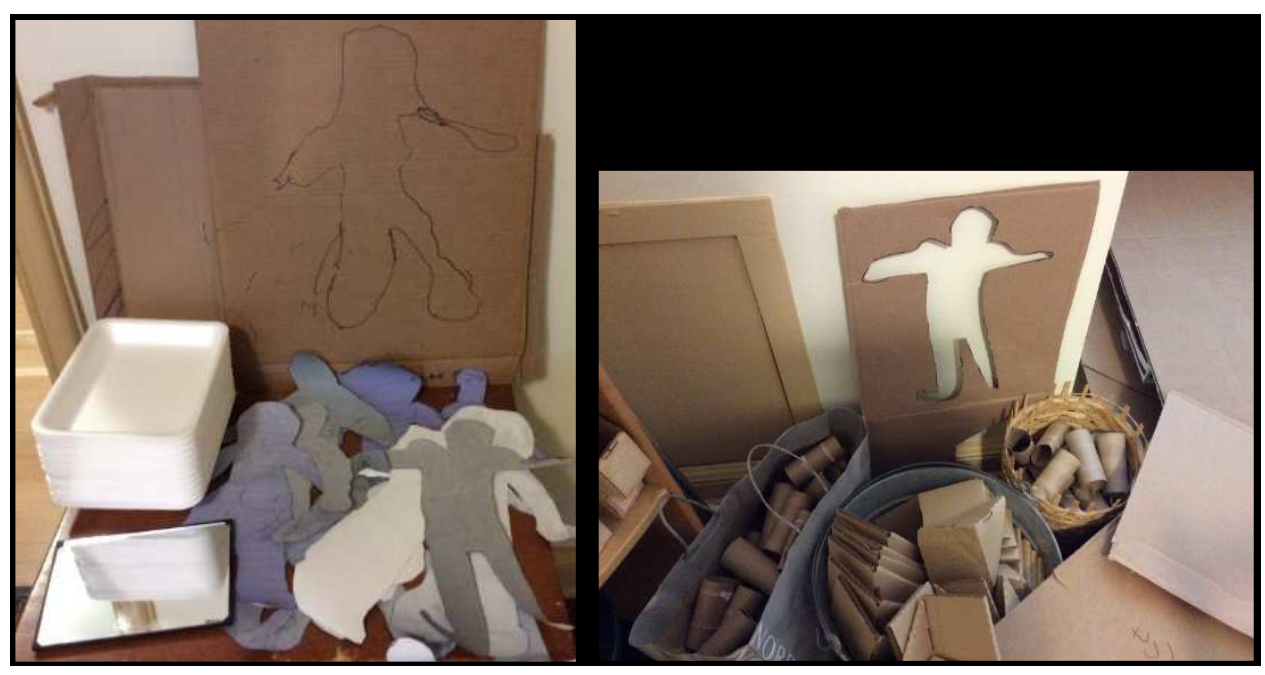

Figure 12c. Clara's "Languages of Fiber" documentation Part c.

Clara continued, "The class came here (Inventing Remida Project) and explored and collected materials that they wanted to use to represent themselves on the cardboard" (Figure 12c and Figure 12d). 


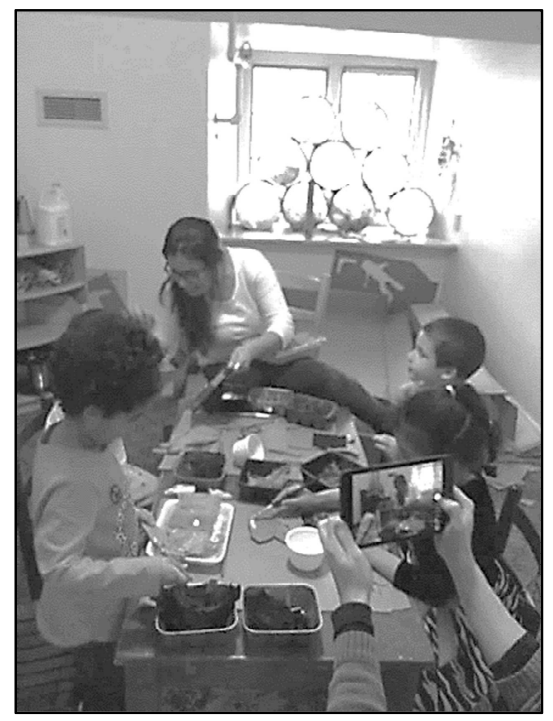

Figure 12d. Clara's "Reuse Atelier" documentation Part d.

At another stage in the project, Clara described how paper representations of the children along with reuse materials and an overhead projector were introduced in the IRPP workshop (Figure 12e). The provocation resulted in what Clara described as,

Some fascinating explorations of patterning, shapes, shadow, light and impermanent self-representations. Seeing the children playing together with their own shadows, smaller paper presentations of themselves and the shadows of those tracings made us see the way that children were creating and encountering multiple representations of themselves. 


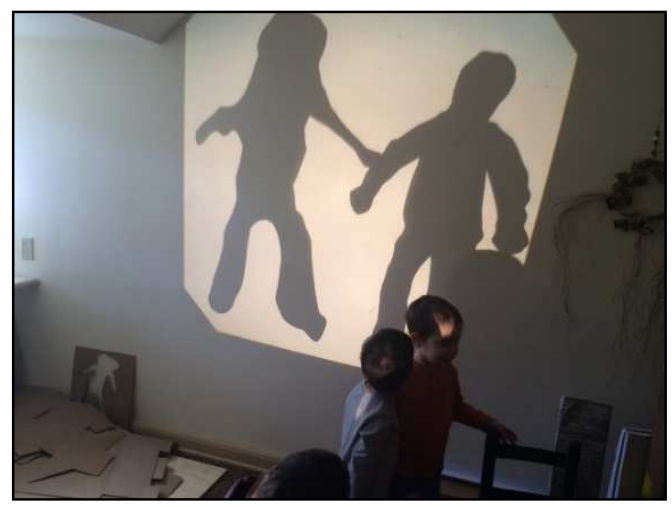

Figure 12e. Clara's "Multiple Representations of Selves" documentation Part e.

Clara chronicled how the Remida-inspired materials were introduced, and then her and her co-teacher proposed to the children, "How do you want to decorate your persona doll? - meaning yourself." She clarified how by inviting the children to each create their own persona doll meant an outward representation of themselves (Figure 12f).

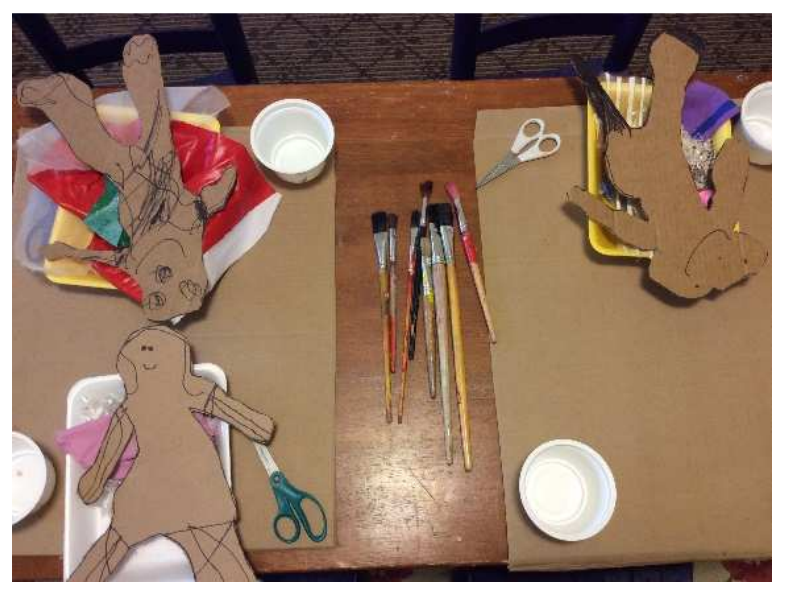

Figure 12f. Clara's "reuse persona dolls" documentation Part f.

Each child had their own approach to the ongoing invitations to create their own representations of self. Clara chose one child's narrative to highlight as she shares, 
Dima is a very happy sociable child. During the project she took great care and engaged with great focus at every step. When initially presented with her cardboard person, Dima remarked on the bulge on one side of the body and said it was her baby. Dima is a big sister and the arrival of her baby brother was an important even for her and her family. Her reaction to the proposal to draw her features onto the cardboard person was to quickly draw dots for eyes and nose along with a large mouth. She then proceeded to carefully trace the outline of the person onto the piece of cardboard beneath.

She noted how when asked to choose textiles that she would like to use to clothe her carboard person, Dima was quick to pick out a variety of fabrics. Clara continued, Dima spent a lot of time carefully adding detail when she attached fabrics to her person. She carefully and independently cut pieces of fabric to the desired sizes. She glued on the fabrics to make clothes along with other materials she was intrigued by including metal washers and small plastic beads. She also used a marker to add a patch of green color to one of her person's legs. When it came time for the children to add hair to their figures, I was intrigued by how Dima picked out pink hair for her figure. In an earlier conversation, Dima noted that her own hair was black but once she saw the pink yarn, she was set on using it for her cardboard person.

Clara described how she and the other teachers wondered what Dima's intention was with this choice? Was it connected with her developing gender identity? Or an aesthetic preference for pink? Then she stated, 
I have also noticed that Dima is drawn to hair that is dyed from colors in it. She often comments on our student teacher's hair color or asks me to see my purple hair. Could the choice for her cardboard person have emerged from a fascination with people who have brightly colored hair? During this experience Dima continued to seek out and add layers of detail to her carboard person including the addition of more beads. She formed a string of them into a spiral on her person's stomach and added a large gold star.

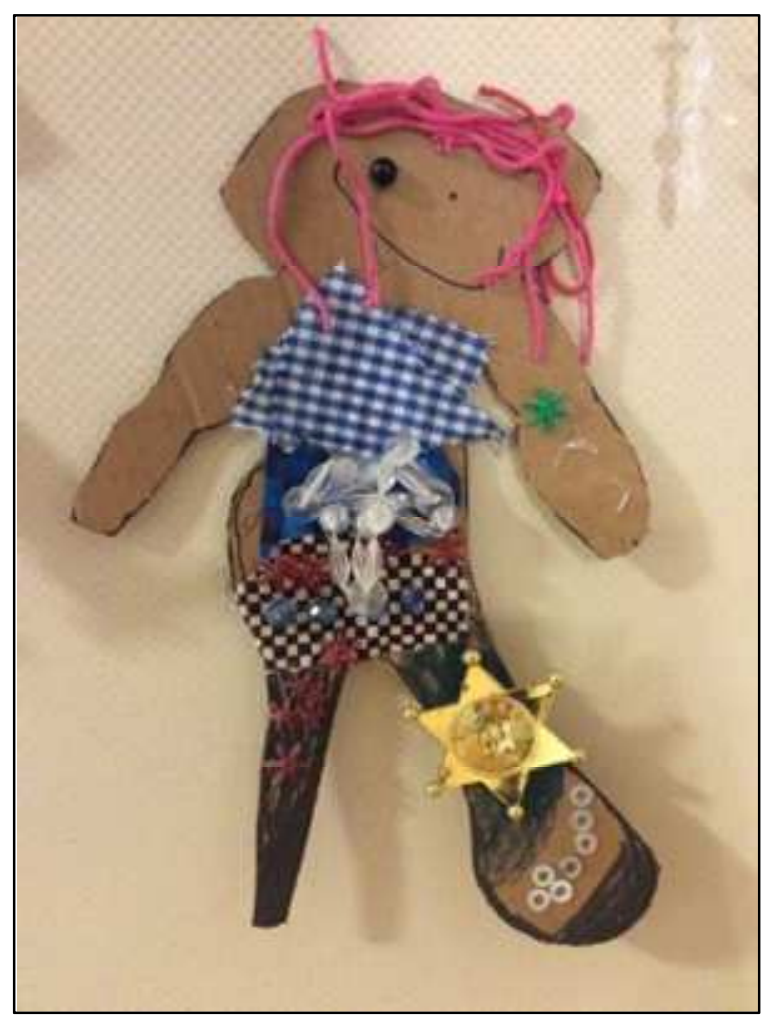

Figure 12g. Clara's "Girl with the Purple Hair" documentation Part g.

Clara remarked that it was interesting to observe how children chose reuse materials to represent themselves pluralistically, "They used different colors to represent their hair and to represent their thinking. If I could be this way, I would be this way-in 
my mind this is what I look like; this is who I am" (Figure 12g). Clara's documentation story makes visible her approach to identity studies as democratic and anti-bias work. Next, I turn to Miranda's documentation of identity studies with preschool age children.

Lastly, classroom teacher Miranda shared her Remida-inspired documentation story called, Hidden Treasures, with her dyadic partner Clara. She shared the inspiration for her identity work coming from the shared reading in her learning community of the works of research professor Brene Brown (2012) and memoirist Elizabeth Gilbert (2015). She brought printouts of her documentation which she shared with Clara in the identity atelier during session two. Then she told the story of the creative reuse project, "This year we took a next step, inspired by Gilbert's book Big Magic and the idea that we all have hidden treasures inside of us and that we could reveal those through identity work." She continued, "It's that intimate idea that you are someone who deserves to be heard and valued and understood." Miranda put this statement into context for Clara by explaining how, after reading Brene Brown at work and listening to Brown's TED Talk on courageous vulnerability a few years ago, she and her co-teacher started thinking about the idea that all children, all people, want someone to listen to them, to get to know them. Miranda explained how she wanted to bring to light the uniqueness of each child through their relationships, "We adopted that idea in our classroom in a way that we could make visible." The identity project was long term, "Teachers and children as a group had a two-year span of time when we thought about: Who are you? Who am I? Who are we together? Then we dissected these ideas a little further and thought about each child and teacher." 
Miranda described the "next layer" as after having documented the identity study with the same children over time; Miranda and her co-teacher were able to revisit old documentation and reflect on the new.

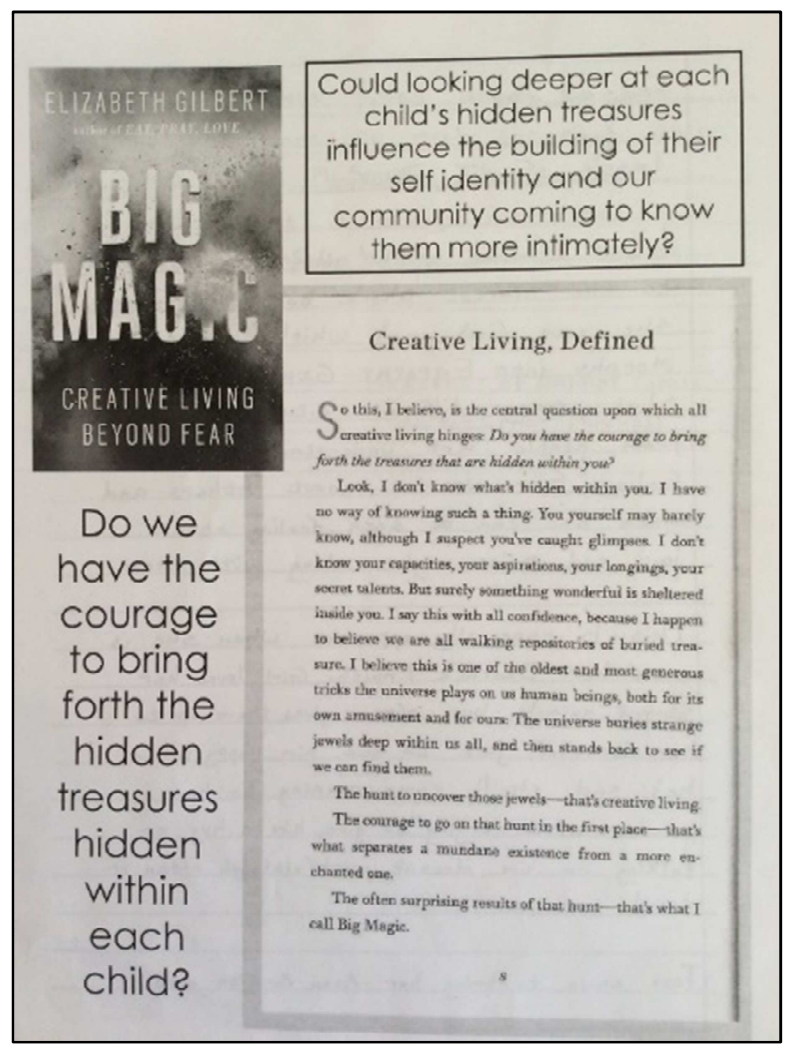

Figure 13a. Miranda’s "Hidden Treasures” documentation Part a.

Miranda described to Clara how she sent a paper notebook home with families and invited them to write an entry about their child's "hidden treasures" (Figure 13a) and she exclaimed, "What the parents have written has been so powerful! What they value in their child, what this question helped them to think about is just cool!" The notebook also suggested that children could bring in an item or idea that represented something that they want to share with the class. Miranda explained that unlike any other show and tell 
experience, the children and families sensed there was something deeper with a different intention provoked here. Children brought discarded materials from home that had stories hidden within them, such as buttons from a grandmother, unique paper, colored glass found on walks, broken tiles from a home remodel, textured fabric of old blankets, scraps of leather and metals from a dad's shop, interesting containers, and costume jewelry from their homes.

In broadening the scope of the identity study from physical to relational, Miranda detailed how she and her co-teacher framed the project experiences. She shared "We developed some questions that provoke their thinking. Then we did some drawing about it." She explained,

They would start out by sharing the journal entry in a meeting time with the whole community. We read it together and then we break up into small group, so a smaller group of children would sit together with this child named Jack and we would re-read the entry and think about what he brought.

Then the adult would ask the children, "What do you know about Jack? What do you love about him? They often talk about the word friend, so we ask why do you consider him your friend? What do you like to do together?"

Miranda then shared the following documentation of a protagonist named Tess in her classroom (Figure 13b). Here, a child was invited to make visible the individual and social dimensions of her identity through a self-portrait alongside images of her in relationship to others. 


\section{What are the treasures hidden within Tess?}

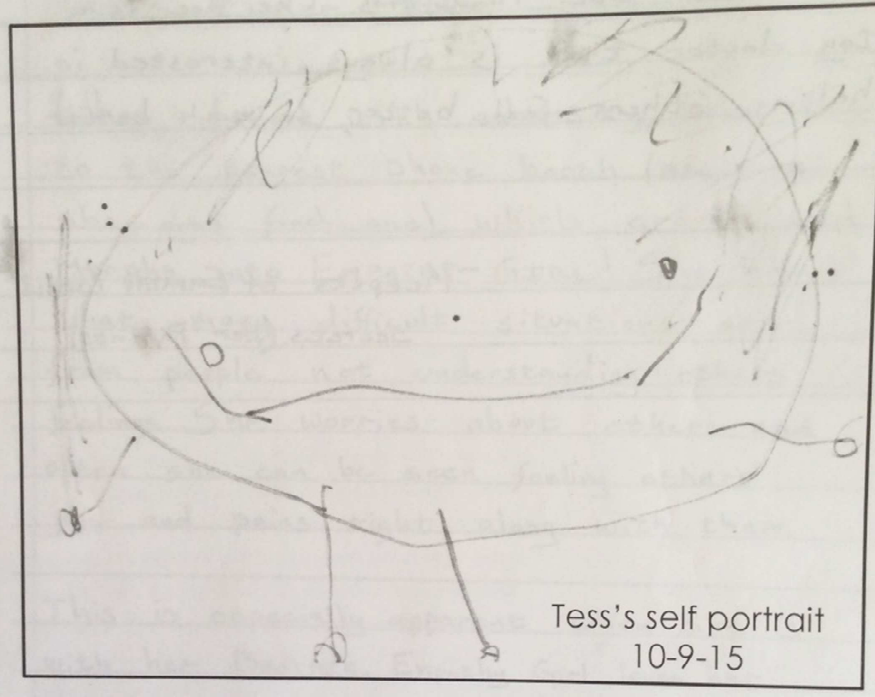

"The universe buries strange jewels deep within us all, and then stands back to see if we can find them."

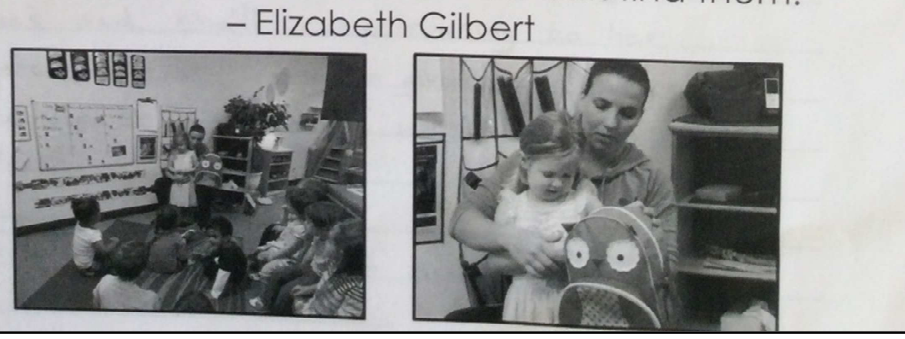

Figure 13b. Miranda's “Social Dimensions of Identity” documentation Part b.

Miranda continued,

We do it as it feels natural. The next week if we see that someone hasn't had a turn to share their journal or material that they want to share with the class, yet we will choose them. Sometimes a child will ask if they can be next. At a meeting time we will ask children if they have anything to share or if they have any questions for a child; "Do you have any questions for Jack or about his lovey-he brought his lovey." 
Then, Miranda shared another identity study artifact documenting a small group of children's reflections about the hidden treasures inside of their friend, Emmet (Figure $13 \mathrm{c})$.
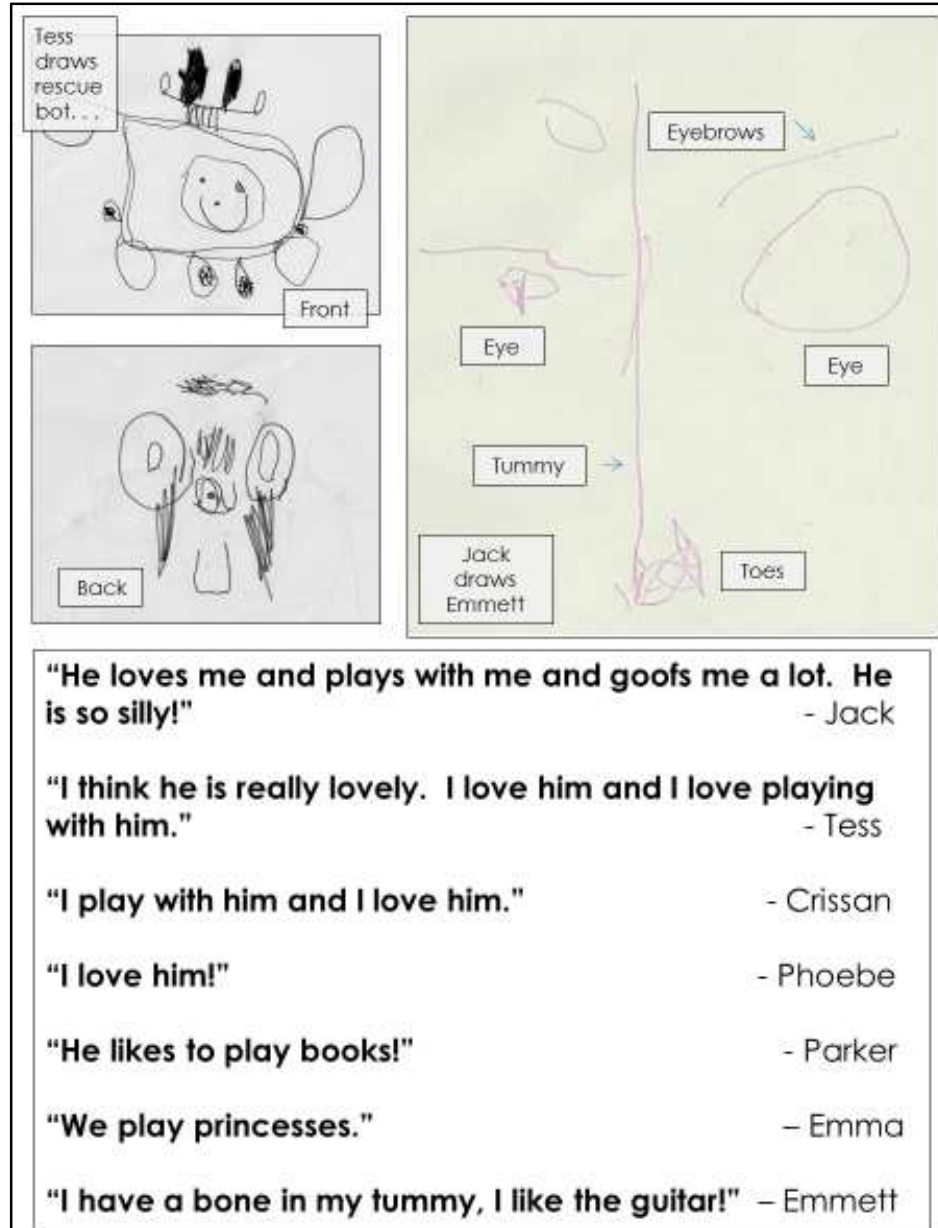

Figure 13c. Miranda's “Identity Study” documentation Part c.

Miranda concluded her story with how her learning community continues to conduct several small group hidden treasure sessions together. 
Data interpretations three: Growth pattern--The re-documenter.

Over time, I continued to reflect on the re-documented artifacts the participants shared in response to the fourth prompt: "What does documentation mean to you? Please share your documentation of reuse and identity studies." And over time, five professional growth patterns came into focus. Three of the growth patterns aligned with a mature stage Remida-inspired educator who approaches identity studies as a navigator: voyaging backwards from we to me; recovering a multiplicity of identities studies and; broadening the scope from physical to relational. And two of the professional growth patterns that emerged in the re-documentation align with the consolidation and renewal stages of a global homesteader: Sowing early childhood education for sustainability (ECEFS) and cultivating theoretical materials. In the following summary I will interpret these patterns as they emerged in the re-documentation, in greater detail.

\section{Interpreting 'Looking Through' through the lens of the Global Homesteader.}

Studio teacher Maggie's re-documented artifacts reveal a consolidation and renewal professional growth pattern: cultivating theoretical materials. Both of Maggie's documentation artifacts Looking Through and Grassy, record how materials were cultivated from family's homes as found objects, loose parts (Daly \& Beloglovsky, 2014) and "beautiful stuff" (Gandini \& Topal, 1999). The children were invited to engage the languages of reuse materials with natural and artificial light sources in her studio which triggered a series of hypotheses both verbal and non-verbal. These artifacts illustrate Maggie' cultivation of theoretical materials as a Remida-inspired "global homesteader" 
both in her studio and in the creative reuse cultural center at the corporate CDC where she works.

Looking Through makes visible Maggie's use of light to augment the language of reuse materials, and the image of the child as a co-protagonist in the stories Maggie tells about identity formation and how looking at the world from different perspectives and through reuse materials is an identity study. Maggie illustrates how she engages Remida inspired materials from her creative reuse cultural center with a complex investigation of light, reflection and brightness. To add to the investigation, Maggie turns on the light table and set up a series of colorful provocations including discarded translucent and opaque cups, bowls and straws. The children's curiosity guides them into new discoveries about color. The provocation can be interpreted metaphorically as an antibias proposal for how a different vantage point can change your perspective.

Multiple metaphors illuminate the narrative data I collected to pursue my main research question in Chapter 1, "What are early childhood educators' experiences with reuse materials in Reggio Emilia inspired identity studies?” In dyadic session two, Maggie re-documented journal observations of toddler age children picking up multiple discarded transparent bowls of different colors and looking through them. In her journal, she reflected, "What that says about identity— when you are literally changing the way that you see the world and experimenting with that." The Remida inspired documentation she shared makes visible how seeing through multiple perspectives signifies an identity study. This made me reflect on how young children looking through 
multiple discard lenses is analogous to interpreting identity studies through multimetaphorical meta-theoretical frameworks.

Interpreting 'Grassy' through the lens of the Navigator. Maggie's second redocumented artifact 'Grassy' reveals a mature stage professional growth pattern: voyaging backwards from we to me. Maggie's Remida-inspired documentation of learning made visible with infant and toddler age children entitled Grassy, highlights how identity is socially constructed and expressed through children's subjective feelings about themselves and about others. Children are social actors with personal agency as they construct their own image and meaning of grassy. This documentation demonstrates how over time, the children have an increasing awareness of "self as subject", (or "I") as evident in how they can say that they each have a different way of seeing "grassy" and not only as an extension of themselves.

Vygotsky (1978) once remarked, "Every function in the child's cultural development appears twice: first on the social level, and later, on the individual level; first, between people (inter-psychological) and then inside the child (intrapsychological)" (p. 57). For Reggio-inspired educators like Maggie, documenting identity studies are a social construction, shaping and being shaped by the school.

Interpreting 'Fantastical Lands' and 'Little Plain Wooden Dolls' through the lens of the Global Homesteader. Ann's re-documented artifacts 'Fantastical Lands' and 'Little Plain Wooden Dolls' reveal a consolidation and renewal professional growth pattern: Sowing the seeds of early childhood education for sustainability (ECEFS). Ann narrated her experience in her reuse studio of asking children, "Where do these materials 
come from, what's their purpose, why do we have them?" She noted how the children saw the discard materials as exciting materials to use in their constructions of fantastical lands. Ann intentionally connected their creative reuse and social identities to Reggioinspired culturally sustainable environments, "Then we are adding in the loose parts. We have educated ourselves about materials and naming what these materials are: metal, wood, paper, glass, plastic.” Sustainability education was sown by Ann, as she explored what the loose parts and reuse materials are made from and the possibilities for creatively reusing them with the children in her studio.

Further, Ann shared a vignette of how one child represented his family traveling through the fantastical lands as "space inhabitants." Ann described how he transformed three little plain wooden dolls into a space family with discarded materials, "Two aliens for the parents and a baby that was him. He has one eye, his mom has two eyes, and the dad has three eyes. Even through those, the children are showing you their identity." In this reuse identity study, the seeds of early childhood education for sustainability (ECEFS) were sown through proposing children express their family social identity with discarded materials. This included reuse as a language to express children's imagination of inhabiting physical universe beyond the earth's atmosphere, like the child who represented his family as "aliens."

The Literature Review in Chapter 2 records how as children construct their identities and perceptions about their self, these selves are shaped by local environment, values, and by their unique formation. The ecological consciousness scaffolded through creative reuse cultural education is divergent and seeds are sewn locally, globally, and 
even in children's consciousness of outer space or in their representations of "fantastical lands" made with Remida-inspired materials.

Fantastical Lands and Little Plain Wooden Dolls also highlight family identities as a source of social identity. As I noted earlier in chapter one, emerging literature on family identity in early childhood education shows how family is the most basic element of self-awareness for young children; it shapes and informs their sense of self and who they are in the world (Kissinger, 2017; Weiss, Caspe, \& Lopez, 2006). Woodhead (2006) highlights that identity has two distinct aspects, "That of the unique individual person and that of the shared social person" (p. 6). Ann's documentation echoes research on children's constructions of personal identity through relationships with others. Ann's observations about children's representations of family identity remind me of how social identity refers to the ways in which children feel they are (or would like to be) the same as others, typically through identification with family and/or peer culture.

Ann also shared her project work to emphasize how, in her view, children's social identity comes through in everything they do. Ann and Maggie both continued to emphasize how social constructivist early childhood education is identity studies. For example, Maggie reflected on children's representations of their homes and vehicles during her project entitled Fantastical Lands as an extension of identity studies. Through Maggie's Remida-inspired creative reuse, ecologically sustainable connections were made between children's social identities as members of family units as well as architects and inhabitants of built environments. As Vygostky (1978) stated, “Through others, we become ourselves.” (p. 38). Family systems theory (Bowen, 1978) suggests that 
individuals cannot be understood in isolation from one another, but rather as a part of their family, as the family is an emotional unit. Indeed, families are the first culture group in which children perceive membership (Carlo et al., 2010).

Creative reuse helped the children and Ann to think deeply about spatial, ecological and relational aspects of identity. Additionally, collaboration amongst educators, children, and families is a reoccurring theme in the early childhood education for sustainability literature as a means for children to experience the power of community to bring about social and environmental justice (Blaise, Hamm, \& Iorio, 2016). Ann's observations about family identity remind me of how social identity refers to the ways in which children feel they are (or would like to be) the same as others, typically through identification with family and/or peer culture.

Interpreting 'Cardboard People' through the lens of the Navigator. Clara's redocumented artifact "Cardboard People" reveals a mature stage professional growth pattern: recovering a multiplicity of identities studies. Re-documenting the project helped Clara to reflect through chronicling what took place and then reflecting on the process by looking at pictures, notes, and by going back through the story. She described to Miranda how she began looking with her co-teacher and IRPP coordinators at the next level of the identity study by asking "why is this important?" What bubbled up to the surface was the children's multiple and fluid representations of self through the reuse materials.

She continued to share how she, her co-teacher, and IRPP coordinators liked the idea of recycling literacy and identity studies and built on it to continue to discover and learn 
about creative connections. Clara physically revisited the documentation and the layers of the finished cultural production which was the cardboard people on display (Figure 12a) in front of her classroom on a documentation panel.

Clara's Remida-inspired identity study was guided by the enduring question that emanated from the IRPP project: How can we connect ourselves with reuse materials and see each other through reuse materials? The documentation she shared (Figure 12a) supports the problem posed in Chapter 2 that many developmental psychologists think preschool age children are incapable of expressing values around their social identity. Many developmental psychologists believe children are only able to express the following about their identity_-behavioral and dispositional qualities about themselves such as the color of their eyes, their age, their address, which hand they write with, their favorite color, and their favorite things (Bennet, 2004, 2011; Bennet \& Sanni, 2004).

Developmental psychology is the scientific study of the changes that occur in human beings over the course of their life. Previous findings in the field show that until the age of eight, young children's conceptions of social identities focus largely on behavioral and dispositional properties as opposed to belief-based aspects of identity (Bennet, 2004, 2011; Bennet \& Sanni, 2004). Clara's documentation makes visible a progression from behavioral and dispositional identification (hair color and favorite colors) to children's choices of materials to represent their belief-based aspects of identity such as nationality.

Clara expressed that this project went beyond simply creating self-representation dolls. "It went even further, and in the end, they were creating musical instruments to 
represent themselves, so it was amazing. It is a very beautiful and thoughtful and powerful way for them to represent themselves and explore this idea of self. This project took quite a while and they got deeper into it." Clara shared how they bridged the gap between the materials and Early childhood education for sustainability (ECEFS) through the introduction of upcycled fabrics to the project. Cardboard persona doll clothing design was accompanied by children's co-research with IRPP on the lifecycle of textile material; where textiles comes from, how they are made into clothing and their large presence in landfills after they are discarded.

Interpreting 'Hidden Treasures' through the lens of the Navigator. Miranda's re-documented artifact Hidden Treasures reveals a mature stage professional growth pattern: broadening the scope from physical to relational. Miranda's relational ideas bring us back to Warrin's (2010) study of the stories of pre-school children, which highlight personal aspects of identity emphasizing the educational importance of personal and social identity. Children establish their own identity through comparing themselves with other children and that children starting preschool seem to like school better and adjust if they have found friendship. Friendship is of value to children as they help each other understand the world in which they live.

These ideals are represented in Miranda's work with children and families and can be seen in the documentation artifacts she shared (Figure13a-c). As Currie, Kelly, and Pomeramtz (2007) note, membership in a group or exclusion from it, can greatly inform the construction of "who you are" in terms of identity, both in relation to one's own self-identity and how others see you. The children in Miranda's were encouraged to 
reflect on their own and each other's hidden treasures and unique characteristics as an evolution from the physical identity studies that inspired the project.

To summarize the main points made in this subsection, a re-story of the narrative data follows. The re-story combines six participants voices into one protagonist's voice. Once more, specific metaphors used by the participants are italicized in the re-story and it is written in single-spaced to evoke the spirit of the narrative voice. The purpose of this re-storying technique is to share the group of participants' individual experiences of redocumenting their creative reuse identity studies.

\section{A re-story: The Re-documenter.}

I can't imagine working with children without documenting. Documentation has become a part of who I am as a teacher and a part of the culture I am in. Documentation is the story of the life of the children and their center, and the story of studio and classroom teachers collaborating with the children; it's our story.

Documentation shows our commonalities as a community or the growth that we have had as a community and it brings to light each of our uniqueness. Documentation helps me to reflect through chronicling what just happened and then by looking at pictures, notes, and by going back through the story I begin looking at the next level by asking why is this important? There is something that bubbles up every time; you don't realize it when you are doing it, it may be the physicality of creating and what happens in the process of doing that?

Documentation reveals a story that is imbedded deeply within the play and wouldn't otherwise surface, it would just pass by. Videos and series of pictures can show what I missed; metaphorically speaking I might realize what I thought what was important here was the 'orange,' but what was important was the 'texture, or the smell.'

Documentation has a lot to do with progress, maybe it's the map to progress? Or the way you start that journey? When you bring a journal back to a child and say this is the work that you did, that changes their thinking too and moves them forward. When we describe the situation and the interaction between the children and the materials, it is like a dance. The next layer, when you've documented over time and sometimes over years with the same children, is looking back at old 
documentation and reflecting on the new because tells so much about who they are and who they have become in their journey of growth.

Now that we have considered what documentation means to the participants, from here I turn to the final narrative session three which was a focus group discussion and hands-on reuse provocation. The focus group-oriented activity culturally produced two re-storied personal dolls who demonstrate the possibilities of enriching early childhood anti-bias education through reuse materials. The reuse persona dolls epitomize the power of identifying with documentation and reuse.

\section{Session Three Focus Group Discussion}

I facilitated the third and final narrative session on a weekday summer afternoon in a natural cedarwood conference room with skylights at the IRPP center with two studio teachers, two classroom teachers and one director as the corporate CDC director Julienne was unable to attend. The participants were asked to break into two small groups from each CDC to collaborate on the reuse-oriented activity prompt: In your small group socially construct a reuse persona doll that represents a Remida-inspired educator. The dolls embody and are the product of the discussions between participants in response to the prompt.

Just as people naturally tell stories, humans as social beings have long been gathering together and discussing important issues in groups. Focus group researchers facilitate this naturally occurring performance and have refined it to make it a method of research (Morgan, 1998). One limitation of focus groups is that although participants are part of a group, the data analyses does not always consider that the opinions and ideas 
discussed are also products of the group. Instead, a sequence of the individual's contributions, rather than the part of the discussion between participants, are usually reported (Colucci, 2007). To take advantage of the social dynamic inherent to the experience of the being in a group, I proposed a reuse activity-oriented prompt to generate three-dimensional documentation in the form of two persona dolls (Whitney, 1999).

In keeping with a prominent Chicana approach to social science research, I chose narrative inquiry because it provides access to marginalized voices and allow for counternarratives. Anzaldua (2009) coined the term autobiographical theory or auto-historiateoria, which signifies a transformation of traditional Western autobiographical forms informed by reflective self-awareness in the service of social justice work. Auto-historia focuses on the personal life story but, as the auto-historian tells her own story, she simultaneously tells the life stories of others (Keating, 2009). My intention was for the persona dolls to carry the storied identities and to simultaneously tell the life stories of others.

On the conference table, two 12-inch-tall stuffed Muslin gender neutral craft dolls were available as well as loose parts (Daly \& Beloglovsky, 2014) collected and displayed from the dyadic interviews impermanent creative reuse prompts. These repurposed materials included leather scraps from a local family owned business, metal recycling from a local university bike shop, and a wide variety of small "beautiful stuff" inspired found objects collected from the laboratory CDC families. The provocation was intentionally located along a spectrum of im/permanence; therefore, scissors and hot glue 
guns were made available to fix materials together. The participants were invited to collect loose parts (Daly \& Beloglovsky, 2014) from the creative reuse cultural centerfound objects that they could move, manipulate, control, and change while they worked. The participants could, combine redesign, take apart and put loose parts together in almost endless ways (Daly \& Beloglovsky, 2014).

The focus groups also had access to the multiple ateliers in the center, all filled with reuse materials that lend themselves to identity studies. There were organic snacks and drinking water available at a nearby table. Each small focus group were provided with a page listing a series of questions for the session. The participants agreed to be audio recorded throughout their conversations. During the interviews, I came and went from the rooms the groups were meeting. I recorded each pairs' conversation and was also listening in on the conversations from another room.

During this third narrative session, the two small groups sat at different tables to collaborate on the creative reuse prompt, and then joined back up in the conference room to share their storied dolls with each other in one focus group. Both small focus groups were given an identity planning chart to help make decisions and reach agreements about giving their persona doll a name, gender, age, race and ethnicity, favorite foods and activities, economic class, home, cultural background, spoken languages, religion or spirituality, and family structure. The two groups were prompted to develop stories about the doll based on a list of physical traits dyadic pairs used to describe themselves and each other during the first two narrative sessions. 
Additionally, metaphors that individuals had used during the dyadic interviews to describe their experiences of reuse identity studies were provided for the small groups to draw upon to construct their doll's identities. Throughout the process, participants selected reuse materials from the IRPP Center to make clothes, hair and accessories for the dolls. By following this process, I began to make sense of my data by deconstructing and then reconstructing narrative data. These patterns were given new life in the shape of a re-story told by participants three-dimensionally with Remida-inspired persona dolls. In other words, the participants personified two Remida-inspired educators in doll creations (see Figure 14).

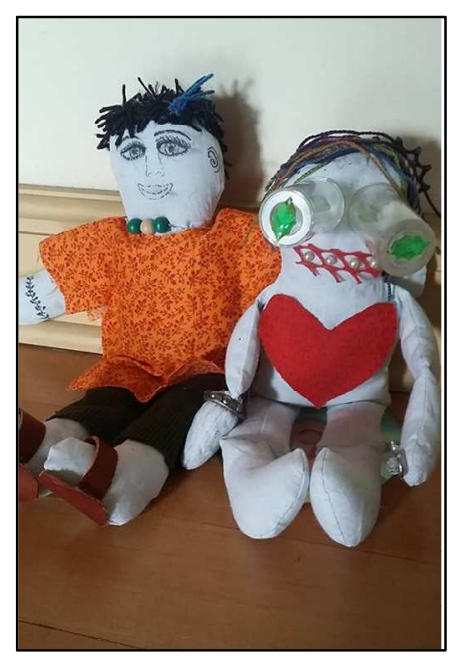

Figure 14. Remida inspired persona dolls Logan (left) and Harmony in Dissarray (right).

\section{Focus group.}

Director Joni volunteered to go first and share the cultural identities, socioeconomic status and family make-up she created with studio teacher Ann and classroom teacher Clara. Joni's laboratory CDC group chose to start off with a 'deeper story' that 
introduces an anti-bias issue of gender fluidity, "Logan does not identify on a binary of male/female and is non-binary gender. Logan uses the pronoun they rather than he or she.” Joni then shared the bi-ethnic and multi-cultural family make up her group created for the reuse persona doll, "Logan is thirty-one years old, bicultural and bilingual, and was raised by a Euro-American dad, and Chinese mom. They love biking, gardening, yoga hiking, the arts and pizza, beer, and ice-cream.” And then Joni shared their social construction of the doll's socio-economic status, shared housing and religious orientation, “Logan's parents were middle class and Logan is currently a financially struggling, early childhood teacher living in a shared house off Hawthorne. Logan loves to travel, is single, has housemates and a dog and a cat. Logan identifies as non-religious but spiritual."

Joni noted that her focus group did not use metaphors quite as much as the corporate CDC group. She shared how the experience was a great opportunity to talk about their collective experience of being in the laboratory $\mathrm{CDC}$ and what it means to come from a place of social constructivism and Remida-inspired work. Joni described her group's persona doll in concrete physical terms, "Logan is tall, slim, short haired, and has tattoos."

Both groups were asked the same prompt—How did the doll come to identify as a social constructivist and Reggio inspired educator? Joni announced her group's creation of a new teacher, another person to join their learning community and placed an immediate emphasis on the persona doll's non-binary gender pronouns. Joni shared her group's social construction of Logan, 
This is Logan, intrigued by the possibility of working in a social constructivist university-based school. Logan is a funny storyteller and an extrovert working on master's degree in early childhood development. This first-year teacher is excited about combining an advanced degree coursework alongside working directly with children. They discover many complexities in this work —an explosion of thoughts, ideas and exchanges among teachers, children and families. It is exciting, overwhelming and invigorating as well as exhausting.

When asked, how did the doll come to identify as Remida-inspired? Joni shared Logan's philosophical approach to early childhood education, "Logan sees the value in reuse materials. Having Remida as a resource, inspiration, backdrop, partner and inhouse events to go to inspires Logan to incorporate the reuse and Remida materials in the classroom."

Joni shared Logan's experience of socially constructing identity studies with children as a Remida inspired educator, "Logan feels a sense of professional satisfaction, and of the past identity of the school as an inspiration. Logan loves the idea of "not knowing', and the openness of knowing we can negotiate with children."

Maggie introduced everyone to "Harmony in Disarray" who was propped up in a sitting position on the table with the help of a used compact disc (CD) that was glued to her backside. Maggie told the group how she and classroom teacher Miranda chose her name, what gender they ascribed her, and her age, "Her name came to us from a CD that we found for her to sit on called Harmony in Disarray. She identifies as a female and is 36-years-old." The corporate CDC group did not give the doll a race/ethnicity, ethnicity, 
spirituality, economic class, or spoken languages, but they were detailed in describing her favorite foods and activities, home and family structure. Next, Joni shared a little bit more about the who the doll is though adding that Harmony lives in a non-traditional diverse family structure, "Her family lives in two different houses and includes a mom, a dad, kids, a sister and dogs." And this was followed by basic likes and dislikes and cultural identities her group created for the reuse persona doll, "Her favorite thing is to be outdoors, she doesn't eat red meat, but likes sweets and tea. She enjoys playing music and loves her work, children, tacos, chips and salsa."

Maggie began to share how the metaphorical significance of the doll's eyes, "Harmony has two layers of eyes; she was born with green eyes and she also has new eyes, for seeing different ideas and seeing things differently over time." And the doll's hair, "Her hair is a colorful rainbow of changing colors; her rainbow hair represents that a material can be one thing, and then be something else and become again and again." Then she added that the Harmony, "has a big heart and is very natural and does not dress very fancy. And she has teeth. She likes to be in control."

Maggie's description of the reuse persona doll became increasingly metaphorical and storied as Maggie described the symbolism of her watch, and magnifying lens, “Harmony's watch goes forwards and backwards in time. The watch represents how she changes over time, going back and learning from the past, and then going forward in her learning. She has a magnifying glass, to see things from a new perspective."

Trails and traces of Miranda's identity were visible on the reuse persona doll including the gears that represent what she referred to as her "busy brain" during a 
previous dyadic interview. Maggie shared, "Harmony has a busy brain — that is what there are gears in the back, she is always thinking. She is a thinker; a reflector and she is inspired by reuse and recycling centers."

Additionally, I extracted metaphors that participants had used during the dyadic interviews to describe their experiences of socially constructing identity studies with young children as a Remida-inspired educator and provided these metaphors for them to draw upon as optional prompts-What "next steps" does the persona doll want to take? What "spark" does the doll bring to her school? What is the doll's "hidden treasure?" Maggie shared, “Harmony's superpower is that she never gives up, and she is full of hope. Her hidden treasure is her hope. The spark that she brings to the school is her new eyes that see a different perspective." As a final note on the topic, Maggie told the group that the persona doll is a blend of her and Miranda and Julienne's thinking from this process and some new ideas that came up.

Thus, using reuse materials, I asked participants to consider making persona dolls that would personify their ideal of sustainability and reuse educational practices as an embodiment in the doll. One doll named Logan, chose to use gender neutral pronouns for themselves and has a persona of gender fluidity. Another doll named Harmony in Disarray, lives in a non-traditional family system where one household recycles, and reuses and the other doesn't. Since the focus group, these dolls have traveled to several schools introducing themselves to classrooms of children and opening the community to their ways of living and experiencing the world around many topics, reuse materials education, gender identity, and many more. 
Again, I use a re-storying method to summarize key points made by the Laboratory CDC focus sub-group participants and to express multiple perspectives through a collective voice and as embodied in this storied identity doll named "Harmony in Disarray."

\section{Harmony's re-story.}

I would like to introduce you to Harmony. Her name came from a CD that we found for her to sit on that says, "Harmony in disarray" so the teachers who made her decided to name her Harmony. She is a she and that is how she identified-as a female. She really likes to be in the outdoors. She doesn't eat red meat. She is inspired by reuse and recycling centers like Remida. Her family lives in two different houses. At her dad's house they recycle and reuse a lot of materials. At her mom's house they don't recycle or reuse materials and usually they throw everything in the garbage. And that is okay.

Her superpower is that she never gives up, she is full of hope the spark she brings to the school and her home is her new eyes that see the world in new and different ways.

Look closely, the eyes she was born with are green and she has the ability to see things with new eyes so that is why she has two layers of eyes. She has Rainbow hair to represents that a material can be something and then be something else and then be something else and become again and again and again, so her hair is a colorful rainbow of changing colors. Her watch goes both ways - the time goes forward and backward - sometimes she goes back and thinks about what she has learned, and other times she goes forward learning new things. She has a magnifying glass, to see things close up and far away. Harmony has a busy brain - that is what there are gears in the back, she is always thinking. She has a big heart and she does not dress very fancy.

In using a re-storying method, the corporate CDC sub-group participants express multiple perspectives through a collective voice and as embodied in this storied identity doll.

\section{Logan's re-story.}

I want to introduce a new student teacher who volunteers in the Remida center. This is Logan, who has a tattoo on their wrist. Logan loves biking and ice-cream. 
Logan is a funny storyteller and loves to talk a lot. Logan has housemates and a dog and a cat.

Logan has brought your class materials from the Remida center, but first Logan would like to ask you some questions today. When Logan asks you a question you can shake your head yes or no. If you have an answer to Logan's question you can quietly raise your hand and I will call on you to answer. Logan likes [the laboratory $\mathrm{CDC}]$ very much. Can you guess why?

There are some things about Logan's identity that Logan would like you to know. Logan feels like both a boy and a girl, so instead of calling Logan "he" or "she" we call Logan "they." Logan's mom is from China and so they speak Chinese and English.

Logan gets very excited about learning with young children and their favorite experience is sharing big ideas with teachers, children and families. Sometimes the ideas are so big they feel like explosions of thoughts in Logan's head! Logan feels lots of different feelings at school: excited, energized, and sometimes overwhelmed and super tired. Do you ever feel these feelings at school?

Logan doesn't know the answer to everything and likes to find the answers with children in the classroom. Logan likes not knowing everything because then Logan can learn new things every day. For example, Logan is learning how to reuse, recycle and compost and would like to share this with you. Logan thinks it is fun to reuse materials, and good for the earth, the water and the air to reuse and recycle materials. Do you and your family recycle materials at your house and at school?

Logan likes working with reuse materials in the Remida center and exploring ideas for how to rescue materials from the garbage and give them a new life and a new identity in the classroom.

The corporate CDC persona doll took on a very metaphorical and symbolic

identity and the Laboratory CDC persona doll was given more concrete and pragmatic characteristics. After giving the Laboratory CDC focus sub-group a chart of qualities to fill out for the persona doll who I assumed would take on a female gender, Joni commented to her group, "So we have been referring to her as female." Ann suggested that is because that their staff and the field of ECE is mostly female. Clara offered hesitantly, "Maybe she identifies as a female?" The group paused and then Joni said, 
"Did you hear that legally, non-binary is now categorized as a gender? I think we should make her-their identity." This suggestion inspired the group to give the persona doll a non-gender specific name like the ones they observed that student teachers at the CDC often select for themselves. They began borrowing names from student teachers and Joni shared how she liked a gender non-conformist name,

Because it was their chosen name. This is kind of an interesting story, we have a lot of non-binary student employees who have come in and applied for a job with one name and then once they have been here, they have asked us to call them by another great chosen name.

The group agreed that the increasing plurality of identities among the student staff was important to imbed in the reuse persona doll whom they named Logan.

\section{Reuse persona doll summary.}

In Chapter 2, I looked at Whitney's (1999) work on the power of persona dolls as one of the most amazing anti-bias tools in the early childhood classroom. Persona dolls serve a different purpose from dramatic play dolls in a classroom. When children encounter a classroom persona doll they are interacting with another member in the classroom. To achieve this, a persona doll is created whose identities remain as constant as those characteristics do for real children in the classroom and whose life experiences unfold just as they do for children in the classroom. These individual and social identities help the children connect with the dolls and make the dolls stories more powerful. This is unlike a dramatic play doll in the classroom whose age, name, family, identity and gender can change any time a child in the classroom desires to do so. 
Storytelling with persona dolls (dolls that are given names, family histories, and other traits by educators) "is a powerful tool for teaching classroom and social skills, giving children words for and tools to manage their feelings, developing problem-solving and conflict resolution skills, expanding children's comfort with difference, undoing stereotypes and biased information, and helping children to stand up against bias" (Whitney, 1999, p. 233). Inviting educators to share their own stories of working with self-portraits constructed with reuse materials provide insights to better understand how they simultaneously tell the life stories of others.

The participants' experiences of becoming Remida-inspired was captured in reuse collages, Remida-inspired documentation, and reuse persona doll creations, followed by semi-structured interview narratives. By asking Maggie, Ann, Julienne, Joni, Clara, and Miranda about their experiences with working with reuse materials around identity studies, the essence of their experience as early childhood educators could be situated in the context of their metaphors. Maple and Edwards (2010) recommended using metaphors to explore the plot of a participant's story and to unlock data. They suggested that, "Metaphors provide a mechanism to relate, compare, and make meaning of new knowledge with lived experiences" (p. 40). Engaging participant's metaphors proved to be an effective interpretive methodology.

Each participant's narrative and college are followed by a re-storying method to summarize key points made by all the participants and to express multiple perspectives through a collective voice. Lichtman's ideas inspired me to consider how re-storying is useful to interpreting narrative data based from a social constructivist perspective that 
employs successive writing and rewriting and discussing stories to promote conceptual application and critical thinking with the power of creative reuse activities in the context of identity studies.

I began to realize that the research subjects in this study included the multiple identities of the materials, the participants, the children and Remida itself as concepts together - all having the affordance of being fluid and in a constant state of metamorphosis. I began to conceptualize this study as a three-dimensional narrative that took on the form of a shapeshifting myth in which the protagonists, the materials and the theories themselves intersect within a three-dimensional narrative inquiry space in a constant state of being transformed, transformational and interdependent. In this study, I found that inviting educators to share their own stories of socially constructing persona dolls constructed with reuse materials provides insights to better understand how they simultaneously tell the life stories of others.

\section{And the Stories Continue...}

Three metaphorical archetypes emerged from the data interpretation and discussion, namely, (a) Identifying with Reggio: The Forager, (b) Identifying with Remida: The Global Homesteader, and (c) Socially Constructing Identity Studies: The Navigator. Although each participant's experiences were unique, patterns came into view which are interpreted through Katz (1972) framework for growth stages of preschool teachers: survival, consolidation, renewal, and maturity. These four growth patterns are further aligned with the forager, homesteader and navigator archetypes of becoming Remida-inspired that emerged from the creative reuse and interview process. 
The literature review in Chapter 2 specifically examined close ties between culture and issues of learning to better understand the intersections of individual and social identity factors in the context of Remida-inspired identity studies. These included (a) theories that influence praxis found within Remida Centers, (b) concepts of early childhood education for sustainability, and (c) socially constructing identities in early childhood education. Beyond the professional growth stage of becoming a Reggio-inspired educator, my research question looks at the adoption and growth cycle of learning a Remida pedagogical approach.

The four professional growth stages are devices I used to organize the data in a way that shows growth stages relevant to this adoption cycle. I have selected foraging, homesteading and navigational strategies the participants spoke about relative to the Remida adoption cycle that involve a social constructivist approach within situated learning experiences as narrative data. The Hidden Treasures, to be fully revealed in chapter 5, highlight teacher's experiences of adopting Remida materials and philosophy to foster children's social identity as global ecological citizens who are capable of understanding and expressing more complex features of social identities.

The participants mentioned ideas that alluded to straddling two cultures and moments of disequilibrium and opportunities for improvement they see during their growth cycles. They spoke of a process of deepening their level of sophistication for how they can systemically weave ideas about social identity throughout their identity studies by using the reuse materials as a vehical for this iterative process. Literature having to do 
with pedagogical adoption cycles, professional growth, reuse epistemological display in the classroom, social constructivism, and creativity as a vehical for social-identity formation, helped me to interpret how these educators think about developing socialidentity in a manner that situates the concept of self within the context of global ecological citizenship. 


\section{Chapter 5: Hidden Treasures and Implications}

Chapter 5 begins with reflections regarding how architecture as a metaphor for social constructivism is a useful tool for interpreting the narratives of educators who work with identity studies through creative reuse. Architecture as metaphor illuminates the significance of designing space and making a new culture. In this chapter, I also re-introduce the purpose of the study, including my research problem and question and briefly revisit metaphor as a methodology. As well, I reflect on the Hidden Treasures that emerged from the participants identity work and professional formation. Then, the final two sections present my study's conclusions, its implications, and final thoughts.

The women and children of Villa Cella built their first preprimary school to foster democracy out of the material rubble of post-fascist war (Barazzoni, 2005). Since then, Remida-inspired educators are redesigning spaces for ecological awareness out of the material rubble of hyper-consumerism. Exchanges between Remida inspired educators during this narrative inquiry remind me of how the first municipal preprimary school of Reggio Emilia was hand built by partisan women directly from the debris of World War II materials, which produced a cultural value of building in a sustainable way. The value all six participants placed on hands-on learning is grounded in a Deweyan theory of experience, as embodied, always in motion, and shaped and reshaped by continuous interaction among the human ecology of personal, social, institutional and cultural environments (Clandinin, 2011). 
I began to see the participants as architects and builders of early childhood education for sustainability (ECEFS) who bridge culture and ecology to continually shape how they craft Remida inspired pedagogy. Whereas the co-founders of the educational experiences of Reggio Emilia were building physical space to transmit democratic principles during the decline of fascism of Italy, contemporary Remidainspired educators are designing physical and conceptual spaces out of reuse materials to foster border crossings, intercultural experiences, and global ecological citizenship through "fostering intelligent moderation in the next generation" (Parnell, et al., 2017). Remembering back to Julienne's words, "Reuse materials are collected from homes and circles of relationships" and Miranda's thoughts "I have a dream that this place (the IRPP center), and this vision could blend with our school in a more fluid way," the global homesteader as an archetype gives rise to social identities and rootedness by rebuilding habits of place-making. This archetype also reimagines communities grounded in reusing materials which ironically, are displaced and in transit—and appreciated as objects that have the right to become something else.

Through multiple metaphors and methodologies, the participants in this study described new ways of knowing that call upon the sustainability of connection and inclusion and are profoundly creative and fluid. Rather than struggling against what they didn't want, participants turned towards what energizes and inspires them, towards what calls to them, to co-create yet another culture. These Remida inspired educators socially constructed new stories to explain the world and our participation in it, and renewed value systems with images and symbols that connect us to each other and with the global 
ecosystem. They reoriented theories about the relationship between the individual and the social by calling attention to the intercultural dynamics through which educators connect their practice to Remida and see their selves through reuse materials in a North American context. This study also serves as a portrait of two Remida inspired CDC's located in the Pacific Northwest and how they shape and are shaped by their ecological contexts.

Through narrative inquiry, I re-conceptualized identities as a source of data grounded in narrative interpretations of stories. In this study, six participants narrated their experiences of how they became Remida inspired, from their unique perspectives as directors, studio and classroom educators. The participants share stories of socially constructing sustainable spaces within their schools using alternative and reclaimed materials that they describe as impermanent, fluid, playful, hyper-open ended, transformational and healing. These are stories about renewable architecture in a perpetual process of social re-construction by educators outside of the context of Reggio Emilia, Italy who - 23 years after the construction of Remida — declare and maintain a temporal, social and spatial interest in the approach.

The purpose of this research study was to conduct a narrative inquiry into Reggioinspired early childhood educator's experiences with reuse materials in Reggio Emilia inspired identity studies. In this study, I explored the new and evolving epistemological spaces created by Remida-inspired educators, outside of the context of Reggio Emilia, Italy. Interpretive methodologies of re-storying (Lichtman, 2013) and of using metaphors to unlock data (Maple \& Edwards, 2010) proved to be effective in this narrative inquiry. 
Multiple metaphors illuminated the narrative data I collected to pursue my main research question, "What are early childhood educators' experiences with reuse materials in Reggio Emilia inspired identity studies?" An interpretive term central to this study was the metaphor of a hidden treasure. Over time, as I reflected on the dyadic interview data, I began to realize that each participant brought a unique perspective to the project and I began to call these unique points of view, Hidden Treasures. This term integrates classroom teacher Miranda's documentation inspired by Elizabeth Gilbert (2015) and the idea that we all have Hidden Treasures inside of us that we can reveal through identity work. Gilbert's (2015) idea revolves around a central question upon which she believes all creative living hinges: "Do you have the courage to bring forth the treasures that are hidden within you?"

Surely something wonderful is sheltered inside you. I say this with all confidence, because I happen to believe we are all walking repositories of buried treasure. I believe this is one of the oldest and most generous tricks the universe plays on us human beings, both for its own amusement and for ours: The universe buries strange jewels deep within us all, and then stands back to see if we can find them. (p. 8)

Narrative data revealed an image of the child as a Border crosser, capable of understanding and expressing more complex features of social identities. This was brought forth in the participants' Hidden Treasure: Children's Identities are More Fluid. Likewise, an image of the child as a global ecological citizen was brought forth in the Hidden Treasure, Remida Materials Support Constructivist Identity Studies. These 
images are supported by findings in the literature review that pre-primary age children are competent at representing their theories on identity and ecology through reuse materials (Reggio Children, 2005).

Situating the Hidden Treasures within the participants' identity studies highlighted the places where multiple experiences contributed to a Remida adoption cycle. Throughout the documentation process, I reflected more deeply on the "capacities, aspirations, longings and secret talents buried inside" (Gilbert, 2015, p. 8) of each participant's stories. As curator, I browsed through all the dyadic and focus group transcripts and made analytic memos about my first impressions. Then I re-read the transcripts again and again, one by one and line by line. Next, I labeled relevant words, phrases, sentences and sections.

Data interpretation was a process of sorting and sifting like the processes with materials in the Laboratory creative reuse cultural center (CRCC). To epistemologically display these materials in a way that is thought provoking and inherently beautiful, my original intention was to organize the narratives into neat thematic piles. I could then tuck metaphors into a basket, hang stories from a rope, and weave conceptual knots threedimensionally. Shifting my thinking entirely over time, I decided to illuminate metaphors onto a light surface and project stacks of narrative patterns onto a wall of experiences to create shadows and blur borders. Throughout the documentation process, I sorted through participant's metaphors, concepts, theories, values, and common threads. I found a way to transmit themes and key concepts through re-telling stories as a means 
of learning to think with stories (Clandinin, Caine, Estefan, Huber, Murphy \& Steeves 2015).

In section one of Chapter 5, the hidden treasures revealed through participant's identity studies are brought forth as the main resultants of the findings. In this section, the directors, studio and classroom teachers who participated in this study are reintroduced and I reflect on how their individual theories or hidden treasures unearthed multiple subjectivities. In the second half of Chapter 5, I present my study's conclusions, its implications, and my final thoughts.

Further, I found that the educators in this study experienced Remida materials as supportive of social constructivist identity studies. In re-storying of narrative data through a single voice and perspective, three metaphorical archetypes emerged, (a) identifying with Reggio: the forager, (b) identifying with Remida: the global homesteader, and (c) socially constructing identity studies: the navigator. Although each participant's experiences were unique, patterns came into view which I simultaneously interpreted through Katz (1972) framework for early childhood educator's professional growth: survival, consolidation, renewal, and maturity.

To address my main research question, "What are early childhood educators' experiences with reuse materials in Reggio Emilia inspired identity studies?" I found the following treasures — or unique points of view — to be valuable in uncovering the narratives of educators who work with reuse around identity studies. 


\section{Hidden Treasures Revealed through Identity Work}

Stories generated by the first narrative inquiry prompt: "Please share with one another the story of how you came to identify as a social constructivist, and Reggioinspired early childhood educator" elucidated participant's pedagogical roots in the Reggio Emilia approach. Participants used the metaphors strongest journey, dig into, blossoms, map all laid out, and feeding off to describe their experiences of identifying with the democratic and equity-based approach. These metaphors unlocked a forager archetype which corresponded with Katz "survival stage" where the overriding concerns are daily challenges and the primary resources are local pedagogical support. During this cycle of growth, participants gathered pedagogies, identified relationality as an adaptive strategy, searched for theoretical frameworks, and sustained situated learning. In this data, I focused on the protagonist as a forager in search of theoretical frameworks to survive their first year of teaching.

Early on in her career, director Joni's optimal foraging strategy (Roughgarden,1995) became the ability to select and combine anti-bias and Reggio inspired pedagogies that supported her growth. The Hidden Treasure, the Reggio Emilia Approach is equity-based pedagogy was sheltered inside of director Joni.

Joni shared that for her to digest a new pedagogy, it was essential that the Reggio Emilia approach integrate rather than conflict with the anti-bias values that had transformed her consciousness,

Conversely, when we actively started exploring the anti-bias work there was a lot that I had to let go of from my teaching past. It was incredibly exciting, a whole 
revolution that I was happy to be a part of, but it felt like I was going one way and now I've got to go another way. When I learned about Reggio Emilia philosophy I thought, wow! That is the way you articulate what we are trying to do, those are new ways that we can do this better, do this work powerfully, but it didn't feel like I must stop something that I am doing; change what my other work is and shift it into something else.

She described her turning point as, "When I went to the first conference workshop about Reggio Emilia schools and Reggio-inspired work, it clarified for me that Reggio inspired values were aligned with anti-bias work." Joni's paradigm shift necessitated the creation of new and evolving epistemological spaces and by reclaiming all parts of her identity, even those that clash, she escaped essentialist categories and envisioned an impermanent home where she could stand and claim her own space making a new culture-with her own lumber, her own bricks and mortar and her own feminist architecture through her story, Joni demonstrated the ability as a critical educator, to sustain contradictions and to transform ambivalence.

Joni survival as a forager resulted from gathering pedagogies and searching for theoretical frameworks that sustained her multiple professional identities. Anzaldua (1987) conceptualized critical consciousness as neplanta: An Uto-Aztecan term for the "transitional space a person occupies when experiencing transitions or the personal dichotomy that occurs between shifts of awareness" (p. 106). The process by which someone like Joni becomes awakened to a new consciousness is not easy or comfortable and though it may be a source of intense pain, its energy comes from continual creative 
motion that keeps breaking down the unitary aspect of each new paradigm.

Equity-based curriculum is an approach to ECE that sets forth values-based principles and methodology in support of respecting and embracing differences and acting against bias and unfairness. Hooks (2010) wrote, "Children are organically predisposed to be critical thinkers" (p.7). An educational element involves supporting children to socially construct their individual and social identity, find their voice, and be proud of who they are. If children feel strongly about themselves, they can then support others to develop their identity, voice, and be comfortable with diversity (DermanSparks, LeKeenan, \& Nimmo, 2015). Equity based pedagogy requires critical thinking and problem solving by both children and adults.

Early on in her career, Laboratory CDC classroom teacher Clara's optimal foraging strategy also became the ability to select pedagogies that supported her background in psychology. Clara's career began ten years ago at a Reggio inspired school in California. She used the metaphor democratic education as healing, to describe her experience of Reggio inspired philosophy as sustenance for her practice,

That is the beauty of the whole philosophy, it heals and changes people's lives. If you grow up in an environment like that, you are becoming self-confident; trusting yourself and exploring your ideas, speaking your truth because you know you have the freedom and the right to speak your mind.

The Hidden Treasure, The Reggio Emilia Approach heals through democracy, was sheltered inside of classroom teacher Clara. As mentioned at the beginning of this chapter, increasingly, ECESF is looking to global citizenship as a democratic framework. 
Rinaldi (1998) wrote, "To form a sense of self, a sense of belonging that is both local and global, to be clearly bound to the place where we live but, at the same time, to dialogue with the world is an essential value for the future" (p.79) In Australia, the national professional association for ECE has recently updated its Code of Ethics to include "the obligation for early childhood educators to "work with children to help them understand that they are global citizens with shared responsibilities to the environment and humanity' (Code 1.4)" (Davis, 2009, p. 230). This approach to fostering children's awareness of social and ecological interconnectedness is congruent with recent movements focused on the intercultural politics and pedagogies that underpin global early childhood education for sustainability (ECEFS).

The Chapter 2 literature review on early childhood education for sustainability (ECEFS) showed that researchers have written about the significance of recognizing the rights of young children (Johansson 2009; Pramling, Samuelsson, \& Kaga 2008) and the right for children to be involved in research as "competent informants of their own experience" (Danby \& Farrell, 2004, p. 43). Even more researchers have written about children as agents in their own lives or as competent citizens who contribute confidently in issues that impact on them (Clark and Moss 2011; Davis 2009). Therefore, there is a need for further discussion in ECE literature that brings together the notion suggested by Kjørholt (2005) that respecting children's rights links to competence within an early childhood context. When young children are included in making decisions that impact their lives, including decisions regarding ecological sustainability, they are capable in contributing to conversations as competent citizens that lead to purposeful action in 
caring for their environment (Mackey, 2012). To further illustrate the forager analogy, garnering the democratic theory of the Reggio Emilia approach optimized Clara's resilience as a social constructivist educator.

The second narrative inquiry prompt: Please share the story of how you came to be Remida-inspired, gave insight into participants' experiences of professional formation in the Remida approach. Participants used the metaphors coming home, flows through the veins, fine tuning, dug into, harness, footprints, groundwork, beef up, captured, transformation, spark, next layer, hitting a juncture, little bump, and dream. These metaphors unlocked a global homesteader archetype which corresponds with Katz's (1972) consolidation and renewal growth stages for early childhood educators. During this stage of growth, participants as Homesteader's plowed Remida as a geographic terrain and a terrain of consciousness, sowed early childhood education for sustainability (ECEFS) and cultivated theoretical materials. In this data set, I focused on the protagonist as a global homesteader cultivating theoretical materials to consolidate and renew their professional formation.

In my understanding of the data, the protagonist as global homesteader recognizes that, in the long run, the best way to sustain Remida-inspired reuse practice is to be selfsufficient as a community. During this stage in their professional formation as individuals in a learning community, Katz suggests that educators view their identity in an expansive sense - the data demonstrating this expansiveness as participants seeking global ecological citizenship. This requires actively working to build on their knowledge, skills, and perceptions of the meaning and process of working with Remida-inspired 
materials as a local community in an increasingly globalized world, to cultivate a homestead for creative reuse cultural education.

It was the sense of ground-breaking experience in new frontiers that led to my interpretation of the participants' professional formation through the lens of a global homesteader archetype. In retrospect, it seems important to problematize the concept of space as 'frontiers' because historically a lot of colonial frontiers weren't actually frontiers and had been previously inhabited by indigenous cultures. In this research, I have decidedly spatialized the global homesteader as Remida-inspired educators who are redesigning spaces for ecological awareness out of the material rubble of hyperconsumerism.

I emphasize the term global homesteader because I am not defining homesteading by where someone lives, such as the city or the country, but by the lifestyle choices they make rooted in their identity as a global ecological citizen. Homesteading is a lifestyle of resourcefulness characterized by subsistence agriculture, home preservation of food, and may involve small scale production. Pursued in different ways around the world — and in different historical eras-homesteading is generally differentiated from rural village living by the isolation of the homestead. Modern homesteaders often use renewable energy including solar electricity and wind power.

The Remida-inspired global homesteader is epitomized in Maggie's use of the metaphor "flows through the veins of our school" to describe the space/place of Remida and organization as a value, 
We ended up working with SCRAP in [a]... capstone class one year, who helped us put a reuse center in our school, and then it grew and grew for me. At first it was awkward because Remida-inspired education is hard to understand and to put into daily practice. People try to make creative reuse this big separate project compared to now years later, it's in the veins of our school - it flows through the veins.

Maggie's organismic metaphor supports scholarship that states the Remida creative recycling center holds a vital place in the "living system" (Cadwell et al., 2005, p. 115) of Reggio Emilia, Italy and increasingly so in global Reggio-inspired educational systems. Maggie, like Tarr (2003), described the many natural and recycled manufactured Remida materials in Reggio Emilia classrooms and how the use of materials is deeply imbedded in and integral to the life and culture of the classrooms.

The next Hidden Treasure, Epistemology of Display, was sheltered inside of director Julienne. Both Joni and studio teacher Maggie were members of the IRPP advisory committee who helped to recruit Julienne, the director of the corporate CDC, to form a second dyadic pair for the study. Joni and Julienne used to both work at the laboratory $\mathrm{CDC}$ and have enjoyed a thirty-year professional relationship. Julienne emphasized the importance of community in resilience as a social constructivist educator and used the metaphor "feeding off" of other Reggio inspired colleagues as a way to stay professionally nourished. Julienne's experience with reuse materials in identity studies highlighted what she considered an epistemology of display. 
The display of materials (organization as a value) helped participants become homesteaders by visiting Remida. Julienne shared a co-narrative with Joni of their first study tour of Remida in Italy, where she was particularly impressed by the display of materials, "Looking at the aesthetics of how they were putting things together and how they arranged them for the educators to see was amazing." She noted, "There was a lot of surplus, so many different colors and textures. I thought, Of course children and people would delight in this!" Joni added, "The value of organization and aesthetics come more with the Reggio approach, then what we would do with that same ecological value without the Reggio inspiration." According to Julienne, an epistemology of display included giving value to materials through aesthetic organization and display that honored their agency.

Further, it is also important to elaborate on the homestead as an analogy for participants' epistemologies of display through creation of Remida-inspired centers in their schools and experiences of Remida materials supporting constructivist identity studies. To plow a garden — or a creative reuse cultural center (CRCC) — the participants described needing a vision. All the participants except for Amanda had taken a study tour to the Remida Creative Recycling Center in Reggio Emilia, Italy. Maggie, Ann, and Joni had toured Remida twice and highlighted cleanliness, volume, aesthetics and organization as one of the strongest impressions. Maggie described how organized Remida was and how well they had divided out the materials, "The materials weren't purchases from catalogues, but were very clean, and clean in the way of organization." Ann also highlighted the epistemological display, "The first thing I remember walking 
through the doorway, was how organized and welcoming it was. I liked the cylinders with materials in them; rather than having big signs saying "plastic" and "metal" you could tell what materials were based on how they were organized." She added, "I was impressed by how clean it was and user friendly. I don't speak Italian, but I could go in there as a teacher and know what I can take or do by the way they had it laid out, without instructions." In this data, we see how discarded materials are valued through organization and display.

When participants were planning their creative reuse cultural center's (CRCC), they started by creating a community wish list of everything they wanted to cultivate there. Collaboration between studio teacher Maggie and director Julienne constituted a vital microsystem, Julienne said, "We had a teacher at [corporate CDC], Maggie, who was invested in trying to figure it out, and so that is how we started the center." Director Julienne used the metaphors of "harness" and "footprints" and "groundwork" to describe her experience of building community through local and global collaboration to plant and tend to a creative reuse cultural center ( $\mathrm{CRCC})$.

However, Julienne eventually recognized the need to trim the list down to the conceptual seeds that were the most important to sow. She used the metaphor captured, to describe her experience of being inspired by Remida to harvest a reuse materials philosophy, "We had already captured the piece about aesthetics and wonder, and what the materials might tell you. A big part of working with the materials after that was to get them out of the closets, accessible to the community and to see what this kind of commitment or value [they] would bring to our program." As homesteaders, they had a 
limited space and couldn't possibly cultivate everything at once, otherwise they would end up with a chaotic display that was unmanageable to steward.

Both the laboratory and corporate creative reuse cultural center's (CRCC) shared similar challenges in stewarding the space through collecting, organizing and displaying reclaimed materials. Julienne used the metaphor "beef up" to describe her experience of the pedagogy of display, "Remida discards support ideas or projects in the classrooms; we might beef up one collection or type of material that has attributes or affordances to support the work." The narratives that emerged from the director's dyadic interview illuminated how displaying repurposed materials bridges constructivist identity studies with reuse materials. For example, shelves holding materials might be placed vertically or at eye level rather than up against a wall, so that children and adults can encounter each other and the materials in a creative reuse space.

Julienne described sowing the seeds that would bear theoretical fruits and ecological design that her school values the most. She related, "Our reuse center is currently located in a central part of our school and has become a place of connection like the fish tank or the front office." Over the years, the corporate creative reuse cultural center (CRCC) has experimented with planting different seeds to compare how well those ideas and provocations take root and helped the community to thrive as an interconnected, interdependent whole.

As homesteaders the participants spent a lot of time, energy, and expense to improve the soil of their plot and to create a strong, rich, durable cultural production. The participants described adding the compost and nutrients of relationality, theoretical 
frameworks, situated learning, Early childhood education for sustainability (ECEFS), and reuse materials to fertilize a creative reuse cultural center (CRCC). The participants viewed this terrain as one that functioned as a third teacher that is conducive to engendering mental patterns and practices that "foster intelligent moderation in the next generation" (Parnell, Downs, and Cullen, 2017).

The next Hidden Treasure, Remida-inspired materials are extra-open ended, was sheltered inside of studio teacher Ann. Ann frequented the IRPP advisory committee meetings and seemed very interested in Maggie's Remida inspired studio work at the corporate $\mathrm{CDC}$. I began to think that as professional acquaintances they would make a good dyadic pair for my research. I invited Ann to join the study via email. Ann agreed to participate and to pair with Maggie, and they became the first dyadic pair.

Ann's studio at the laboratory CDC is a public area and has a visible emphasis on reuse, recyclable materials, loose parts (Daly \& Beloglovsky, 2014) and threedimensional work. Ann formed a unique point of view through her formation as a social constructivist working with discards, "I have come to really like some of these materials because they are so much more open ended for children to use them." During informal communications, she elaborated on reuse materials affordance of being "abstract' "hyper open-ended" and "extra open-ended" compared to other open-ended materials at school. Rinaldi (2006) coined the term intelligent materials referring to the languages of discard materials and the educational value of infusing Reggio Emilia principles into creative reuse education in early childhood. For example, the term affordance is used to describe how each medium, including complex reuse materials, provokes a special orientation to 
the problem to be solved, and thus assumes a critical role in the environment as the third teacher.

Everyone perceives creative reuse experiences differently, thus the entire community is enriched by a variety of perspectives. For example, children who had spent years at the laboratory school and were used to open-ended materials like blocks and Waldorf inspired dolls with no faces, would ask "What do I do with this?" when offered a reuse material because of its affordance of being an extra open-ended material.

The next Hidden Treasure, Remida Materials are paradoxically forgiving and demanding, was sheltered inside of studio teachers Ann and Maggie. Both educators observed the sense of joy participants experience when working with reuse materials to make creative representations. They agreed that the sense of joy and absence of pressure to perform positively influenced people to use their imaginations. Maggie said,

When I paint myself, I purposely go 'Picasso' — abstract, because it is going to be bad if I try to do it well, so I go cartoonish, the other direction, painting purple skin, because I feel the pressure. That is where the joy comes from, the pressure is gone; you are not going to do it badly with these reuse materials.

Ann and Maggie observed the self-consistent but habitually incompatible frames of reference for the reuse materials being equally forgiving and demanding, Ann observed, "There is a confidence level with how to introduce Remida materials. A lot of teachers don't know what to do with the reuse or loose parts (Daly \& Beloglovsky, 2014) material." Maggie argued, "That is not necessarily any different from any other material. I find reuse to be not a hundred percent approachable but more approachable." Maggie 
concluded that as a situated learning approach resolves the potential conflict, "You learn by doing!"

Ann's story about her experience of working with a practicum student in her creative reuse studio (see Chapter 4 Summary of the Session Two Dyadic Interviews) helps to understand what Ann meant by scaffolding extra open-ended learning experiences, "You have that proces — that sub-process — the teacher's need that level of scaffolding for themselves as well." The studio teacher's experience of Remida materials being both forgiving and demanding is characteristic of the paradoxes within complex identity studies.

The next Hidden Treasure, a spectrum of im/permanence, was sheltered inside of studio teachers' Ann and Maggie. Ann's master's thesis was entitled: To Glue or Not to Glue? The sustainability of impermanence would re-emerge throughout dyadic interviews in many forms and appeared to vary along a continuum of practice for the participants. The value of im/permanence came up immediately during the first reuse provocation and while Maggie modeled for children an avoidance of cutting, or gluing reuse materials in her studio, Ann valued using glue for project work alongside an integration of the impermanence of loose parts (Daly \& Beloglovsky, 2014).

The dyadic pair of studio teachers described participating in the same Remida Center mysteries of paper provocation in Italy, and each expressed different perceptions of the same event. Maggie experienced a transformation of consciousness from engaging in the hands-on project that shaped her "materials philosophy" and shared that for her, "the biggest take away was that none of the books were permanently affixed and a big 
part of my materials philosophy comes from that experience-that you can make things that feel so permanent, so beautiful and rich and yet [that thing] can be taken apart and become something else, and something else, and something else." This is important because children at the preschool age typically think in ways that are very focused on sole ownership of their creations.

The ah-ha moment that this teacher experienced related to the value of $\mathrm{im} /$ permanence became a classroom tool useful for helping children to understand the value of sharing, reusing, and viewing resources as community treasures. Importantly, this type of activity became a tool that she could use in her studio to help children understand ecological concepts; experiences that involved exploring the nature of $\mathrm{im} /$ permanence also became a tool useful for expressing creative reuse concepts with colleagues.

Ann, on the other hand, described feeling out of the zone of proximal development rather than inspired, “That was a little bit intimidating to me, I wasn't quite sure what they were going to expect us to create; for me to try to produce something that quickly in front of people who were obviously more comfortable with reuse." This narrative helps to gain insight into how Remida inspired educators articulate their experiences with the audience of each other, the participant researcher and the potentially larger audience of teacher researchers. The studio teachers' values and practice were situated along a spectrum of im/permanence.

The next Hidden Treasure, bridging the gap in early childhood education for sustainability (ECEFS), was sheltered inside of classroom teacher Miranda. Miranda 
joined the study when studio teacher Maggie suggested a transition age classroom educator from the corporate CDC who was interested in the study and could be paired with a laboratory CDC classroom educator as the third dyadic pair. Amanda had attended a workshop I facilitated as Inventing Remida Portland Project (IRPP) coordinator on identity and reuse a year prior. I sent Amanda an email recruitment letter and she agreed to join the study on the condition that the interviews be on the weekend.

Despite Miranda's excitement about Remida cultural education that she attributed to the "spark" that Maggie brought back from her experience visiting Remida in Italy, Amanda emphasized that there is more work to be done to help children understand the identities of the reuse materials. During her dyadic interview Amanda shared, "I don't think we are doing a good enough job of bridging the gap of "Where do these come from, what's the purpose, why do we have them?" They see them as exciting materials to use in their play, but I could do more justice to the environmental piece." Amanda's observation and concern resonates with the literature review findings for Early childhood education for sustainability (ECEFS) (i.e. Davis, 2009; Duhn, 2012; Duhn et al., 2010; Duhn \& Ritchie, 2014; Elliot \& Davis, 2009; Mackey, 2012).

When it comes to engaging young children critically with complex ecological issues, there is a paucity of research, methods, and curriculum and instruction resources to guide the socially constructivist early childhood educator. The journey from cognitive dissonance to resonance was shared among participants who re-cognized in the Remida approach elements of ecological social identity that was missing from their practice and schools. 
Equity based pedagogy in ECE is rooted in the image of the child as a global ecological citizen. In 1959, the United Nations General Assembly set forth its Declaration of the Rights of the Child, followed in 1989 by the Convention on the Rights of the Child. Many diversity and equity based ECE advocates around the world treat these documents as central to their work. Article 2 of this Convention makes clear that children's rights are universal. Especially pertinent for equity-based education, Article 29 declares that "education... should encourage children to respect others' human rights and their own and other cultures. It should also help them learn to live peacefully, protect the environment, and respect other people" (UNICEF, 2001, p.9). The rights of children in Article 29 directly link social and environmental sustainability and align with Agenda 21, a comprehensive plan of action on sustainable development for the twenty-first century that was drawn up after the United Nations Conference on Environment and Development (UNCED) and addresses children in sustainable development as a major group. The United States is the sole UN member not to ratify the Convention on the Rights of the Child passed nearly three decades ago.

Miranda used the metaphor of "penetrating a wall" to describe a lack of an Early childhood education for sustainability (ECEFS) framework in her creative reuse cultural center's (CRCC), "I think we have some big next steps that will make the connection with the material different." Miranda used the metaphor of layers, "The next layer of my experiences of reuse with children is I want to incorporate the philosophy and the value on the waste side of it." Then she added, "I love that idea around here [referring to Laboratory creative reuse cultural center $(\mathrm{CRCC})]$ that the material has a life. That would 
make so much sense to children." For example, Miranda discussed the idea of guiding students to respect the value of materials and explore creative possibilities for how resources can be conserved. She spoke about examining loose parts (Daly \& Beloglovsky, 2014) in a deep way in her classroom and school as the community of learners worked to find balance in their relationship with reuse materials. Miranda described encouraging children to think deeply about the finding balance between continuously creating and reusing materials, and the pleasure in creating something that is to be enjoyed on a longer-term basis.

Miranda provided the example of how her class discussed uses of shells to expand the children's sense of co-ownership and value of an item. She said, “...they want to use it [the shell] for block building or in the kitchen to do some [pretend] cooking, we are inviting them to bring it back after [they are done playing with the shell]. Then we created a separate space with ribbon, string, things that we felt like we could compromise with [rather than needing to keep it for themselves]. Thus, children are invited to see items as reusable and valuable to the entire community for a variety of uses beyond creating something that only one person uses once and then claims ownership over.

The participants function as homesteaders to create small scale creative reuse cultural center's (CRCC) in their respective laboratory and corporate CDCs that consolidate and renew their learning communities' social identities. The participants described working towards creating a sense of community in their creative reuse cultural center (CRCC), because it ensures their chances of pedagogical growth as Remida inspired educators. They continue to identify with Remida through specialists, colleague 
advice, and consultants as well as conferences, professional associations, journals, and study tours to Remida in Italy.

Instead of finding chickens, rabbits, hogs, a garden and/ or a green house on their homesteads, one finds the theoretical materials of unique paper, colored glass, broken tiles, textured fabric, smooth metals, various buttons, colored plastics, interesting containers, misprinted stickers, wooden pieces, jewelry, beads, scraps of leather, colored lids and caps. The homesteaders aim to cultivate as much of their own Remida inspired materials as possible, with their own hands, tools, and time. There is a degree of interconnectedness, and a desire to build a strong creative reuse culture that is intentional. Creative reuse cultural education is Reggio inspired and includes small groups, extra open-ended provocations, an epistemology of display, a spectrum of im/permanence, and plenty of time for children and adults to dialogue with the materials.

The final Hidden Treasures that I will reflect on next, children's identities are more fluid and Remida materials support constructivist identity studies, emerged from the Navigator archetypal stage of mature professional formation. The third narrative inquiry prompt: Please share what identity studies means to you, and how you have approached identity studies with young children as a social constructivist educator. In this data, the participants reflected on their experiences with constructivist identity studies and articulated their underlying belief structures about identity, cognition, and reuse. Participants used the metaphors fluid, infused, full range, flipping, anchoring, work backwards, imbedded, uncover, broadened scope and evolving to describe their experiences in the maturity stage of their professional growth. 
These metaphors unlocked a navigator archetype which corresponded with Katz (1972) "maturity stage" when the early childhood educator is likely to have come to terms with themselves as an educator and to have reached a comfortable level of confidence in their own competence. During this stage of growth, the navigator broadened the scope from physical to relational, voyaged backwards from "We" to "Me" and recovered a multiplicity of identity studies. Through this data set, the protagonist as a Navigator becomes a re-story of following paths that created new points of view and inspired others to travel along new roads and to develop new ways of seeing and new gazes alongside children and families.

The final Hidden Treasures, Children's Identities are More Fluid and Remida Materials Support Constructivist Identity Studies, was sheltered inside of studio teacher Maggie. Maggie - a studio teacher at the corporate CDC and member of the IRPP advisory committee - who was the first educator to agree to participate in this study. Maggie agreed to join the research study after resolving her concern that the study asked specifically for preschool educators. Maggie's response to my email screening fit the criteria of being a Remida inspired educator, so the age range was adjusted to include infant-toddler educators. In retrospect the study benefitted from that inclusion because that age group is often left out of early childhood discussions about creative reuse. Infant and toddler educators usually adapt professional development geared towards preschool teachers in order to meet the needs of their younger students. 
Maggie narrated her experiences of navigating infant and toddler identities through the inclusive lens of the term exceptionalities, and used the metaphor "fluid" to describe her perception of children's identities as compared to adults,

We did a lot with our infants and toddlers last year through inclusion-based attention on trying to identify children's exceptionalities and I would identify those as fluid; they might show a real strength in one moment and then a completely different one a moment later. Children's identity is more fluid than ours and is not so set at that age.

In the maturity stage of her practice Maggie proposed, "Everything a child does expresses their identity and supports them in learning about who they are in the world. Do they naturally excel at creating sounds and rhythms? Do they enjoy exploring light and shadow? Are they nervous about getting muddy? Afraid to meet new people?" These observations led to her unique point of view, "It is all an identity study" (Maggie, Personal Communication, December 2, 2015).

The observation that children's identities are more fluid than adults is supported by research on divergent thinking in children which is often associated with creativity (Jefferson, 2015). Studies suggest that children's divergence capability operates at a genius level, but that our ability to think divergently decreases dramatically as we become adults (Jefferson, 2015). Anzaldua's (1987) Borderlands cultural theory conceptualizes individual and social identities as interdependent and fluid (Gomez-Pena, 1993). A Borderlands framework is a useful lens to look through for interpreting the narratives of participant educators' who work with creative reuse and constructivist 
identity studies because it is a language that explains the social conditions of individuals with hybrid identities which transcends binary constructions.

Borderlands theorist Anzaldua (1987) observed that the Border crosser "constantly has to shift out of habitual formations; from convergent thinking, analytical reasoning that tends to use rationality to move toward a single goal (a Western mode) to divergent thinking, characterized by movement away from set patterns and goals and toward a more whole perspective, one that includes rather than excludes" (p. 101). In the process of collecting and interpreting data from six Remida-inspired participants, I recognized their identity studies as socially constructions of individual selfhood "me" within the context of social selfhood "we."

Rinaldi (2006) uses the words image and identity interchangeably and underscores how our definition of the image or identity of the child is above all a cultural, social and political convention. She stated, "What we believe about children thus becomes a determining factor in defining their social and ethical identity, their rights and the educational context offered to them" (p. 65). Through the three re-stories, the data demonstrated that social constructivist ECE is identity studies and shows that Reggio Emilia-inspired education is constructivist identity studies. Miranda described her ongoing navigation this way, “As far as identity studies in early childhood, it isn't always going to look the same. What we find out about each other each time is just going to be different and keep evolving." Strong identification with Remida led to the formation of a more sensitively attuned internal compass that steered the participants' experiences with reuse materials in Reggio Emilia inspired identity studies. 
In dyadic session two, studio teacher Maggie shared her Remida-inspired documentation entitled, Looking Through. She journaled observations of infants and toddlers looking through multiple discarded transparent bowls of different colors. In her journal, she reflected, "What that says about—entity—when you are literally changing the way that you see the world and experimenting with that." Maggie's documentation made visible how seeing with multiple perspectives signifies a democratic and equitybased identity study. Towards the end of narrative session one, Maggie shared, Open ended materials, loose parts and Remida play into constructivist conversations with children about identities. Often children's toys are so gender specific — specific colors, specific tasks that don't allow for freedom of thought or the evolution that children need to go through. Remida materials allow children flexibility of thought which supports a million different things, but it really supports identity studies.

Alongside the research participants in this study, I reached a deeper understanding of how Remida is practiced in social constructivist sites where dominant constructions of early childhood identity can be contested in creative ways that honor children's culture and learning.

In summary, the following table records the archetypes and corresponding Katz's stages that revealed the Hidden Treasures sheltered in early childhood educators' experiences with reuse materials in Reggio Emilia inspired identity studies. You will notice that column one Archeype and Katz's stages matches up with column two Hidden Treasures. 


\begin{tabular}{|c|c|}
\hline Archetype and Katz's Stages & Hidden Treasurers \\
\hline $\begin{array}{l}\text { Forager archetype which corresponded } \\
\text { with Katz (1972) survival stage where the } \\
\text { overriding concerns are daily challenges } \\
\text { and the primary resources are local } \\
\text { pedagogical support. }\end{array}$ & $\begin{array}{l}\text { - The Remida Approach is Equity } \\
\text { Based Pedagogy } \\
\text { - The Reggio Emilia Approach } \\
\text { Heals through Democracy }\end{array}$ \\
\hline $\begin{array}{l}\text { Global Homesteader archetype which } \\
\text { corresponds with Katz's (1972) } \\
\text { consolidation and renewal growth stages } \\
\text { where the educator's overriding concerns } \\
\text { are specific challenges and professional } \\
\text { renewal. }\end{array}$ & $\begin{array}{l}\text { - } \quad \text { Epistemology of Display } \\
\text { - } \quad \text { Remida-inspired materials are } \\
\text { extra-open ended } \\
\text { - } \quad \text { Remida Materials are } \\
\text { Paradoxically Forgiving and } \\
\text { Demanding } \\
\text { - } \quad \text { A Spectrum of Im/permanence } \\
\text { - } \quad \text { Bridging the Gap in Early } \\
\text { Childhood Education for } \\
\text { Sustainability (ECEFS) }\end{array}$ \\
\hline $\begin{array}{l}\text { Navigator archetype which corresponds } \\
\text { with Katz (1972) maturity stage where } \\
\text { the overriding concern is addressing the } \\
\text { larger picture and the primary resources } \\
\text { needed are seminars, institutes, degree } \\
\text { programs, books, journals and } \\
\text { conferences. }\end{array}$ & $\begin{array}{l}\text { - Children's Identities are More } \\
\text { Fluid } \\
\text { - Remida Materials Support } \\
\text { Constructivist Identity Studies }\end{array}$ \\
\hline
\end{tabular}

Table 2: Archetypes and Katz Stages and Hidden Treasures

In narrative session three, I prompted a reuse activity-oriented prompt to generate three-dimensional documentation in the form of two persona dolls to maximize the social dynamic inherent to the experience of being in a focus group. Whitney's (1999) work on the power of persona dolls as an anti-bias tool in the early childhood classroom informs this section, 
Storytelling with persona dolls (dolls that are given names, family histories, and other traits by educators) is a powerful tool for teaching classroom and social skills, giving children words for and tools to manage their feelings, developing problem-solving and conflict resolution skills, expanding children's comfort with difference, undoing stereotypes and biased information, and helping children to stand up against bias. (p. 233)

In conclusion, the unique points of view that emerged from my inquiry question ("What are early childhood educators' experiences with reuse materials in Reggio Emilia inspired identity studies?") demonstrated that inviting participants to share their life stories with reuse self-portraits provided insights to better understand how they simultaneously tell the life stories of others, which in turn influences their own life stories.

\section{Implications}

This study generates a new question: How might we work with Reggio inspired educators who are adopting a Remida lens in novel ways across Katz's stages and metaphors of professional growth? In my understanding of the data, Remida-inspired educators professionally form from identifying with Reggio Emilia philosophies as foragers. Then, they progress through identifying with Remida as global homesteaders to socially constructing identity studies with reuse materials as navigators. At a navigator stage, participants synthesized metaphors and social constructivist approaches to identity studies. Other researchers could investigate if growth patterns in the Remida adoption cycles remain consistent with both Katz's stages and the corresponding archetypal stages 
of growth identified in this study. I am inclined to believe that archetypal growth patterns don't change, but the corresponding metaphors do, thus generating new hidden treasures. Such new hidden treasurers can also be deemed unique theories and insights from Remida-inspired identity studies with diverse educator participants.

There were limitations to this study and ways for other researchers to go in new directions. Except for classroom teacher Clara, this study was undertaken with educators who predominantly identify as White, European-American, heterosexual, middle class, cis-female, monolingual, U.S citizens, and as highly educated. Clara was born and raised in Brazil, speaks English as a second language, and identifies as a woman of color.

Future researchers could recruit early childhood educators' who identify as historically marginalized by race, gender, class, nationality, and as Border crossers and utilize a narrative inquiry approach to their experiences with reuse materials in Reggio Emilia inspired identity studies. This research could engage their stories more deeply with the four tenets of post-positivist realist identity theory: 1) Identities are both constructed and real; 2) Identities are mediated through cognitive and social processes; 3 ) Knowledge garnered in the context of oppression should be afforded epistemic privilege; and 4) The power of individual and collective agency should be part of discussions of identity (Gilpin, 2006).

From a cross disciplinary perspective, this identity work affects the lives of adults who work with children in multiple ways and has implications beyond Reggio inspired early childhood education. This research supports the idea that adults and children have the right to express complex identities with extra open-ended materials that "foster 
intelligent moderation in the next generation" (Parnell et al., 2017). This liberating work moves away from dichotomies and is applicable to social workers, educators, and counselors whose lives are touched by children in systems such as child welfare, child and youth mental health, education, health care, and community-based services. Creative reuse persona dolls can be used by child and youth care practitioners who hold multiple perspectives and focus on equity, diversity and inclusion in the context of child and family counseling as well as by early childhood educators who practice inquiry and playbased approaches.

Social workers and counselors who work in multi-service model family resource programs can implement creative reuse cultural education into relational practice in child and youth care settings such as group homes, foster care, hospitals, and schools with children who express complex identities. Creative reuse identity work can benefit such programs with a strong focus on the experiences of Black youth, indigenous youth, young people identifying as 2SLGBTQ+ and young people with disabilities. Creative reuse cultural identity work has applications in undergraduate and graduate programs which focus on young children's social learning and formation from birth to eight years of age, in the context of family studies, community studies, education, research and policy. Creative reuse cultural education can support the emergence and expression of innovative and diverse perspectives in equity-based social work, early childhood curriculum and instruction, and child and family studies.

Hands-on creative reuse can foster professional identity formation in emerging child and youth care practitioners and scholars who practice indigenous methodological 
approaches to child and family studies and collaborate with indigenous communities. Educators, social workers and counselors who already employ persona dolls in their practice can integrate ecological characteristics into their persona doll work that fosters cultural values imbedded in land-based and place-based early childhood education for sustainability linked to environmental justice.

Creative reuse self-representation collage work can benefit organizational and departmental professional development and relationship building among non-tenure and tenure-stream instructors and faculty members, as well as full and part time undergraduate and graduate students. Reuse self-portrait and paired representations can serve as relational icebreakers during in-service retreats for organizations whose staff contribute to existing research and teaching by bringing innovative and diverse perspectives and experiences to the work of supporting children and family systems. This research touches on multiple, shifting and complex identity formation for all of usfrom the Forager state first year educator, social worker, counselor - to the Navigator state of professional formation and encourages professional consolidation and renewal through Global Homesteading across disciplines.

As my participants expressed, "Everything is an identity study." Novelist Chuck Palahniuk (2004) extends this idea in his observation, "Everything is a self-portrait." He continues, "A diary. The lining of your stomach is a document. The calluses on your hand tell all your secrets. Your teeth give you away. Your accent. The wrinkles around your mouth and eyes. Everything you do shows your hand.” (p. 12). I extend this idea to include our research, work, teaching, and our creativity as expressions of our complex 
identities and as opportunities to integrate creative reuse cultural education in our collective work with children, families and communities.

New metaphors and archetypal formations that emerge from an inquiry with Border crossers could generate new drafts and designs that foster new material philosophies from the debris of post hyper-consumerism. The Bordering of Remida is not a model, it is a working draft of identity contexts with reuse materials to inspire the adoption of a Remida inspired lens in professional formation in Reggio Emilia inspired education. In order to complete this research, it seems most notable to return to a re-story point of view on this "we" study. By sharing a final re-story, I believe to bring this community's voice to the fore, helping to redefine "me" through this "we."

\section{A re-story: The RE-searcher.}

Reflecting on her own Chicana Bordering with Remida, she came full circle as a co-researcher. Mindful of the brutality undocumented im/migrants experience from trespassing the social and geographical construct of "Nation States," and that by 2050 there will be 100 million climate refugees globally, she wanted to be conscientious in how she expressed her psychic experience of being an "edgewalker" - the product of genuine and thoughtful cultural crossing. As a Third Culture Kid, raised in Spain by a Mexican National and German-American, she has identified as American/ $a$, first generation Mexican-American, Chicana, Hispanic, White-Hispanic, and Gabachicana-- a hybrid identity self-constructed using the Spanish term for an Anglo-American female: Gabacha and Chicana.

She googled Anglo-Mexican and found few references to this hyphenated identity. She inhabited the space between the hyphen in Mexican-American, and between the hyphen in Anglo-Mexican. Being an Anglo-Mexican is a lonely space with few stories to sustain her; identity scholarship by Anglo-Mexican writers is scarce However, the fastest growing inter-ethnic coupling is among white European Americans and Latino/as.

She foraged for theoretical sustenance in Chicana/os studies, where there is a longer trajectory of critical race scholarship, Border Studies, and documentation and deconstruction/reconstruction of identities. She foraged for theoretical sustenance in Education for Sustainability and resolved to dig deeper and wider; 
linking the subversive kin of culture and ecology through connecting the personal to the cultural. During her dissertational research she survived her first year as a Reggio-inspired educator. During the consolidation and renewal stages of her professional growth, she collected narrative data while homesteading a creative reuse cultural education center in a Remida-inspired laboratory CDC.

As she navigated the interpretive stages of the narrative inquiry of participants' shared stories, she began to realize that the research subjectivities in the study included the multiple identities of the materials, the participants, the children and Remida itself as a holistic concept - all having the affordance of being fluid and in a constant state of metamorphosis. She began to conceptualize this study as a three-dimensional narrative that took on the form of a shapeshifting myth in which the protagonists, the materials and the theories themselves intersected within a three-dimensional narrative inquiry space in a constant state of being transformed, transformational and interdependent. In this identity study, she found that inviting educators to share their own stories of socially constructing persona dolls constructed with reuse materials, provided insights to better understand how they simultaneously tell the life stories of others. She hopes that her interpretation of the literature and data that have shaped her Remida-inspired identity studies through a Borderlands lens will act as a liberating force for others to contemplate their own stories of ecological and cultural identity formation and cognition.

The Hidden Treasure sheltered inside of her was Reggio inspired educators adopt a Remida lens in novel ways across Katz's stages and metaphors of professional growth that generate new material philosophies. Her Big Magic or creative process was re-documenting multi-metaphorical stories of global cooperation that shed light on a common desire for biocultural identities and belonging, in a world threatened by ecological, economic, and cultural degradation. Instead of chickens, rabbits, or a green house on their homesteads, participants harvested the theoretical materials of colored glass, broken tiles, discard fabric, salvage metals, repurposed plastics, misprinted stickers, found wooden pieces, jewelry, scraps of leather, colored lids and caps. The global homesteaders aimed to cultivate as much of their own Remida inspired materials as possible, with their own hands, tools, and time.

Participants' stories valued Remida places/spaces. There was a degree of interconnectedness, and a desire to build a strong creative reuse culture that was intentional. Creative reuse cultural education was Reggio inspired and included small groups, extra open-ended provocations, an epistemology of display, a spectrum of im/permanence, and plenty of time for children and adults to dialogue with the materials. Generated out of the co-construction of participant stories, her superpower is optimism that these matters of perspective will inspire early childhood education for sustainability and contribute to increasing global cooperation for a habitable planet. 


\section{References}

Abramson, S. (2004). One, two.. a hundred languages: Semiotic competence and the Reggio Emilia Approach. Innovations in Early Childhood Education: The International Reggio Emilia Exchange, 11(4), 6-16.

Aina, O \& Cameron, P. (2011). Why does gender matter? Counteracting stereotypes with young children. Dimensions of Early Childhood, 39(3), pp. 11-19.

Anzaldua, G. (1987). Borderlands, la frontera: The new Mestiza. San Francisco: Aunt Lute Books.

Anzaldua, G. (Ed.). (1990). Making soul/haciendo caras: Creative and critical perspectives by women of color. San Francisco: Aunt Lute Books.

Anzaldua, G., \& Keating, A. (2002). This bridge we call home: Radical visions for transformation. New York: Routledge.

Alarcón, N. (1996). Anzaldúa’s frontera: Inscribing gynetics. In L. Smadar \& T. Swedenburg (Eds.), Displacement, Diaspora, and Geographies of Identity. (pp.41-52). Durham: Duke UP.

Allport, G. (1937). Personality: A psychological interpretation. New York: Holt, Rinehart, \& Winston.

Ashenden, S. (2002). Policing perversion: The contemporary governance of paedophilia. Cultural Values, 6(1-2), pp. 197-222.

Barron, I. (2011) The shadows of difference: Ethnicity and young children's friendships. Race Ethnicity and Education, 14(5), 655-673.

Barazzoni, R. (2005). Brick by brick: The history of the "XXV Aprile” People's Nursery School of Villa Cella. Reggio Children, Reggio Emilia, Italy.

Barratt, R., Barratt Hacking, E., \& Pat, B. (2014). Innovative approaches to early childhood education for sustainability case studies from the field. In J. Davis \& S. Elliot (Eds.), Research in Early Childhood Education for Sustainability International Perspectives and Provocations. UK: Routledge.

Barrat Hacking, E., Barrett, R., \& Scott, W. (2007). Engaging children: Research issues around participation and environmental learning. Environmental Education Research, 13(4), 529-544. 
Beardsworth, R. (1996). Derrida and the political. New York: Routledge.

Beck, C., Freese, A. R., \& Kosnik, C. (2004). The preservice practicum: Learning through self- study in a professional setting. In J. J. Loughran, M. L. Hamilton, V. K. LaBoskey, \& T. Russell (Eds.), International Handbook of Self-Study of Teaching and Teacher Education Practices (pp. 1259-1293). The Netherlands: Kluwer Academic Publishers.

Bennet, M. (2011). Children's social identities, Infant and Child Development 20(4), pp. 353-363.

Bennet, M. (2004). Children and social identity. The British Psychological Society, 17, 512-514.

Bennet, M., \& Sani, F. (2004). The development of the social self. New York: Psychology Press.

Berger, K.S. (2008). The developing person through childhood and adolescence. New York: Worth.

Bowen, M. (1978). Family therapy in clinical practice. New York: Aronson.

Brewer, M.B., \& Gardner, W. (1996). Who is this 'we'? Levels of collective identity and self- representations. Journal of Personality and Social Psychology, 71(1), 83-93.

Bronfenbrenner, U. (1979). The ecology of human development: Experiments by nature and design. Cambridge: Harvard University Press.

Brooker, L., \& Woodhead, M. (Eds.). (2008). Developing positive identities: Diversity and young children. Early Childhood in Focus (3). Milton Keyes: Open University.

Brooks-Gunn, J., \& Lewis, M. (1979). Social cognition and the acquisition of self. New York: Plenum Press.

Brown, J.S., Collins, A., \& Duguid, P. (1989). Situated Cognition and the culture of learning, Educational Researcher, 18, 32-42.

Bruner, J. (1986). The culture of education. Massachusetts: Harvard University Press.

Burman, E. (1994). Deconstructing developmental psychology. New York: Routledge. 
Cadwell, L., Ryan, G., \& Schwall, C. (2005). The atelier: a system of physical and conceptual spaces. In L. Gandini. L. Hill., L. Cadwell \& C. Schwall (Eds.), In the Spirit of the Studio: Learning from the Atelier of Reggio Emilia (pp. 144-168). New York: Teachers College Press.

Caldwell, L. (2003). Bringing learning to life: A Reggio approach to early childhood education. New York: Teachers Press.

Cajete, G. (1994). Look to the mountain: An ecology of indigenous education. Kivaki Press.

Carlo, G., Crockett, L., \& Carranza, M.A.(Eds.). Health disparities in youth and families: Research and applications. New York: Springer.

Carlson, N. (1990). I like me! Puffin Books.

Castillo, A. (1994). Massacre of the dreamers: Essays on Xicanisma. Penguin Group.

Cavendish, LM. (2011). Stories of international teachers: a narrative inquiry about culturally responsive teaching. PhD thesis, University of Iowa. Retrieved from https://doi.org/10.17077/etd.zzu3wf2p

Ceppi, G. and Zini, M. (1998). Children, spaces, relations: Metaproject for an environment for young children. Reggio Emilia, Italy: Reggio Children.

Cerulo, K. (1997). Identity construction: New issues, new directions. Annual Review of Sociology, 23, pp. 385-409.

Chaille, C. (2008). Constructivism across the curriculum in early childhood classrooms: Big ideas as inspiration. Boston: Allyn \& Bacon.

Chang, H. (2008). Autoethnography as method: Raising cultural consciousness of self and others. Left Coast Press.

Chawla, L. (2007). Childhood experiences associated with care for the natural world: A theoretical framework for empirical results. Children, Youth and Environments, 17(4), 144-170.

Chawla, L. (2009). Growing up green. Journal of Developmental Processes, 4(1), 6-23.

Chrisman, K., \& Couchenour, D. (2002). Human sexuality development: A guide for early childhood educators and families. D.C: NAEYC. 
Clandinin, J. (2007). Mapping a methodology. Canada: Sage Publication.

Clanidinin, D.J., Huber, J., \& Steeves, P. (2011). Becoming a narrative inquirer: Learning to attend within the three-dimensional narrative inquiry space. In S. Trahar (Ed.), Learning and Teaching Narrative Inquiry: Travelling in the Borderlands. John Benjamins Publishing.

Clandinin, J., Caine, V., Estefan, A., Huber, J., Murphy, M.S., Steeves, P. (2015). Places of practice: Learning to think narratively. Narrative Works, [S.I.\}, mar. 2015. ISSN 1925-0622.

Clandinin, D. J., \& Huber, J. (2016). Narrative inquiry. In B. McGaw, E. Baker, \& P. P. Peterson (Eds.), International Encyclopedia of Education. New York: Elsevier.

Clark, A. \& Moss, P. (2011). Listening to young children: The mosaic approach. JKP.

Colucci, E. (2007). "Focus groups can be fun": The use of activity-oriented questions in focus group discussions. Qualitative Health Research, journals.sagepub.com.

Connelly, F.M., \& Clandinin, D.J. (1990). Stories of experience and narrative inquiry. Educational Researcher, 19(5), pp. 2-14.

Cooley, C.H. (1902). Human nature and the social order. New York: Scribners.

Copple, C., \& Bredekamp, S. (Eds.). (2009). Developmentally appropriate practice in early childhood programs serving children from birth through age 8. Washington, DC: NAEYC.

Cresswell, J.W. (2015). Qualitative research and inquiry design: Choosing among five research designs. California: Sage.

Curl, J. S. (2006). A dictionary of architecture and landscape architecture. Oxford University Press. ISBN $\underline{0-19-860678-8}$.

Dahlberg, G., \& Moss, P. (2005). Ethics and politics in early childhood education. New York: Routledge.

Dahlberg, G., Moss, P., \& Pence, A. (1999). Beyond quality in early childhood education and care: Postmodern perspectives. London: Falmer Press.

Danesi, M. (2000). Semiotics in language education. University of Toronto Press. 
Davis, J. (2009). Revealing the research 'hole' of early childhood education for sustainability: A preliminary survey of the literature, Environmental Education Research, 15(2), 227-241.

Delgado Bernal, D. (1998). Using a Chicana feminist epistemology in educational research. Harvard Educational Review, 68, 555-582.

Delgado Bernal, D., Elenes, C.A., Godinez, F.E., Villenas, S. (Eds.). (2006). Chicana/Latina education in everyday life: Feminista perspectives on pedagogy and epistemology. State University of New York Press.

Derrida, J. (1967). Of grammatology. Trans. by G.C. Spivak. Baltimore: Johns Hopkins University Press

Devries, R, and Kohlberg, D. (1987). Constructivist early education: Overview and comparison with other programs. Washington: National Association for the Education of Young Children.

Dewey, J. (1938). Experience and education. Kappa Delta Phi.

Escobar, A. (2011). Encountering development: The making and unmaking of the Third World. Princeton University Press.

Donadey, A. (2007). Overlapping and interlocking frames for humanities literary studies: Assia

Djebar, Tsitsi Dangarembga, Gloria Anzaldúa. College Literature 34(4), 22-42.

Duhn, I. (2012). Making 'place' for ecological sustainability in early childhood. Environmental Education Research, 18 (1), pp. 19-29

Duhn, I., \& Ritchie, J. (2014). Making 'eco-waves': Early childhood care and education sustainability practices in Aoteroa New Zealand. Children, Youth and Environments, 24(2), 123-145.

Duhn, I., Bachmann, M., \& Harris, K. (2010). Becoming ecologically sustainable in early childhood education. Early Childhood Folio, 14(1), 2-6.

Dunn, L., \& Kontos, S. (1997). What have we learned about developmentally appropriate practice? Young Children, 52, 4-13.

Durkin, K. (1995). Developmental social psychology: From infancy to old age. 
Blackwell Publishing Ltd

Eckhoff, A., \& Spearman, M. (2009). Rethink, reimagine, reinvent: The Reggio Emilia approach to incorporating reclaimed materials in children's artworks. Art Education, 62(2).

Eckhoff, A., Hallenbeck, A., \& Spearman, M. (2011). A place for the arts: Lessons learned from an afterschool art experience with reclaimed materials. Afterschool Matters, 14(1), 38-48.

Eco, U. (1976). A theory of semiotics. Indiana University Press.

Edwards, C., Gandini, L., \& Foreman, G. (Eds.). (1998). The hundred languages of children: Reggio Emilia approach - advanced reflections. Greenwich, CT: Ablex Publishing Corp.

Edwards, C., Gandini, L., \& Foreman, G. (Eds.). (2012). The hundred languages of children: The Reggio Emilia Experience in transformation. Greenwich, $\mathrm{Ct}$ : Ablex Publishing Corp.

Egan, K. (1988). Primary understanding: Education in early childhood. New York: Routledge.

Elenes, A. (2011). Transforming borders: Chicana/o popular culture and pedagogy. Maryland: Lexington Books.

Elliot, S., \& Davis, J. (2009). Exploring the resistance: An Australian perspective on educating for sustainability in early childhood. International Journal of Early Childhood, 41(2), 65-77.

Erikson, E. (1950). Childhood and society. New York: W.W Norton \& Company Inc.

Erikson, E. (1968). Identity: Youth and crisis. New York: W.W Norton \& Company. Inc.

Esmeralda de los Santos, H.V. (2005). Chicana identity construction: Pushing the boundaries. Higher Education, 4(2), 102-113.

Esteva, G \& Prakash, MS. (1992). Grassroots resistance to sustainable development: Lessons from the banks of the Narmada. Ecologist, 22(2), 45-51.

Ets, M.H. (1978). Just me. Puffin Books. 
Ezban, M. (2012). The trash heap of history, Places Journal, retrieved from: https://placesjournal.org/article/the-trash-heap-of-history/

Firat, A. (2013). Consumption, consumer culture and consumer society. Journal of Community Positive Practices 13(1), 182-203.

Forman, G. (1994). Different media, different languages. In L.G Katz \& B. Cesarone (Eds.), Reflections on the Reggio Emilia Approach (pp. 41-54). Chicago: ERIC Clearing House On Early Childhood Education.

Foucalt, M. (1980). Power/knowledge. Harvester: Wheatsheath.

Fraser, S. (2000). Authentic childhood: Experiencing Reggio Emilia in the classroom. Canada: Nelson.

Freire, P. (2001). Freedom: Ethics, democracy and civic courage. Maryland: Rowman and Littlefeld.

Freire, P. (1970/2003) Pedagogy of the oppressed. New York: Continuum.

Freire, P. (1982). Education for critical consciousness. New York: The Continuum Publishing Co.

Freire, A., \& Macedo, D. (Eds.). (1998). The Paulo Freire reader. New York: Continuum.

Freud, S. (1930). Civilization and its discontents. London: Penguin.

Froebel, (1826). The education of man. New York: A. Lovell and Company.

Fu, V.R., Stremmel, A.J., \& Hill, L.T. (2002). Teaching and learning: Collaborative exploration of the Reggio Emilia approach. Ohio: Merrill, Prentice, Hall.

Fyfe, B. (2012). Ways forward towards a socially just and humane world. Sage Journal, 41(4), 585-597.

Galindo, R. (2007). Voices of identity in a Chicana teacher: Occupational narratives of the self. The Urban Review. 39(3), 251-280.

Gandini, L. (1998). Education and caring spaces. In C. Edwards, L. Gandini, \& G. Forman (Eds.), The Hundred Languages of Children. CT: Ablex.

Gandini, L., \& Edwards, C.P. (2001). Bambini: The Italian approach to infant/toddler care. New York: Teachers College Press. 
Gandini, L. \& Kaminsky, J.K. (2005). Remida, the creative recycling centre in Reggio Emilia: An interview with Elena Giacopini, Graziella Brighenti, Arturo Bertlodi and Alba Ferrari. Innovations in Early Education: The International Reggio Exchange, 12(3), 1-13.

Gallacher, L. \& Gallagher, M., (2008). Methodological immaturity in childhood research? Childhood, 15(4), 499-516.

Gardner, H. (1983). Frames of mind: The theory of multiple intelligences. Basic Books.

Gerber, M. (2008). Your self-confident baby. Turner Publishing Company.

Gilbert, E. (2015). Big magic: Creative living beyond fear. Penguin Publishing Group.

Gilpin, L.S. (2006) Postpositivist realist theory: Identity and representation revisited. Multicultural Perspectives, 8(4), 10-16.

Giraldo, E., \& Colyar, J. (2012). Dealing with gender in the classroom: A portrayed case study of four teachers. International Journal of Inclusive Education, 16(1), 25-38.

Golomb, C. (2002). Child art in context: A cultural and comparative perspective. American Psychological Association.

Golomb, C., \& Mckormick, M. (1995). Sculpture: The development of three-dimensional representation in clay. Visual Arts Research, 21(1), 35-50.

Gomez-Pena, G. (1993). Warrior for Gringostroika. Graywolf Press, ISBN 1-55597-1997.

Gonzales-Mena, J. (2008). Diversity in early care and education: Honoring differences. Boston McGraw Hill.

Grace, D.M., David, B.J., \& Ryan, M.K. (2008). Investigating preschoolers' categorical thinking about gender through imitation, attention, and the use of self-categories. Child Development, 79(6), 1928-41.doi: 10.1111/j.1467-8624.2008.01234.x.

Grieshaber, S., \& Cannella, G.S. (2001). Embracing identities in early childhood education: Diversity and possibilities. New York: Teachers College Press.

Guerra, M. (2013). Unconventional materials at school: Teaching experiences and educational potential, RELAdEI (Revista Latinoamericana de Educacion Infantil), 2(1), 105-121. 
Guerra, M., \& Zuccoli, F. (2012). Finished and unfinished objects: Supporting children's creativity through materials. Procedia-Social and Behavioral Sciences, 51,721727.

Guerra, M. \& Zuccoli, F. (2013). Unusual materials in pre and primary schools: Presence and actions. Procedia-Social and Behavioral Sciences, 116, 1988-1992.

Hames-Garcia, M. (2011). Identity complex: Making the case for multiplicity. University of Minnesota Press.

Harter, S. (1998). The development of self-representations. In W. Damon \& N. Eisenberg (Eds.), Handbook of Child Psychology: Socialization, Personality, and Social Development, 4(3), pp. 553-617. NewYork: John Wiley \& Sons.

Haslam, S. A., van Knippenberg, D., Platow, M., \& Ellemers, N. (Eds.) (2003). Social identity at work: Developing theory for organizational practice. New York and Hove: Psychology Press.

Hawkins, F.P. (1997). Journey with children: The autobiography of a teacher. University Press of Colorado.

Helm, L. \& Parnell, W. (2010) Learning in a Reggio-inspired reuse center, Networks: An online research journal for teacher research, 1(2), 3-12.

Henderson, B., Meier, D.R., Perry, G., \& A.J. Stremmel. (2012). The Nature of Teacher Research. In G. Perry, B. Henderson, \& D.R. Meier (Eds.), Our Inquiry, Our Practice: Undertaking, Supporting, and Learning from Early Childhood Teacher Research(ers). Washington, DC: NAEYC.

Heroman, K., Trister Dodge, D.T., Berke, K., Bickart, T., Clker, L., Jones, C., Copley, J \& Dighe, J. (2010). The creative curriculum for preschool. Teaching Strategies for Early Childhood.

Herrera-Sobek, M. (2006). Gloria Anzaldúa: Place, race, language, and sexuality in the magic valley. PMLA, 121(1), 266-271.

Hicks, D., \& Holden, C. (Eds.). (2007) Teaching the global dimension: Key principles \& effective practice. London: Routledge

Hobart, C., Frankel, J., \& Walker, M. (2009). A practical guide to child observation and assessment. Cheltenham: Stanley Thornes Publishers.

Hobba, M. (2006). ReMida Denmark: Thoughts from Australia. Challenge, 27-28. 
Horton, J., Hadfield-Hill, S., Christensen, P., \& Kraftl, P. (2013). Children, young people and sustainability: Introduction to special issue. Local Environment: The International Journal of Justice and Sustainability, 18(3), 249-254.

Humes, D. (2013). Garbology: Our dirty love affair with trash. U.S.A: Penguin Books.

Hunter, S.V. (2010). Analyzing and representing narrative data: The long and winding road. Current Narratives, 2, 44-54.

Hutchins, E. (1995). Cognition in the wild. MIT Press. ISBN 0-262-58146-9.

Iorio, J.M., Hamm, C., \& Parnell, W. (2017). Place, matters of concern, and pedagogy: Making impactful connections with our planet. Journal of Early Childhood Teacher Education, 38(2), 121-135.

Jans, M. (2004). Children as citizens: Toward a contemporary notion of child participation. Childhood, 11(1), 27-44.

Jefferson, A. (2014) Genius in France: An idea and its uses. Princeton University Press.

Johansson, E. (2009). The preschool child of today - the world citizen of tomorrow? International Journal of Early Childhood, 41(2), 79-95.

Joseph, J. E. (2012). Saussure. Oxford University Press.

Jung, C.G. (1969). Man and his symbols. Doubleday.

Kaminski, J.A., \& Gandini, L. (Eds.). (2002). Innovations in early education: The international Reggio Exchange. 9(1-4).

Kant, I. (1959) Critique of pure reason. London: Dent/Everyman.

Katz, L.G. (1993). Distinctions between self-esteem and narcissism: Implications for practice. Perspectives from Eric/EECE: A Monograph series, No. 5.ERIC Clearinghouse on Elementary and Early Childhood Education.

Katz, L.G. (1993). Are we confusing self-esteem and narcissism? Young Children, 2-3.

Katz, L.G. (1994) Reflections on the Reggio Emilia Approach. Chicago: ERIC Clearing House On Early Childhood Education.

Katz, L.G. (1995). Talks with teachers of young children: A collection. New Jersey: Ablex. 
Katz, L.G. (1996). Child development knowledge and teacher preparation: Confronting assumptions. Early Childhood Research Quarterly, 11(2), 135-146.

Katz, L.G., \& Weir, M.K. (1969). Help for teachers in preschools: A proposal. ERIC Clearinghouse on Early Childhood Education, Urbana, III.

Keating, A. (Ed.). (2009). The Gloria Anzaldua reader: Latin American otherwise. Duke University Press.

Keating, A. (2016). EntreMundos/AmongWorlds: New perspectives on Gloria Anzaldua. Springer Publishing.

Kelly, D., \& Lukaart, S. (2005). The hive project: Building community and supporting environmental awareness through recycled materials. Innovations, 12(3), 14-18.

Kilbourn, B. (2006) The qualitative doctoral dissertation proposal. Teachers College Record, 108(4), pp.529-576.

Kinkaid, J. (1992). Child-loving: The erotic child in and Victorian literature. New York: Routledge.

Kissinger, K. (2017). Anti-bias education in the early childhood classroom: Hand in hand, step by step. Routledge.

Kohlberg, L. A. (1966). A cognitive-developmental interpretation of children's sex role concepts and attitudes. In E.E Maccoby (Ed.), The development of sex differences (pp. 82-173). Stanford University Press.

Kohn, A. (2005). Unconditional parenting: Moving from rewards and punishments to love and reason. Atria Books.

Konstantoni, K. (2011). Young children's perceptions and constructions of social identities and Social implications: promoting social justice in early childhood (Doctoral thesis, The University of Edinburgh, Scotland). Retrieved from https://www.era.lib.ed.ac.uk/handle/1842/5572

Konstantoni, K. (2012). Children's peer relationships and social identities: Exploring cases of young children's agency and complex interdependencies from the minority world. Children's Geographies, 10(3), 337-346.

Kuhn, T. S. (1962). The structure of scientific revolutions. University of Chicago Press.

Latour, B. (2004). The politics of nature. Harvard University Press. 
Lave, J. (1996). Teaching, as learning, in practice. Mind, Culture, and Activity, 3(3), 150164.

Lesnik-Oberstein, K. (1994). Children's literature: Criticism and the fictional child. Oxford: Clarendon Press.

Lewin, K. (1935). A dynamic theory of personality. McGraw Hill Book Company Inc.

Lewis, M. (1990). Social knowledge and social development. Merrill-Palmer Quarterly, 36, 93-116.

Letourneau, N., \& Allen, M. (1999). Post-positivistic critical multiplism: A beginning dialogue. Journal of Advanced Nursing, 30(3), 623-30.

Lichtman, M. (2013). Qualitative research in education: A user's guide. Sage Press.

Livesley, W., \& Bromley, D. (1973). Person perception in childhood and adolescence. London: Wiley.

Louv, R. (2008). Last child in the woods: Saving our children from nature deficit disorder. Algonquin Books of Chapel Hill.

Mackey, G. (2012). To know, to decide, to act: The young child's right to participate in action for the environment. Environmental Education Research, 18(4).

Malaguzzi, L. (2016). The final years: 1990-93. In M. Castagnetti, C. Giudici, C. Rinaldi, V. Vecchi, and P. Moss, Editors (Eds.), Loris Malaguzzi and the schools of Reggio Emilia : A selection of his writings and speeches, 19451993 (Contesting early childhood) (J McCall, Trans.). Abingdon, Oxon ; New York, NY: Routledge, Taylor \& Francis Group.

Malaguzzi, L. (1993). For an education based on relationships, Young Children, pp. 9-13.

Malaguzzi, L. (1996). The importance of seeing yourself again. In Catalogue of the exhibit The Hundred Languages of Children. Reggio Children.

Maple, M., \& Edwards, H. (2010). Locating and understanding voices in narrative inquiry. In V. Minichiello \& J. A. Kottler (Eds.), Qualitative Journeys: Student and Mentor Experiences with Research (pp. 33-48). CA: Sage.

Marcon, R. (1994). Doing the right thing for children: Linking research and policy reform in the District of Columbia public schools. Young Children, 50(1), 8-20. 
Maynes, M.J., Pierce, J., \& Laslett, B. (2008). Telling stories: The use of personal narrative in the social sciences and history. Cornell University Press.

Maxwell, J.A. (2005). Qualitative research design: An interactive approach. Ca: Sage.

Mayer, M. (2001). All by myself. Random House Books.

McKeown, R. (2000). Education for sustainability development toolkit. Waste Management Research and Education Institution.

McLaren, P. (2014). Life in schools. U.S: Routledge.

McLaren, P., \& Leonard, P. (1993). Paulo Freire: A critical encounter. Routledge.

Mead, G.H. (1934). Mind, self and society. C.W. Morris (Ed.). University of Chicago Press.

Montoya, D. (2009). Reclaiming resources: The art and learning in reusing materials. In Nothing Without Joy: The Delight of Learning Together (pp.9-10.).UNM Family Development Program.

Mooney, C.G. (2013). Theories of childhood: An introduction to Dewey, Montessori, Erikson, Piaget \& Vygotsky. Redleaf Press.

Morgan, D. (1997). Focus groups as qualitative research. Sage Publications.

Morgan, D. (2016). Essentials of dyadic interviewing. Routledge.

Moss, P. (2016). Why can't we get beyond quality? Contemporary Issues in Early Childhood Journal. ISSN: 1463-9491.

NAEYC. (2009). On responding to cultural and linguistic diversity. National Association for the education of Young Children. Retrieved from: http://www.naeyc.org/files/naeyc/file/positions/diversity.pdf

Newman, L.S., \& Ruble, D.N. (1988). Stability and change in self-understanding: The early elementary school years. Early Child Development and Care, 40, 77-99.

Odegard, N. (2012). When matter comes to matter-working pedagogically with junk materials. Education Inquiry, 3(3), 387-400. 
Orozco-Mendoza, E. (2008). Borderlands theory: Producing border epistemologies with Gloria Anzaldua. (Masters thesis, Virginia Tech). Retrieved from: http://hdl.handle.net/10919/32268

Orr, D. (1992). Ecological literacy: Education and the transition to a postmodern world. SUNY Press.

Padilla, R.V., \& Martinez, R.O. (2005). Personal stories, voice and presence in academe: A dialogic response to Aguirre. International Journal of Qualitative Studies in Education, 18(2), 199.

Padilla, R. V., \& Montiel, M. (1998). Debatable diversity: Critical dialogues on change in American universities. Maryland: Rowman \& Littlefield.

Palahniuk, Chuck. (2004). Diary. Anchor Publishing.

Palaiologou, I. (2011). Child observation in the early years. Sage Publications.

Parnell, W. Downs, C., \& Cullen, J. (2017). Fostering intelligent moderation in the next generation: insights from Remida-inspired reuse materials education. The New Educator, 1-17. doi:10.1080/1547688x.2017.1331094

Parnell, W. (2010). Image of the teacher in educational leadership. International Journal of Educational Leadership Preparation, 5(3).

Patton, M.Q. (2002). Qualitative research methodology. Sage Publications.

Pavlou, V. (2009). Understanding young children's three-dimensional creative potential in art making. International Journal of Art and Design Education, 28(2), 139-150.

Peck, J.T., McCraig, G., \& Sapp, M.E. (1988). Kindergarten policies: What is best for children? Washington D.C, National Association for the Education of Young Children.

Pelo, A. (2006). At the crossroads: pedagogical documentation and social justice. In A. Fleet, C. Patterson, J. Robertson (Eds.), Insights: Behind Early Childhood Pedagogical Documentation. Castle Hill: Pademelon Press.

Pena, D.G. (1998). Chicano culture, ecology, politics: Subversive kin. University of Arizona Press.

Pena, D.G. (2005). Mexican Americans and the environment: Tierra y vida. The University of Arizona Press: Tuscon. 
Phillips, D.C. (1995). The good, the bad, and the ugly: The many faces of constructivism. Educational Researcher, 24(7), 5-12.

Piaget, J. (1945). Play, Dreams and imitation in childhood. New York: Norton.

Piaget, J. (1952). The origins of intelligence in children. International Universities Press.

Piaget, J. (1958). The growth of logical thinking from childhood to adolescence. $A M C$, 10(12).

Piaget, J. (1970). Piaget's theory. In P. Mussen (Ed.), Carmichael's Manual of Child Psychology. New York: Wiley.

Pinkola Estes, C. (1992). Women who run with the wolves: Myths and stories of the wild womanarchetype. Ballantine Books.

Pinnegar, S. (2006). Afterword: Re-narrating and indwelling. In D.J. Clandinin et, al. Composing Diverse Identities: Narrative Inquiries into The Interwoven Lives of Children and Teachers (pp. 176-190). New York: Routledge.

Pitts, AJ. (2016). Gloria Anzaldua's autohistoria-teoria as an epistemology of selfknowledge/ignorance. Retrieved from https://doi.org/10.1111/hypa.12235

Pizarro, M. (2004). Searching for curanderas: A quest to revive chicana/o studies. Journal of and Education, 3, 145-164.

Poniatowska, E. (1969). Here’s to you, Jesusa! Penguin Books.

Popper, K. (1945) The open society and its enemies. Routledge.

Pritchard, E.D. (Ed.). (2017). Sartorial politics, intersectionality, and queer worldmaking. QED: A Journal of GLBTQ Worldmaking, 4(3).

Project Zero and Reggio Children, Italy. (2001). Making learning visible: Children as individual and group learners. Reggio Emilia, Italy: Reggio Children S.r.l. and Reggio Children/USA.

Prout, A., (2011). Taking a step away from modernerity: Reconsidering the new sociology of childhood. Global Studies of Childhood,1(1), 4-14.

Pruett, K.D. (1999). Me, myself, and I: How children build their sense of self-18 to 36 months. New York: Goddard. 
Quintana, S. M. (1998). Children's developmental understanding of ethnicity and race. Applied-and-Preventive-Psychology, 7, 27-45.

Raag,T., \& Rackliff, C.L. (1998). Preschoolers' awareness of social expectations of gender: Relationships to toy choices. Sex Roles, 38(9), 685-700.

Raburu, P. (2015). The self-Who am I? Children's identity and development through early childhood education. Journal of Educational and Social Research, 5(1), 95.

Rathje, W., \& Murphy, C. (2001). Rubbish! The archaeology of garbage. Universityof Arizona Press.

Rau, C., \& Ritchie, J. (2014). Ki te whai ao, ki te ao marama: Early childhood understandings in pursuit of social, cultural, and ecological Justice. In M. N. Bloch, B. B. Swadener \& G. S. Cannella (Eds.), Reconceptualizing Early Childhood Care and Education. Critical Questions, New Imaginaries and Social Activism: A Reader (pp. 109-130). New York: Peter Lang.

Rebolledo, T. D. (2006). Prietita y el otro lado: Gloria Anzaldúa's literature for children. PMLA, 121(1), 279-784.

Reggio Children. (2004). Children art, artists: The expressive languages of children, the artistic Language of Alberto Burri. Reggio Children Publisher.

Reggio Children. (2005). REMIDA day muta...menti (English Version). Reggio Children Publisher.

Reggio Children. (1998/2011). Children, space, relations: Metaproject for an environment for young children. Reggio Children Publisher.

Reggio Children. (2016). Bikes .....lots! Reggio Children Publisher.

Reggio Children. (2019). Remida. Retrieved from http://www.reggiochildren.it

Reggio Children (Eds.). (1996). Catalogue of the exhibit the hundred languages of children. Reggio Emilia, Italy: Reggio Children.

Reggio Children S.R.I. (1996). I cento linguagui dei bambini/The hundred languages of children: Narrative of the possible. Catalogue of the Exhibit. USA: Reggio Children.

Reinisch, S., \& Parnell, W. (2006). The hundred is there! The role of physical spaces, aesthetic qualities, and documentation in a vibrant learning community. Design 
Share: Designing for the Future of Learning Online Journal. Retrieved from www.designshare.com/index.php/articles/hundred-1.

Rinaldi, C. (1998). The spaces of childhood. In G. Ceppi \& M. Zini (Eds), Children, spaces, Relations: Metaproject for an Environment for Young Children (pp. 114120). Reggio Emilia, Italy: Reggio Children.

Rinaldi, C. (2006). In dialogue with Reggio Emilia: Listening, researching and learning. New York: Routledge.

Ritchie, J., Craw, J., Rau, C., \& Duhn, I. (2013). Ko koe ki tena, ko ahau ki tenei kiwai o te kete: Exploring collaboration across a range of recent early childhood studies. In J. Duncan, \& L. Connor, (Eds.). Research Partnerships in Early Childhood Education: Teachers and Researchers in Collaboration (pp.93-113). New York: Palgrave Macmilan.

Rochat, P. (2001). Origins of self-concept. In G. Bremner \& A. Fogel (Eds.), Blackwell Handbook of Infant Development (pp. 191-212). Oxford: Blackwell.

Rodriguez, J. (1994). Our Lady of Guadalupe: Faith and empowerment among MexicanAmerican women. Austin: University of Texas Press.

Rubin, H., \& Rubin, I. (1995). Qualitative interviewing: The art of hearing data.Ca: Sage.

Schweinhart, L. J., Weikart, D. P., \& Larner, M. B. (1986). Consequences of the three preschool curriculum models through age 15. Early Childhood Research Quarterly, 1, 15-45.

Sfard, A. (1998). On two metaphors for learning and the danger of choosing just one. Educational Researcher, 27 (2), 4-13.

Sfard, A., \& Prusak, A. (2005). Telling identities: In search of an analytic tool for investigating learning as a culturally shaped activity. Educational Researcher, 34(4), 14-22.

Shapiro, B. (1994). What children bring to light: A constructivist perspective on children's learning in science. New York: Teachers College Press.

Sharova, E. (2012). The Nicolas Bourriaud approach applied to Remida: Center for creative recycling within the discourses of contemporary aesthetics and pedagogy. (Master's thesis). Available from academia.edu Dissertations and Theses database. 
Skattebol J. (2006). Playing boys: The body, identity and belonging in the early years. Gender and Education,18(5), 507 522. doi.org/10.1080/09540250600881667.

Solis Ybarra, P. (2009). Borderlands as bioregion: Jovita González, Gloria Anzaldúa, and the twentieth-century ecological revolution in the Rio Grande Valley. MELUS, 34(2), 175-189.

Stipek, D., Feiler, R., Daniels, D., \& Milburn, S. (1995). Effects of differential instructional approaches on young children's achievement and motivation. Child Development, 66(1), 209-223.

Stroot, S., Keil, V., Stedman, P., Lohr, L., Faust, R., Schincariol-Randall, L., Sullivan, A., Czerniak, G., Kucinski, J., Orel, N., \& Richter, M. (1998). Developmental stages of Teachers. Peer assistance and review guidebook. Columbus, $\mathrm{OH}$ : Ohio Department of Education.

Swanson, D.P., Cunningham, M., Youngblood, J., \& Spencer, M.B. (2009). Racial identity development during childhood. In H.A. Neville, B.M. Tynes \& S.O. Utsey (Eds.), Handbook of African American Psychology (pp. 269-281). Thousand Oaks: Sage Publications.

Tajfel, H. (1974). Social identity and intergroup behaviour. Social Science Information, $13,65-93$.

Tajfel, H. \& Turner, J. C. (1986). The social identity theory of inter-group behavior. In S. Worchel \& W. G. Austin (Eds.), Psychology of Intergroup Relations. Chicago: Nelson-Hall.

Tarr, P. (2001). Aesthetic codes in early childhood classrooms: What art educators can learn from Reggio Emilia. Retrieved from http://www.designshare.com/Research

Tarr, P. (2003). Reflections on the image of the child: Reproducer or creator of culture. Art Education, 56(4), 6-11.

Tarr, P. (2008). New visions: Art for early childhood: Essential for early learning. Art Education, 61(4), 19-24.

Taylor, A. \& Pacini-Ketchabaw, V. (2015). Learning with children, ants, and worms in the Anthropocene: towards a common world pedagogy of multispecies vulnerability. Pedagogy, Culture \& Society, 23(4), 507-529. 
Thoits, P.A., \& Virshup, L.K. (1997). Me's and we's: forms and functions of social identities. In R.D Ashmore \& L.J. Jussim (Eds.), Self and Identity: Fundamental Issues. Rutgers series on Self and Social Identity (pp. 106-133). New York: Oxford University Press.

Thomas, S. (1987). The last navigator: A young man, an ancient mariner, the secrets of the sea. Book Surge Publishing.

Thomashow, M. (1995). Ecological identity: Becoming a reflective environmentalist. Massachusetts Institute of Technology.

Topal, K., Gandini, L. (1999). Beautiful Stuff! Learning with found objects. Davis Publications Inc.

Trahar, S. (Ed.). (2013). Contextualising narrative inquiry: Developing methodological Approaches for local contexts. Routledge.

Triandis, H. C. (1995). Collectivism versus individualism: A reconceptualization of a basic concept in cross-cultural social psychology. In C. Bagley \& G. K. Verma (Eds.), Personality, cognition and values: Cross-cultural perspectives of childhood and adolescence. London: Macmillan.

UNCED. (1992). Agenda 21: Programme of action for sustainable development. Rio declaration on environment and development. New York: United Nations.

UNESCO. (2008). The contribution of early childhood education to a sustainable society. http://unesdoc.unesco.org/images/0015/001593/159399e.pdf

UNICEF. (2001). Article 29 Convention on the Rights of the Child. Retrieved from http://www.unicef-irc.org

U.S. Environmental Protection Agency, (2013). Municipal solid waste in the United States: 2013 facts and figures. Retrieved from: http://www.epa.gov/solidwaste/nonhaz/municipal/

van Mannen, M. (1990). Researching lived experience: Human science for an action sensitive pedagogy. SUNY Press.

Vecchi, V. (2010). Art and creativity in Reggio Emilia: Exploring the role and potential of ateliers in early childhood education. New York: Routledge.

Vila, P. (Ed.). (2003). Ethnography at the border. University of Minnesota Press. 
Von Glasersfeld, E. (1984). An introduction to radical constructivism. In P. Watzlawick (Ed.), the invented reality (pp. 17-40). New York: W.W Norton.

Vygotsky, L. S. (1962). Thought and language. Cambridge MA: MIT Press.

Vygotsky, L. (1978). Interaction between learning and development. In M. Cole, V. John-Steiner, S. Scribner., \& E. Souberman (Eds.). Mind in Society: The Development of

Higher Psychological Processes (pp. 70-91). Harvard University Press.

Vygotsky, L. S. (1979). Consciousness as a problem in the psychology of behavior. Soviet Psychology, 17, 3-35.

Wells, K. (2011). Narrative Inquiry. Oxford University Press.

Weiss, H., Caspe, M., \& Lopez, E. (2006). Family involvement in early childhood education. Harvard Family Research Project, 1, 1-8.

Weiss, H., \& Lopez, E. (2015). Engage families for anywhere, anytime learning. Phi Delta Kappan, 96(7), 14-19.

Wexler, A. (2004). A theory for living: Walking with Reggio, Art Education, 57(6), pp. 13-17.

Whitney, T. (1999). Kids like us: Using persona dolls in the classroom. Redleaf Press.

Wien, C.A., Gyuvesky, V., \& Berdoussis, N. (2011). Learning to document in Reggioinspired education. Early Childhood Research and Practice. 13(2).

Wilson, R. (2012). Nature and young children: Encouraging creative play and learning in natural environments. New York: Routledge.

Woodhead, M. (2006). Changing perspectives on early childhood: theory, research and policy. International Journal of Equity and Innovation in Early Childhood, 4(2), $1-43$.

Zero to Three. (2004). Infant and early childhood mental health: Promoting healthy social and emotional development. Washington DC: National Center for Infants, Toddlers and Families. 


\section{APPENDIX A \\ RECRUITMENT MATERIALS}

Email Recruitment:

Do you have experiences with Reggio-inspired creative reuse with children ages 2-5 that you would like to share? My name is Michelle Domingues. I am a doctoral candidate at Portland State University and the coordinator of Inventing Remida Portland Project. My inquiry focuses on Reggio-inspired early childhood educator's experiences working with reuse materials with children (from one to five years of age for a minimum of 3 years.) In fall 2015 and winter of 2016, you are invited to participate in a research study investigating early childhood educator's experiences with Remida-inspired reuse materials. The purpose of this narrative study is to capture master early childhood educator's experiences in their own professional journey and explore how engagement with identity studies can intersect with reuse practice. The study includes a preliminary phone interview and three 120 minute dyadic and focus group interview sessions. During the interviews you will have an opportunity to create a reuse representation of identity and to share documentation of a reuse project. If you are interested in learning more about the study, please contact Michelle Domingues at:

$503-432-8710$

Aldama@pdx.edu 


\section{APPENDIX B \\ INITIAL PHONE SCREENING}

Interviewee: Interview \#:

Time: Date: Location:

Thank you for calling to find out more about my research study. This call should take approximately 15 minutes. I am a doctoral study at Portland State University in the Graduate School of Education. The purpose of this research study is seeking to understand how Reggio-inspired early childhood educator's experience working with reuse materials with children from one-to-five years of age for a minimum of 3 years. The study involves three 120 minute interviews; two in dyads and one in a focus group of four Reggio-inspired educators. Using reuse collage, you will reflect on your professional experiences in working in early childhood education and with identity. As part of the dissertation study, I am asking individuals interested in participating in the study to identify some basic personal and professional demographic information. Do you think you would be interested in in participating in this study?

\{f No:\} Thank you very much for calling.

\{If Yes:\} Before enrolling individuals in this study, I need to determine if you are eligible. I would now like to ask you a series of questions about your personal and professional demographical information. There is a possibility that some of these questions might make you uncomfortable. If so, please let me know. You don't have to answer any of those questions if you do not want to. You also need to understand that all 
information I receive from you by phone, including your name and any other identifying information, will be strictly confidential and will be kept locked in a file cabinet on the PSU campus. The purpose of these questions is only to determine if you are eligible for the larger study. Remember your participation is voluntary; you do not have to complete these questions. Do I have your permission to ask you these questions?

\{If No:\} Thank you very much for calling.

\{If Yes:\} I have several of personal demographic questions to ask you. - Are you at least 21 years of age? - Do you live in the Portland Metro area? Thank you. Additionally, I have a series of five professional questions to ask you. - Are you currently working in a Reggio Emilia inspired program? • Are you currently working with children, ages 1-5 years of age? $\bullet$ How long have you worked with this age group? $\bullet$ Are you familiar with Remida Cultural Centers? • Do you work with reuse material in the classroom?

\{If No to one or more questions:\} Thank you very much for calling. I sincerely appreciate the time you took to talk with me today.

\{If Yes:\} Thank you for answering the questions. You are eligible to participate in the interview study in exploring Reggio inspired early childhood educator's experiences working with reuse materials with children from one-to-five years of age. This interview is designed in two-parts. Through the use of reuse collage, you will reflect on your professional experiences in working in early childhood reuse and identity education, and then you will share your story through pedagogical documentation of your work with creative reuse. For the purpose of this study, a collage is defined as an art medium that 
uses various forms materials and objects pasted over a surface. Collage is often used to help people tell their stories. The purpose of a collage in this research project is to support you in telling your story of your experiences in your professional journey with social identity. There are no expectations about the 'look' of the collage. It is a tool for telling your story. In this research study, the collage materials will use different types of reuse materials to represent your thinking. You are welcome and encouraged to bring any documentation: pictures, photos, quotes or other materials that help you to tell your story of your work with reuse and identity as an early childhood educator. The interview format will include an introduction and a review of the Informed Consent document, telling you about the study and laying out guidelines of what to expect. Then photos will be taken of any materials you choose to bring to the interview. A tour of the room and the tools available will be next, followed by time to work on your collage in pairs. Next a dyadic interview will take place, where we will discuss your identity as an early childhood educator and social constructivist, using the collage during the first interview and documentation during the second interview as support. The last part of the interview will be to take photos of your collage. Are you interested in participant in the study?

\{If No:\} Thank you very much for calling. I sincerely appreciate the time you took to talk with me today. Would you like my phone number or Inventing Remida Portland website address?

\{If Yes:\} The study is conducted during the months December 2015 through February 2016 at a local creative reuse studio in the Portland metro area. Thank you for agreeing to 
a location. What times and dates work best for you for an interview? What would be the best way to contact you to confirm the times and dates of your interviews? Thank you for agreeing to participate in this study. I will contact you within 48-hours with the times and locations of you interview. My contact information is:

Michelle Domingues 503-432-8710

aldama@pdx.edu 
APPENDIX C

\section{RESEARCH LOG}

\begin{tabular}{|l|l|l|}
\hline Date & Activity & Material/Notes \\
\hline & & \\
\hline & & \\
\hline
\end{tabular}


APPENDIX D

\section{DATA COLLECTION AND TIMELINE PROCEDURES}

$\underline{\text { Dates of Project }}$

September 2015 to October 2015

September 2015 to October 2015

September 2015 to November 2015

screening script

September 2015 to November 2015

Response

November 2015 to January 2016

using interview scripts and email prompts

January 2016 to April 2016

Artifacts Analytic Memos

April 2016 to August 2016

\section{$\underline{\text { Activity Data Collected }}$}

Participant Recruitment

Activities entered into Research Log

Initial Phone Screening using phone

Email confirmation of interview

Dyadic and Focus Group Interviews

Audio recording Transcripts

Interpretation of Data 


\section{APPENDIX E \\ INTERVIEW PROTOCOL}

Interviewee:

Interview \#:

Time:

Date:

Location:

Introduction to Interview and Collage

My name is Michelle Domingues and I am a doctoral student at Portland State University in the Graduate School of Education and Graduate Assistant at Helen Gordon

Development Center. It is good to meet you in person. Here is my contact information if you need to get ahold of me during the process. My contact information is Michelle Domingues aldama@pdx.edu or you can call me at 503-432-8710.

Thank you for your interest participating in the dyadic and focus group interview portion of the research study. We will spend 120 minutes together. The purpose of this research study is seeking to explore Reggio-inspired early childhood educators' experiences in reuse and identity studies with young children. This interview is designed in two-parts. Through the use of collage, you will reflect on your professional experiences in working with identity studies in early childhood education and then you will share your experiences in creative reuse education. I would like to start our time together by reviewing the Informed Consent Document. An informed consent document provides you with information as to what will happen during the interviews and will provide information about what you can expect during our time together, and speak to the collection and use of data. I brought a copy for you to keep for your records today. At any 
point that you feel uncomfortable, then please feel free to let me know. If you wish to leave at any time, please feel free to do so. What questions do you have? Let us review the informed consent form. Review Form. Do you have any questions?

Thank you for participating in this study.

Introduction to reuse Collage as Part of Interview

Collage is often used to help people represent their thinking. The purpose of this collage is to invite you to make an impermanent representation of each other with reuse materials. There are no expectations about the "look" of the collage. As part of the interview process, we will be working on a collage to facilitate some of your thoughts about your professional journey with social identity and reuse. A professional journey can be defined in many ways... how you came to work in early childhood, the experiences that you have had along the way, a memorable moment... and can describe your professional journey. Let's look at the materials. For the base of your collage, I have several pieces of felt ovals in different shades of skin tone that you can use for the base. For the collage, you can use any of material provided. There are natural and found objects, manufacturer discards and scissors. So, in collage, you can look at materials and think about what materials would help you in making a symbolic and or literal representation of your partner. One advantage of collage is that you can move objects around. So, all of these materials you use can come together, and you can organize them in a way you find represents your pair. On the table, I have left a piece of paper with the prompts about identity and reuse. . Once you are ready to start, I will leave the room. 
The digital recorders will capture your conversation in my absence. Please take as much time as you wish. I will be outside the room if you have any question. When you are ready, please come get me to begin the interview. I will now leave you. Please take your time. When you are ready, I will be outside the door. Participants works on reuse collage. When resuming the interview: Thank you, would you like to take a break? When you are ready, we can start on the second part of the interview. I have a digital recorder set-up to capture our conversation now as well. Also, during the interview, you might see me take notes from time-to-time, which is just to help me remember when I look at our interview later. When we are done with the interview, I would like to take photos of your reuse collages.

Dyadic Interview \#1

Ice breaker: What was it like to represent yourself with reuse materials?

1. Please share with one another the story of how you came to identify as a social constructivist and Reggio- inspired early childhood educator?

2. How did you come to be Remida-inspired?

3. What does identity studies in early childhood education mean to you and how have you approached identity studies with young children as a social constructivist educator?

4. How did visiting Reggio Emilia Remida impress you? 
Before I take photographs of your collage, is there anything else you would like to share? I want to express my appreciation for your participation in this study. Would you like me to send you photos of your collage, your interview and, or a copy of the completed study? You have my contact information and, if I have any questions, may I follow-up with you?

Materials List

Collage Base • Oval felt material • Regular and crafty scissors Natural materials, manufacturer discards, found objects.

Dyadic Interview \#2

Ice breaker: What was it like to represent your pair?

1. What does documentation mean to you? Please share your documentation of reuse and identity studies.

2. Can you share stories with one other about your experiences with reuse and children and what is it like to work with a co-teacher, and families when you co-construct social identities with your students using reuse materials?

3. What is difficult, challenging or rewarding about working with reuse materials with children and how does that affect you?

4. Do you identify as an atelierista/what is your image of the teacher?

Focus Group Interview \#1 
For my focus interview design, I chose to pose the prompt: Create a Remida inspired persona doll using reuse materials and make a story about the doll by choosing to answer the following questions about the doll and three broadly stated questions:

1. How did the doll come to identify as a social constructivist and Reggio inspired early childhood educator?

2. What does identity studies in early childhood education mean to the doll? How has the doll approached identity studies with young children as a social constructivist?

3. Will you share about the doll's visits to Remida in Italy and the impression that it had on the doll?

Wrap up: Does anyone want to share anything about this process? Or what you foresee as the relationship between the persona dolls you have created? A future collaboration between them? 


\section{APPENDIX F}

\section{INFORMED CONSENT}

Study title: Identifying with Remida: Early Childhood Educators' Experiences with Reuse Materials in Reggio inspired Identity Studies.

Principle investigator: Co-Investigator: Will Parnell and Michelle Domingues Graduate School of Education Graduate School of Education Curriculum and Instruction Curriculum and Instruction PO Box 751527 SW Hall Street, Suite 300 Portland, OR 97207-0751 Portland, OR 97201 503-725-3091 503-866-0454 parnellw@pdx.edu , aldama@pdx.edu.

Introduction to the study: You are being invited to take place in a research study. I am a doctoral study at Portland State University in the Graduate School of Education. The purpose of this research study is seeking to explore Reggio-inspired early childhood educator's experiences with reuse and social identity. The study involves three 120 minute dyadic and focus group interviews. In the three interviews and two email prompts you will have a chance to reflect on professional experiences in working with creative reuse and identity in early childhood education. It is important that you understand why the research is being done and what it will involve. Please take the time to read the following information carefully. Please ask me if there is anything that is not clear or if you need more information. What will happen during the study: The study will require you to agree to an initial phone interview. The initials phone interview will be approximately 15 minutes. You will participate in three interviews ranging from 90-120 minutes. The study is conducted during the months September 2015 through January 
2016 at a location in a local creative reuse studio in the Portland metro area. The interviews have two-parts, a self-reflective process using collage to think about the research question as it relates to your professional life and an interview about your experiences, using pedagogical documentation as a reference. Data will be gathered from your collage and interviews. The data collected includes an audio recording of our conversation, photos of your collages, interview notes, and email prompted research reflections following the interviews and from the collage and interviews. The audio will be used to transcribe the conversation and check for concepts and themes from the interview. The collage is not yours to take at the end of the interview as it will be impermanent. A copy of your interview transcription, photos of your collage, and final dissertation will be available on request. Risks: The risk of this study is minimal. These risks are similar to those you experience when you share work-related information with others. If the topics challenge you beyond your comfort level, you may choose to decline to answer any questions in the study and you may terminate your involvement at any time if you choose. Benefits: There will be no direct benefit to you from this study beyond the pleasure of sharing your experiences. However, I hope that the information from this study will bring early childhood educators' voice to the field of early childhood education for sustainability. How is participant's (your) privacy protected: For the purpose of this research project, your interview will be confidential unless you give permission for the use of your identity. Every effort will be made by the researcher to preserve your confidentiality including the following: Assigning code names and numbers to all participating individuals that will be used on all researcher notes and documents: $\bullet$ Notes, 
audio, transcriptions, transcribed notes, and any other identifying participant information will be kept in a locked file cabinet in the personal possession of the researcher. When no longer necessary, all materials will be destroyed. Collages will be returned to you if desired. - Information from this research study will be used solely for the purpose of this study and any publications that may result from this study. Any final publications will remain confidential unless you have agreed to waive confidentiality. • You have the opportunity to obtain a transcribed copy of their interview and photos of their collage. Please tell the researcher if a copy of the interview and photos of the collage are desired. You should be aware that the researcher is a mandated reporter by Oregon law and confidentiality is not protected if the researcher is legally required to make a report on an incident involving a child. Contact: Should you have any questions about the research or any related matters, please contact co-investigator Michelle Domingues at aldama@pdx.edu or at 503-866-0454. If you have any questions about the coinvestigator, please contact principle investigator, Will Parnell at 503-725-3091 or parnellw@pdx.edu. Institutional Review Board: If you have any questions regarding your rights as a research subject, or if problem arise which you do not feel like you can discuss with the principle investigator, please contact the Institutional Review Board at hsrrc@pdx.edu. Voluntary Participation: Your participation in this study is voluntary. It is up to you to decide whether or not to take part in this study. If you decide to take part in this study, you will be asked to sign a consent form. If you decide to take part in this study, you are still free to withdraw at any time and without giving a reason. You are free to answer or not answer any question or questions if you choose. Unforeseen risk: There 
may be risks that are not anticipated. However, every effort will be made to minimize any risks. Cost to participate: There is no cost for your participation in this study.

Compensation: There is no compensation for this study. PLEASE READ THE FOLLOWING STATEMENT AND SIGN BELOW IF YOU AGREE.

I have had the chance to ask any questions I have about this study and my questions have been answered. I have read the information in this consent form and I agree to participate.

Select One:

o I give permission for my real identity to be used in the research (please initials)

o I wish for my identity to remain confidential (please initial)

Signature of the participant

Date 
APPENDIX G

\section{ARTIFACT CATALOGUE}

Artifact Number: Date Received:

Artifact Type: $\square$ Photo $\square$ Personal representation $\square$ Other : Notes:

Location:

Description of Artifact:

Event or Contact associated with artifact:

Presentation of artifact: $\square$ Personal representation for collage $\square$ Other:

Brief Summary of artifact:

Printed name of the participant 